COMPUTER-PROGRAM DOCUMENTATION OF AN INTERACTIVE-ACCOUNT ING

MODEL TO SIMULATE STREAMFLOW, WATER QUALITY, AND

WATER-SUPPLY OPERATIONS IN A RIVER BASIN

By Alan W. Burns

U.S. GEOLOGICAL SURVEY

Water-Resources Investigations Report 88-4012

Prepared in cooperation with the

SOUTHEASTERN COLORADO WATER CONSERVANCY DISTRICT

Denver, Colorado

1988 


\section{FIGURES}

Figure 1. Map showing sample basin with nodes and incremental areas---

Page

2. Organizational chart of the subroutines used for streamflow and water-quality simulation--- 8

3-11. Examples of various information as viewed on a computer terminal:

3. A schematic for node location in the sample basin------

4. A schematic for annual average streamflow in the sample basin-.-... 13

5. A schematic for monthly streamflow in the sample basin-- 15

6. A hydrograph of streamflow for a node in the sample basin-.-...-.

7. A table of monthly streamflow for a node in the sample basin--.-. 19

8. A statistical summary for a node in the sample basin---- 20

9. A comparison histogram for a node in the sample basin--- 21

10. Two frequency curves for a node in the sample basin----- 23

11. A residual histogram for a node in the sample basin----- 25

12. Map showing sample basin with diversion ditches and pipelines 27

13. Graph showing unit-response functions for five selected stream-depletion factors (SDF) 30

14. Organizational chart of all model subroutines---- 31

15-20. Examples of various information as viewed on a computer terminal:

15. A schematic for reservoir location in the sample basin--

16. A schematic for water-user location in the sample basin-

17. A schematic for final ground-water storage in the sample basin- 39

18. A schematic for period-of-record average water use in the sample basin-- 41

19. A statistical summary for a water user in the sample basin--.- 43

20. A statistical summary for a reservoir in the sample basin--.-. 
COMPUTER-PROGRAM DOCUMENTATION OF AN INTERACTIVE-ACCOUNTING

MODEL TO SIMULATE STREAMFLOW, WATER QUALITY, AND

WATER-SUPPLY OPERATIONS IN A RIVER BASIN

By Alan w. Burns

U.S. GEOLOGICAL SURVEY

Water-Resources Investigations Report 88-4012

Prepared in cooperation with the

SOUTHEASTERN COLORADO WATER CONSERVANCY DISTRICT

Denver, Colorado 1988 
DEPARTMENT OF THE INTERIOR

DONALD PAUL HODEL, Secretary

U.S. GEOLOGICAL SURVEY

Dallas L. Peck, Director

For additional information write to:

\section{District Chief}

U.S. Geological Survey

Water Resources Division

Box 25046, Mail Stop 415

Federal Center

Denver, CO 80225-0046
Copies of this report can be purchased from:

U.S. Geological Survey Books and Open-File Reports Federal Center, Bldg. 810 Box 25425

Denver, CO 80225-0425 


\section{CONTENTS}

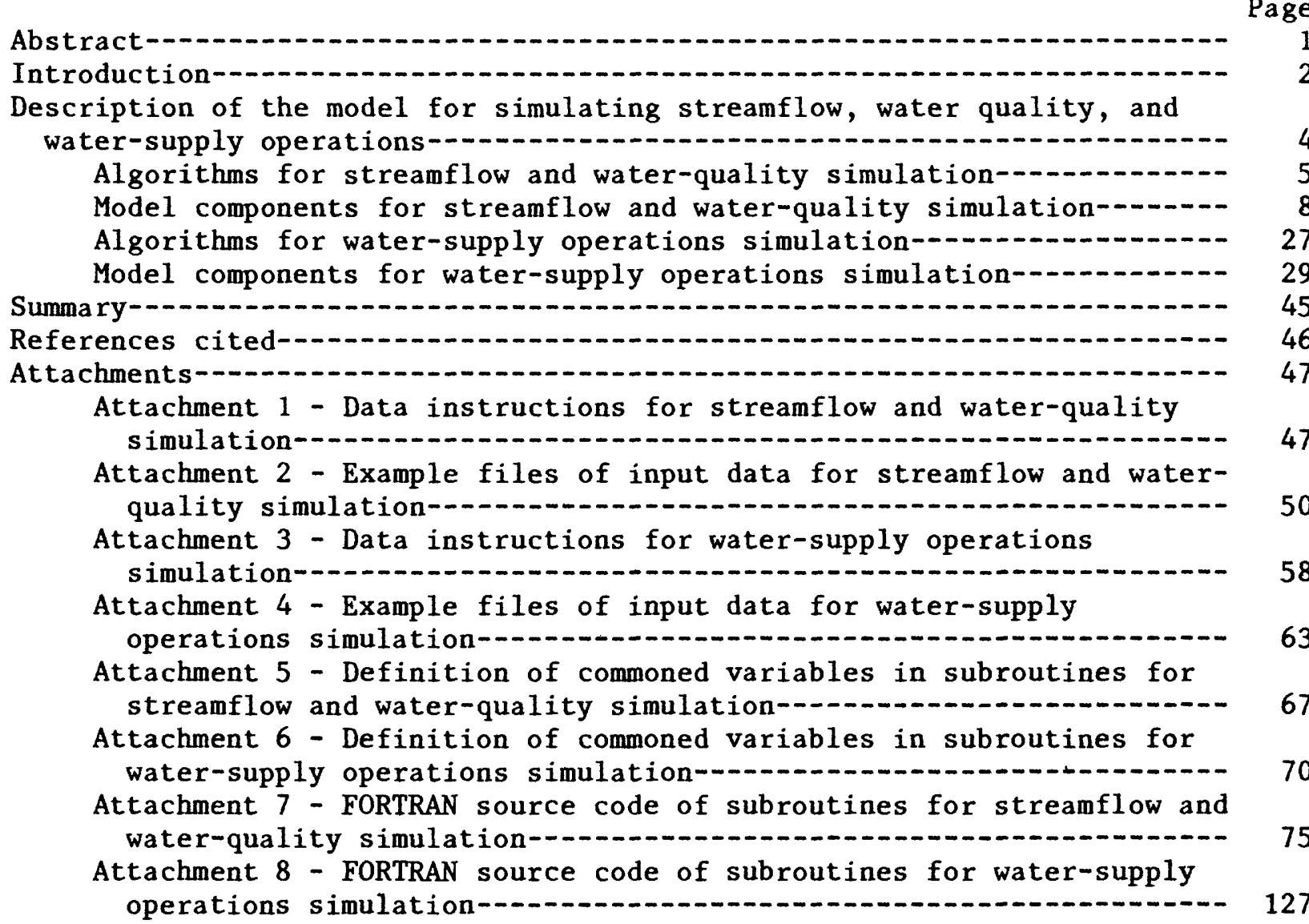




\section{FIGURES}

Page

Figure 1. Map showing sample basin with nodes and incremental areas----

2. Organizational chart of the subroutines used for streamflow and water-quality simulation-1 8

3-11. Examples of various information as viewed on a computer terminal:

3. A schematic for node location in the sample basin-.-.-

4. A schematic for annual average streamflow in the sample basin-... 13

5. A schematic for monthly streamflow in the sample basin-- 15

6. A hydrograph of streamflow for a node in the sample basin--... 17

7. A table of monthly streamflow for a node in the sample basin-1... 19

8. A statistical summary for a node in the sample basin---- 20

9. A comparison histogram for a node in the sample basin--- 21

10. Two frequency curves for a node in the sample basin--.-- 23

11. A residual histogram for a node in the sample basin----- 25

12. Map showing sample basin with diversion ditches and pipelines 27

13. Graph showing unit-response functions for five selected stream-depletion factors (SDF) 30

14. Organizational chart of all model subroutines-a. 31

15-20. Examples of various information as viewed on a computer terminal:

15. A schematic for reservoir location in the sample basin-- 33

16. A schematic for water-user location in the sample basin- 35

17. A schematic for final ground-water storage in the sample basin- 39

18. A schematic for period-of-record average water use in the sample basin- 41

19. A statistical summary for a water user in the sample basin- 43

20. A statistical summary for a reservoir in the sample basin-- 


\title{
COMPUTER-PROGRAM DOCUMENTATION OF AN INTERACTIVE-ACCOUNTING MODEL TO SIMULATE STREAMFLOW, WATER QUALITY, AND WATER-SUPPLY OPERATIONS IN A RIVER BASIN
}

By Alan W. Burns

\begin{abstract}
This report describes an interactive-accounting model used to simulate streamflow, water quality, and water-supply operations in a river basin. The model uses regression equations to compute flow from incremental (internode) drainage areas by using a time series of independent variables, such as snowpack, precipitation, or gaged flow. Water-quality concentrations and loads (typically dissolved solids) then are computed from regression equations by using streamflow as the independent variable. Streamflow and water-quality loads then are routed downstream--that is, they are accumulated downstream because there is no hydraulic routing in the model with the monthly time step.

The model is designed to operate interactively so that queries can be made during simulation, allowing considerable flexibility in output. Although most data are entered from pre-existing files, the data can be modified interactively to produce new management alternatives for simulation. Because of the interactive capability of the model, a major feature of the model is its color-graphic-output options.

The model also can simulate the water-supply operations and the simplified ground-water systems of a basin. Types of water users that can be simulated include agricultural, municipal, industrial, and in-stream users, and reservoir operators. Each water user has a list of potential sources, including direct diversions, ground-water pumpage, imports, or reservoir releases; this list is entered in the order that the sources will be used to meet demand. All direct diversions are controlled to conform to the basinwide, prior-appropriation, water-law priorities.

Documentation of the model includes a brief description of the model, simple organizational charts of the subroutines, and examples of the output graphics and tables. Detailed format instructions of the input data, examples of input data, definitions of program variables, and listings of the FORTRAN source code are provided as attachments at the back of the report.
\end{abstract}




\section{INTRODUCTION}

Most of the irrigated river basins in the semiarid western United States have similar hydrologic regimes. Snowmelt from adjacent mountains or uplands is the major source of flow in a river, which functions as a conduit to transmit water to lower elevations for irrigation and other uses. Because of the general scarcity of water, a set of water laws (based on the doctrine of prior appropriation) developed in the western United States that is distinct from the riparian system in the rest of the country. The prior-appropriation laws allocate and prioritize water to those users who first used the water. As development increased, the natural streamflow regime of these western river basins was transformed by return flows from irrigation, ground-water pumpage, storage in natural lakes and constructed reservoirs, and imports of water from other basins. The multiple reuse of the water as it is diverted, applied, returned, and rediverted, frequently concentrates the dissolved-solids contents of the water, leading to water-quality concerns and issues.

Historically, agricultural irrigation was the dominant use of water in these western rivers, and State agencies had responsibility for water administration to ensure that legal allocations of water were met. However, the ever-increasing population and accompanying need of water for commercial, industrial, and energy-related development is putting new stresses on the surface- and ground-water systems. As the demand for water increases for agricultural use and for other competing uses, planning and management are necessary for more resourceful use of water. While the need for planning and management has increased with time, the technology to improve planning and management also has increased. One of the most useful technological improvements has been the development of digital-computer models to simulate river-basin systems.

Several different models are available to simulate the hydrologic regime of such basins. The U.S. Army Corps of Engineers, Hydrologic Engineering Center, has developed a suite of models, including HEC III, HEC IV, and HEC V (U.S. Army Corps of Engineers, 1980) that are capable of simulating the quantity of flow in river basins. Another model developed by the U.S. Army Corps of Engineers, SSARR (U.S. Army Engineer Division, 1972), also has an interactive version, COSSAR. The U.S. Bureau of Reclamation also has developed river-basin simulation models such as HYDROSS (U.S. Bureau of Reclamation, 1978) and NW01 (Ribbens, 1975), which has the capability to simulate salinity (dissolved solids) in streams. Other available models include SIMYLD (Texas Water Development Board, 1972) and an interactive modification of that model, MODSIM (Shafer, 1979). All these models emphasize reservoir operations and many have varying degrees of optimization included, but none include the prior-appropriation allocation system explicitly, although some allow a priority for demands. A privately owned and operated model of the Colorado River basin in Colorado (Fleming, 1975) simulates the appropriation priorities to allocate water. None of these models attempt to simulate the ground-water/surface-water interactions explicitly. Taylor and Luckey (1972, 1974) developed a stream-aquifer model that used ground-water response functions to incorporate the time-delayed effects of ground-water pumpage and deep-percolation recharge of applied irrigation water on streamflow. Their model also used the prior-appropriation system established by Colorado water law to allocate the water of the Arkansas River basin in a 
reach from Pueblo to the Colorado-Kansas State line. Many other streamaquifer models are available that range from various types of response functions to standard ground-water-flow solutions. Most of these models are limited by the extent of the study areas, because of excessive computer resources needed for large basins.

A study was conducted by the U.S. Geological Survey, in cooperation with the Southeastern Colorado Water Conservancy District, to develop a model of the Arkansas River basin in Colorado. The model was to simulate the water quantity and quality of the Arkansas River, whose flow regime in the eastern one-half of the State is dominated by irrigation diversions and return flows. The irrigation diversions are determined by the prior-appropriation allocation system; and return flows, which greatly affect streamflow, result from the ground-water interactions of pumpage and excess irrigation applications. Thus, these functions had to be included in the model. A desired feature of the model was an interactive capability to facilitate use of the model by local water managers. The model described in this report was developed as part of this study.

The purpose of this report is to document the computer model. This documentation includes a description of the model capabilities and example inputs and outputs. Considerable information is included for potential model users, which are listed as Attachments at the back of the report. This information includes:

Attachment 1--Data instructions for streamflow and water-quality simulation.

Attachment 2--Example files of input data for streamflow and waterquality simulation.

Attachment 3--Data instructions for water-supply operations simulation.

Attachment 4--Example files of input data for water-supply operations simulation.

Attachment 5--Definition of commoned variables in subroutines for streamflow and water-quality simulation.

Attachment 6--Definition of commoned variables in subroutines for water-supply operations simulation.

Attachment 7--FORTRAN source code of subroutines for streamflow and water-quality simulation.

Attachment 8--FORTRAN source code of subroutines for water-supply operations simulation.

The computer code was designed for generic use--that is, for use in any river basin. Therefore the description of a river basin, its hydrology, and water use is entered completely through the input data. Because of the enormity and complexity of the Arkansas River basin, the basin described in this report is a hypothetical simplification for purposes of illustration. 


\section{DESCRIPTION OF THE MODEL FOR SIMULATING STREAMFLOW, WATER QUALITY, AND WATER-SUPPLY OPERATIONS}

Streamflow and its accompanying water-quality load (typically dissolved solids) are routed (accumulated) using a monthly time step. No hydraulic-flow routing is included in the model. The river basin is depicted in the model as a network of nodes. The network of nodes and sets of regression equations needed to compute streamflow and water-quality concentration are stored in a file known as the basin-description file. This file provides the spatial discretization and variability of the hydrology of the basin. The information in this file can be modified interactively during a simulation to produce results for different management alternatives. A second file, which is needed prior to simulation, is the time series of a set of independent variables used by the regression equations.

The simulation of water-supply operations and ground-water systems require additional description of the natural hydrologic system, such as evapotranspiration rates and initial ground-water storage and quality, which are stored in another data file. A file also is needed that lists the water users and their demand characteristics and supply sources. Although this file needs to be available prior to simulation, the information in the file also can be modified interactively during a modeling session to produce results for various management alternatives. If the supply sources include direct diversions, then a separate file, listing the ordered priorities of the allocation laws, needs to be available.

One of the major reasons a new model was developed for this project, rather than applying an existing model, was the desire to obtain results interactively on a computer-terminal screen. Graphical output was a feasible technique to summarize and collate the many values computed by such a simulation model. Two types of illustrations are available interactively during a simulation: (1) Schematic maps that depict areal information for fixed time periods; and (2) graphs that depict time information for fixed points. Also, time-series graphs and frequency curves can be drawn to compare results of the current simulation to results stored in other files from previous simulations. Such graphical output and other limited tables and summary statistics displayed interactively provide users with information that would be difficult to obtain by evaluation of large quantities of printed computer output. Many of the illustrations in this report are examples of these outputs; commonly, these illustrations would be displayed on the computer-terminal screen.

The model is written in FORTRAN, and every attempt is made to keep it generic for use on any computer or compiler. During different stages of development, the model was run on CYBER $^{1}$, AMDAHL, Honeywell Multics, and PR1ME computers. Minor modifications were necessary between computers to retrieve dates from the different computers and to handle a few character-string manipulations. The code presented in this report was used on a PRIME 9955 with the F77 compiler. All graphics use ISSCO DISSPLA. An initial version of

${ }^{1}$ Use of brand names in this report is for identification purposes only and does not constitute endorsement by the U.S. Gealogical Survey. 
the model was developed primarily to format data for initial development of the graphics options. That version had no capability to accumulate streamflow downstream and, thus, had little hydrologic-simulation capability. The part of the model used to simulate streamflow can be used alone without the wateruse and ground-water capabilities. Simple water-use systems can be included with the hydrologic-basin description by incorporating their effects in the regression equations. An evaluation of effects of coal mines on dissolved solids (Parker and Norris, 1983) was accomplished using this model without the water-use and ground-water systems.

\section{Algorithms for Streamflow and Water-Quality Simulation}

All of the hydrologic relations in the basin described by the model use regression equations. The incremental streamflow ( $\hat{Q}$ ) of each node in a basin (fig. 1) is determined by an algebraic equation with some independent variable. Three types of equations are available in the model to compute $\hat{Q}$ on the basis of a code for the independent variable:

$$
\begin{aligned}
& \hat{Q}_{i}=a+b X, \\
& \hat{Q}_{i}=a X, b, \text { and } \\
& \hat{Q}_{i}=a+b Q_{u},
\end{aligned}
$$

where $\hat{Q}_{i}$ is the incremental (or internode) streamflow at node $i$,

$a$ is the intercept-regression coefficient,

$b$ is the slope-regression coefficient,

$X$ is the independent variable, and

$Q_{u}$ is the streamflow at the upstream node.

For sites where measured data are to be used directly in the model, the $X$ values would be the measured streamflow, and the regression coefficients would be chosen as 1 and 0 . For sites where there is no measured data, or the length of record is insufficient, the $X$ values could be snowpack or precipitation or measured streamflow at some other site. The regression coefficients need to be determined by statistical methods separate from the model; however, they can be adjusted during the calibration process.

The resultant incremental streamflow could represent gains in flow due to tributary inflow, overland runoff, or irrigation return flow, or the resultant incremental streamflow could represent losses in flow due to diversions, bank storage, or evapotranspiration. For each month of the year, different types of equations, independent variables, and regression coefficients can be used.

The water-quality concentration ( $\hat{C})$ of each incremental streamflow also is determined by using regression equations, with the computed streamflow as the independent variable:

$$
\hat{\mathrm{C}}_{i}=\mathrm{cQ}_{\mathbf{i}}{ }^{\mathrm{d}} \text {, }
$$

where $\hat{C}_{i}$ is the concentration of the incremental streamflow $\left(\hat{Q}_{i}\right)$ at node $i$,

$d$ is the slope-regression coefficient.

As with the computation for streamflow, different regression coefficients for each month of the year can be used. 


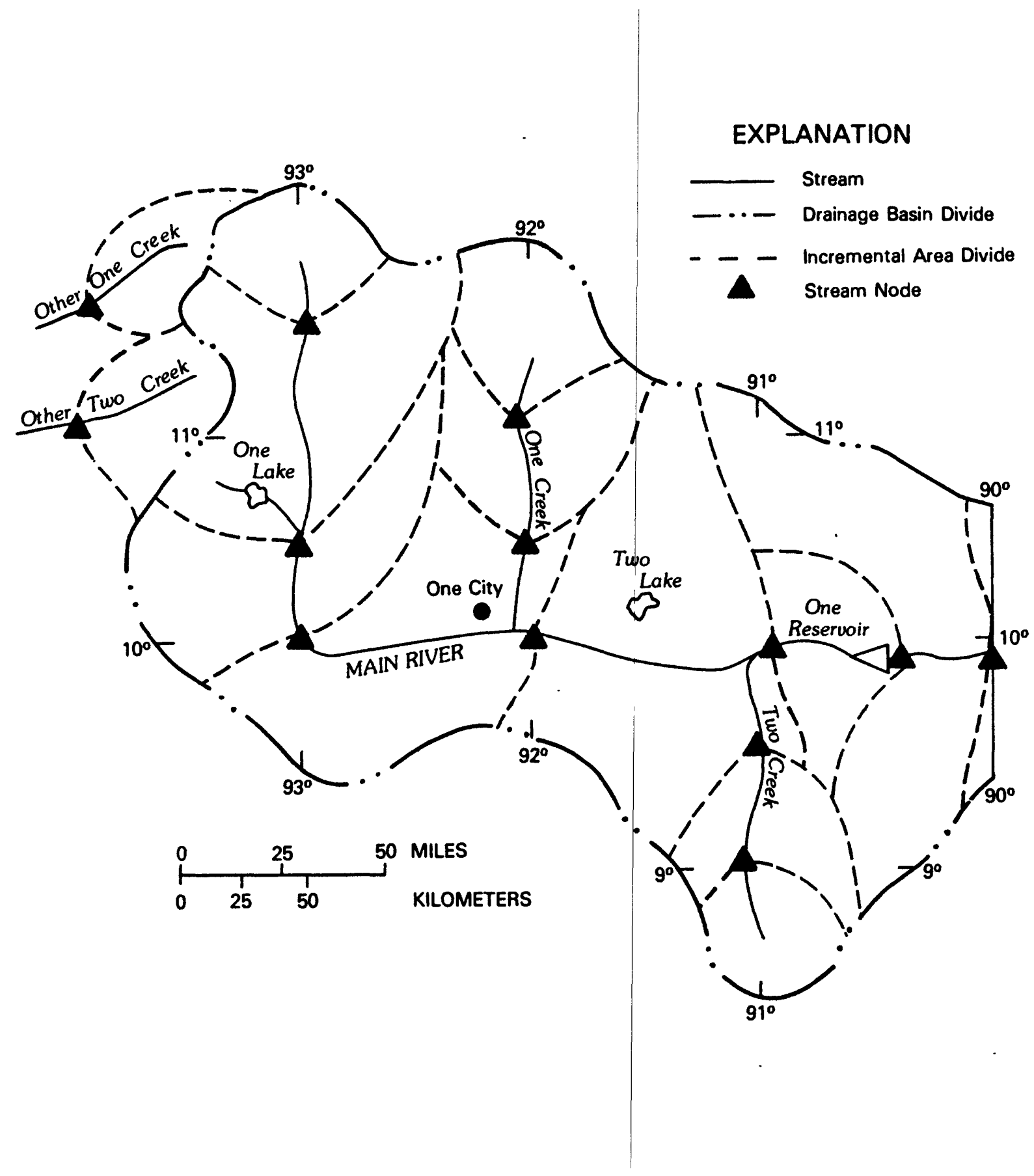

Figure 1.--Sample basin with nodes and incremental areas. 
The most commonly available water-quality data is specific conductance; thus, the $\hat{C}_{i}$ in equation 4 commonly would be specific conductance $\left(\mathrm{SC}_{\dot{f}}\right)$. To compute water-quality concentrations $\left(\hat{C}_{i}\right)$ from specific conductance $(\hat{S} C)$, another equation is used in the model:

$$
\hat{\mathrm{C}}_{i}=\mathrm{e}+\mathrm{f} \mathrm{SC}_{\mathrm{i}},
$$
where $\mathrm{SC}_{i}$ is the specific conductance of the incremental streamflow $\left(Q_{i}\right)$

$e$ is the intercept-regression coefficient, and

$f$ is the slope-regression coefficient.

This equation is used to convert specific-conductance values computed using equation 4 to dissolved-solids concentrations (or any other desired conservative constituent).

Total streamflow (Q) is computed as the sum of the streamflow of all immediately upstream nodes and the incremental streamflow:

$$
Q_{i}=\hat{Q}_{i}+\sum_{u=1}^{N} Q_{u} \text {, }
$$

where $Q_{i}$ is the streamflow at node $i$,

$Q_{u}$ is the streamflow at adjacent nodes immediately upstream from node $i$, and

$N$ is the number of adjacent nodes immediately upstream of node $i$.

The downstream water-quality concentration (C) is computed by dividing the sum of the loads $(Q C)$ reaching a node by the streamflow:

$$
c_{i}=\frac{\left[\hat{Q}_{i} \hat{c}_{i}+\sum_{u=1}^{N} Q_{u} c_{u}\right]}{Q_{i}},
$$

where $C_{i}$ is the concentration at node $i$, and

$\mathrm{C}_{\mathrm{u}} \mathrm{i}$ is the concentration at adjacent nodes immediately upstream

When the incremental streamflow is negative, a factor is specified to determine the concentration at the downstream node. This factor is determined during calibration; it adjusts the concentration to account for the quantity of the water-quality load that is lost when streamflow is lost. The two extreme possibilities are: (1) The loss of streamflow is the result of a diversion and, thus, downstream concentration remains equal to the upstream concentration; or (2) the loss of streamflow is the result of evapotranspiration with no loss of water-quality load and, thus, the entire upstream load becomes concentrated downstream. The factor allows a range between these extremes, equaling 0 for possibility 1 and equaling 1 for possibility 2 . 
An option in the model enables the computation of the total streamflow (Q) at any node directly rather than routing streamflow to a node. With the proper code for the independent variable, equations 1,2 , or 3 can be used to compute $Q_{i}$ rather than $\hat{Q}_{i}$. For this condition, equations 4 and 5 are used to compute $C_{i}^{i}$ rather than $\hat{C}_{i}^{1}$. This option is useful for entering measured data into the model format for plotting and for later comparisons during calibration.

\section{Model Components For Streamflow and Water-Quality Simulation}

A chart depicting the organization of the subroutines that constitute the FORTRAN program to simulate the streamflow of a basin without including the water-supply operations or ground-water systems is shown in figure 2. Each of the subroutines is described briefly in the following text. A complete listing of the FORTRAN source code is listed in Attachment 7.

Subroutine INSTRK merely displays, at the terminal, a complete set of instructions of the required input data, similar to those listed in Attachment 1 .

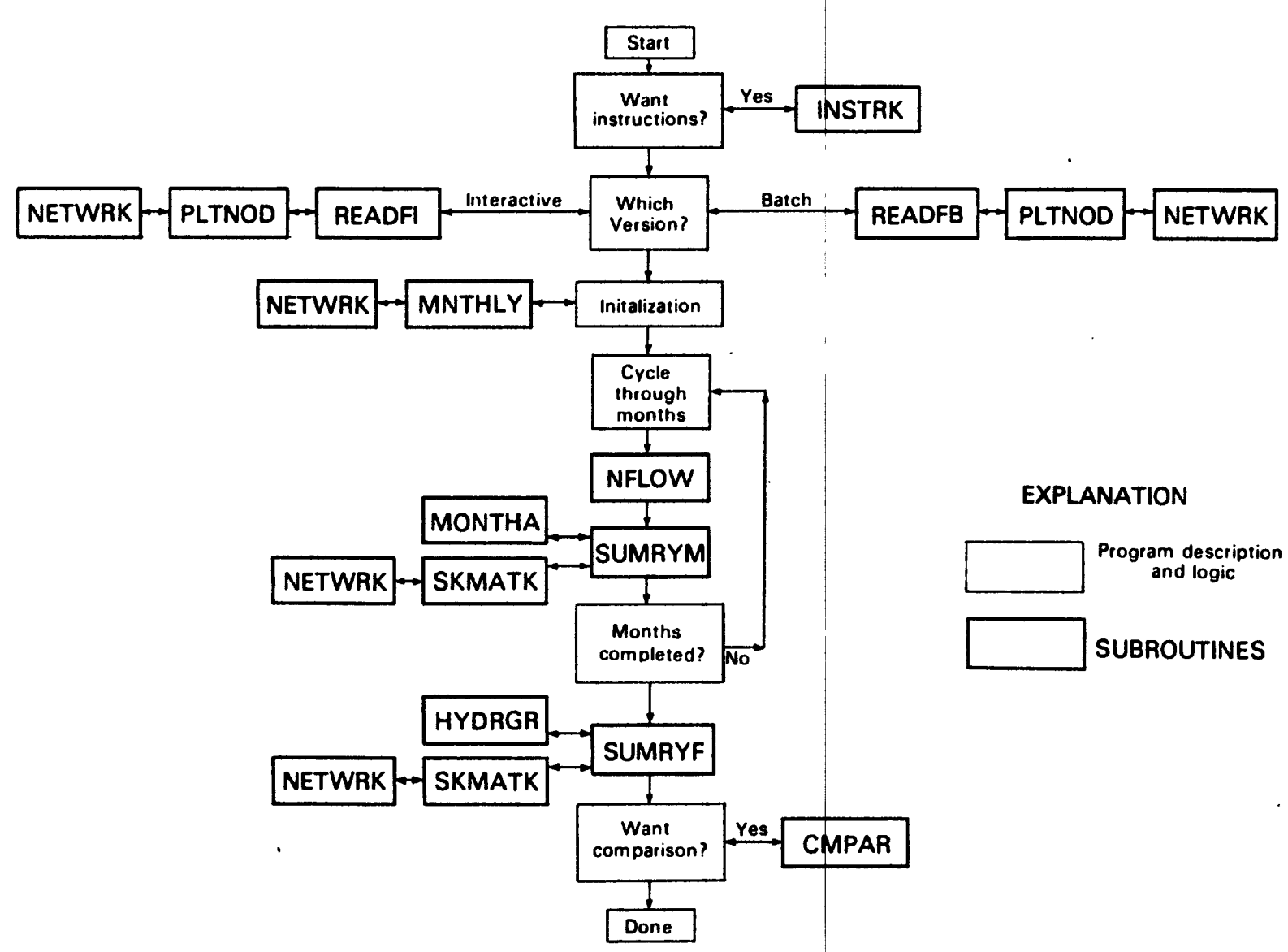

Figure 2.--Organizational chart of the subroutines used for streamflow and water-quality simulation. 
Either subroutine READFI or READFB is used to read the basin-description file into the computer from logical unit 60 . READFI is called when the model is operated interactively with certain information being supplied during the modeling session. READFB is called when the model is not used interactively and requires an additional data file (logical unit 64) that provides the needed information. The format of the basin-description file used by READFI or READFB is described in detail in Attachment 1 , and an example of such a file is shown in Attachment 2. For each node, the data include: (1) An identification number, (2) name, (3) location, (4) concentrating factor, (5) downstream-node identifier, and (6) slope- and intercept-regression coefficients for the relation of specific conductance to dissolved-solids concentration (eq. 5). For each node, there also is a set of monthly parameters including: (1) A code to determine which variable to use from the file of independent variables and what kind of relation (either eq. 1, 2, or 3) to use to compute the streamflow; (2) slope- and intercept-regression coefficients for streamflow (eq. 1, 2, or 3); and (3) slope- and intercept-regression coefficients for the water-quality concentration for any conservative constituent (eq. 4). READFI issues prompts for modifying data if any of the data read from the basin-description file are to be temporarily modified before the simulation is begun. Both READFI and READFB have the option of drawing a schematic node location (fig. 3), which is drawn by subroutine PLTNOD. From PLTNOD, subroutine NETWRK is entered to draw the drainage pattern of streams. The network of $x-y$ points that comprise the drainage pattern is read from a separate file using logical unit 62. The format for this digitized network of data is described in detail in Attachment 1, and an example of such a file is shown in Attachment 2.

Some accounting type variables are initialized in the mainline of the program. If a schematic of streamflow in the basin is to be updated monthly, then subroutine MNTHLY is called to draw the initial part of the schematic. From MNTHLY, subroutine NETWRK is called to draw the drainage pattern before hydrograph grids are drawn on the schematic within MNTHLY.

A loop in the mainline controls the monthly simulation. The major operative subroutine, NFLOW, is called in that loop. In NFLOW, the code for the independent variable for each node is deciphered to determine the type of relation and independent variable to be used to compute streamflow (eq. 1, 2, or 3). All the independent variables for that particular month are read from the independent-variable file (logical unit 61). The format of the independent-variable data is described in detail in Attachment 1 , and an example of such a file is shown in Attachment 2. Either the incremental streamflow or the total streamflow (depending on the type of relation chosen) is computed from the regression equation. Then the appropriate water-quality concentration is computed using the streamflow as the independent variable (eq. 4) and adjusting the concentration using a linear equation (eq. 5). The total streamflow at a node then is computed (eq. 6) as the sum of all streamflow from nodes immediately upstream added to the incremental streamflow. The waterquality load is routed similarly (eq. 7), by multiplying the streamflow of the upstream nodes by their respective water-quality concentrations and summing the loads to determine the load at the downstream node. For the special case when the incremental streamflow is negative (a losing reach), the routing of the water quality is computed by using a factor that would compute the waterquality concentration somewhere between the upstream concentration and the concentration that would occur if the total load were routed downstream. 
In the same monthly loop, subroutine SUMRYM is called. This routine updates all the summary statistics; stores the information for desired plots, tables, and statistics; and calls subroutine MONTHA, if the streamflow schematic is to be updated monthly. If a schematic of annual average streamflow is desired, subroutine SKMATK is called to generate that plot after simulating each December. The schematic of annual average streamflow (fig. 4) indicates the quantity of flow by the size of the symbol and prints the average flow for the particular year next to the symbol. The class interval of the waterquality concentration (normally dissolved solids) is indicated by the shape and color of the symbol. The monthly option for the streamflow schematic (fig. 5) is somewhat different from the schematic of annual average streamflow. Hydrographs are superimposed on the drainage pattern and are updated each month of the simulation, providing a movie-like effect of watching the symbols being plotted and erased and the hydrographs being drawn almost continuously while the model simulates each month of the given time period.

When the monthly loop for the period of record has been completed, subroutine SUMRYF is called. This subroutine generates summary schematics, hydrographs, and tables. If a schematic for average streamflow for the period of record (similar to fig. 4) is desired, then subroutine SKMATK is called from SUMRYF. If hydrographs are desired, then subroutine HYDRGR is called from SUMRYF to draw hydrographs of streamflow (fig. 6), water-quality concentration, and load. Generated within SUMRYF are tables of monthly streamflow (fig. 7), water-quality concentration, and load, and also a streamflow statistical summary (fig. 8).

At the completion of a simulation, a final subroutine is available that will plot and compare the streamflow, water-quality concentration, or load at any node, with the same parameter from some previous simulation. This comparison is done in subroutine CMPAR, which reads the output file created during this same simulation (logical unit 63) and some designated output from a previous simulation (logical unit 64). Three plots are generated for each comparison: (1) A histogram of both time series (fig. 9), (2) two frequency curves on the same plot (fig. 10), and (3) a histogram of the time series of the residual (or difference) between the two simulated results (fig. 11). The mean and standard deviations of the difference between the two simulated results are displayed. 


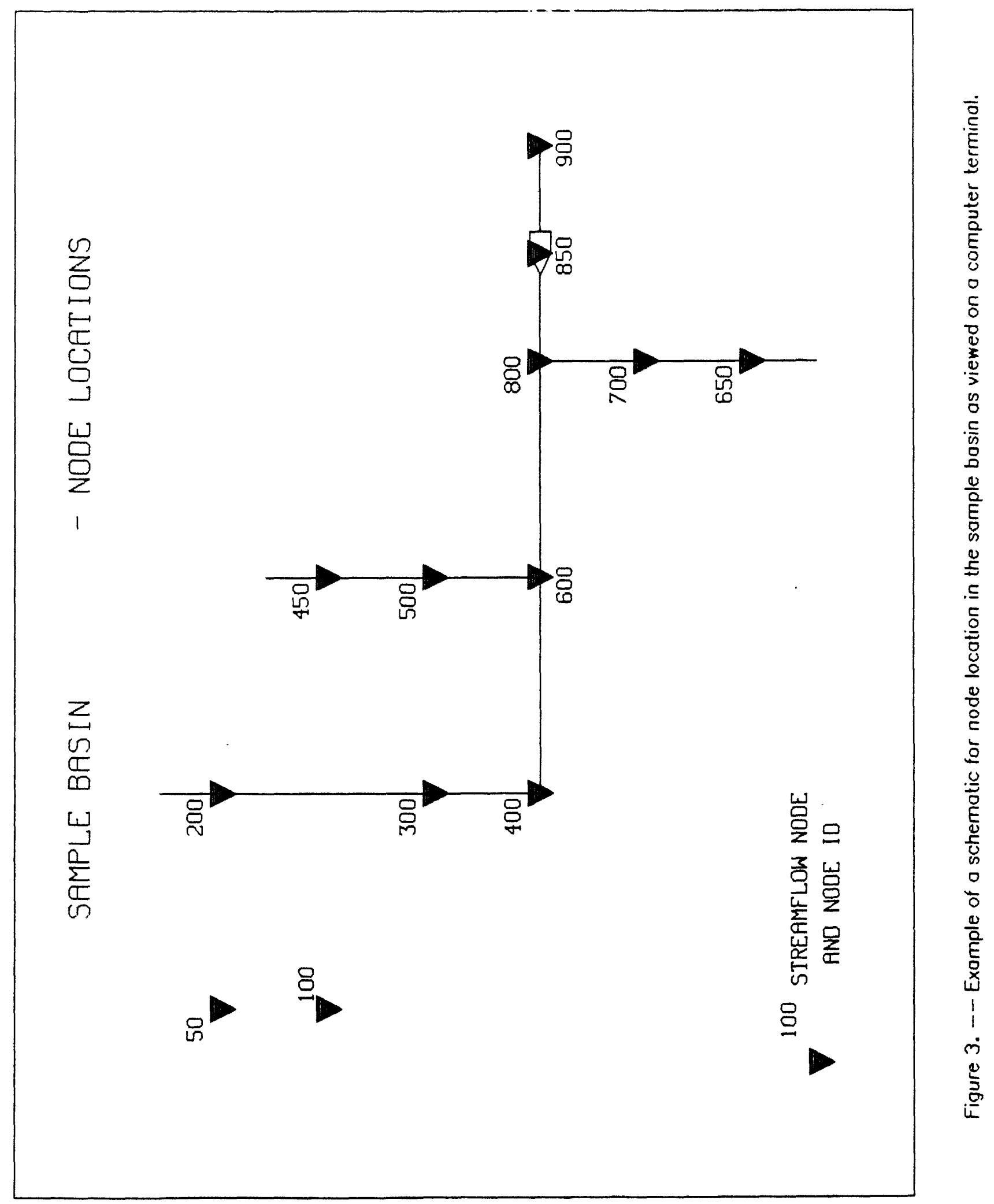




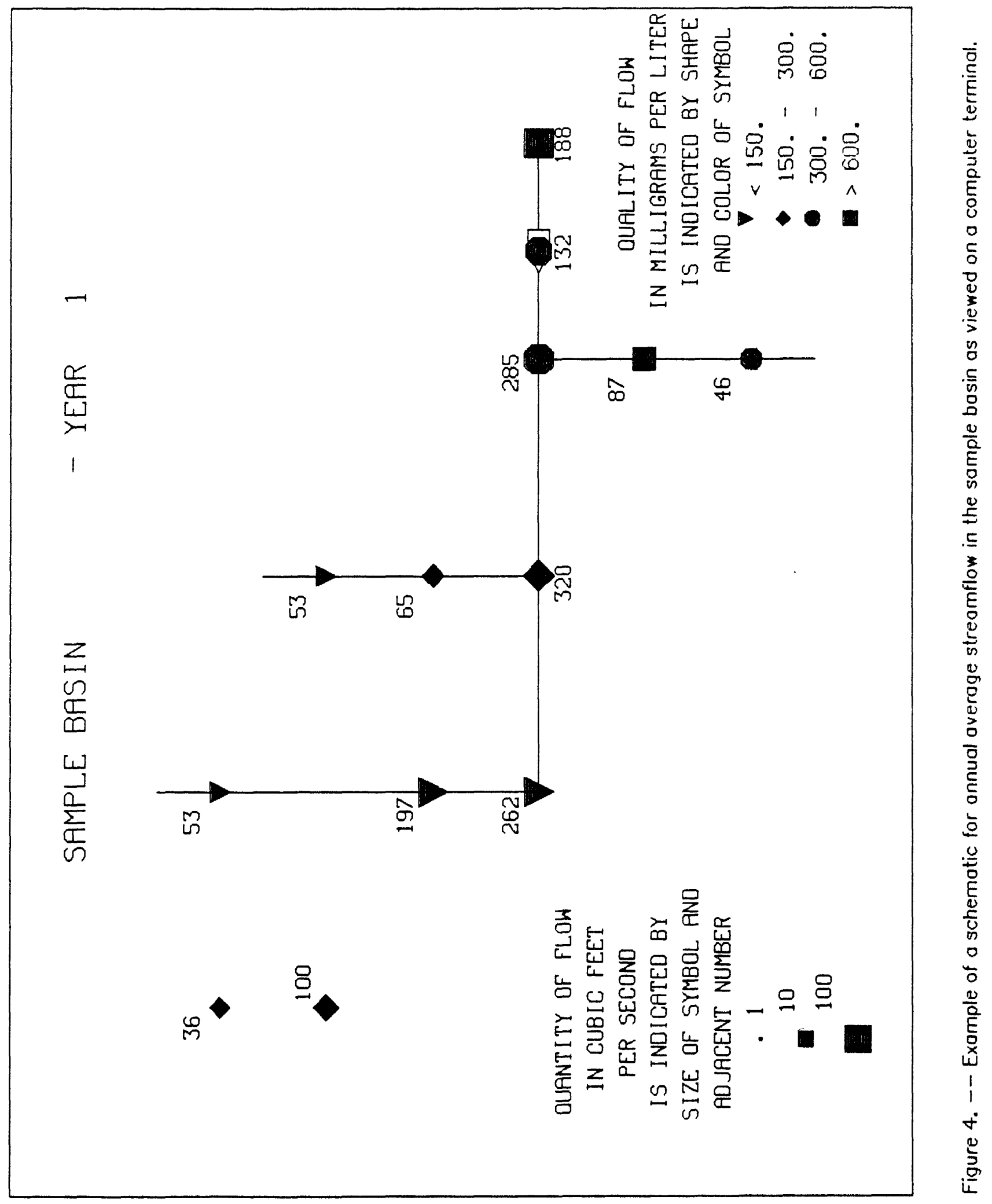




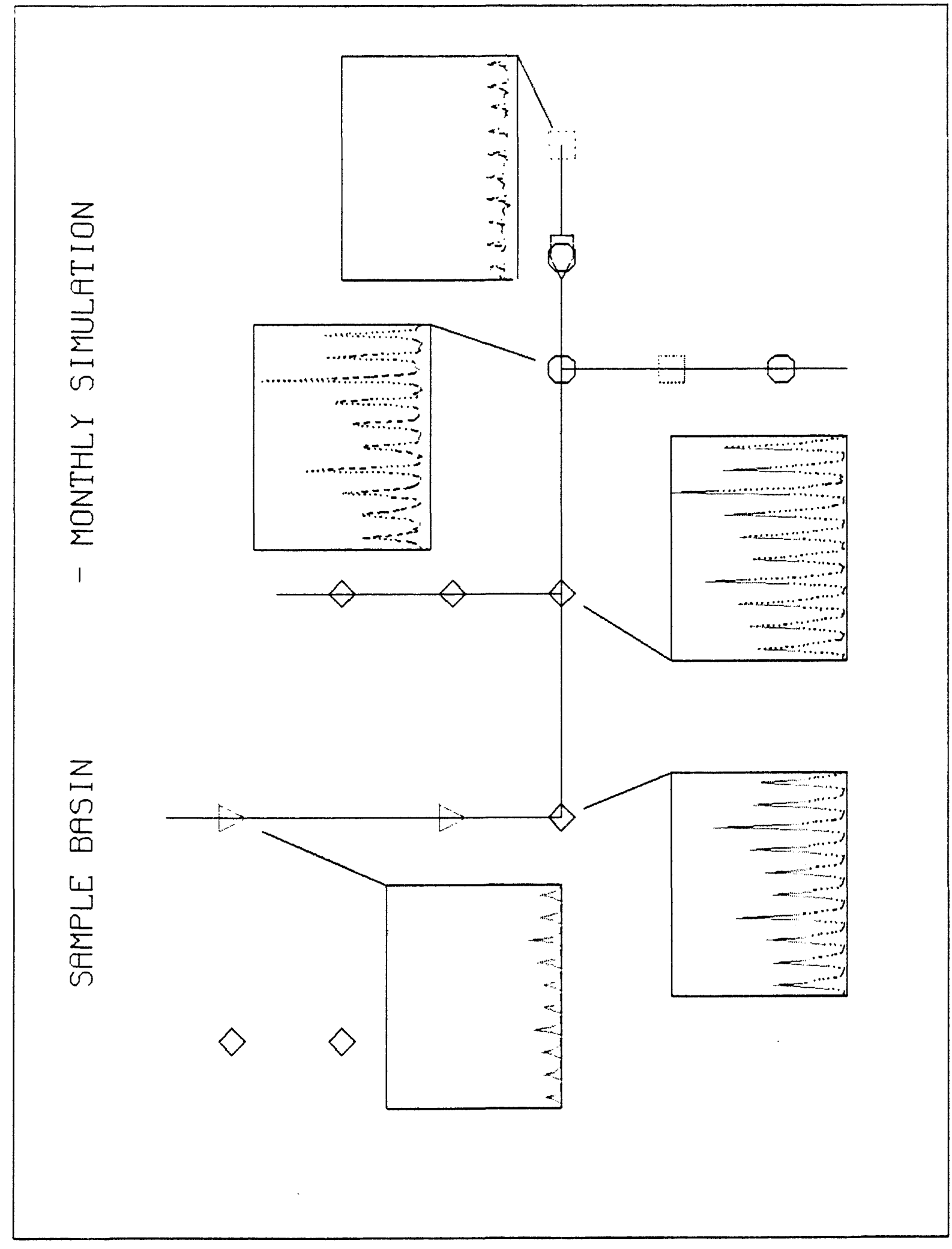

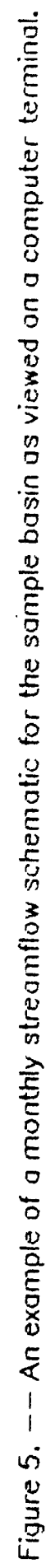




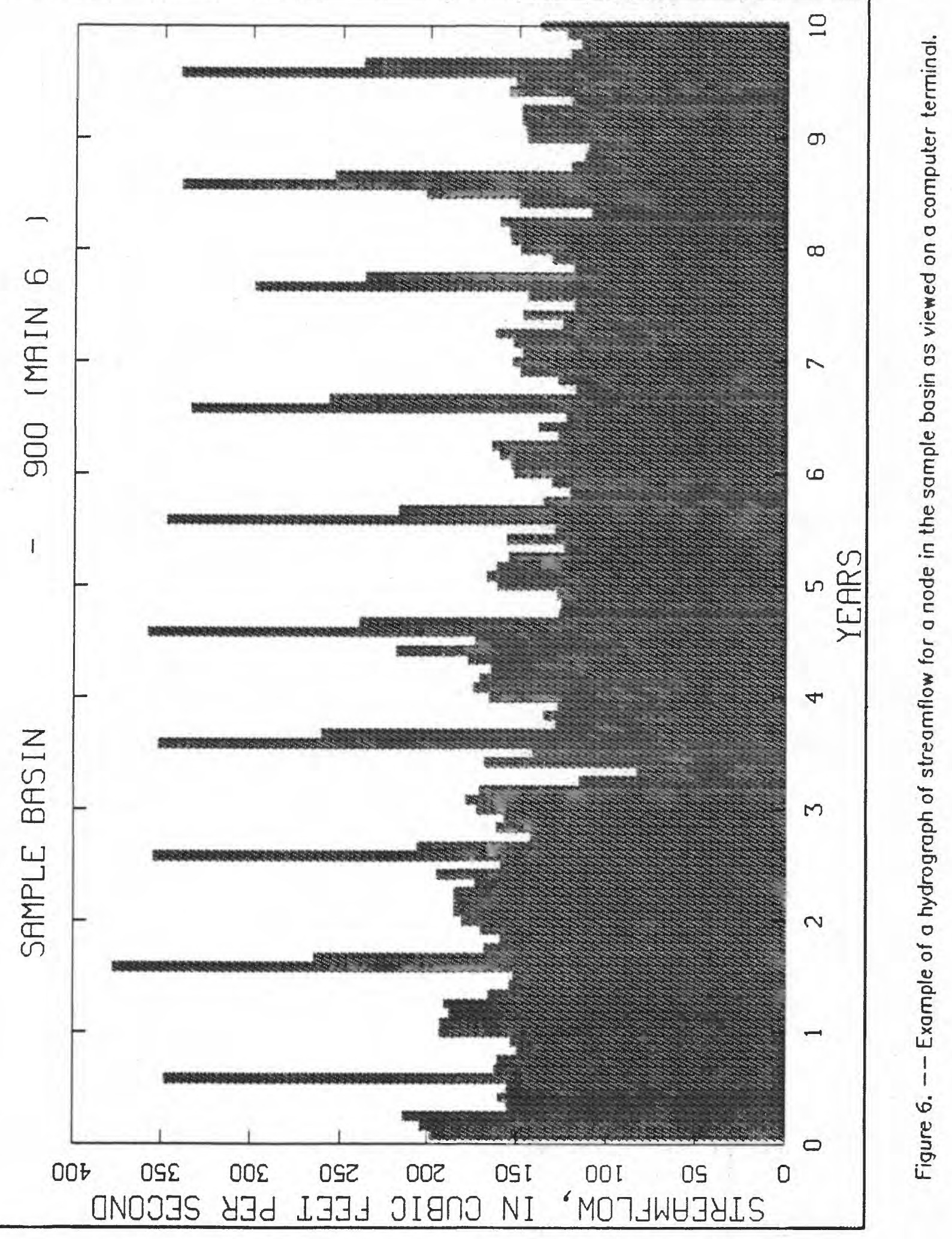


$0 \infty$ or $N \infty$ or $N$ b $N$

N

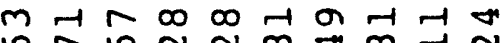

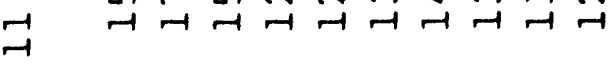

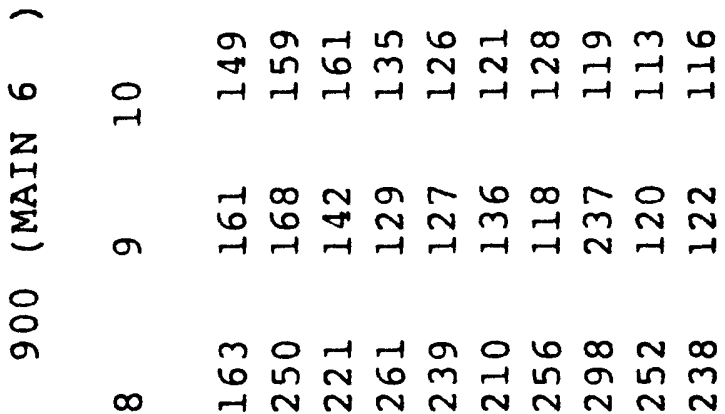

毼

$-1 \infty$ in $N$ a $\infty \pi+\pi$

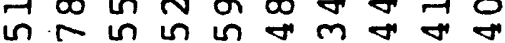

$n m m m m m m$

E

- $\quad n-1 \infty \pi n \infty N$

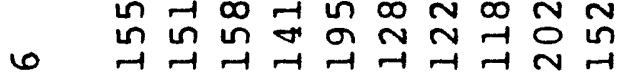

$\omega \pi \infty \infty$ or $m \infty \hat{n}$ or in

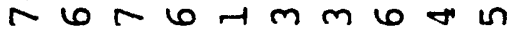

n $\rightarrow-H-N \rightarrow-1-1$

品

E-1

n 6 \% in

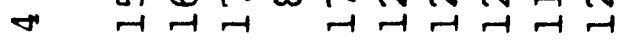

U $\quad \pi+\sigma N \infty \infty \infty \omega$

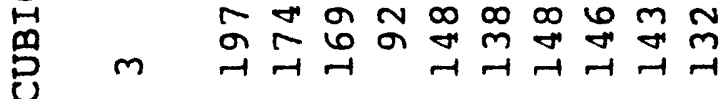

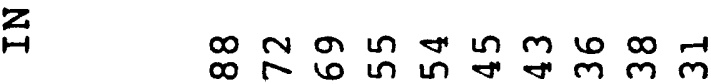

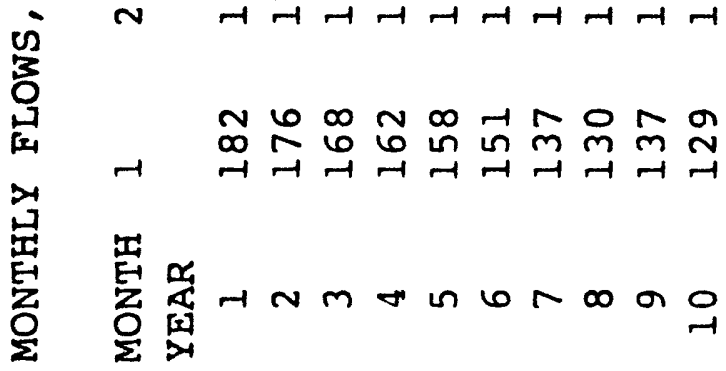




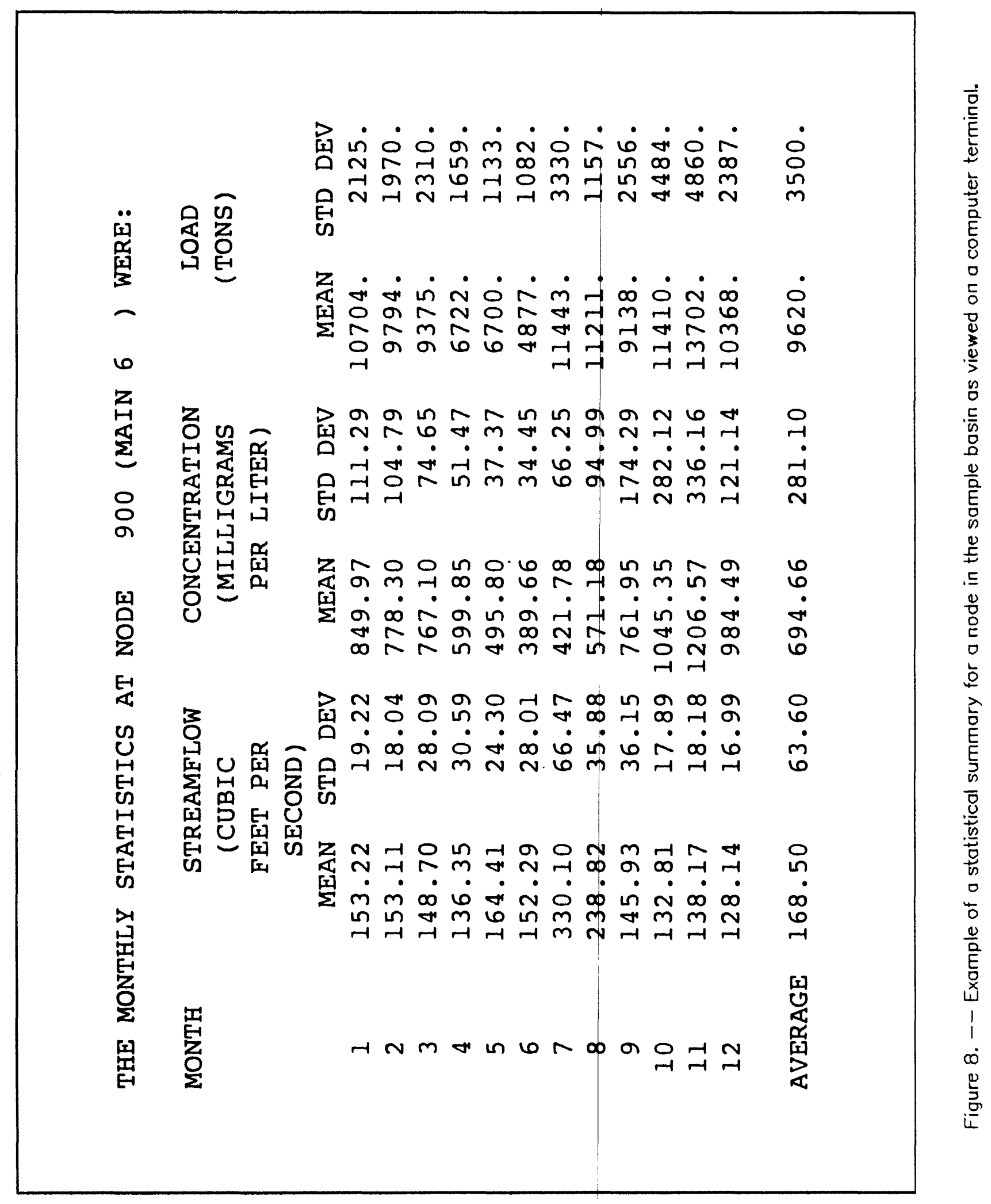




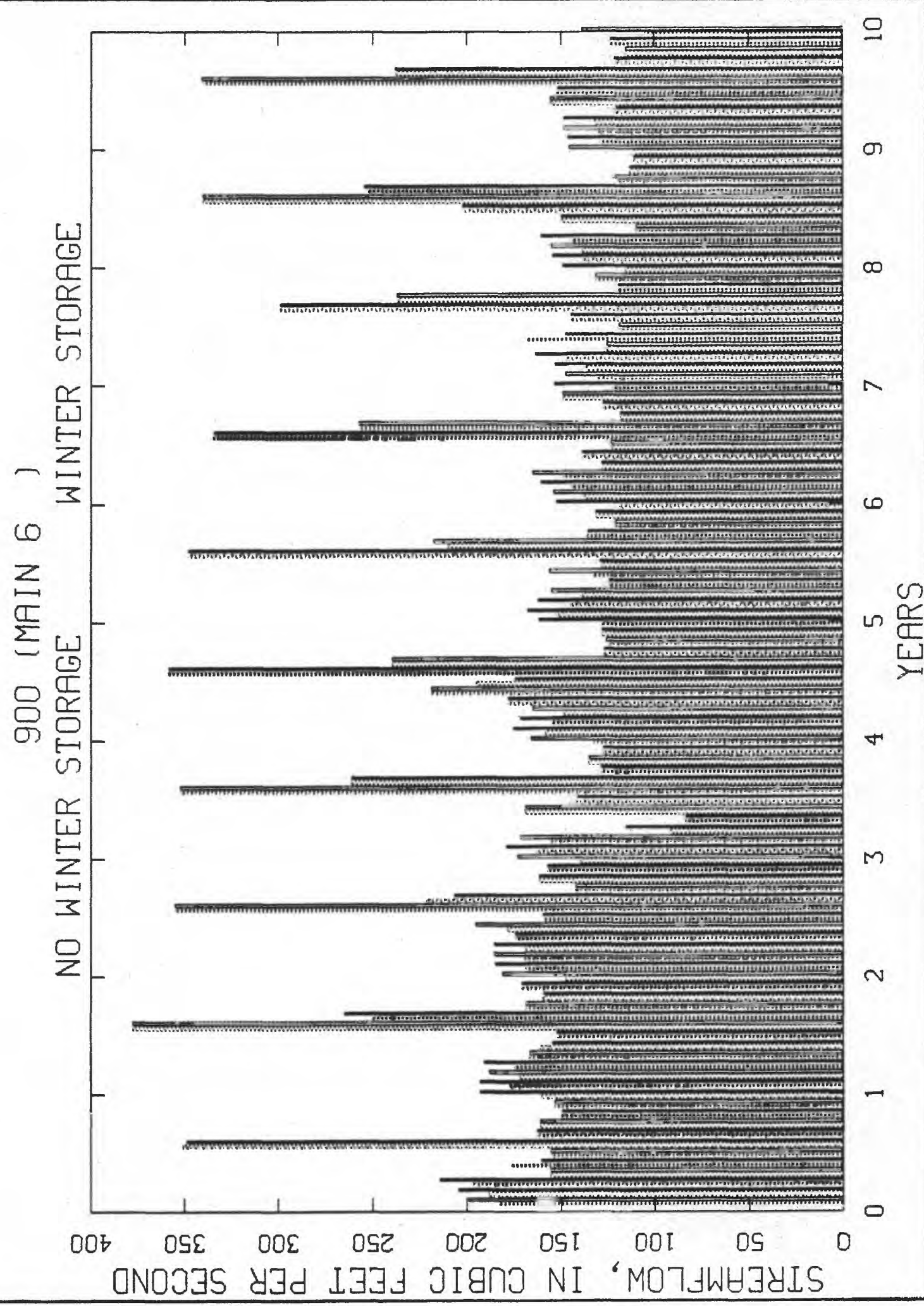

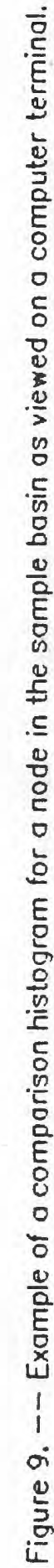




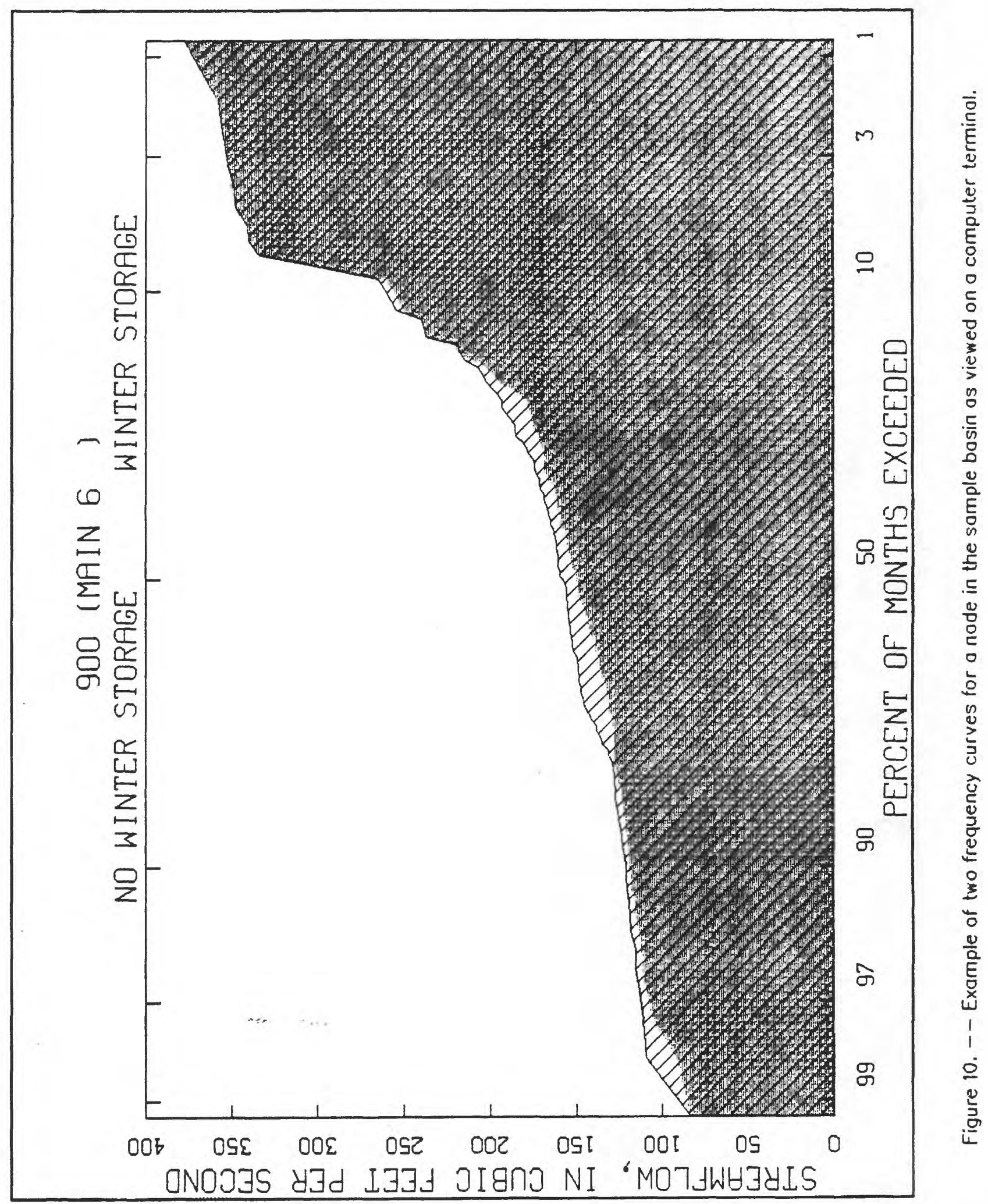




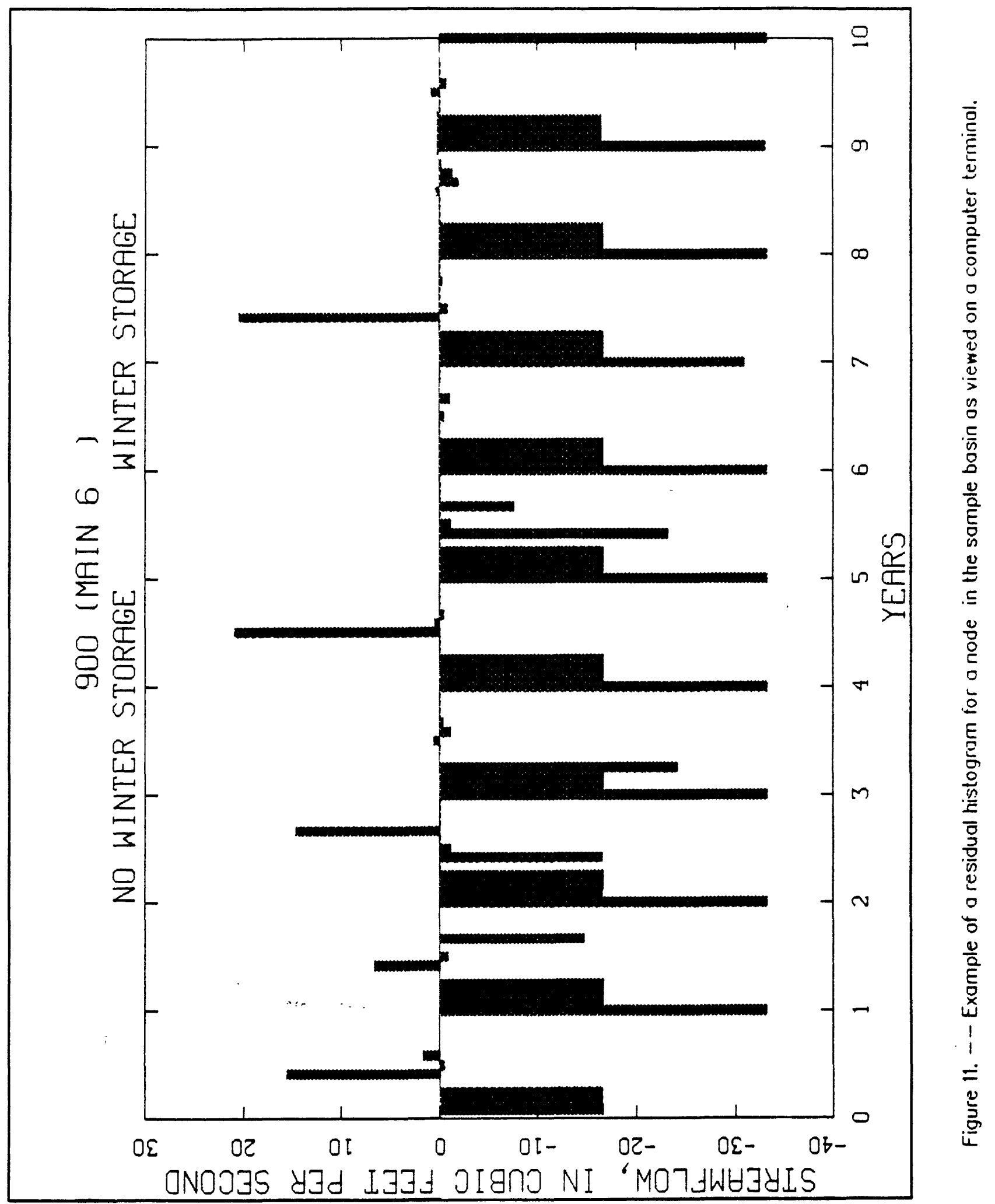




\section{Algorithms For Water-Supply Operations Simulation}

Inherent to including the water-supply operations to the description of a river basin is the inclusion of the ground-water system and reservoirs. Still needed are all the features to simulate the streamflow of a basin. The incremental areas of each node (fig. 1) still are used to simulate runoff from the basin; however, simulation of the water-supply operations (fig. 12) affects streamflow through reservoir operations and diversions, and simulation of the ground-water system allows the inclusion of pumpage and return flow from water use.

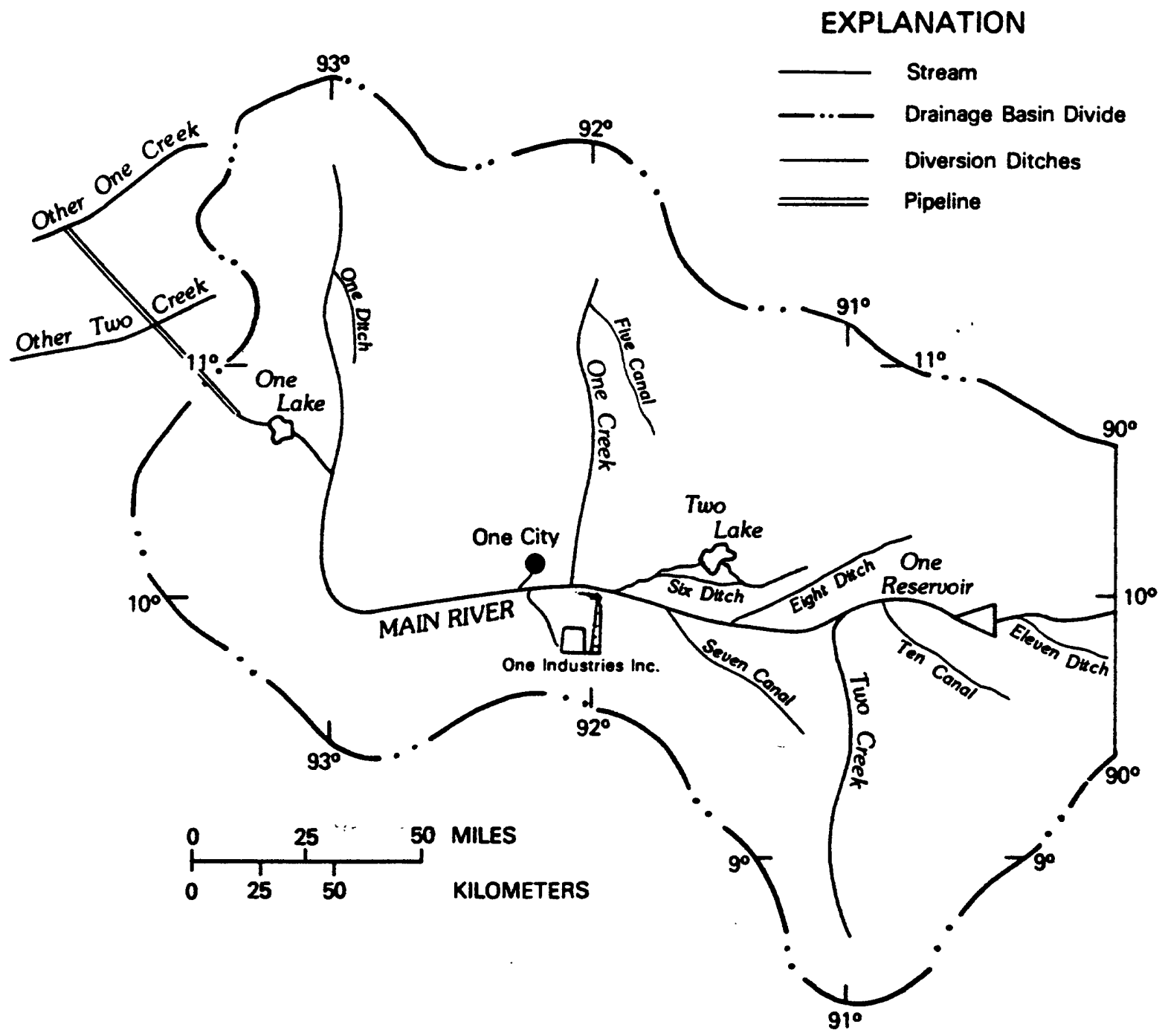

Figure 12.--Sample basin with diversion ditches and pipelines. 
Simulation of the water-supply operations is based on prioritized lists of sources of water. For every water user simulated, water need is computed based on the type of use and production level. The production-level categories for the different types of users are: (1) The number of irrigated acres for agricultural users, (2) the populace served for municipal users, (3) the number of units produced for industrial users, (4) actual water demand for instream users, and (5) the reservoir capacity for reservoir operators. A perceived water demand is computed for each user, using its water need and a demand factor. This demand factor is determined during calibration by comparing measured and simulated diversions. This factor has a decipherable code that causes the perceived demand to be based on the irrigation season only or on a separate monthly curve that computes demands throughout the year.

Each water user has a list of sources of water that can be used to satisfy the perceived demand. The list is in the order in which the user will use the sources. The types of sources of water available to a water user include: (1) Direct diversions, (2) ground-water pumpage, (3) reservoir releases, (4) imports (transbasin diversions), and (5) storage diversions. When direct and storage diversions are based on the prior-appropriation doctrine of western States water law, a separate list of water rights, in their basinwide prioritized order, is needed. The list of sources for each individual water user is compared against the basinwide list of water rights to ensure conformity to the law. The determination of how much water from a specific source will be used is based on the remaining unsatisfied demand, the physical availability of the water, the legal availability of the water, and the physical capacity for providing the water as each source is evaluated in its turn.

After the water deliveries to a user have been simulated, the model then simulates the fate of that water. Computations are based on the assumption that all delivered water first satisfies the water need. All delivered water that exceeds the water need becomes return flow. Return flow may be either tailwater, which as used in the model returns to the river in the month after being delivered, or deep percolation, which as used in the model returns to the river in a time-delayed function by using ground-water response curves. A factor, entered for each user, determines the percentage of the return flow that is tailwater and deep percolation to ground water. Canal leakage, which is subtracted from diversions before delivered water is computed, also is return flow and is distributed into tailwater and deep percolation to ground water by using the same percentage factor. A second type of return-flow computation that is designed for nonagricultural type users is available. For this type of computation, return flow is computed as a percentage of the delivered water. With this type of return flow, all of the computed return flow is assigned as either tailwater or deep percolation.

The ground-water system is simulated by use of ground-water response functions (Jenkins, 1968a) that compute the time-delayed effects of groundwater stresses on streamflow. Soil moisture is not simulated in the model; thus, all land-surface activities, such as ground-water pumpage, deep percolation of excess-water applications to ground water, and canal leakage, are assumed to affect ground-water storage immediately. The magnitude and timing of the effects of those stresses that are transmitted through the aquifer to the river are functions of the aquifer properties (transmissivity and storage coefficient) and the distance to the stream. Using the methods developed by 
Jenkins (1968b and 1968c) and more fully explained by Burns (1983, p. G2-G14), a unit-response function is computed by using volume-response functions:

$$
\frac{v}{Q t}=\left(\frac{x^{2} S}{2 T t}+1\right) \operatorname{erfc}\left(\sqrt{\frac{x^{2} S}{4 T t}}-\sqrt{\frac{x^{2} S}{4 T t}} \frac{2}{\sqrt{\pi}} e^{-\left(\frac{x^{2} S}{4 T t}\right)}\right),
$$

where $v$ is the volume of water removed from (or added to) streamflow,

$Q$ is the rate of stress on the aquifer,

$t$ is the time since the stress started,

$x$ is the distance from the stream to the stress point,

$S$ is the aquifer storage coefficient,

$T$ is the aquifer transmissivity, and

erfc is the complementary-error function.

Jenkins (1968a) developed the surrogate parameter, SDF (Stream-Depletion Factor), for use in realistic hydrologic settings to represent $x^{2} S / T$ in equation 8 , which is based on idealized aquifer conditions. Within the model, unit-response functions for five SDF values (fig. 13) are computed by subtracting a volume-response function (eq. 8) with the appropriate parameters from the same volume-response function lagged in time by 1 month. Thus, whenever a stress is simulated within the model, that stress is multiplied by the appropriate unit-response function and all future effects of that stress on streamflow are stored for eventual use. The assumption of one uniform value for storage coefficient and transmissivity for the entire basin is incorporated in the model. Distances for the point of deep percolation and ground-water pumpage for each user are computed, and used with the storage coefficient and transmissivity to determine which of the SDF groups are to be used. Rather than using the computed distances, distances can be entered and used to compute other SDF values to compensate for irregular values of storage coefficient or transmissivity.

\section{Model Components For Water-Supply Operations Simulation}

A chart depicting the organization of the subroutines constituting the FORTRAN program to simulate the water-supply operations in a basin is shown in figure 14. Each of the new subroutines (some shown on fig. 14 are used for the streamflow and water-quality simulation) is described in the following text. A complete listing of the FORTRAN source code is in Attachment 8 .

Subroutine NSTRK2 merely displays, at the terminal, a complete set of instructions of the required input data, similar to those listed in Attachment 3.

Either subroutine READMI or READMB is used to read the additional basindescription file into the computer from logical unit 53. READMI is called when the model is operated interactively with certain information being supplied during the modeling session. READMB is called when the model is not used interactively and requires an additional data file (logical unit 64) that provides the needed information. The format of the additional basindescription file used by READMI or READMB is described in detail in Attachment 3, and an example of such a file is listed in Attachment 4. Those data include monthly rates of crop potential evapotranspiration, lake evaporation, agricultural-irrigation demand, and instream-water demand. Other needed data 


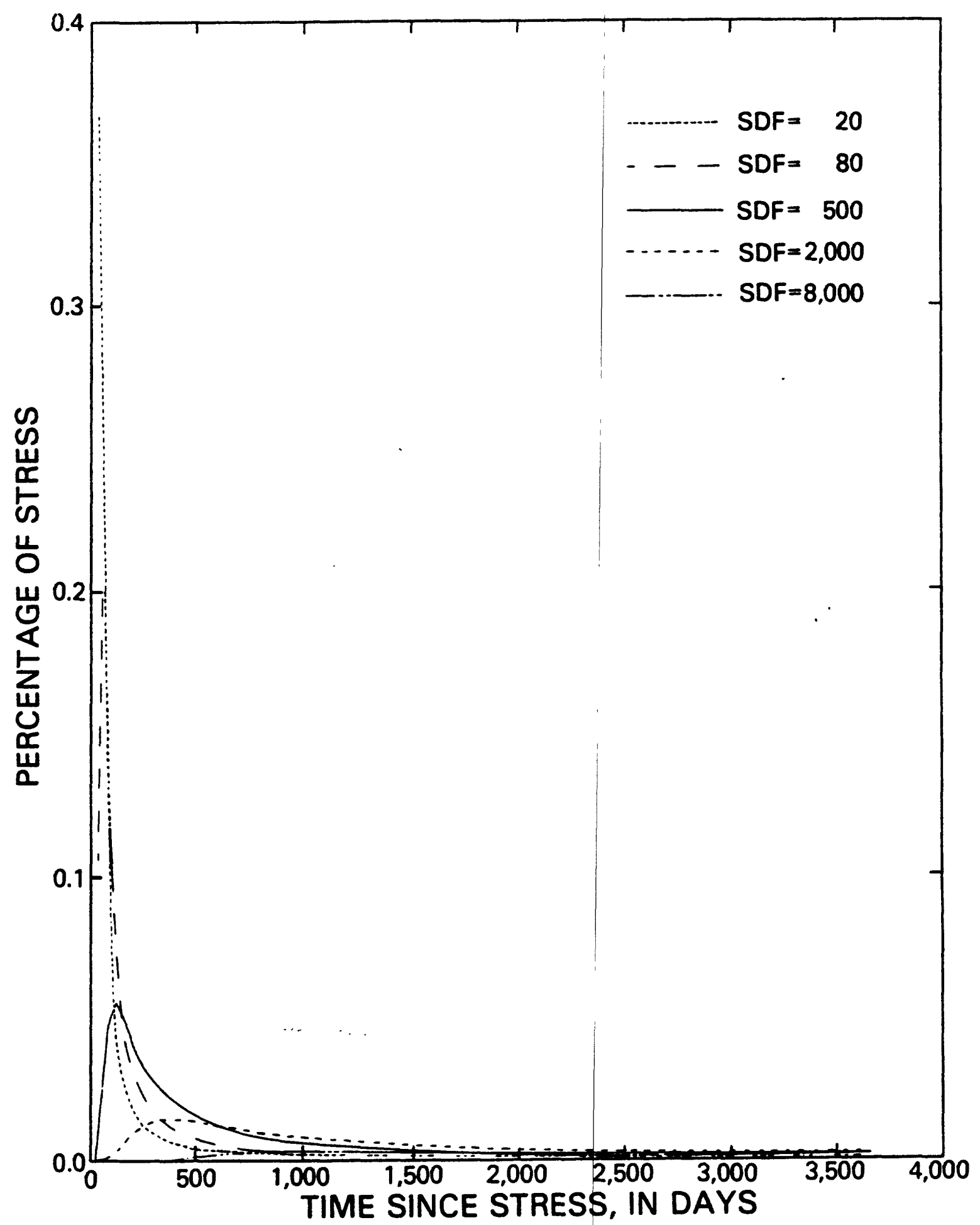

Figure 13.--Unit-response functions for five selected stream-depletion factors (SDF). 


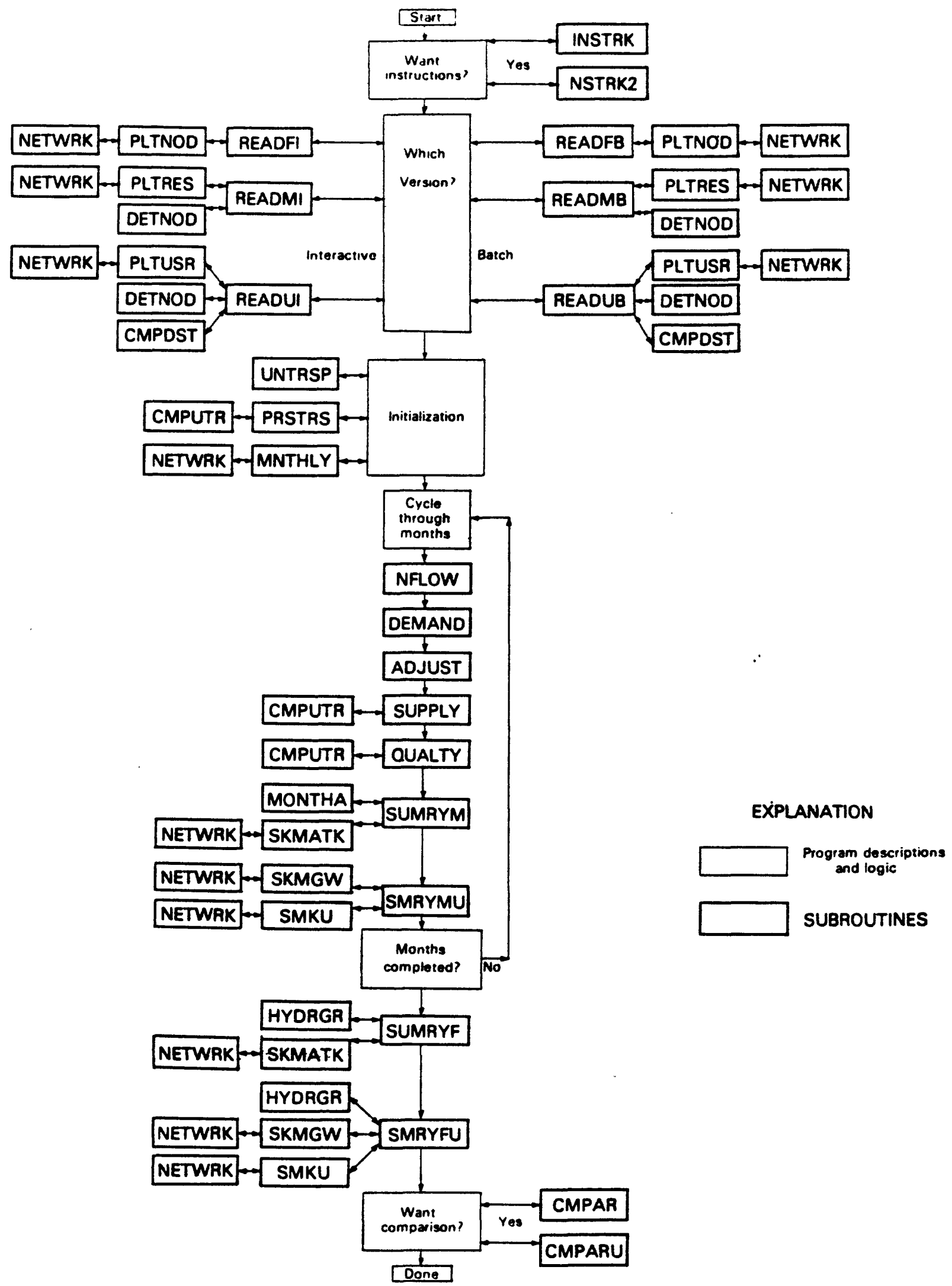

Figure 14.--Organizational chart of all model subroutines. 
include the average water demand per person and per unit produced, the basinwide average transmissivity and storage coefficient, and canal-leakage rate. Several factors and parameters needed by the model are entered through one of these subroutines. The length of each river reach is computed from the latitude-longitude location of each node, converting degrees to miles by using a conversion parameter, and sinuosity parameters read in as data. An effective precipitation factor identifies how much of the monthly precipitation falling on an irrigated area effectively contributes to plant consumptive use. The rest of the precipitation is not included in the model, except as the incremental streamflow may be based on precipitation. Finally, the model is prestressed to generate initial return flows. A prestress factor, determined during calibration, needs to be entered through one of these subroutines. All of the physical data for the reservoirs are entered into the model through either READMB or READMI. The needed data for each reservoir includes: (1) An identification number, (2) name, (3) location, (4) maximum and inactive capacities and surface area, and (5) initial contents and water-quality concentration. Also entered through one of these subroutines is the initial quantity and quality of ground water in storage for each side of the river upstream from each node. READMI issues prompts for modifying data if any of the data read from the additional basin-description file are to be temporarily modified before the simulation is run. READMI and READMB have the option of drawing a schematic for reservoir location (fig. 15), which is drawn by subroutine PLTRES. The size of the symbols correspond to the size of the reservoirs. From PLTRES, subroutine NETWRK is entered to draw the drainage pattern of streams.

The water-supply operations file is entered into the model through either subroutine READUI or READUB from logical unit 50. READUI is called when the model is operated interactively with certain information being supplied during the modeling session. READUB is called when the model is not used interactively and requires an additional data file (logical unit 64) that provides the needed information. The format of the water-supply operations file used by READUI or READUB is described in detail in Attachment 3, and an example of such a file is listed in Attachment 4. For each water user, those data include: (1) An identification number, (2) name, (3) location, (4) type of user, (5) quantity of use, (6) demand factor (relating to the perceived demand), (7) number of sources, and (8) type, percent, location, and distance of return flow. Also needed for every source of each water user is: (1) Type, (2) quantity available, and (3) location. The sources of water need to be entered in the order in which the water user is to use the sources. READUI issues prompts for modifying data if any of the data read from the watersupply operations file are to be temporarily modified before the simulation is begun. The prioritized basinwide water-rights list also is entered into the computer by one of these two subroutines from logical unit 51. The needed data include: (1) Water-user identification number, (2) name, (3) quantity, and (4) priority date of each water right. Both READUI and READUB have the option of drawing a schematic of water-user location (fig. 16), which is drawn by subroutine PLTUSR. The size of the symbols corresponds to the quantity of use for each user. Also, the lines indicate where a diversion is made, where ground water is pumped, or where excess applications recharge the ground-water systems. From PLTUSR, subroutine NETWRK is entered to draw the drainage pattern of streams. 


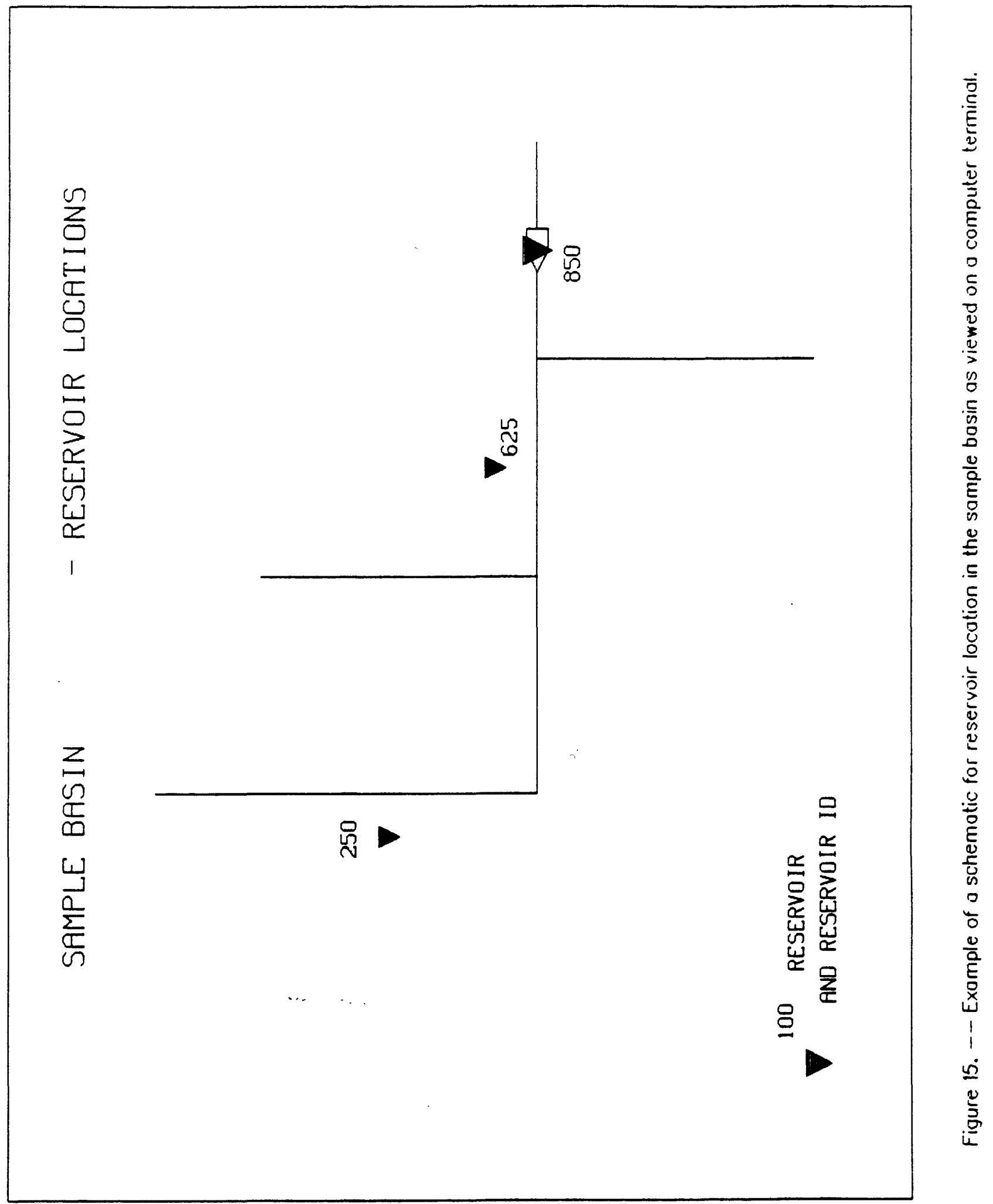




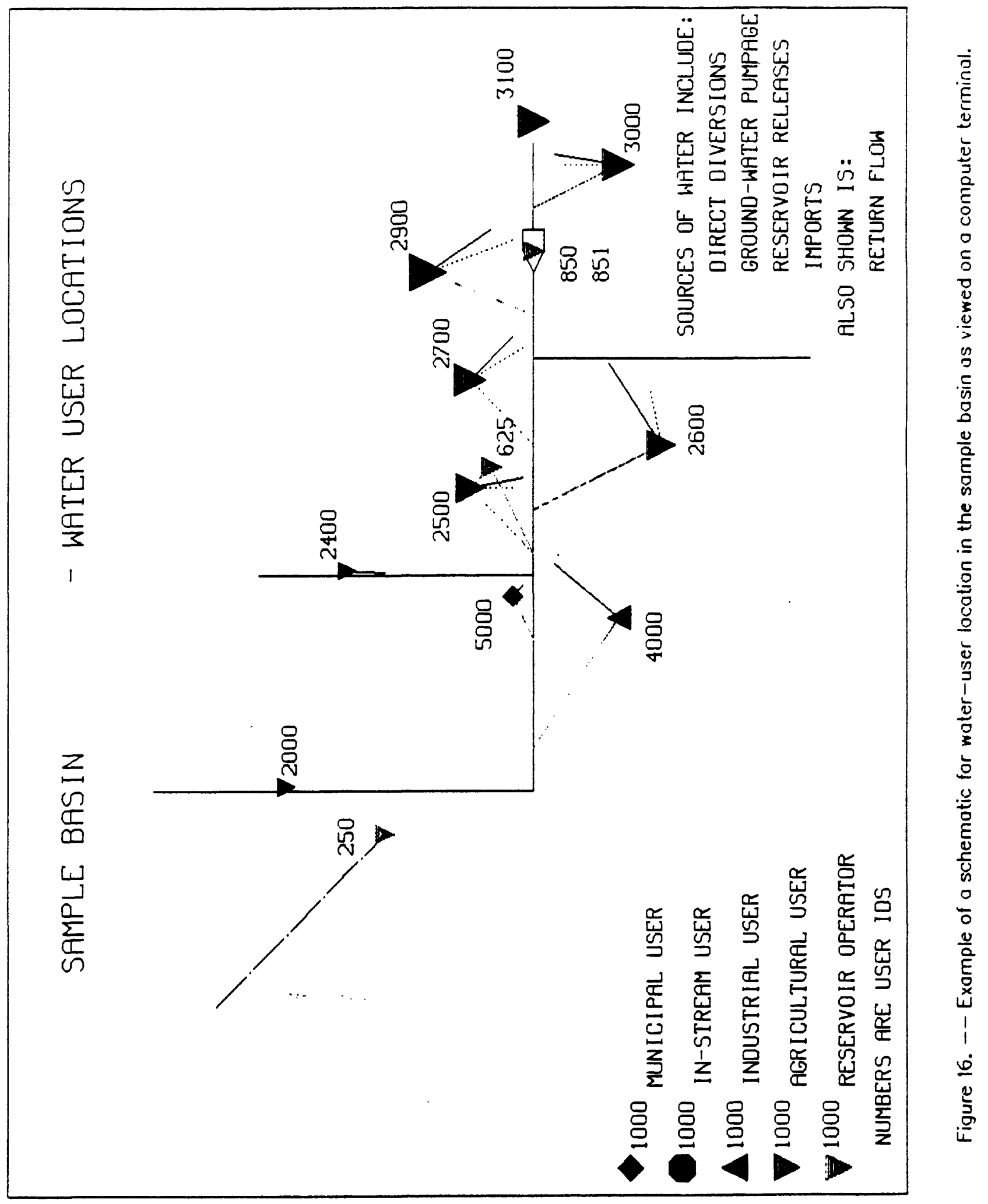


Some accounting-type variables are initialized in the mainline of the program. If a streamflow schematic is to be updated monthly, then subroutine MNTHLY is called to draw the initial part of the schematic. From MNTHLY, subroutine NETWRK is called to draw the drainage pattern before hydrograph grids are drawn on the schematic within MNTHLY. The unit-response functions necessary for the simulation of the effects transmitted through the groundwater system need to be initialized. The unit-response functions for five SDF values are computed in subroutine UNTRSP. Each unit-response function is computed as the difference of two volume-response functions (computed using eq. 8 ), one of which is lagged 1 month behind the other. A final initialization step required is to prestress the model. This process, done in subroutine PRSTRS, generates reasonable ground-water return flows representative of historic usage; thus, when the simulation begins, the initial return flows are not all zero. Within this subroutine, 10 years of return flows, as computed by using the water-user needs multiplied by the prestress factor, are simulated to stress the aquifer. For each user and for each month of the 10-year prestress period, subroutine CMPUTR is called to multiply the assumed stress by the appropriate unit-response function.

A loop in the mainline then controls the monthly simulation. NFLOW, as described in the previous section for simulation of streamflow, is entered to generate the natural streamflow available for water use. Next, the perceived water demand for each water user is computed in subroutine DEMAND. This demand is computed based on the type of water use and the specified demand factor. This perceived demand is the driving force within the simulation model because the model attempts to obtain all the water it can from each available source until the perceived demand is satisfied.

Within subroutine ADJUST, the flows computed in NFLOW are adjusted to account for return flows generated from previous water-use activities. Similarly, ground-water storage is adjusted for those same return flows. Reservoir evaporation also is computed in this subroutine.

The next subroutine within the monthly simulation loop is SUPPLY, which simulates the water-use system. The program loops through the basinwide water-rights list to: (1) Determine which user owns the next right, (2) determine whether that user has a perceived demand for water, (3) determine if that user has other water sources that are higher in the user's priority system, and (4) determine whether sufficient water is in the river to make the diversion. If the user has other, higher priority sources of water, those sources are simulated before using the direct diversion. For direct diversions, the quantity of the diversion is defined as the minimum of: (1) The perceived demand, (2) the quantity of the water right, or (3) the legal quantity of water in the river. After a nonzero diversion has been computed, all streamflow (both upstream and downstream) is adjusted, so that no subsequent diversion can affect this more senior diversion. The adjustment is transferred upstream until sufficient inflow equal to the diversion is determined and is transferred downstream to the end node or until the river is dried up at some intervening node. After going through the entire basinwide water-rights list, the program then goes through the 1 ist of all water users to determine if any other sources of water exist in each individual priority list that have not been used in an attempt to satisfy the perceived demand. 
The last operative subroutine in the monthly simulation loop is QUALTY. This subroutine simulates the streamflow similar to NFLOW, but also simulates the water-quality mixing at the same time. However unlike NFLOW, the return flows computed in ADJUST and the reservoir releases and stream diversions computed in SUPPLY are included in the simulation. The reservoirs also are simulated in this subroutine using inflows and releases computed in SUPPLY and properly accounting for water-quality mixing. Finally, each water user is simulated by computing the water quality of the applied water using the quantities from various sources computed in SUPPLY and the respective waterquality concentrations computed earlier in this subroutine. After computing the quality of the applied water, the quality of all return flows and ground water is computed. The concentration of return flow is computed by dividing the total load applied to the land surface by the quantity of the return flow; this accounts for the concentrating effects of the crop consumptive use of water. Ground-water concentrations are then computed by mixing the return flow with the ground water previously in storage.

The monthly-simulation loop is completed by calling the statisticalsummary subroutines SUMRYM and SMRYMU. SUMRYM is the same as described for the streamflow simulation. Within SMRYMU, statistics for ground-water storage, water use, and reservoirs are summarized and stored. If a schematic for annual ground-water storage is desired, subroutine SKMGW is called from SMRYMU to generate that schematic after each December has been simulated. Similarly, if a schematic for annual, average water-use is desired, subroutine SKMU is called from SMRYMU to generate that schematic after each December has been simulated.

When the monthly loop has been completed for the period of record, subroutines SUMRYF and SMRYFU are called. SUMRYF is the same as described for the simulation of streamflow. SMRYFU summarizes all of the ground water, water use, and reservoirs. If a schematic for final ground-water storage (fig. 17) is desired, subroutine SKMGW is called from SMRYMU. If a schematic for period-of-record average water use (fig. 18) is desired, subroutine SKMU is called from SMRYMU. Within SMRYFU, the ground-water summaries are generated first. Ground-water storage, ground-water concentration, and return flows for any river reach and side can be displayed as hydrographs (similar to fig. 6) or as tables (similar to fig. 7). The same parameters, summed for the entire basin, also are available as hydrographs or tables. Summaries available for any water user include direct diversions, ground-water pumpage, and total applications, which are displayed as either hydrographs (similar to fig. 6) or tables (similar to fig. 7). A statistical summary (fig. 19) also is available for any water user. Any of these summaries also can be displayed for the basinwide total water use. Finally, reservoir contents or waterquality concentration can be displayed as hydrographs (similar to fig. 6) or tables (similar to fig. 7). A statistical summary for a reservoir (fig. 20) also can be displayed. 


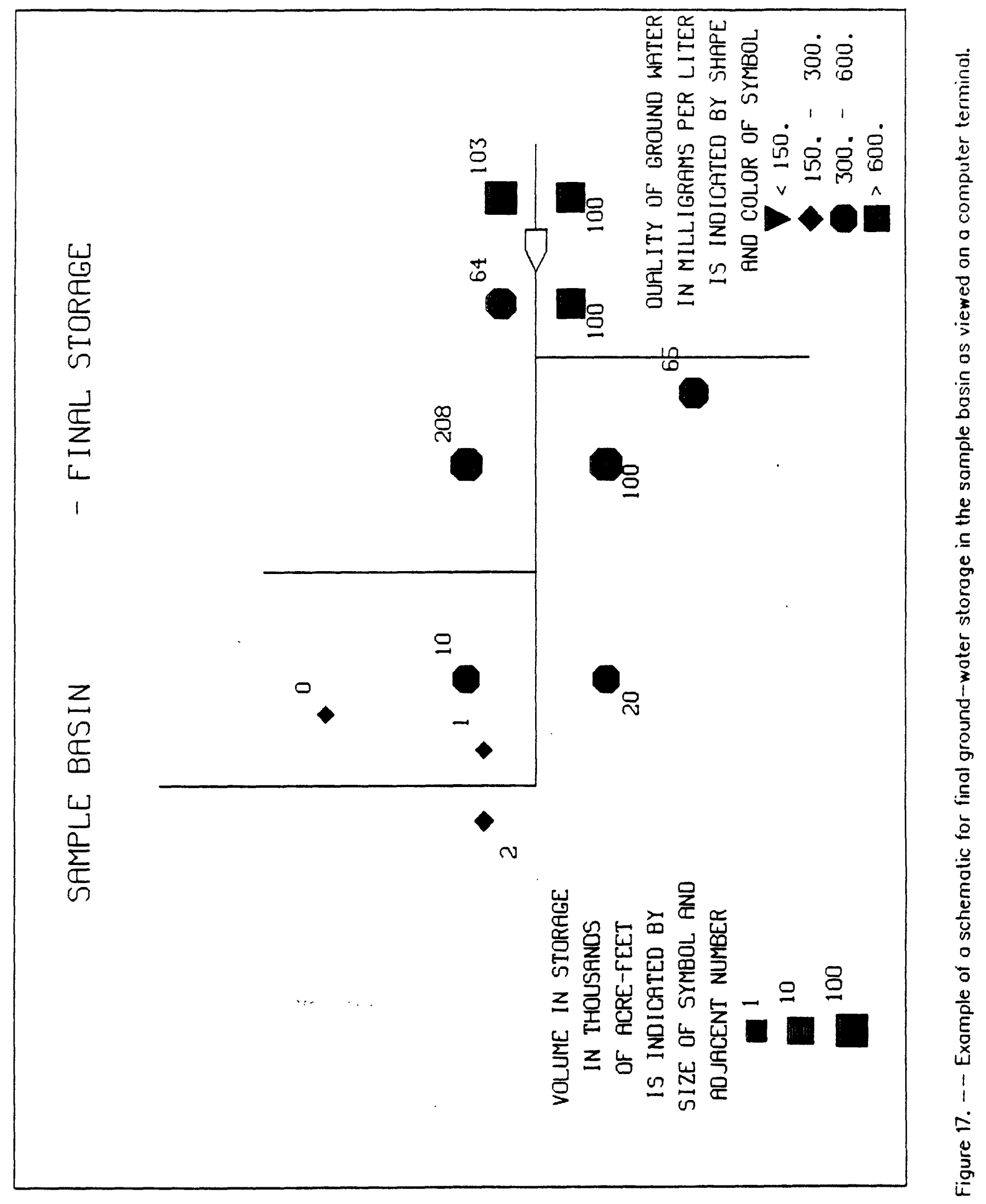




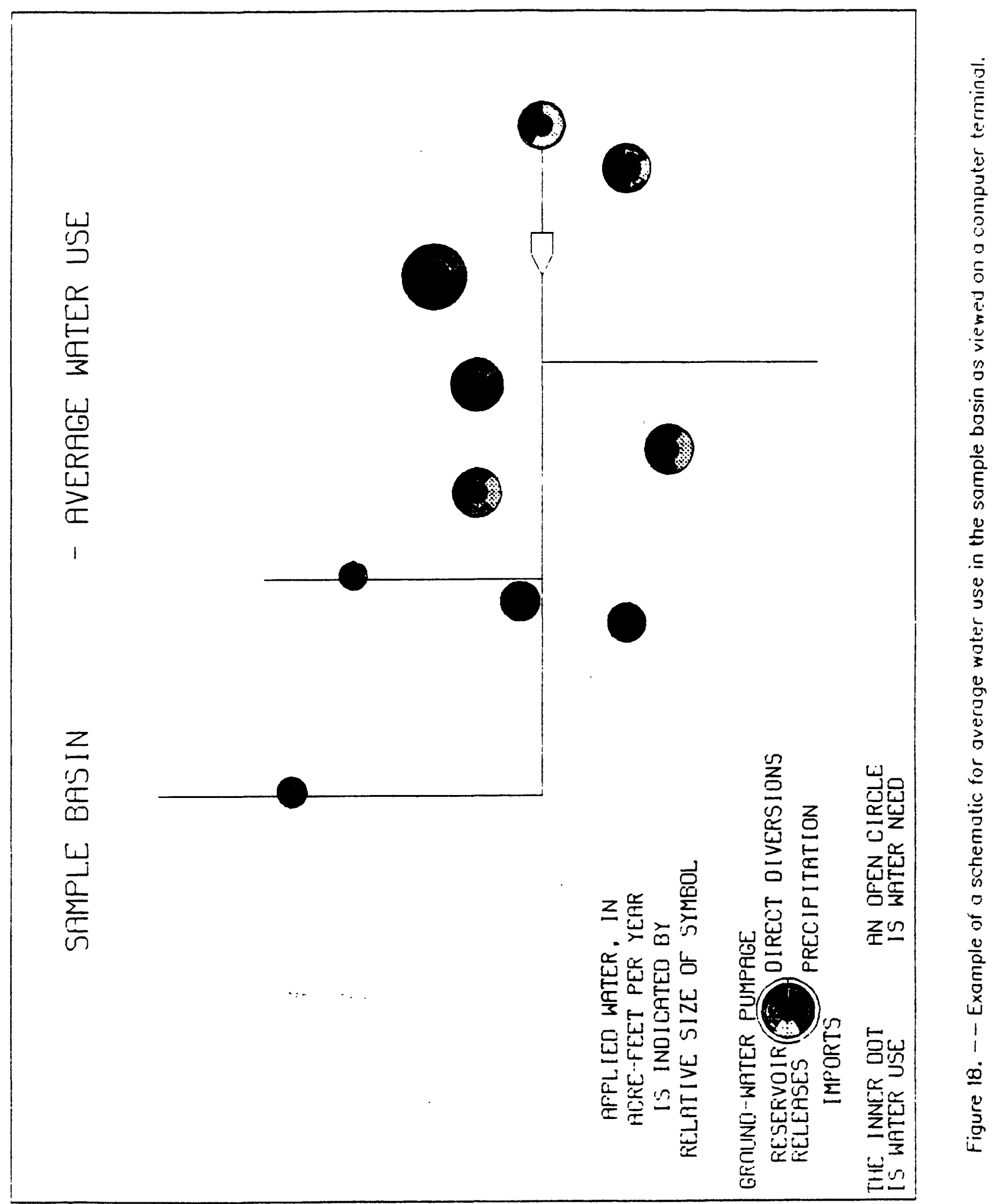




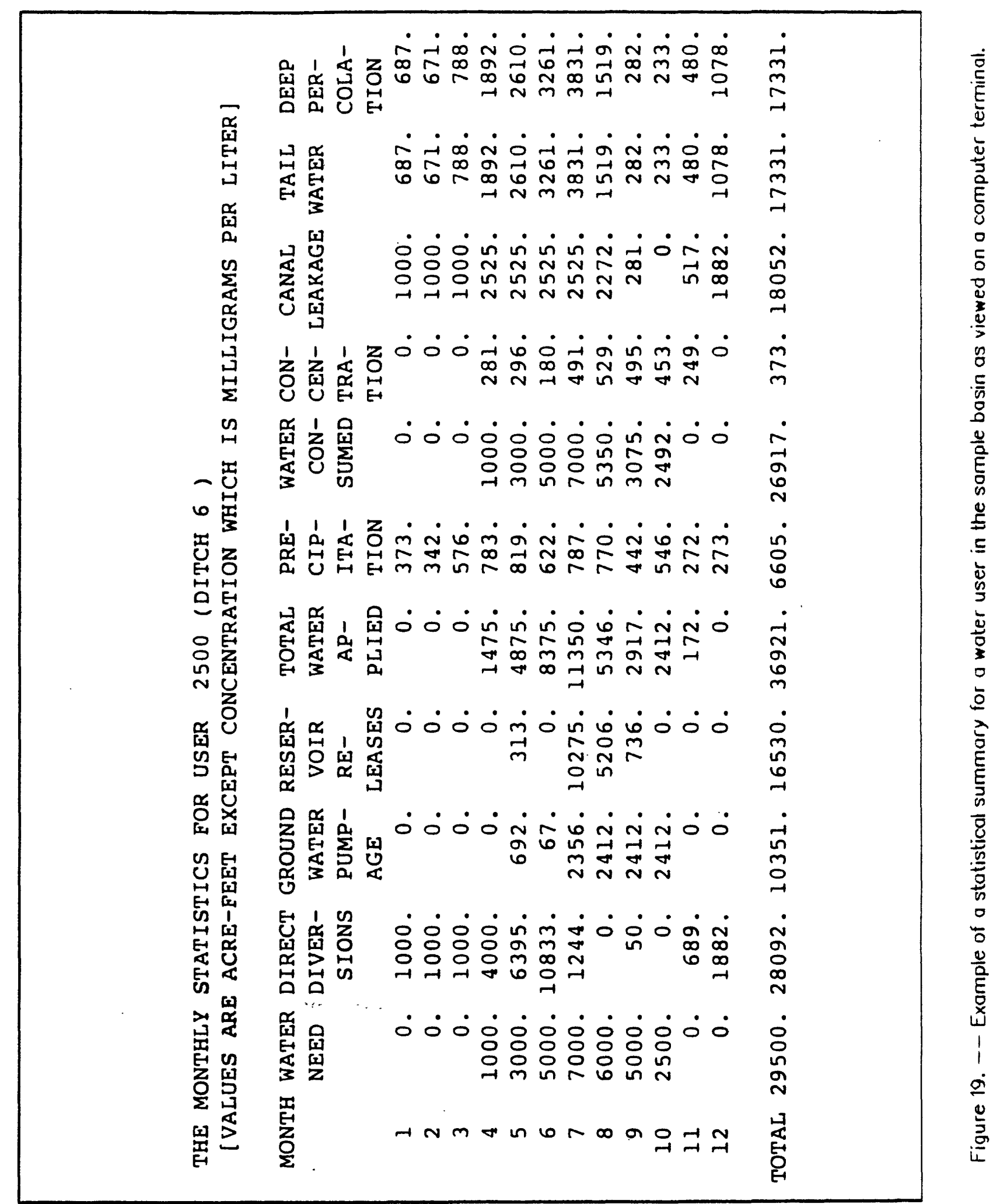




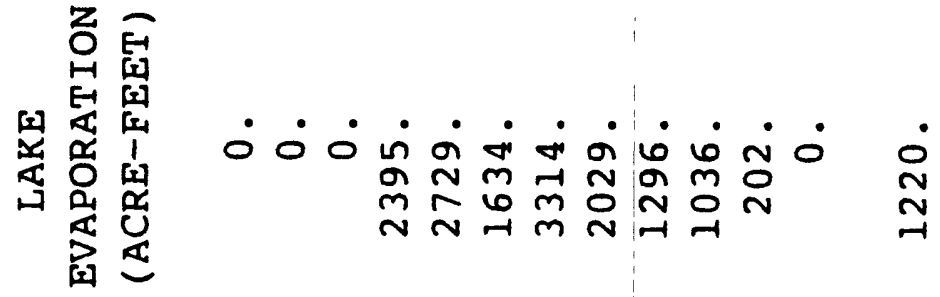

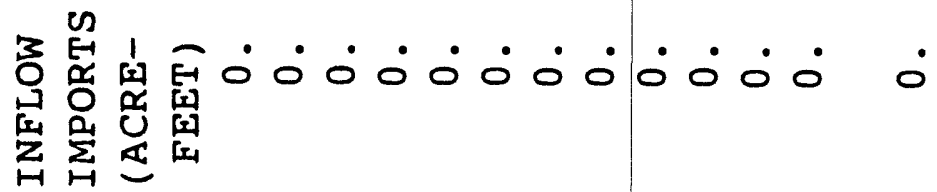

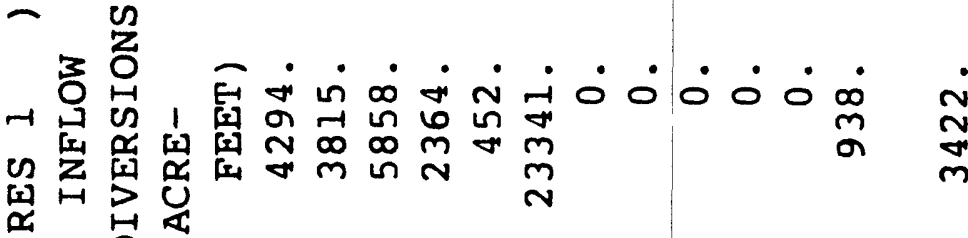

曲 H吕

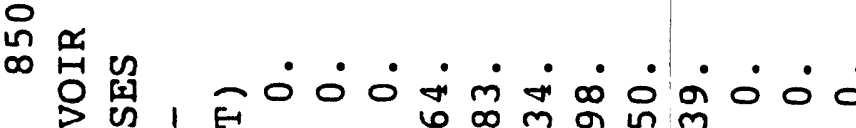

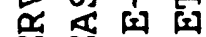

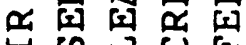

थै

의 ब저

只吃

บี

乙

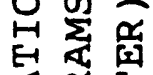

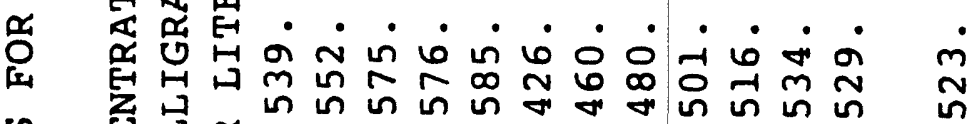

ध वै 号

U 号是罢

is $U$

E-1

E M E

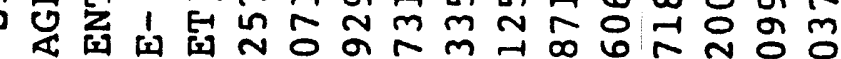

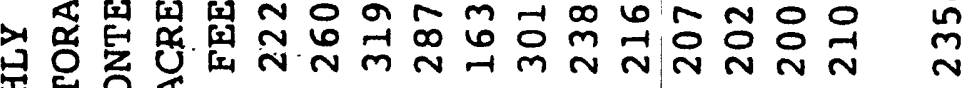

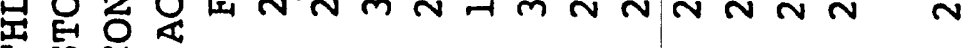

E in $U$ ป

焉

HNm $\infty \mathrm{n} \sim \infty$

a $\underset{f}{0} \underset{-1}{n}$

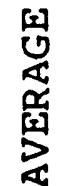


At the completion of a simulation, two final subroutines are available that provide comparative summaries of the just-completed simulation with one previously generated. CMPAR is the same as described for the streamflow simulation. Within CMPARU, the water need, direct diversions, ground-water pumpage, reservoir releases, imports, total application, or total water consumption of any water user can be compared with the same user and parameter from a previous simulation. Reservoir contents for any reservoir also can be compared. This comparison is done by reading the output file produced during the ongoing simulation (logical unit 52 ) and by reading some designated output from a previous simulation (logical unit 55). For any selected parameter, the subroutine computes the mean and standard deviation of the difference between the monthly values for the period of record, and displays three plots: (1) A histogram of both time series, similar to figure 9 ; (2) a frequency curve of each time series, similar to figure 10; and (3) a histogram of the time series of the difference between the two simulations, similar to figure 11 .

\section{SUMMARY}

An interactive-accounting model which simulates streamflow, water quality, and water-supply operations in a river basin was developed and described in this report. Regression equations are used to compute streamflow from a set of independent variables such as snowpack, precipitation, air temperature, or other streamflow. The concentrations of a conservative constituent, usually dissolved solids, also are computed with regression equations, with streamflow as the independent variable.

The model can be operated interactively, with the baseline data sets being modified to create new scenarios. Most of the model output can be displayed on a computer terminal interactively, in the form of colored graphics schematics, to show areal variations, and hydrographs, to show temporal variations.

The water-supply operations and a simplified ground-water system also can be simulated with the model. Various water users, such as agricultural, municipal, industrial, and in-stream users, plus reservoir operators are assigned water demands which they try to satisfy from an ordered list of water sources. Those sources may include direct diversions, ground-water pumpage, reservoir releases, or interbasin imports. All direct diversions are simulated such that the basinwide, prior appropriation priorities are recognized. 


\section{REFERENCES CITED}

Burns, A.W., 1983, Simulated hydrologic effects of possible ground-water and surface-water management alternatives in and near the Platte River, south-central Nebraska, in Hydrologic and geomorphic studies of the Platte River basin: U.S. Geological Survey Professional Paper 1277, p. G1-G30.

Fleming, D.E., 1975, The CORSIM project: Annual meeting of the American Society of Civil Engineers, November 4, 1975, Denver, 17 p.

Jenkins, C.T., 1968a, Computation of rate and volume of stream depletion by wells: U.S. Geological Survey Techniques of Water-Resources Investigations, Book 4, Chapter D1, 17 p.

$1968 \mathrm{~b}$, Techniques for computing rate and volume of stream depletion by wells: Ground Water, v. 6, no. 2, p. $37-46$. $1968 \mathrm{c}$, Electric-analog and digital-computer model analysis of stream depletion by wells: Ground Water, v. 6, no. 6, p. 27-34.

Parker, R.S. and Norris, J.M., 1983, Simulated effects of anticipated coal mining on dissolved solids in selected tributaries of the Yampa River, northwestern Colorado: U.S. Geological Survey Water-Resources Investigations Report 83-4084, 66 p.

Ribbens, R.W., 1975, Program NW01--River network program, User's manual: Denver, U.S. Bureau of Reclamation, Engineering and Research Center, Division of Planning Coordination, $140 \mathrm{p}$.

Shafer, J.M., 1979, An interactive river basin water management model-Synthesis and application: Colorado Water Resources Research Institute Technical Report 18, 244 p.

Taylor, 0.J. and Luckey, R.R., 1972, A new technique for estimating recharge using a digital model: Ground Water, v. 10, no. 6, 5 p. 1974, Water-management studies of a stream-aquifer system, Arkansas River valley, Colorado: Ground Water, v. 12 , no. 1,13 p.

Texas Water Development Board, 1974, Economic optimization and simulation techniques for management of regional water resource systems, river basin simulation model SIMYLD-II--Program description: Austin, $51 \mathrm{p}$.

U.S. Army Corps of Engineers, 1980, Computer program catalog of the Hydrologic Engineering Center: Davis, Calif., 16 p.

U.S. Army Engineer Division, North Pacific, 1972, Program description and user manual for SSARR model: Portland, Ore., $202 \mathrm{p}$.

U.S. Bureau of Reclamation, Upper Missouri Region, Division of Data Processing, 1978, HYDROSS-Hydrologic River Operation Study System, User's guide: Billings, Mont., 158 p. 


\section{ATTACHMENTS}

Attachment 1--Data Instructions For Streamflow and Water-Quality Simulation

Three data sets must be prepared prior to using the model for streamflow simulation:

A basin description of the modeled area (logical unit 60),

a digitized network of points along the river (logical unit 62), and

a time series of all the independent variables (logical unit 61).

Record Columns Name Description

The format for the basin-description file is:

$\begin{array}{rrl}1 & 1-80 & \text { TITLED } \\ 2 & 1-5 & \text { NNODE } \\ 3 & 1-10 & \text { QMAX } \\ & 11-20 & \text { QWL1 } \\ & 21-30 & \text { QWL2 } \\ & 31-40 & \text { QWL3 }\end{array}$

A title

The number of nodes (maximum of 100)

An estimate of the largest streamflow, in cubic feet per second (used for plotting purposes)

Value of water quality, in milligrams per liter, to subdivide class 1 from class 2

Value of water quality, in milligrams per liter, to subdivide class 2 from class 3

Value of water quality, in milligrams per liter, to subdivide class 3 from class 4

NOTE -- records 4 and 5 are entered as a set, one for each NNODE

$1-5$ NODEID

6- 13

NODNAM

$16-25$

$26-35$

YLOC

XIOC

36- 40 NXTNOD

$41-50$

$51-60$

$61-70$

PLTX

PLTY

CONCFC
A node ID number

A node name

Latitude, in decimal degrees

Longitude, in decimal degrees

Node ID of the downstream node. Must be set to negative number for all terminus nodes.

Plotting offset from the node symbol, in inches

Plotting offset from the node symbol, in inches

Concentration factor to determine load routing when incremental streamflow is negative. Value ranges from 0.0 to 1.0 , where 0.0 indicates the entire load is concentrated downstream and 1.0 indicates the concentration is maintained downstream.

71- 80 DSA Intercept of the water quality-specific conductance linear regression

81- 90 DSB Slope of the water quality-specific conductance linear regression

NOTE -- record 5 is repeated 12 times, one for each month, beginning with January.

5 11- 15 INDEPV Variable number of the independent variable.

If the value is positive, the incremental flow is computed and routed downstream.

If the value is negative, the total flow at that point is computed with no routing.

If the value is less than 100 , the flow is computed using linear regression and the independent variable identified by INDEPV. 
Attachment 1--Data Instructions For Streamflow and

Water-Quality Simulation--Continued

Record Columns Name Description

Format for the basin-description file--Continued

5 11- 15 INDEPV

If the value is greater than 100 , the flow is computed using log-log regression and the independent variable identified by (INDEPV - 100).

If the value is greater than 200 , the flow is computed using linear regression and the independent variable is upstream streamflow.

16- 25 NTRCPT Intercept of the streamflow-independent variable regression.

26- 35 SLOPE Slope of the streamflow-independent variable regression

36- 45 QWA Intercept of the specific conductance-streamflow log-log regression

46- 55 QWB Slope of the specific conductance-streamflow log-log regression

The format for the digitized network file is:

$1 \quad 1-10$ XMIN

11- 20 XMAX

21- 30 YMIN

$31-40$ YMAX

41- 50 XIENG

$51-60$ YLENG

$2 \quad 1-10 \quad \mathrm{XHY}$

$11-20$ YHY

$21-30 \quad \mathrm{XHY}$

$31-40$ YHY

41- $50 \quad \mathrm{XHY}$

$51-60 \quad Y H Y$

$61-70 \quad \mathrm{XHY}$

$71-80 \quad \mathrm{YHY}$

$81-90 \quad \mathrm{XHY}$

91-100 YHY
Largest longitude, in decimal degrees, for mapping Smallest longitude, in decimal degrees, for mapping Smallest latitude, in decimal degrees, for mapping Largest latitude, in decimal degrees, for mapping Length of $x$ axis, in decimal degrees, on schematic hydrographs

Length of $y$ axis, in decimal degrees, on schematic hydrographs

Longitude, in decimal degrees, to place first schematic hydrograph

Latitude, in decimal degrees, to place first schematic hydrograph

Longitude, in decimal degrees, to place second schematic hydrograph

Latitude, in decimal degrees, to place second schematic hydrograph

Longitude, in decimal degrees, to place third schematic hydrograph

Latitude, in decimal degrees, to place third schematic hydrograph

Longitude, in decimal degrees, to place fourth schematic hydrograph

Latitude, in decimal degrees, to place fourth schematic hydrograph

Longitude, in decimal degrees, to place fifth schematic hydrograph

Latitude, in decimal degrees, to place fifth schematic hydrograph 
Attachment 1--Data Instructions For Streamflow and

Water-Quality Simulation--Continued

\begin{tabular}{lll}
\hline Record Columns Name & Description \\
\hline
\end{tabular}

Format for the digitized network file--Continued

NOTE -- record 3 is repeated until ICARD is negative

3 1- 5 ICARD Code indicating disposition of data points.

If value is one, start plotting data in a new sequence.

If value is greater than 1 , continue plotting data in sequence from previous line.

If value is zero, skip the line.

If value is negative, quit plotting the network.

6- 12 YLINE Latitude, in decimal degrees, of a point

13- 19 XIINE Longitude, in decimal degrees, of a point

20- 26 YLINE Latitude, in decimal degrees, of a point

27- 33 XIINE Longitude, in decimal degrees, of a point

34- 40 YLINE Latitude, in decimal degrees, of a point

41-47 XIINE Longitude, in decimal degrees, of a point

48- 54 YLINE Latitude, in decimal degrees, of a point

55- 61 XIINE Longitude, in decimal degrees, of a point

62- 68 YIINE Latitude, in decimal degrees, of a point

69- 75 XIINE Longitude, in decimal degrees, of a point

The format for the independent variable file is:

$1 \quad 1-80$ TITLEP A title

2. 1- 5 NMONTH The number of months (maximum of 600)

6- 10 NINDV The number of independent variables (maximum of 75)

3 1- 6 VARNAM An independent variable name. There are NINDV

11- 16 VARNAM occurrences, eight per line.

21- 26 VARNAM

31- 36 VARNAM

41- 46 VARNAM

$51-56$ VARNAM

61- 66 VARNAM

71- 76 VARNAM

NOTE -- record 4 is reàd as a set, one for each NMONTH, beginning in a January

4 1- 10 VARIBL Value of the time series of independent variables.

11- 20 VARIBL There are NINDV occurrences, eight per line.

21- 30 VARIBL ( -99 . is used to indicate missing data).

$31-30$ VARIBL

41- 50 VARIBL

51- 60 VARIBL

61- 70 VARIBL

$71-80$ VARIBL 
$\stackrel{0}{\circ}$

$\stackrel{\circ}{-}$

$\stackrel{\circ}{\circ}$

$\stackrel{0}{0}$

ำ

$\stackrel{n}{?}$

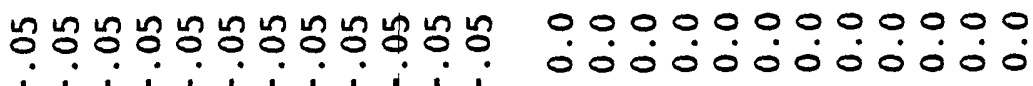

$\stackrel{?}{?}$

๕.

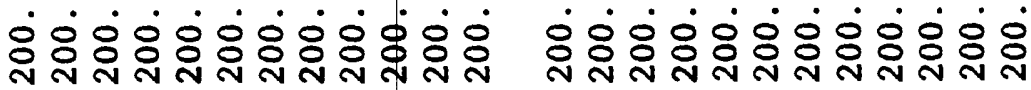
ठृ शू

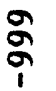

00000000000000000000000000

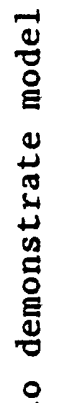

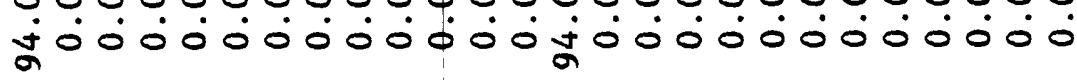
官

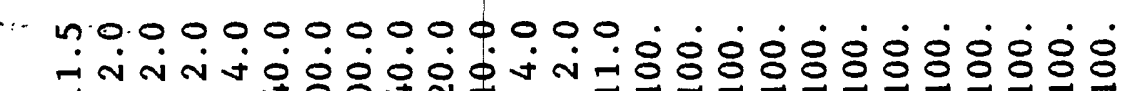
InN 음

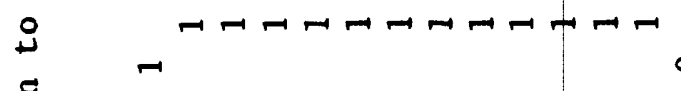
.

迢莒

$\sum_{0}^{2}$

总

농 
ำ

오

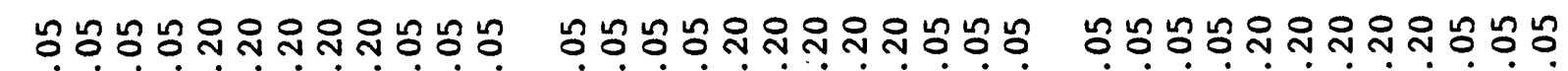
i 字 i

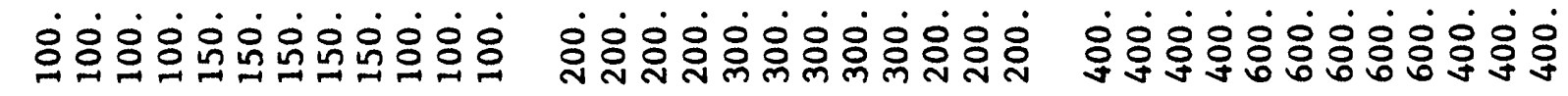
을 옹 8̊

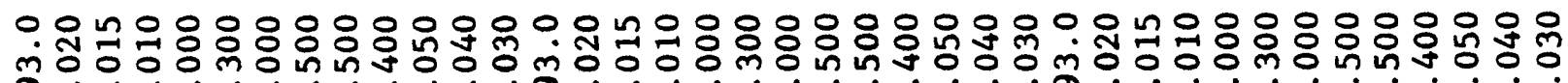
nूo.

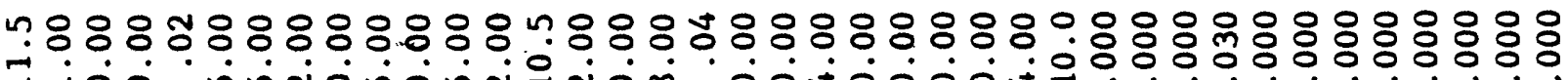

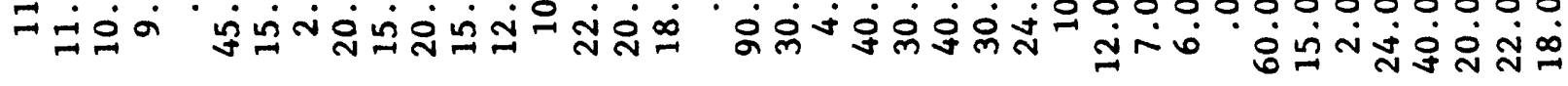

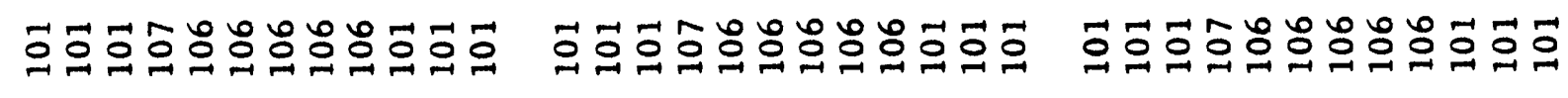
글 


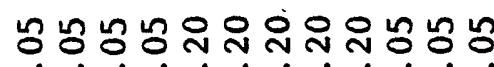

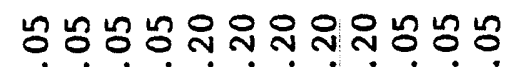

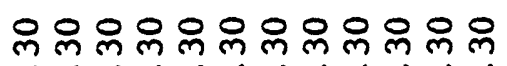
$\stackrel{8}{i}$ ? กิ

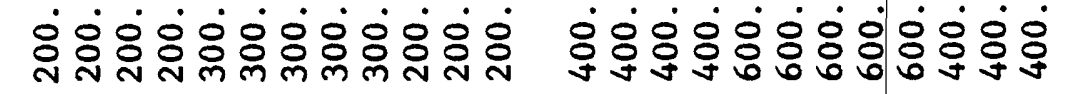
in \& (1)

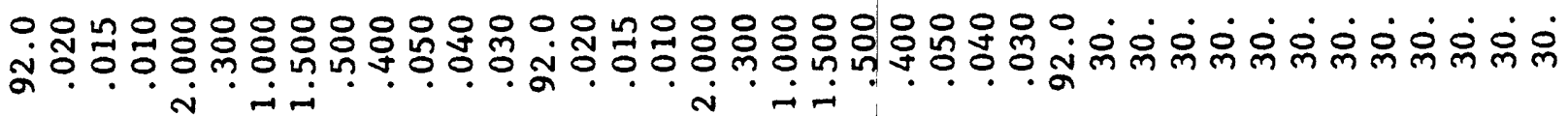

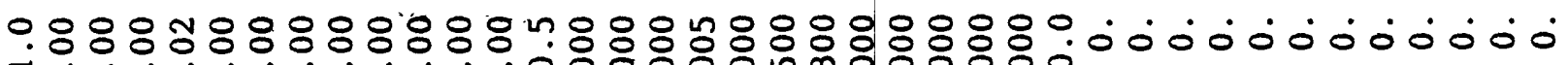
$=$ =

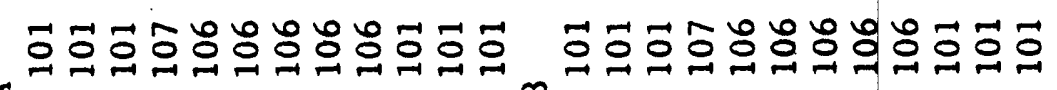
nNNNNNNNNNNN

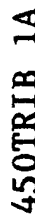
里 


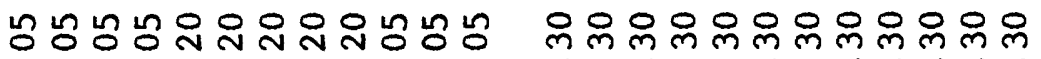

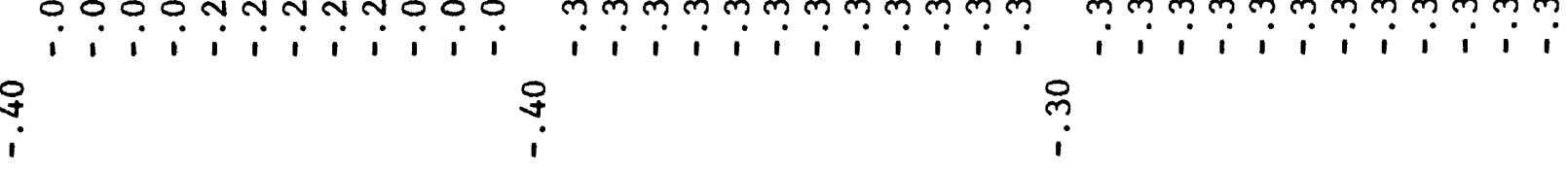

영ㅇㅇㅇㅇㅇㅇㅇㅇㅇㅇㅇㅇㅇㅇㅇ

$\stackrel{\circ}{\circ}$

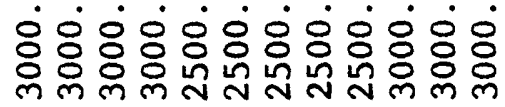
\&

ச் 芯

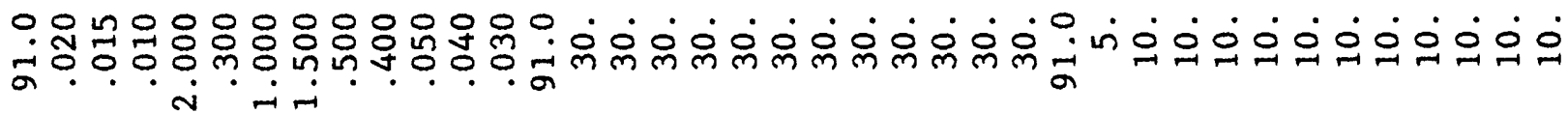

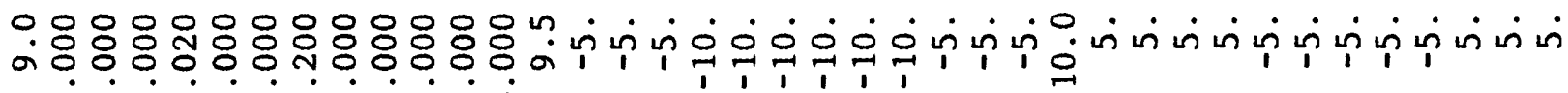

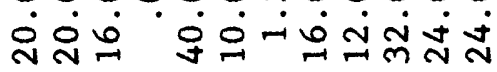

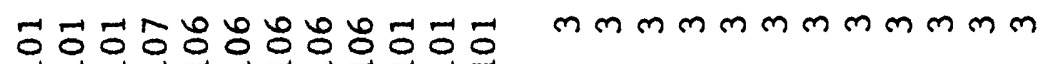

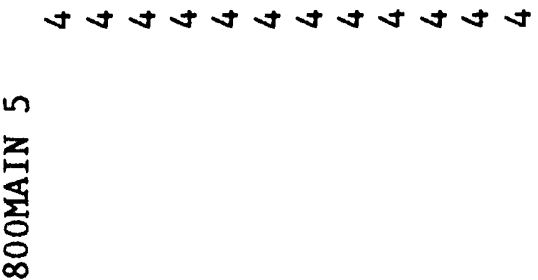


$\stackrel{\circ}{i} \quad \stackrel{\circ}{i}$

$\stackrel{\circ}{\circ}$

$\stackrel{\sim}{\circ}$

$\because+\dot{0}$

?

i. ?

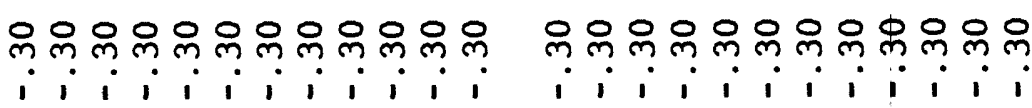

요

กิ

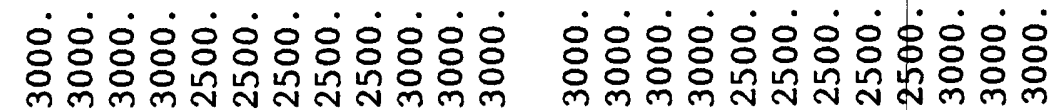
: \%े

$\stackrel{\circ}{\circ}$

$\stackrel{0}{0}$

웡ㅇㅇ용 응용으의 $\infty$

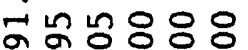
엉ㅇㅇㅇㅇ

๓

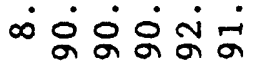

ผूํํㅇㅇㅇㅇㅇ 드응ㅇㅇㅇㅇ $\ddot{i} \dot{m}$

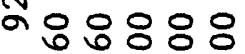
영ํํ

ㅇ..요용 $\sim^{\infty} \dot{0} \dot{0} \dot{0} \dot{0} \dot{0}$

n n 000000000 n n 0 n 000000000 n

Oे

ㅇํㅇㅇㅇㅛ

O n

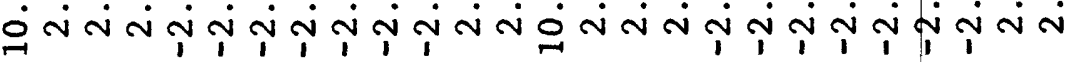

n

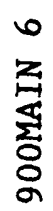

? $\infty \dot{\sigma}$

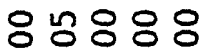
00000 no 성ㅇㅇ웡ㅇㅇㅇㅇㅇ

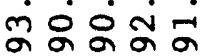
n $n^{\infty}$ 옹응 은 웅요

a ब $\rightarrow N m-T$ 
虽

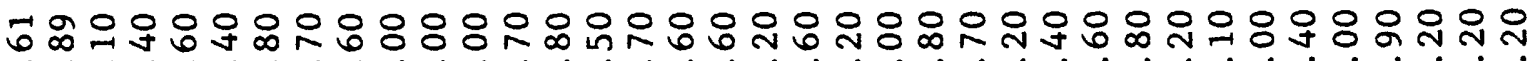

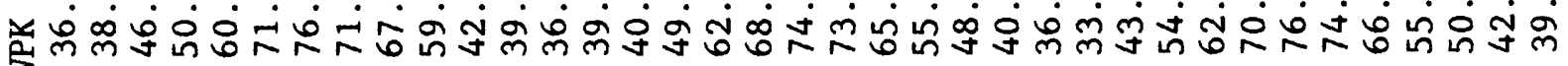
雚

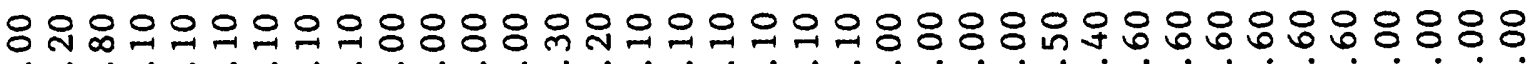

nğ́ zi

死

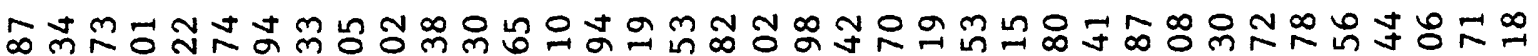

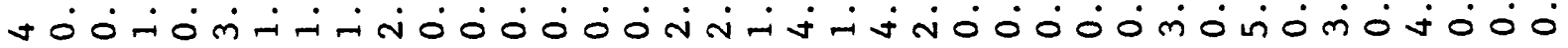
学

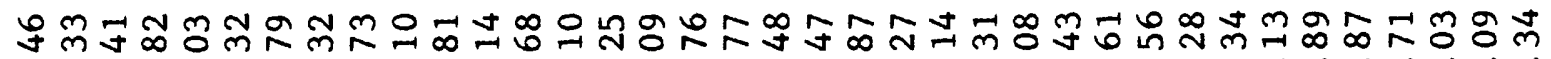

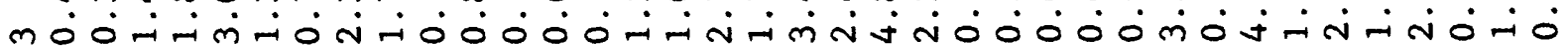
蛋

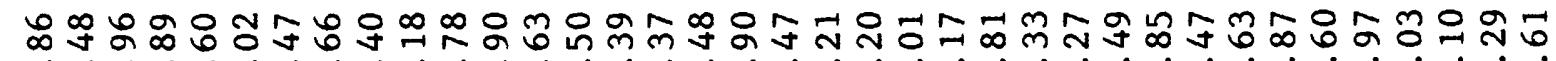
Nón-i 丞

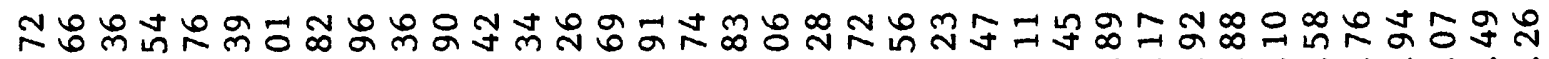

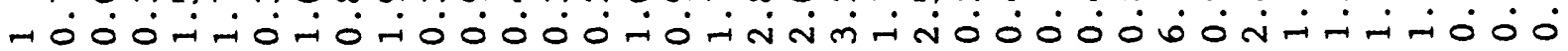
疍

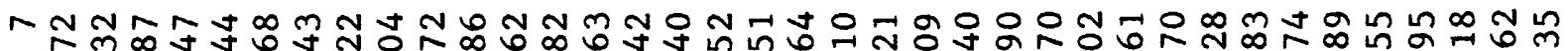

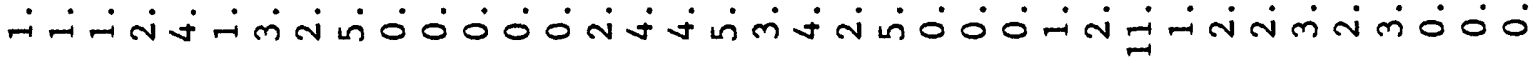
오 


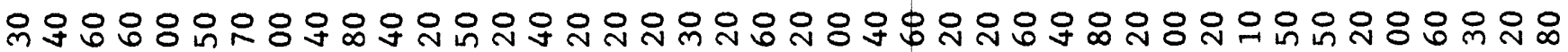

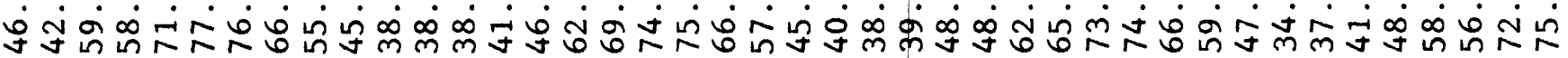

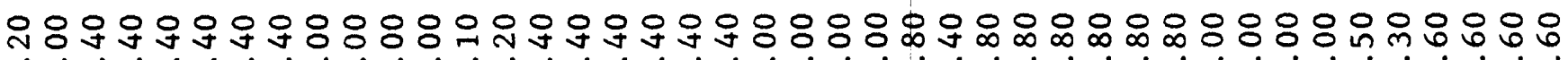

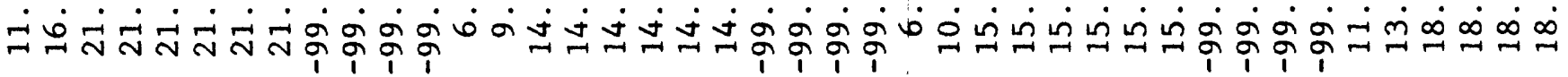

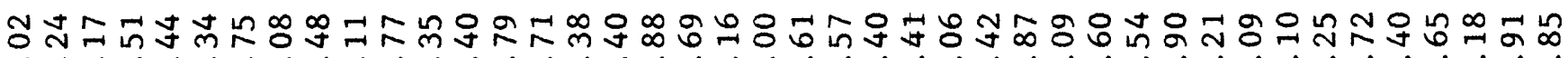

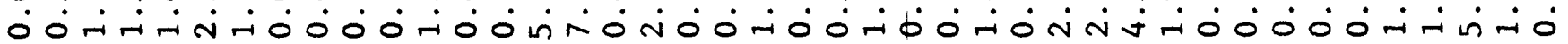

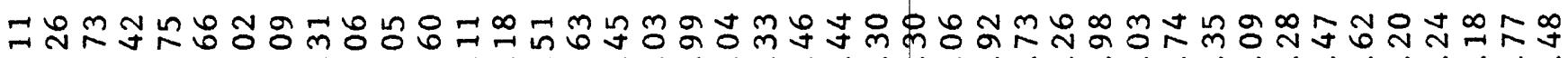

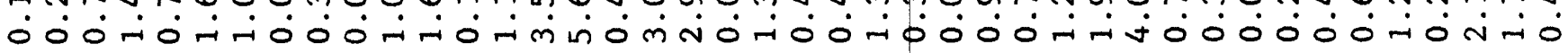

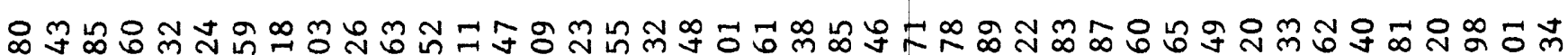

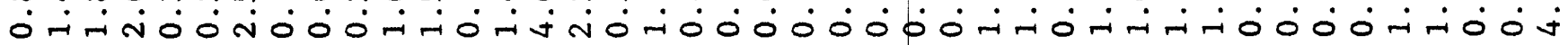

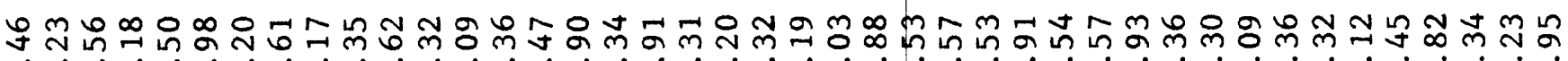

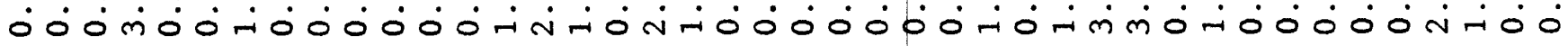

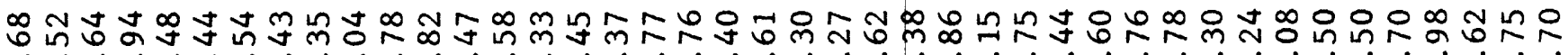

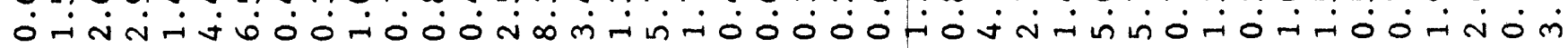




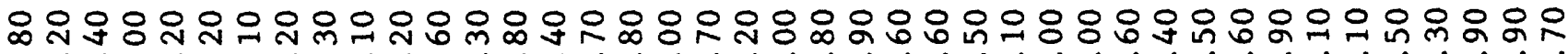

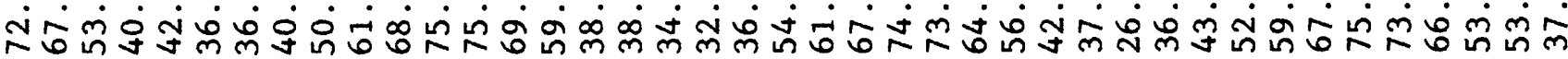

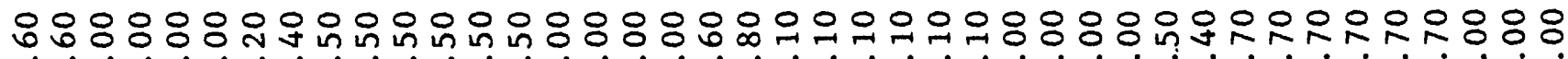

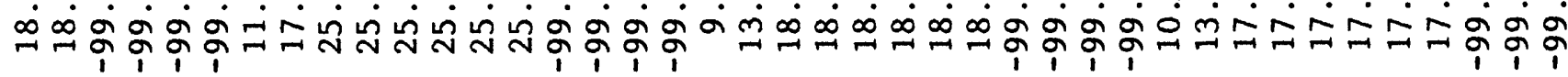

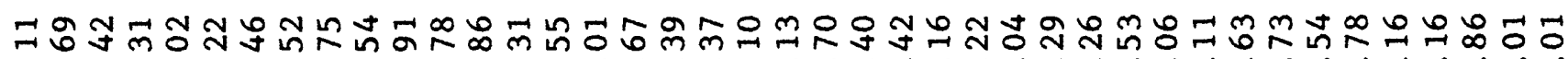

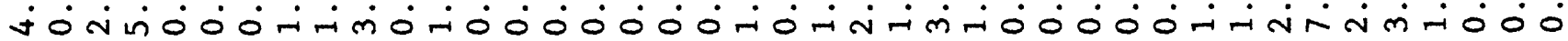

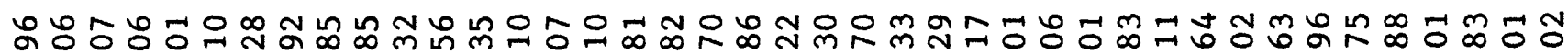

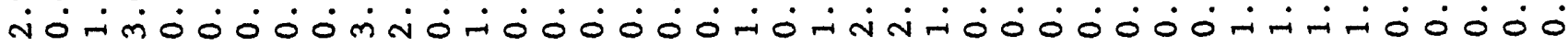

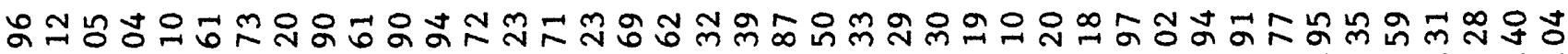

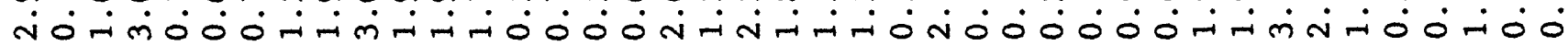

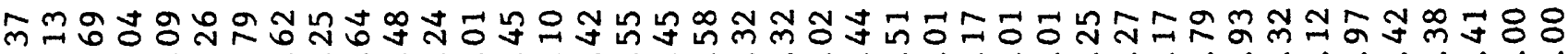

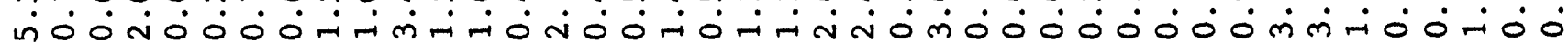

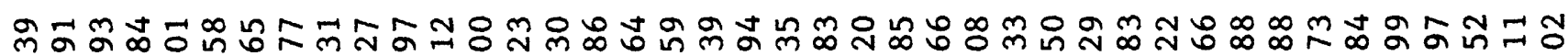
र́00 
Attachment 3--Data Instructions for Water-Supply Operations Simulation

Three additional data sets must be prepared prior to using the model for water-use simulation:

Additional basin descriptions of the modeled area (logical unit 53); a description of the water-supply operations (logical unit 50); and

a prioritized list of water rights (logical unit 51).

\begin{tabular}{lll}
\hline Record Columns Name Description \\
\hline
\end{tabular}

The format for the additional basin-description file is:

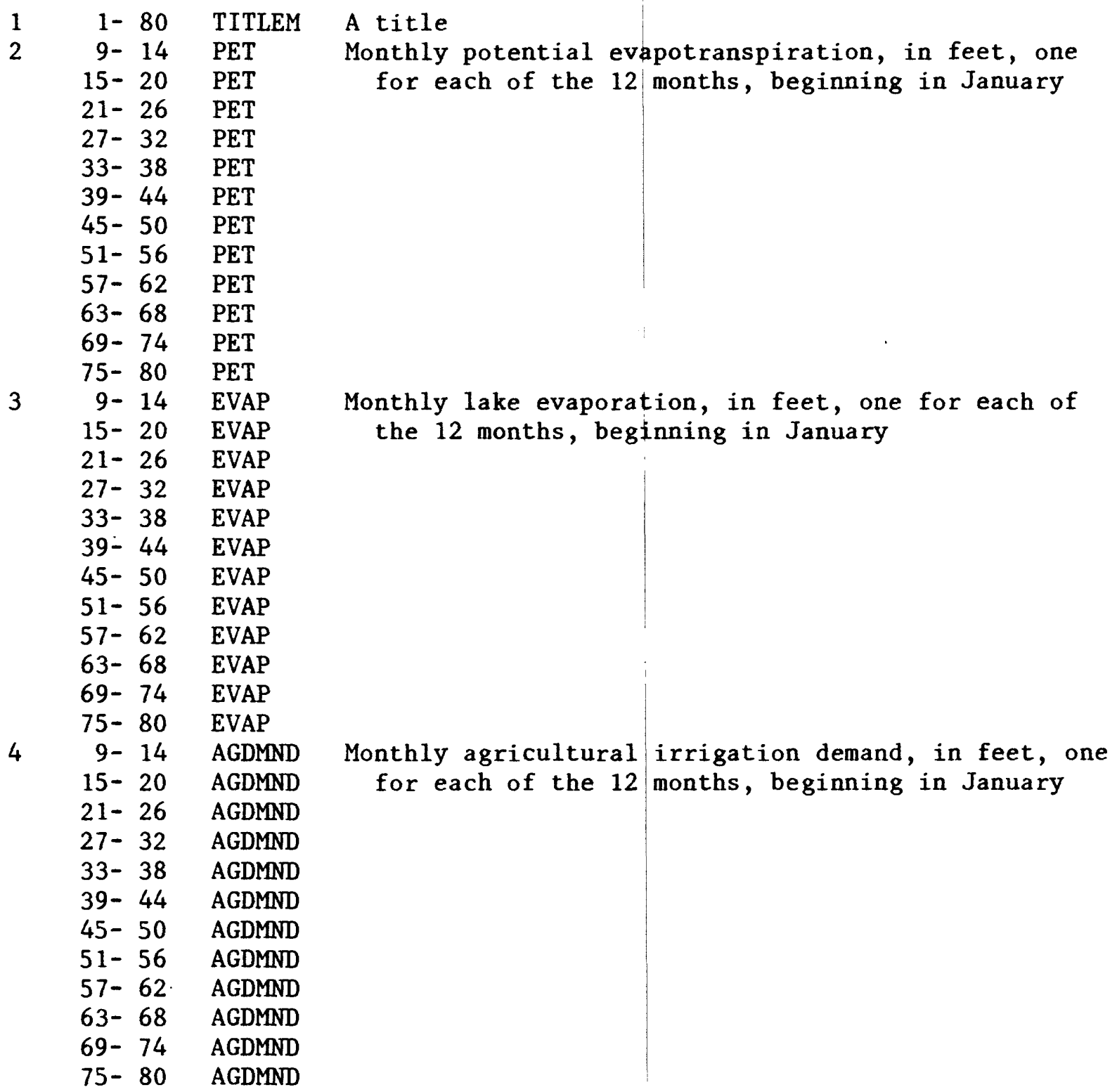


Attachment 3--Data Instructions for Water-Supply

Operations Simulation--Continued

\begin{tabular}{lll}
\hline Record Columns Name & Description \\
\hline
\end{tabular}

Format for the additional basin-description file--Continued

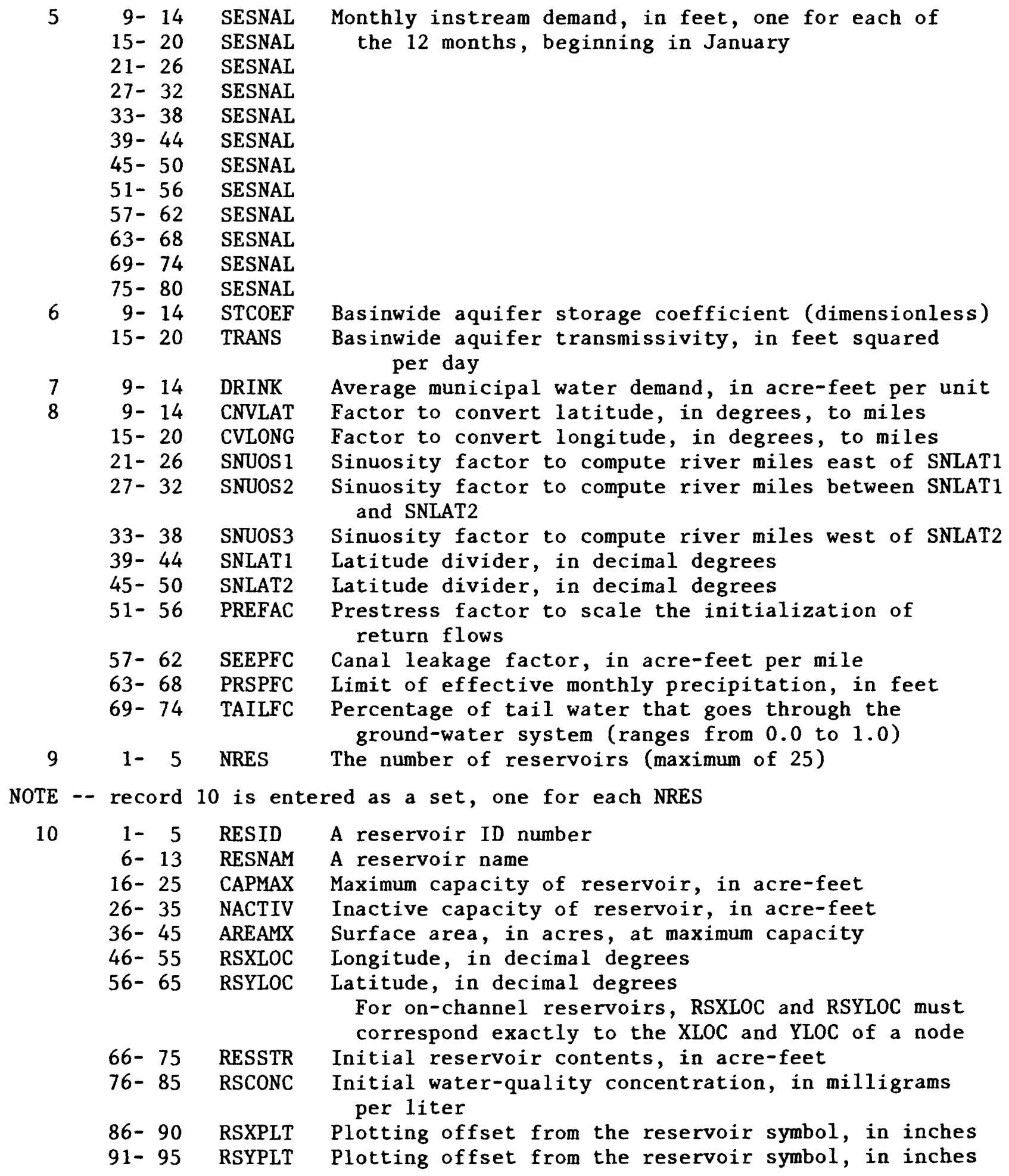


Attachment 3--Data Instructions for Water-Supply

Operations Simulation--Continued

\begin{tabular}{|c|c|c|c|c|}
\hline Record & Columns & Name & \multicolumn{2}{|c|}{ Description } \\
\hline \multirow{6}{*}{$\begin{array}{l}\text { Format } \\
\text { NOTE }\end{array}$} & for the & additiona & basin-description fil & e--Continued \\
\hline & record & 11 is ent & ced as a set, one for & each NNODE \\
\hline & $9-14$ & GWSTOR & $\begin{array}{l}\text { Initial ground-water } \\
\text { in acre-feet }\end{array}$ & storage above node on side 1 , \\
\hline & $15-20$ & GWCONC & $\begin{array}{l}\text { Initial water-quality } \\
\text { side } 1 \text {, in milligra }\end{array}$ & $\begin{array}{l}\text { concentration above node on } \\
\text { ms per liter }\end{array}$ \\
\hline & $21-26$ & GWSTOR & $\begin{array}{l}\text { Initial ground-water } \\
\text { in acre-feet }\end{array}$ & storage above node on side 2 , \\
\hline & $27-32$ & GWCONC & $\begin{array}{l}\text { Initial water-quality } \\
\text { side } 2 \text {, in milligra }\end{array}$ & $\begin{array}{l}\text { concentration above node on } \\
\text { ms per liter }\end{array}$ \\
\hline
\end{tabular}

The format for the water-supply operations file is:
$1 \quad 1-80$
TITLEU
A title
$2 \quad 1-5$ NUSERS
The number of water users (maximum of 100)

NOTE -- records $3,4,5$, and 6 are entered as a set, one for each NUSERS

$\begin{array}{rrr}1- & 5 & \text { USERID } \\ 6-13 & \text { USRNAM } \\ 16-20 & \text { USRTYP }\end{array}$

$21-30 \quad$ NUMUSR

31- 40 DMDFAC

41- $50 \quad$ XLOCU

$51-60 \quad$ YLOCU

$61-65$

$66-70$

$71-75$
A water user ID number
A water user name
The type of water user
1 = agricultural
2 = municipal
$3=$ industrial
$4=$ in stream
5 = reservoir operator

The quantity served by the water user

If type 1 , then the units are irrigated acres

If type 2, then the units are populace units

If type 3 , then the units are units produced

If type 4 , then the units are actual water needed, in acre-feet

If type 5, then the units are reservoir capacity available to the user, in acre-feet

actor to compute a water user's demand for water

If value is positive, multiply by PET,

If value is negative, multiply by AGDMND.

Longitude, in decimal degrees

Latitude, in decimal degrees

For reservoir operators, XLOCU and YLOCU must correspond exactly to the RSXIOC and RSYLOC of a reservoir USXPLT Plotting offset from the water user symbol, in inches
USYPLT Plotting offset from the water user symbol, in inches IPRECP Number indicating which of the independent variables represents the appropriate precipitation data for the water user. 
Attachment 3--Data Instructions for Water-Supply

Operations Simulation--Continued

Record Columns Name Description

Format for the water-supply operations file--Continued

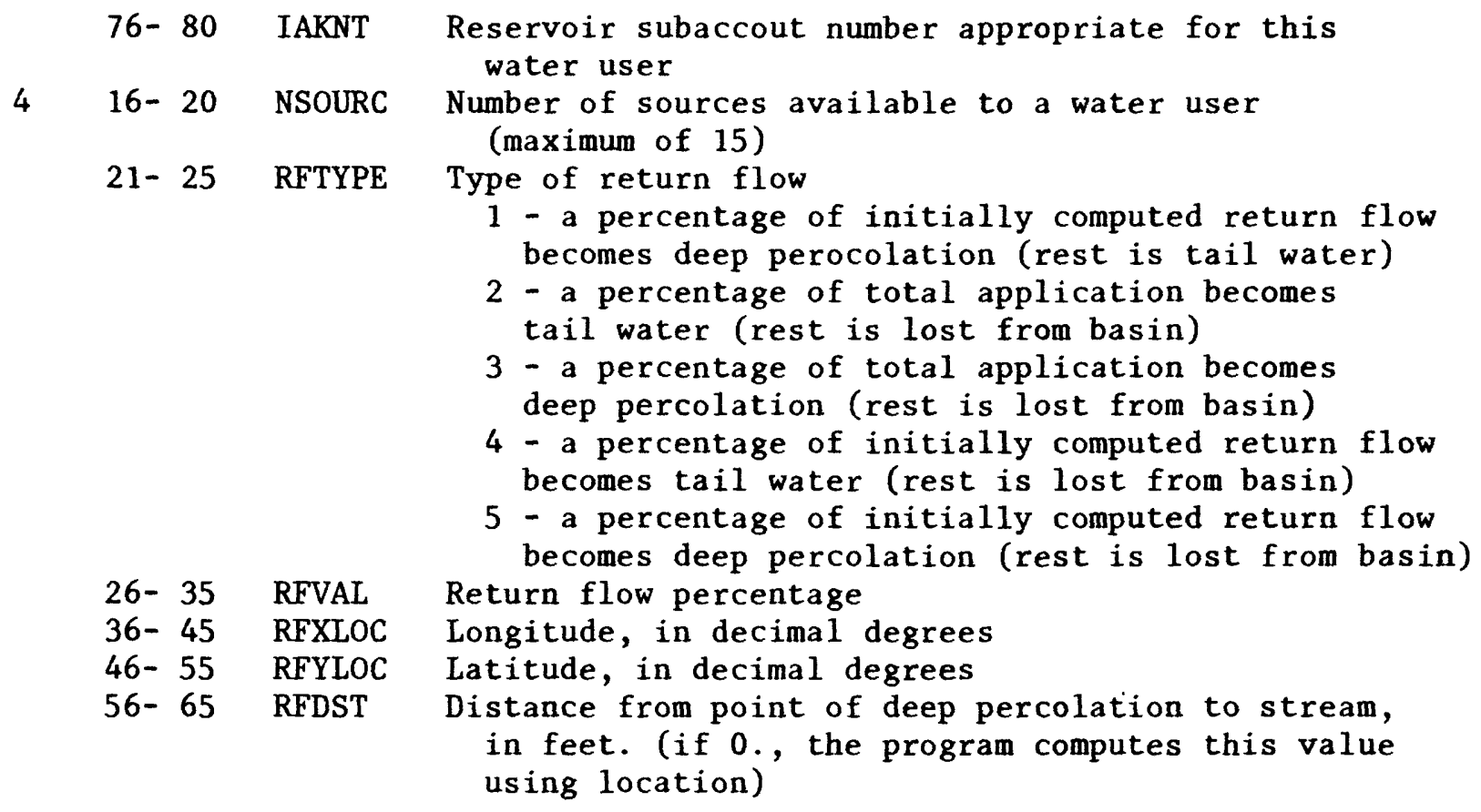

NOTE -- record 5 is repeated NSOURC times

5 1- 5 ICODE Type of water source

1 = direct diversion

2 = ground-water pumpage

3 = reservoir release (if reservoir releases directly to the water user rather than using the river as a conduit, enter -3 )

4 = direct pipeline or tunnel importation

5 = storage diversion

6 = direct diversion used for reservoir storage

6- 15 QUANTY The quantity available from the source

If type 1, then the legal right, in cubic feet per second

If type 2, then the pumping capacity, in cubic feet per second

If type 3, then the capacity of the reservoir allocated to this water user, in acre-feet (if the reservoir is a 'general-use', 'bankaccount' type storage, enter the percentage allocated to this user as a negative)

If type 4, then the pipeline or tunnel carrying capacity, in acre-feet

If type 5, then the legal right, in acre-feet

If type 6 , then the legal right, in cubic feet per second 
Attachment 3--Data Instructions for Water-Supply

Operations Simulation--Continued

\begin{tabular}{lll}
\hline Record Columns Name & Description \\
\hline
\end{tabular}

Format for the water-supply operations file--Continued

16- 25 SCXLOC Longitude, in decimal degrees

26- 35 SCYLOC Latitude, in decimal degrees

36- 45 GWDST The distance from point of pumpage to stream, in feet.

If 0. , the program computes this value using location.

46- 50 NMRES The ID number of the appropriate reservoir (RESID)

51-55 IACCNT Subaccount number to identify portion of a reservoir

allocated to this water user (if 0 , set to 1 )

56- 60 ITIMFC Code to indicate whether the source is available every month (if 0 , it is; if 1 , read TIMFAC)

NOTE -- record 6 is read only when ITIMFC is nonzero

6 6- 10 TIMFAC Percentage indicating how much of the source is

11- 15 TIMFAC active in each of the 12 months, beginning

16- 20 TIMFAC in January. A value of 1 . indicates fully

21- 25 TIMFAC active, a value of 0 . indicates the source is

26- 30 TIMFAC inactive during that particular month.

31- 35 TIMFAC

36- 40 TIMFAC

41- 45 TIMFAC

46- 50 TIMFAC

$51-55$ TIMFAC

$56-60$ TIMFAC

61- 65 TIMFAC

The format for the water rights file is:

$1 \quad 1-80$ TITLER A title

2 1- 5 NRIGHT The number of water rights (maximum of 200)

NOTE -- record 3 is repeated NRIGHT times

3 1- 5 OWNER The water user ID (USERID) who owns the right

6- 13 NAME The water user name (USRNAM) who owns the right

16- 23 DATE

24- 33 AMNT The amount of the water right (QUANTY), in cubic feet per second for direct diversions, and in acre-feet for storage diversions.

NOTE -- all of the record 3's must be ordered by DATE. 
$\stackrel{\text { ñ }}{0}$

는으우

을옹든

웅

옹으웅 ○0ं0

유ㅇㅠㅛ

ㄴํㄴ융요웅

O000 T No

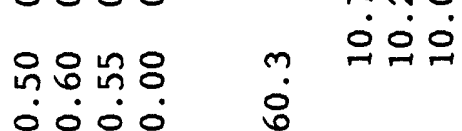

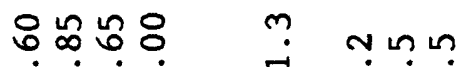
000 नूनूठ

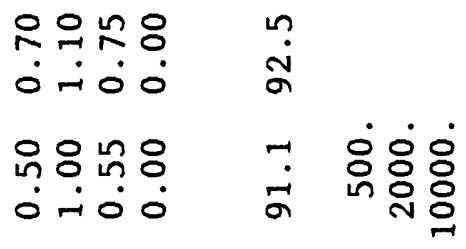
융ํㅇㅇㅛ 눙

os

की की

.

.

क्ष 3

प⿺

هั

c

in 07

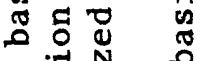

-

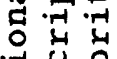

in 0

每 告

选 0

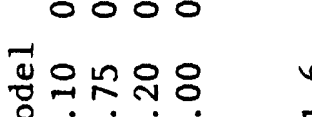

엉ㅇ

- $\dot{0} 0 \dot{0}$

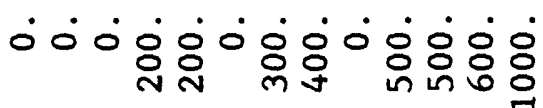

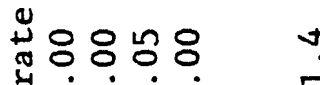

出0000

$\stackrel{\circ}{+}$

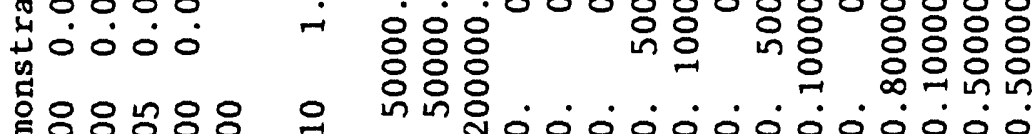

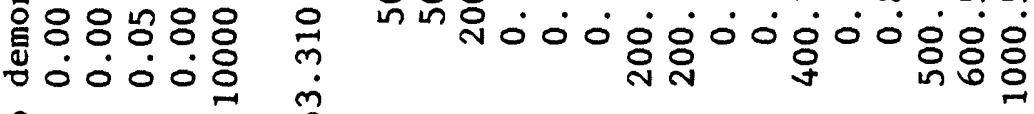
용ㅇㅇㅇㅇㅇำ 击00000 مـ

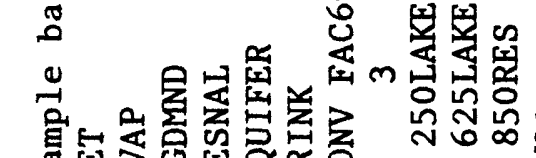

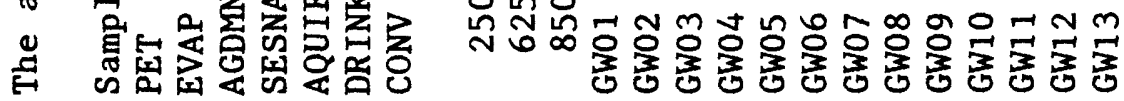



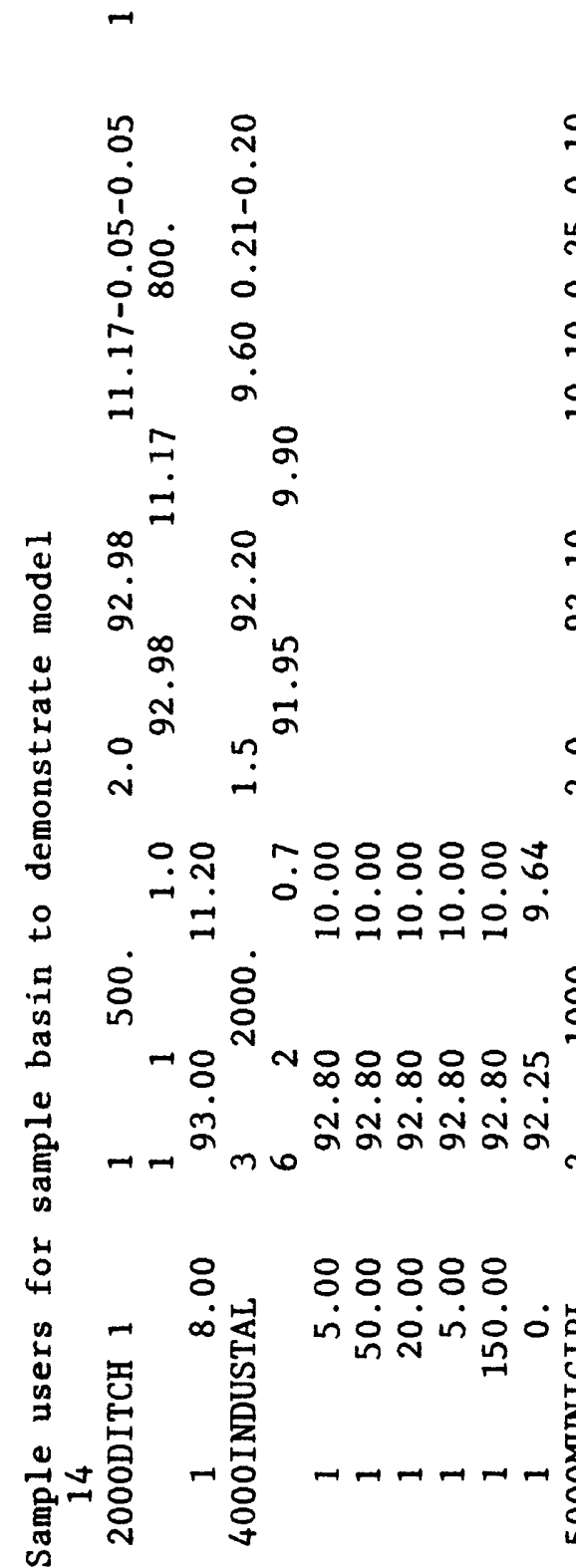

$\stackrel{0}{\circ}$

กิ

워

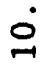

$\stackrel{n}{0}$

สู

$\dot{\sim}^{\stackrel{2}{\circ}}$

$\stackrel{0}{\circ} \dot{0}$

แ่

:융

b

$\stackrel{\infty}{\circ}$

ஓं

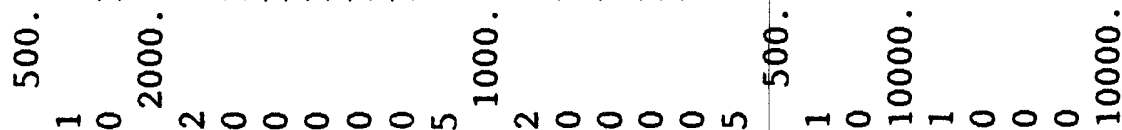

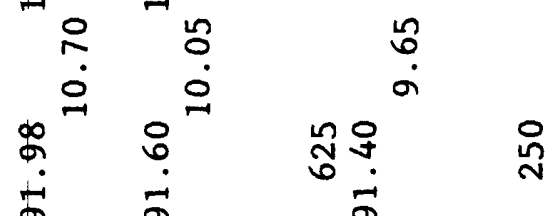

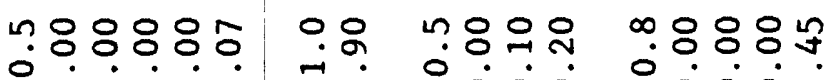
웅ㅇㅇㅇ

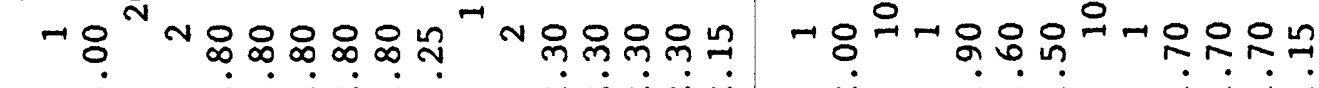
- ๙̆ं

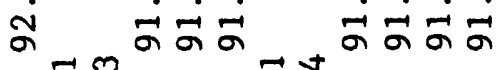

: $888: 8:$

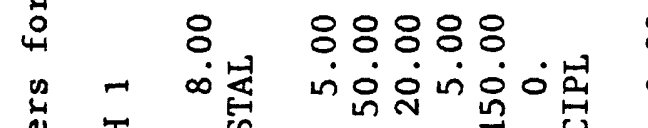


m $\quad$ n

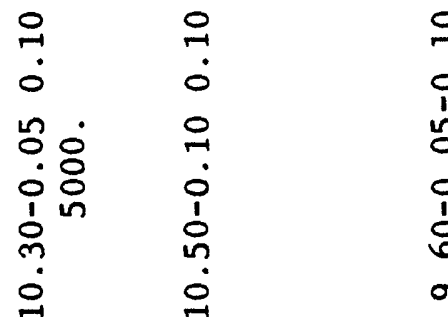

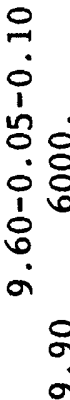

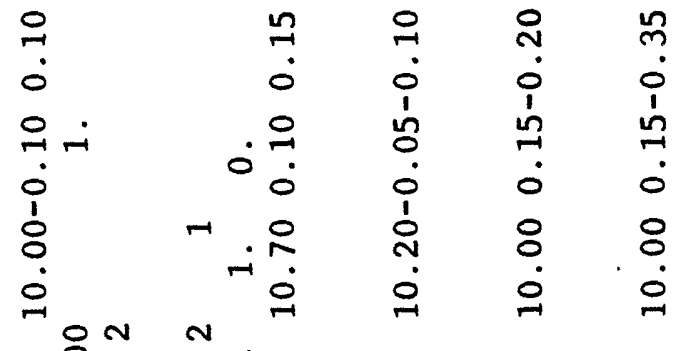

$\stackrel{0}{0} \quad \stackrel{0}{0}$

o

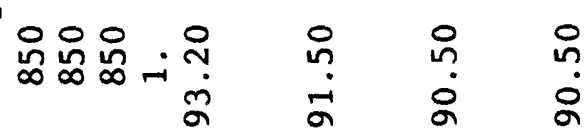

용ㅇㅇㅇ

in

$\overrightarrow{1}$

$\dot{-}$

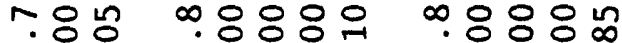
0 $\dot{0} 00 \dot{0} 0 \dot{0} \dot{0} 0$

0.

$08: 80$

$\begin{array}{cccc}0 & 0 & -1 & 0 \\ -i & \dot{1} & \dot{0} & \dot{H}\end{array}$

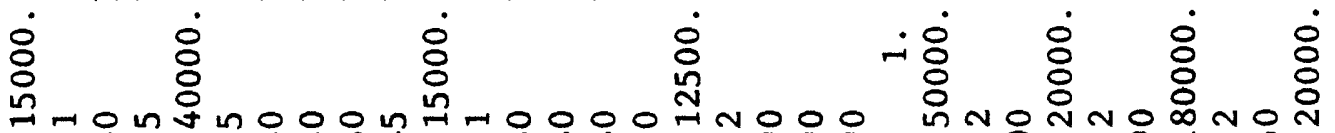

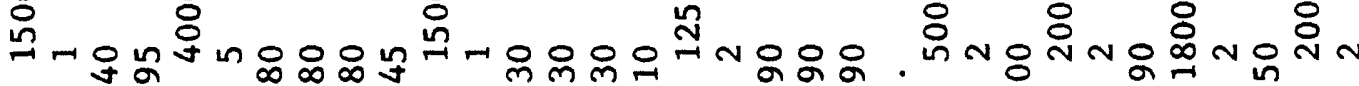

ने व

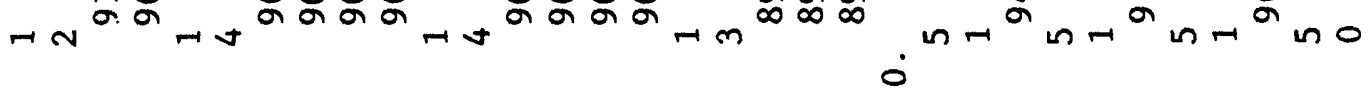

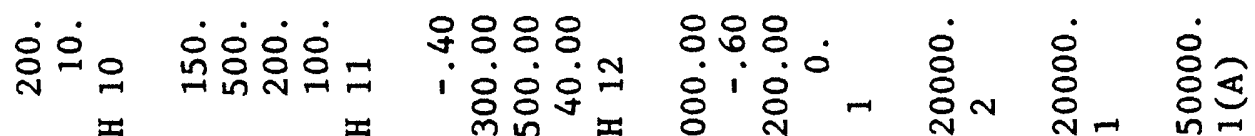

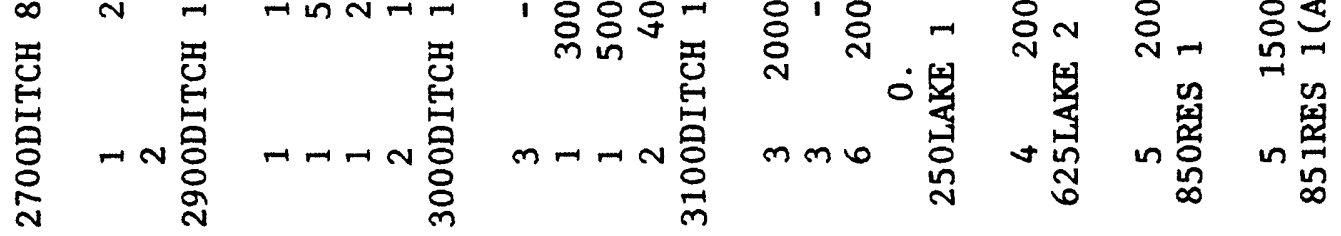




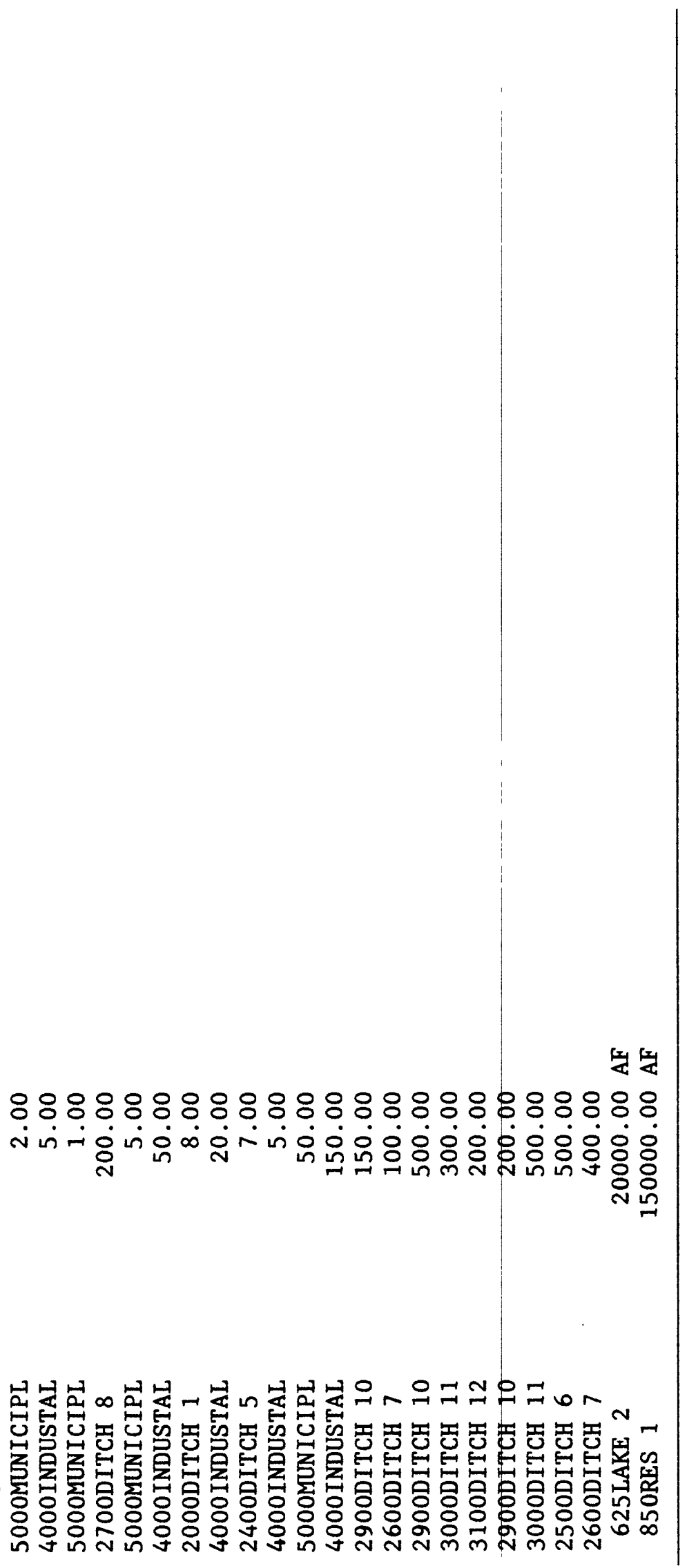


Attachment 5--Definition of Commoned Variables in Subroutines for Streamflow and Water-Quality Simulation

[Variables preceeded by $a *$ are read by the model]

\section{UNLABELED COMMON}

$\begin{array}{ll}\text { *IBATCH } & \text { - Indicator of whether job is being run interactive } \\ & \text { or batch. } \\ \text { *IGRAPH } & \text { - Code for type of graphics terminal. } \\ \text { IMONTH } & \text { - Counter for the month number. } \\ \text { IN } & \text { - Logical unit number for input. } \\ \text { IOUT } & \text { - Logical unit number for output. } \\ * \text { FIPRINT } & \text { - Indicator of whether monthly output should be } \\ & \text { printed interactively. } \\ \text { *IQW } & \text { - Indicator of whether to use time-weighted or } \\ & \text { discharge-weighted water-quality statistics. } \\ \text { IYEAR } & \text { - Counter for the year number. } \\ \text { JMONTH } & \text { - Counter for the month of the year. } \\ \text { *NINDV } & \text { - Number of independent variables in input file. } \\ \text { *NMONTH } & \text { - Number of months of independent variables in } \\ & \text { input file. }\end{array}$

\section{HYDROG}

*IHY (5)

QHY $(400,5)$

QWHY $(400,5)$

QWLHY $(400,5)$

- Node ID of those nodes for which hydrograph will be plotted.

- Time series of stream discharge to be plotted.

- Time series of water-quality concentration to be plotted.

- Time series of water-quality load to be plotted.

\section{NAMES}

$*$ TITL

- Title of simulation.

\section{NTWRK}

$* X H Y(5)$

XINC

*XIENG

*XMAX

*XMIN

$\star$ YHY $(5)$

YINC

*YLENG

*YMAX

*YMIN
- X-coordinate for the location of small hydrograph on the schematic.

- X-axis tick mark increment on schematic.

- Horizontal length of small hydrograph on schematic.

- Maximum $x$-coordinate for edge of schematic.

- Minimum $x$-coordinate for edge of schematic.

- Y-coordinate for the location of small hydrograph on the schematic.

- Y-axis tick mark increment on schematic.

- Vertical length of small hydrograph on schematic.

- Maximum y-coordinate for edge of schematic.

- Minimum $y$-coordinate for edge of schematic. 
Attachment 5--Definition of Commoned Variables in Subroutines for

Streamflow and Water-Quality Simulation--Continued

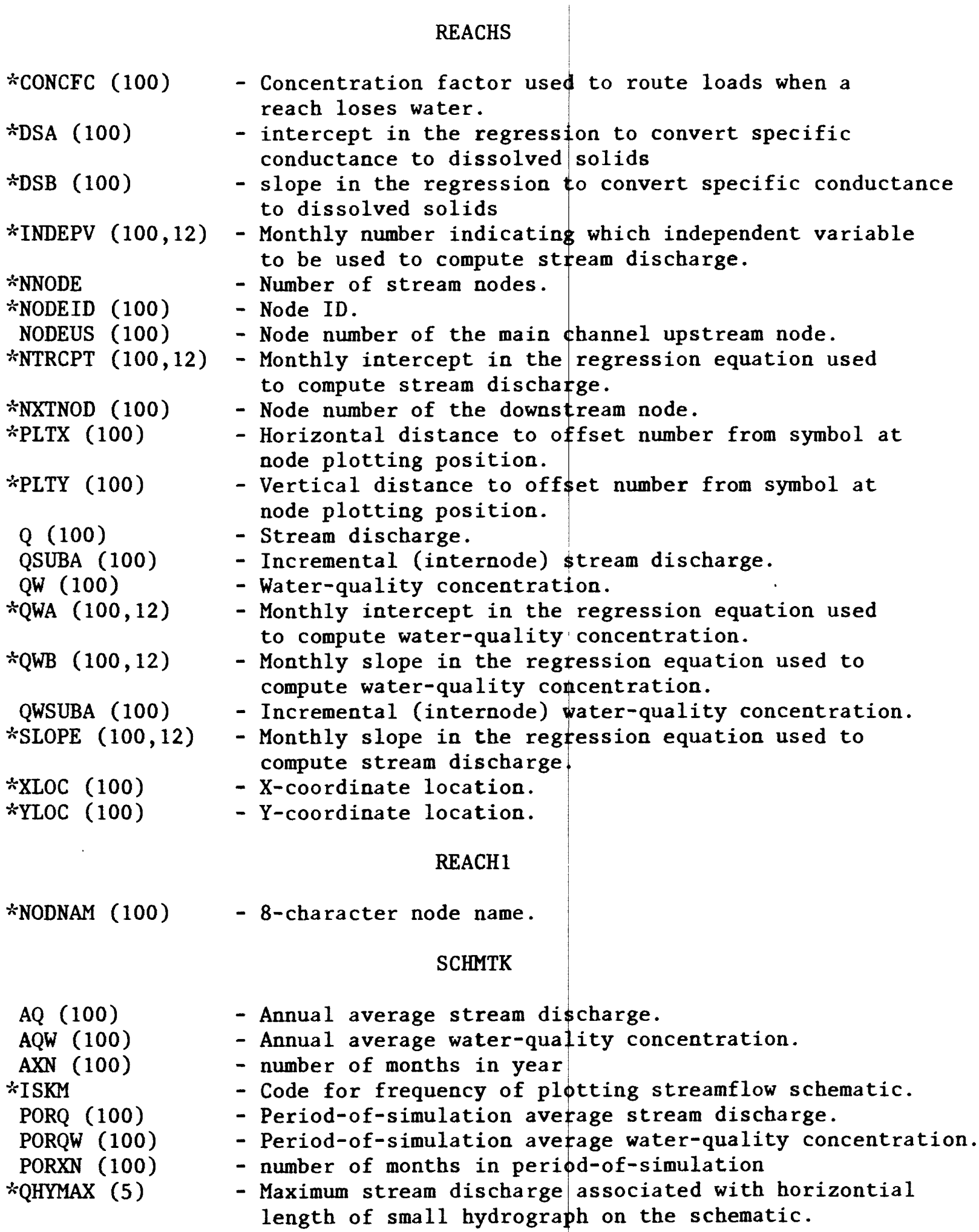


Attachment 5--Definition of Commoned Variables in Subroutines for Streamflow and Water-Quality Simulation--Continued

SCLFAC

$\begin{array}{ll}* \text { QMAX } & \text { - Maximum stream discharge associated with symbol size } \\ & \text { on the schematic } \\ * \text { QWL1 } & \text { - Water-quality concentration class limit. } \\ * \text { QWL2 } & \text { - Water-quality concentration class limit. } \\ * \text { QWL3 } & \text { - Water-quality concentration class limit. } \\ & \text { STATS } \\ * \text { ISS }(10) & \text { - Node ID of those nodes for which statistical } \\ \text { QMEAN }(10,12) & \text { - Monthly mean stream dsicharge. } \\ \text { QSD }(10,12) & \text { - Monthly standard deviation of stream discharge. } \\ \text { QWLMN }(10,12) & \text { - Monthly mean water-quality load. } \\ \text { QWLSD }(10,12) & \text { - Monthly standard deviation of water-quality load. } \\ \text { QWMEAN }(10,12) & \text { - Monthly mean water-quality concentration. } \\ \text { QWSD }(10,12) & \text { - Monthly standard deviation of water-quality } \\ \text { XNN }(10,12) & \text { - Numcentration. } \\ & \end{array}$ 
Attachment 6--Definition of Commoned Variables in Subroutines for Water-Supply Operations Simulation

[Variables preceeded by $a *$ are read by the model]

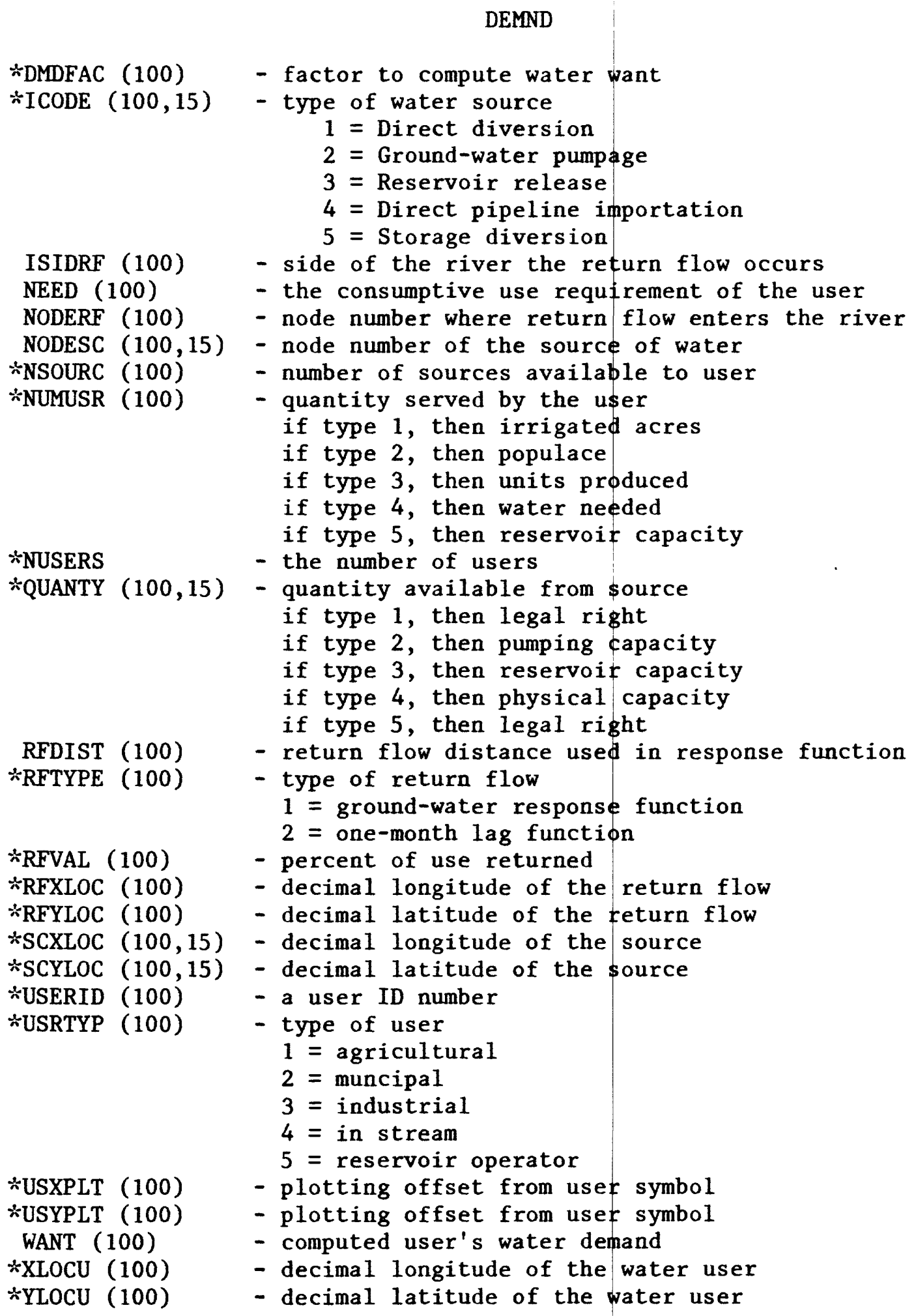


Attachment 6--Definition of Commoned Variables in Subroutines for Water-Supply Operations Simulation--Continued

DEMND 1

*USRNAM (100) - an 8 character user name

GW

*GWCONC (100) - concentration of ground water in node reach

$\star$ GWSTOR (100) - quantity of water in ground-water storage in node reach

RTRNFL (100) - quantity of ground-water entering river that month in node reach

GWUSE

\begin{tabular}{|c|c|}
\hline GWDIST (100) & umpage distance used in response $f$ \\
\hline GWPUMP (100) & the pumpage \\
\hline GWSOUR (100) & - source number of the pumpage source \\
\hline GWUSER (100) & - user number of the pumpage source \\
\hline ISDPMP $(100)$ & - side of the river the pumpage occurs \\
\hline MGW & - number of pumpage sources \\
\hline
\end{tabular}

HYDGRG

GWHYC $(500,5)$ - time series of ground-water concentration to be plotted

GWHYRF $(500,5)$ - time series of ground-water return flows to be plotted

GWHYS $(500,5)$ - time series of ground-water storage to be plotted

$*$ IHYGW (5) - code for node ID and side for which hydrographs will be plotted

\section{HYDGRR}

*IHYR (5)

- reservoir ID of those reservoirs for which hydrographs will be plotted

RHYC $(500,5)$

- time series of reservoir concentration to be plotted

RHYS $(500,5)$

- time series of reservoir storage contents to be plotted

HYDGRU

$\begin{array}{ll}* \text { IHYU (5) } & \text { - water user ID of those water users for which hydrographs } \\ & \text { will be plotted } \\ \text { UHYD }(400,5) & \text { - time series of direct diversions to be plotted } \\ \text { UHYP }(400,5) & \text { - time series of pumpage to be plotted } \\ \text { UHYT }(400,5) & \text { - time series of total applications to be plotted }\end{array}$


Attachment 6--Definition of Commoned Variables in Subroutines for Water-Supply Operations Simulation--Continued

*AGDMND (12)
CANLEN (100)
*CNVLAT
*CVLONG
*DRINK
*EVAP (12)
*PET (12)
*PREFAC
*PRSPFC
*RAIN (50)
RMILES (100)
*SEEPFC
*SESNAL (12)
*SNLAT1
*SNLAT2
*SNUOS1
*SNUOS2
*SNUOS3
*STCOEF
*TRANS
UNIT (5, 120)

PHYSIO

- monthly agricultural irrigation demand values

- length of canals

- conversion factor from degrees latitude to miles

- conversion factor from degrees longitude to miles

- average per person water demand

- monthly lake evaporation values

- monthly potential evapotranspiration values

- prestress factor to initialize return flows

- maximum rate of effective precipitation

- monthly rain at the rain gages

- river miles from downstream end of basin

- canal leakage rate

- monthly instream demand values

- latitude designation to distinguish SNUOS1 and SNUOS2

- latitude designation to distinguish SNUOS2 and SNUOS3

- senuosity factor to determine river length

- senuosity factor to determine river length

- senuosity factor to determine river length

- basinwide aquifer storage coefficient

- basinwide aquifer transmissivity

- unit responses for selected bands of SDF

\section{RESPON}

FRSPNZ $(100,480,2)$ - future ground-water flows to the river

\section{RESUSE}

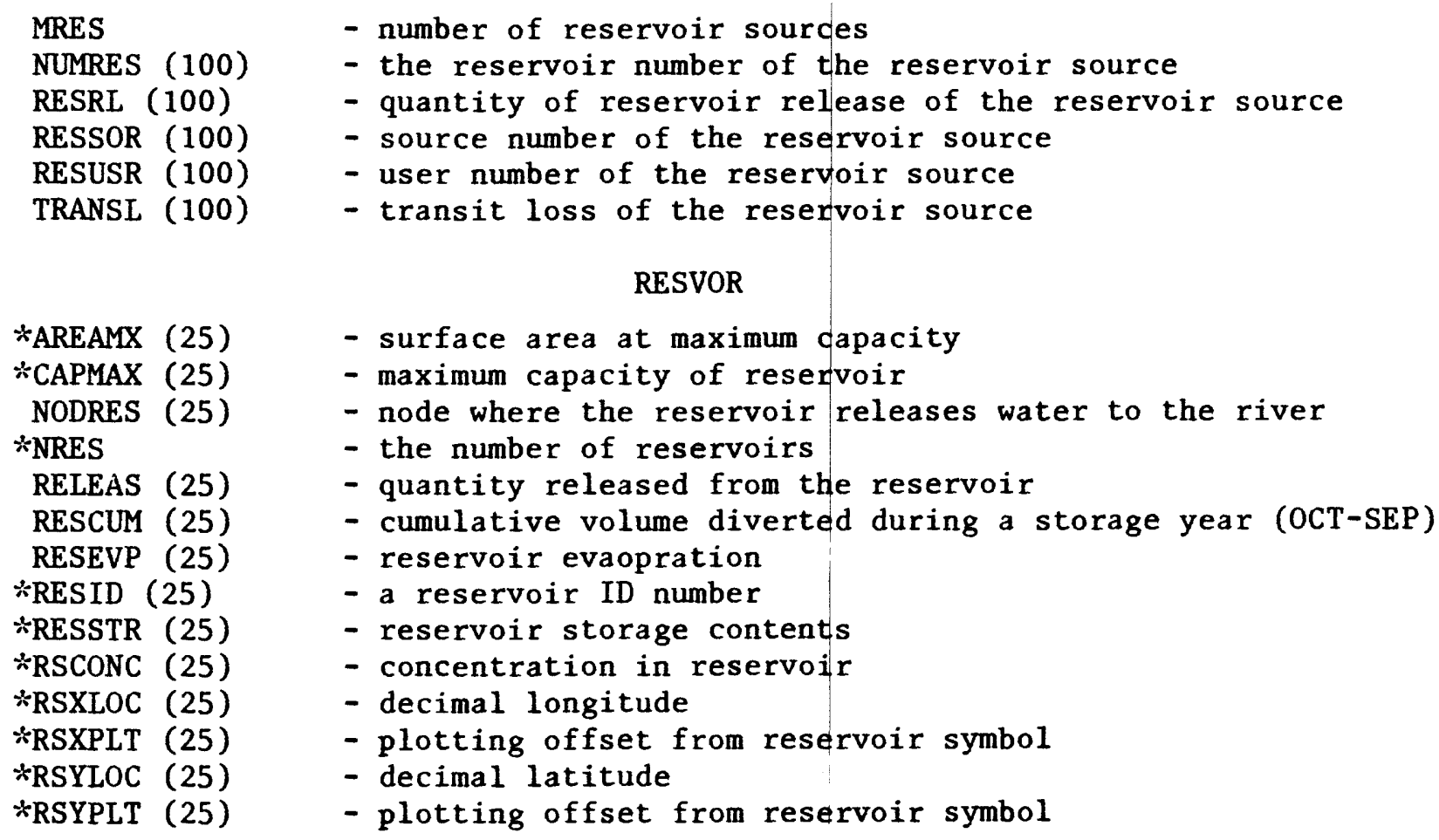

\section{RESVOR}

- surface area at maximum capacity

- maximum capacity of reservoir

- node where the reservoir releases water to the river

- the number of reservoirs

- quantity released from the reservoir

- cumulative volume diverted during a storage year (OCT-SEP)

- reservoir evaopration

- a reservoir ID number

- reservoir storage contents

- concentration in reservoir

- decimal longitude

- plotting offset from reservoir symbol

- decimal latitude

- plotting offset from reservoir symbol 
Attachment 6--Definition of Commoned Variables in Subroutines for Water-Supply Operations Simulation--Continued

RES 1

*RESNAM (25)

- an 8 character reservoir name

RIGHTS

*NRIGHT

*OWNER (200)

- the number of water rights

- user ID number (USERID)

\section{SKMTKU}

ACN $(100)$
ADD $(100)$
AGW $(100)$
AIM $(100)$
ANEED $(100)$
APR $(100)$
ARR $(100)$
*ISKMGW
*ISKMU
*UMAX

ニUMAX
- average consumptive use

- average direct diversions

- average ground-water pumpage

- average imports

- average water need

- average effective precipitation

- average reservoir releases

- code for frequency of plotting ground-water schematic

- code for frequency of plotting water-use schematic

- maximum water use associated with symbol size on the schematic

\section{STATR}

*ISSR (10)

RMEANC $(10,12)$
RMEAND $(10,12)$
RMEANE $(10,12)$
RMEANI $(10,12)$
RMEANL $(10,12)$
RMEANR $(10,12)$
RMEANS $(10,12)$

$\star$ ISSU (10)

UMENC $(10,12)$

UMENCL $(10,12)$

UMENDD $(10,12)$

UMENDP $(10,12)$

UMENGW $(10,12)$

UMENND $(10,12)$

UMENP $(10,12)$

UMENRR $(10,12)$

UMENTA $(10,12)$

UMENTW $(10,12)$

UMENWC $(10,12)$
- reservoir ID of those reservoirs for which statistical summaries will be printed

- monthly mean reservoir concentration

- monthly mean reservoir diversion inflow

- monthly mean reservoir evaporation

- monthly mean importation inflow

- monthly mean leakage

- monthly mean outflow release

- monthly mean storage contents

STATU

- water user ID of those water users for which statistical summaries will be printed

- monthly mean concentration of total applications

- monthly mean canal leakage

- monthly mean direct diversion

- monthly mean deep percolation

- monthly mean pumpage

- monthly mean water need

- monthly mean effective precipitation

- monthly mean reservoir release application

- monthly mean total application

- monthly mean tail water

- monthly mean consumptive use 
Attachment 6--Definition of Commoned Variables in Subroutines for Water-Supply Operations Simulation--Continued

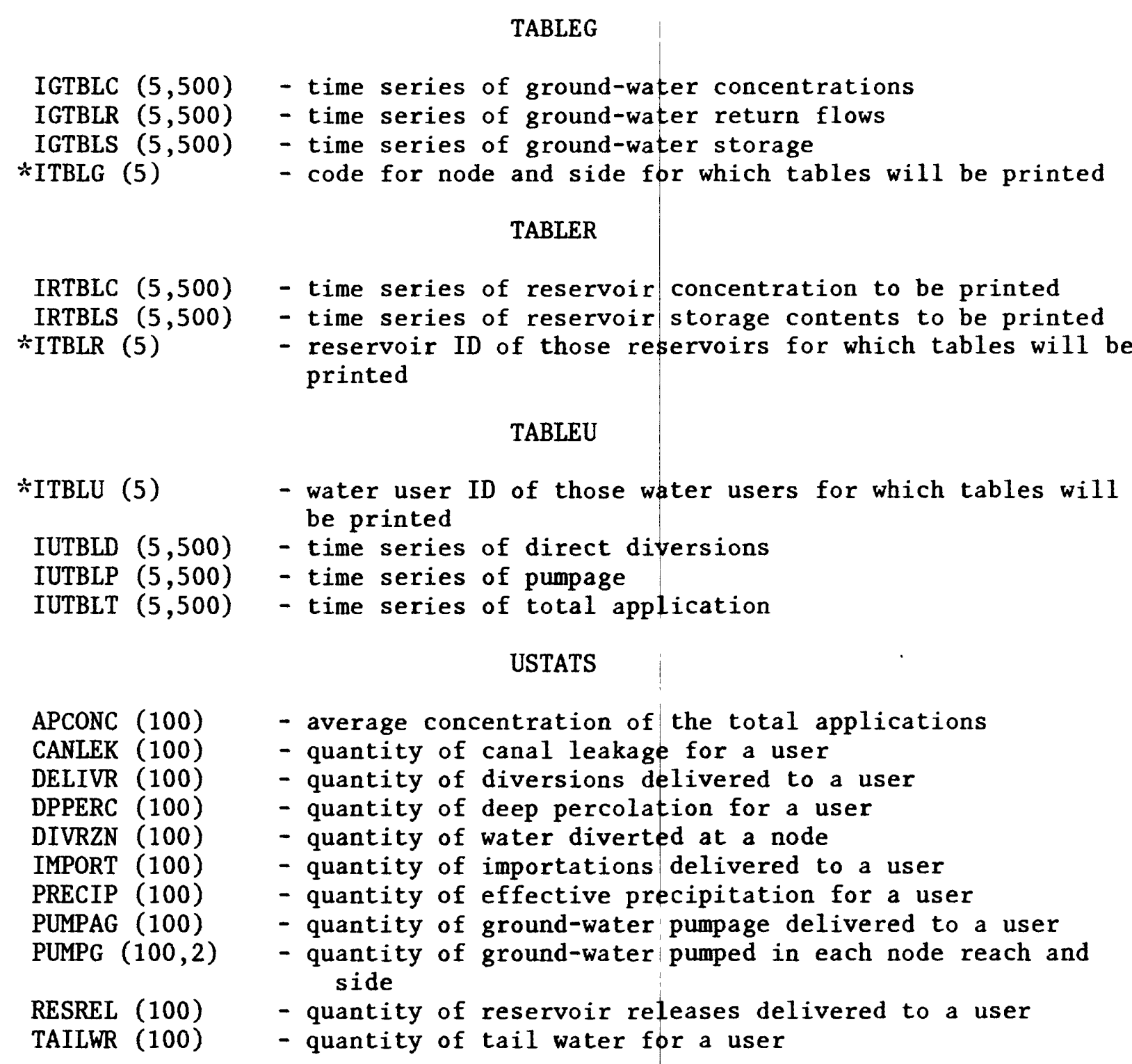


Attachment 7--FORTRAN SOURCE Code of Subroutines for

Streamflow and Water-Quality Simulation

CBSNMD2

C

C

C

C

C

C

C

COMMON NMONTH, NINDV , IN , IOUT , IPRINT , I GRAPH, IBATCH, IQW , IMONTH, 1 JMONTH, IYEAR COMMON /NAMES/ TITL(5)

COMMON /REACHS/ NNODE, NODEID (100), NXTNOD (100), XLOC (100), YLOC (100), 1 $\operatorname{PLTX}(100), \operatorname{PLTY}(100), \operatorname{NTRCPT}(100,12), \operatorname{SLOPE}(100,12)$, QWA $(100,12), Q W B(100,12), \operatorname{INDEPV}(100,12), \mathrm{Q}(100)$, QW(100), CONCFC (100), NODEUS (100), QSUBA (100),

4

REAL NTRCPT QWSUBA (100), DSA(100), DSB(100)

COMMON /SCHMTK/ ISKM,PORQ(100), PORQW(100), AQ(100), AQW(100), 1 QHYMAX (5), PORXN (100), AXN (100)

DIMENSION ITITLE (20)

C CHARACTER $* 80$ IDUM

REAL ITITLE

C EQUIVALENCE (IDUM, ITITLE)

C

$$
\begin{aligned}
& \text { IN }=1 \\
& \text { IOUT }=1
\end{aligned}
$$

WRITE (IOUT, 1000)

1000 FORMAT (54H IS THIS AN INTERACTIVE SESSION (0 FOR NO, 1 FOR YES)?) CALL QUESZN (IBATCH)

C
WRITE (IOUT, 1010)

\section{FORMAT (44H}

$156 \mathrm{H}$

$240 \mathrm{H}$

IF (IBATCH .EQ.

C

WRITE (IOUT, 1020)

1020 FORMAT (53H FOR ALL QUESTIONS, 1 = YES AND ANY OTHER NUMBER = NO $1, /, 37 \mathrm{H}$ DO YOU WANT THE GENERAL EXPLANATION? )

CALL QUESZN (IQ)

IF (IQ - 1) $20,10,20$

10 WRITE (IOUT, 1030)

1030 FORMAT $(/ /, 65 \mathrm{H}$

THIS MODEL IS THE SECOND IN A PLANNED SERIES 0 $1 F$ RIVER BASIN,/,65H SIMULATION MODELS. VERSION \#2 IS A RATHER SIM 2PLE ROUTING MODEL., $/, 66 \mathrm{H}$ FLOW FROM THE INTERMEDIATE DRAINAGE AREA 3OF EACH NODE IS COMPUTED, /,61H WITH REGRESSION PARAMETERS AND A SE 4T OF TEMPORAL INDEPENDENT ,/,66H VARIABLES. REGRESSION PARAMETERS 5 ARE ALSO NEEDED TO DESCRIBE THE ,/,63H WATER QUALITY-DISCHARGE RE 6LATIONSHIPS. INTERMEDIATE DISCHARGE,/,65H FROM EACH NODE IS THEN 7ACCUMULATED AND ROUTED DOWNSTREAM. WATER, /,62H QUALITY CONCENTRAT 8IONS ARE MIXED DURING EACH FLOW ROUTING AND,/,20H LOADS ARE COMPUT 9ED. , ///) 
Attachment 7--FORTRAN SOURCE Code of Subroutines for

Streamflow and Water-Quality Simulation--Continued

C

20 WRITE (IOUT, 1040)

1040 FORMAT (30H DO YOU WANT THE INSTRUCTIONS? )

CALL QUESZN (IQ)

IF (IQ - 1) $40,30,40$

C

30 CALL INSTRK

40 IF (IBATCH .EQ. 1) CALL READFI

IF (IBATCH .NE. 1) CALL READFB

C

C INITIALIZATION

$\mathrm{C}$

IYEAR $=0$

DO 50 INODE $=1$, NNODE

$\operatorname{PORXN}($ INODE $)=0$.

PORQW (INODE) $=0$.

C

50 PORQ (INODE) $=0$.

IF (ISKM .NE. 1) GO TO 60

WRITE (IDUM, 1050) TITL

1050 FORMAT (5A4,22H - MONTHLY SIMULATION\$ )

CALL MNTHLY (ITITLE)

$\mathrm{C}$

DO MONTHLY SIMULATION OVER ALL MONTHS

$\mathrm{C}$

60 DO 100 IMONTH $=1$, NMONTH

JMONTH $=$ IMONTH $-(($ IMONTH -1$) / 12) \div 12$

IF (JMONTH - 1) $70,70,90$

70 DO 80 INODE $=1$, NNODE

$\operatorname{AXN}($ INODE) $=0$.

$\mathrm{AQW}($ INODE $)=0$.

$80 \mathrm{AQ}(\mathrm{INODE})=0$.

IYEAR = IYEAR + 1

IF (IBATCH .EQ. 1 .AND. ISKM .NE. 1) WRITE (IOUT, 1060) IYEAR

1060 FORMAT (26H CURRENTLY SIMULATING YEAR ,I3)

C

90 CALL NFLOW

CALL SUMRYM

100 CONTINUE

C

C SUMMARIZE AND DO ANY DESIRED COMPARISONS

C

CALL SUMRYF

110 IF (IBATCH .EQ. 0) GO TO 120

WRITE (IOUT, 1070)

1070 FORMAT (66H DO YOU WANT TO COMPARE RESULTS FROM THIS RUN WITH A PR 1EVIOUS RUN? )

120 CALL QUESZN(IQ)

IF (IQ - 1) $140,130,140$

130 CALL CMPAR

GO TO 110

140 CONTINUE

IF (IGRAPH .NE. O) CALL DONEPL

C

STOP

END 
Attachment 7--FORTRAN SOURCE Code of Subroutines for

Streamflow and Water-Quality Simulation--Continued

111 CCMPAR

$112 \mathrm{C}$

$113 \mathrm{C}$

$114 \quad C$

$115 \mathrm{C}$

$116 \quad \mathrm{C}$

$117 \quad \mathrm{C}$

SUBROUTINE COMPARISON OF RIVER BASIN SIMULATION MODEL (\#2)

WRITTEN BY ALAN BURNS, U.S. GEOLOGICAL SURVEY

WATER RESOURCES DIVISION, LAKEWOOD, CO

LATEST UPDATE $1 / 22 / 87$

118

119

120

121

122

123

124

125

126

127

128

129

130

131

132

133

134

135

136

137

138

139

140

141

142

143

144

145

146

147

148

149

150

151

152

153

154

155

156

157

158

159

160

161

SUBROUTINE CMPAR

COMMON NMONTH, NINDV , IN, IOUT, IPRINT , IGRAPH, IBATCH, IQW, IMONTH, 1 JMONTH, I YEAR

COMMON /NAMES/ TITL(5)

COMMON /REACHS/ NNODE, NODEID (100), NXTNOD $(100), \operatorname{XLOC}(100), \operatorname{YLOC}(100)$,

1

2 PLTX (100), PLTY (100), NTRCPT $(100,12), \operatorname{SLOPE}(100,12)$,

3

4

REAL NTRCPT

$\operatorname{QWA}(100,12), \operatorname{QWB}(100,12), \operatorname{INDEPV}(100,12), Q(100)$, QW(100), CONCFC (100), NODEUS (100), QSUBA(100), QWSUBA(100),DSA(100), DSB(100)

COMMON /REACHI/ NODNAM(100)

DOUBLE PRECISION NODNAM

C

DIMENSION TITL2(5), $\operatorname{YTEMP}(3), \mathrm{X} 1(600), \mathrm{Y1}(600), \mathrm{X} 2(600), \mathrm{Y} 2(600)$,

$1 \mathrm{X} 3(600), Y 3(600)$, ITITLE (20), TITLED (20), TITLEP (20)

DIMENSION ERAV (12), ERNM (12), ERMX (12), ERMN (12), MONMX (12), MOMMN (12)

CHARACTER $* 80$ IDUM

CHARACTER*6 LXNAME

C

DOUBLE PRECISION SD,ERRSD, XXN

REAL ITITLE

EQUIVALENCE (IDUM, ITITLE)

C

REWIND 63

REWIND 64

C

C

SKIP DOWN TO THE MONTHLY OUTPUT

C

READ $(63,1000)$ ITEST, ITEST, ITEST, ITEST

READ $(64,1000)$ ITEST, ITEST, ITEST

1000 FORMAT (1A1)

READ $(64,1010)$ TITL2

1010 FORMAT (20X,5A4)

WRITE (IOUT, 1020) TITL2

1020 FORHAT $(1 / 147 \mathrm{H}$ THE PREVIOUS RUN YOU LOADED FOR COMPARISON IS: ,5A4)

READ $(63,1000)$ ITEST, ITEST, ITEST

READ $(64,1000)$ ITEST, ITEST

READ $(64,1030)$ TITLED, NNODEP

1030 FORMAT $(26 \mathrm{X}, 20 \mathrm{~A} 4,11 \mathrm{X}, \mathrm{I} 3)$

WRITE (IOUT, 1040) TITLED

1040 FORMAT (32H ITS BASIN DESCRIPTION FILE WAS: ,/,20A4)

READ $(63,1000)$ ITEST, ITEST, ITEST, ITEST

READ $(64,1000)$ ITEST, ITEST

READ $(64,1050)$ TITLEP, NINDVP, MONTHP

1050 FORMAT $(29 \mathrm{X}, 20 \mathrm{~A} 4, /, 18 \mathrm{X}, \mathrm{I3}, 13 \mathrm{X}, \mathrm{I3})$

WRITE (IOUT, 1060) TITLEP

1060 FORMAT (35H ITS INDEPENDENT VARIABLE FILE WAS: ,/,20A4) 
Attachment 7--FORTRAN SOURCE Code of Subroutines for

Streamflow and Water-Quality Simulation--Continued

165

166

167

168

169

170

171

172

173

174

175

176

177

178

179

180

181

182

183

184

185

186

187

188

189

190

191

192

193

194

195

196

197

198

199

200

201

202

203

204

205

206

207

208

209

210

211

212

213

214

215

216

217

218

219

220

READ $(63,1000)$ ITEST, ITEST, ITEST, ITEST, ITEST

READ $(64,1000)$ ITEST, ITEST, ITEST, ITEST, ITEST

DO $10 I=1$, NNODE

$10 \operatorname{READ}(63,1000)$ ITEST

DO $20 \mathrm{I}=1$, NNODEP

$20 \operatorname{READ}(64,1000)$ ITEST

DO $30 I=1$, NNODE

DO 30 LINES $=1,17$

30 READ $(63,1000)$ ITEST

DO $40 \mathrm{I}=1$, NNODEP

DO 40 LINES $=1,17$

$40 \operatorname{READ}(64,1000)$ ITEST

IF (IBATCH .EQ. 0) GO TO 50

WRITE (IOUT, 1070)

1070 FORMAT (27H ENTER THE NODE ID (5 COLS) )

50 READ (IN, 1080) INODE

1080 FORMAT (I5 , A8, 2X, 2F 10.0, I5 , 3F 10.0)

DO 60 IINODE $=1$, NNODE

IF (NODEID(IINODE) .NE. INODE) GO TO 60

GO TO 70

60 CONTINUE

70 IF (IBATCH .EQ. 0) GO TO 80

WRITE (IOUT, 1090)

1090 FORMAT (5OH ENTER 1 FOR FLOW, 2 FOR CONCENTRATION, 3 FOR LOAD, $18 \mathrm{H}(1 \mathrm{COL}))$

80 READ (IN, 1100) ITYPE

1100 FORMAT (I1)

GO TO $(90,100,110)$, ITYPE

90 WRITE (IOUT, 1110) NODEID(I INODE), NODNAM(IINODE)

1110 FORMAT (47H THE FLOWS, IN CUBIC FEET PER SECOND, FOR NODE , I5,2H ( $1, \mathrm{~A} 8,14 \mathrm{H})$ ARE COMPARED )

GO TO 120

100 WRITE (IOUT, 1120) NODE ID(IINODE), NODNAM(IINODE)

1120 FORMAT (54H THE CONCENTRATIONS, IN MILLIGRAMS PER LITER, FOR NODE 1, I5, 2H $($, A8, 14H) ARE COMPARED )

GO TO 120

110 WRITE (IOUT, 1130) NODEID(IINODE), NODNAM(IINODE)

1130 FORMAT (3OH THE LOADS, IN TONS, FOR NODE , I5, 2H (,A8,14H) ARE COMP IARED )

C

120 YMAX $=0$.

YMTN $=0$.

DIFMAX $=0$.

DIFMIN $=1.0 \mathrm{E} 10$

XMONTH $=0$.

MONTHX $=0$

MONTH1 $=1$

MONTH2 $=1$

ERRMN $=0$.

ERRSD $=0$.

DO $125 \mathrm{~J}=1,12$

$\operatorname{ERAV}(\mathrm{J})=0$.

$\operatorname{ERNM}(\mathrm{J})=0$.

$\operatorname{ERMX}(\mathrm{J})=-1.0 \mathrm{E} 10$

$125 \operatorname{ERMN}(\mathrm{J})=1.0 \mathrm{E} 10$

MONTH $=$ MMONTH 
Attachment 7--FORTRAN SOURCE Code of Subroutines for Streamflow and Water-Quality Simulation--Continued

$$
\text { C }
$$

C

C

C

$$
\text { READ AND SELECT DESIRED PARAMETER }
$$

DO 160 IMONTH $=1$, MONTH

JMONTH $=$ IMONTH $-(($ IMONTH -1$) / 12) * 12$

READ $(63,1000)$ ITEST, ITEST, ITEST, ITEST, I TEST, ITEST

READ $(64,1000)$ ITEST, ITEST, ITEST, ITEST, ITEST, ITEST

DO $130 \mathrm{I}=1$, NNODE

READ $(63,1140)$ NODE, YTEMP

1140 FORMAT $(6 \mathrm{X}, \mathrm{I7}, 10 \mathrm{X}, \mathrm{F} 11.2, \mathrm{~F} 13.0, \mathrm{~F} 11.2)$

IF (NODE .NE. INODE) GO TO 130

Y1 (MONTH1) $=$ YTEMP (ITYPE)

IF (YTEMP (ITYPE) .EQ. -99.) GO TO 130

IF (YTEMP (ITYPE) .GT. YMAX) YMAX = YTEMP (ITYPE)

130 CONTINUE

$c$

DO $140 I=1$, NNODEP

READ $(64,1140)$ NODE , YTEMP

IF (NODE .NE. INODE) GO TO 140

Y2 $($ MONTH2) $=$ YTEMP (ITYPE)

IF (YTEMP(ITYPE) .EQ. -99.) GO TO 140

IF (YTEMP (ITYPE) .GT. YMAX) YMAX = YTEMP (ITYPE)

C

$X 1($ MONTH1) $=$ IMONTH

$\mathrm{X} 1($ MONTH 1$)=\mathrm{X} 1$ (MONTH1) -.25

$\mathrm{X} 2($ MONTH2) $=\mathrm{X} 1($ MONTH 1$)+.5$

IF (Y1(MONTH1) .EQ. -99.) GO TO 150

MONTH1 $=$ MONTH1 +1

IF (Y2(MONTH2) .EQ. -99.) GO TO 160

MONTH2 $=$ MONTH2 +1

$\mathrm{ERR}=\mathrm{Y} 1($ MONTH1 - 1) $-\mathrm{Y} 2($ MONTH2 - 1)

IF (ERR .GT. DIFMAX) DIFMAX = ERR

IF (ERR .IT. DIFMIN) DIFMIN $=$ ERR

XMONTH $=$ XMONTH +1 .

MONTHX $=$ MONTHX +1

$\mathrm{X} 3$ (MONTHX) $=$ IMONTH

Y3 (MONTHX) = ERR

ERRMN = ERRMN + ERR

ERRSD" $=$ ERRSD + ERR*ERR

$\operatorname{ERNM}($ JMONTH) $=\operatorname{ERNM}($ MMONTH $)+1$.

$\operatorname{ERAV}($ MMONTH $)=\operatorname{ERAV}($ MMNTH) $+\operatorname{ERR}$

IF (ERR . GT . ERMX (JMONTH)) MONMX (JMONTH) $=$ IMONTH

IF (ERR .GT. ERMX (JMONTH)) ERMX (JMONTH) = ERR

IF (ERR . IT . ERMN (JMONTH)) MONMN (JMONTH) = IMONTH

IF (ERR .LT . ERMN (JMONTH)) ERMN(JMONTH) $=$ ERR

GO TO 160

150 IF (Y2(MONTH2) .EQ. -99.) GO TO 160

MONTH2 $=$ MONTH $2+1$

C

160 CONTINUE

IF (XMONTH .LE. 1.) RETURN

MONTH $1=$ MONTH $1-1$

MONTH2 $=$ MONTH2 -1 
Attachment 7--FORTRAN SOURCE Code of Subroutines for

Streamflow and Water-Quality Simulation--Continued

SD $=$ ERRMN*ERRMN $/$ XMONTH

$X X N=X M O N T H-1$.

ERRSD $=$ DSQRT $((E R R S D-S D) / X X N)$

ERRMN = ERRMN $/$ XMONTH

WRITE (IOUT, 1150) ERRMN, ERRSD

1150 FORMAT (43H THE MEAN RESIDUAL BETWEEN THE TWO RUNS IS ,F10.3,

$1 /, 30 \mathrm{H}$ THE STANDARD DEVIATION IS ,F10.3)

WRITE (IOUT , 1155) (J,ERAV(J)/ERNM(J), ERMX (J), MONMX (J), ERMN (J),

$1 \operatorname{MONMN}(\mathrm{J}), \mathrm{J}=1,12)$

1155 FORMAT (15X,46HMONTH AVERAGE MAXIMUM MONTH MINIMUM MONTH ,/,

$1(15 X, I 3, F 11.2, F 9.1, I 6, F 10.1, I 6))$

IF (IGRAPH .EQ. O) RETURN

C

C

C

PLOT THE TWO SETS OF DATA AS HYDROGRAPHS

$X M I N=0$.

$\mathrm{XMAX}=\mathrm{MONTH}+1$

IF (MONTH .GT. 37) XMAX $=$ XMAX/12.

$\mathrm{XINC}=\mathrm{FINC}(\mathrm{XMAX}, \mathrm{XMIN})$

IF (MONTH .GT. 37) XMAX $=$ XMAX*12.

YINC $=$ FINC (YMAX, YMIN $)$

CALL RESET ('ALL')

CALL PAGE $(11,8.5)$

CALL NOBRDR

CALL AREA2D $(10,6.0)$

CALL INTAXS

WRITE (IDUM, 1160) NODEID(IINODE), NODNAM(IINODE)

1160 FORMAT $(\mathrm{I5}, 2 \mathrm{H}(, \mathrm{A} 8,2 \mathrm{H}) \$)$

CALL HEIGHT (.2)

CALL HEADIN (ITITLE, $100,1 ., 1$ )

LXNAME $=$ 'MONTHS'

IF (MONTH .GT. 37) LXNAME = 'YEARS '

CALL XNAME (LXNAME, 6)

IF (ITYPE .EQ. 1) CALL YNAMB (36HSTREAMFLOW, IN CUBIC FEET PER SECO 1ND , 36)

IF (ITYPE .EQ. 2) CALL YNAME(38HCONCENTRATION, IN MILLIGRAMS PER L IITER ,38)

IF (ITYPE .EQ. 3) CALL YNAME (23HLOAD, IN TONS PER MONTH ,23)

CALL XTICKS $(0)$

CALL YTICKS (0)

CALL XNONUM

CALL GRAF (XMIN , XINC , XMAX, YMIN, YINC, MMAX)

CALL BARS $(-.25)$

CALL SETCLR (3HRED)

CALL MESSAG(TITL, 20,4.0,5.7)

CALL CURVE (X1, Y1, MONTH 1,0)

CALL SETCLR (5HGREEN)

CALL MESSAG(TITL2, 20,4.0,5.4)

CALL CURVE (X2,Y2, MONTH2,0)

IF (MONTH .GT. 37) XMAX $=$ XMAX/12.

CALL SETCLR(5HWHITE)

CALL RESET (' XNONUM')

CALL XGRAXS (XMIN, XINC, XMAX, 10., IH , 1,0.,0.)

ITICKS $=$ XINC +.1

CALL XTICKS (ITICKS)

CALL RESET ('YTICKS') 
Attachment 7--FORTRAN SOURCE Code of Subroutines for

Streamflow and Water-Quality Simulation--Continued

CALL XNONUM

CALL YNONUM

CALL XGRAXS (XMIN,XINC, XMAX, 10.,1H ,-1,0.,0.)

CALL YGRAXS (YMIN, YINC, YMAX, $6.0,1 \mathrm{H},-1,0 ., 0$. )

CALL XGRAXS (XMIN, XINC, XMAX, 10.,1H , 1,0.,6.0)

CALL YGRAXS (YMIN, YINC, YMAX $, 6.0,1 \mathrm{H}, 1,10 ., 0$. )

CALL ENDPL(0)

C

C

PLOT THE TWO SETS OF DATA AS FREQUENCY CURVES

CALL SORT (Y1, MONTH1)

CALL PROB (X1,MONTH 1$)$

CALL SORT (Y2,MONTH2)

CALL PROB (X2,MONTH2)

CALL RESET ('ALL')

CALL PAGE $(11,8.5)$

CALL NOBRDR

CALL AREA2D $(10,6.0)$

CALL INTAXS

CALL HEIGHT (.2)

CALL HEADIN (ITITLE, $100,1,1$ )

CALL XNAME (26HPERCENT OF MONTHS EXCEEDED ,26)

IF (ITYPE .EQ. 1) CALL YNAME (36HSTREAMFLOW, IN CUBIC FEET PER SECO 1ND ,36)

IF (ITYPE .EQ. 2) CALL YNAME(38HCONCENTRATION,. IN MILLIGRAMS PER L 1ITER ,38)

IF (ITYPE .EQ. 3) CALL YNAME (23HLOAD, IN TONS PER MONTH ,23)

$\mathrm{XMIN}=\mathrm{X} 1(1)$

IF $(\mathrm{X} 2(1) \cdot \mathrm{GT} . \mathrm{XMIN}) \mathrm{XMIN}=\mathrm{X} 2(1)$

$\mathrm{XMAX}=\mathrm{X} 1(\mathrm{MONTH} 1)$

IF $(X 2($ MONTH2) . LT. XMAX) XMAX $=$ X2 (MONTH2)

$\mathrm{XINC}=\mathrm{XMAX}-\mathrm{XMIN}$

CALL XNONUM

CALL XTICKS (0)

CALL YTICKS $(0)$

CALL GRAF (XMIN, XINC, XMAX, YMIN, YINC, YMAX)

CALL SETCLR(3HRED)

CALL MESSAG(TITL , 20,2.5,5.7)

CALL CURVE (X1, Y1, MONTH 1,0$)$

CALL HWSHD

CALL SHDPAT (1)

CALL SHDCRV (X1, Y1, MONTH1 , X1 , Y1 , 5HBELOW)

CALL SETCLR (5HGREEN)

CALL MESSAG(TITL2, 20, 2.5,5.4)

CALL CURVE (X2, Y2, MONTH2,0)

CALL RESET ('HWSHD')

CALL SHDPAT (3)

CALL SHDCRV (X2, Y2, MONTH2, X2, Y2, 5HBELOW)

CALL SETCLR (5HWHITE)

CALL RESET('YTICKS')

CALL YNONUM

CALL RLVEC (XMIN, YMIN, XMAX, YMIN , 0)

CALL YGRAXS (YMIN, YINC, YMAX, 6.0,1H , - 1, 0. ,0.)

CALL RLVEC (XMIN, YMAX, XMAX, YMAX, 0 )

CALL YGRAXS (YMIN, YINC, YMAX , 6.0,1H , 1, 10 . , 0.)

MONTH $=$ MONTH 1 
Attachment 7--FORTRAN SOURCE Code of Subroutines for

C

C

C Streamflow and Water-Quality Simulation--Continued IF (MONTH2 .GT. MONTH) MONTH $=$ MONTH2

CALL RKRLAB (MONTH, YMAX, YMIN)

CALL ENDPL(0)

\section{PLOT A HYDROGRAPH OF THE RESIDUALS}

MONTH $=$ NMONTH

IF (MONTHP .LT. MONTH) MONTH $=$ MONTHP

XMIN $=0$.

XMAX $=$ MONTH +1

IF (MONTH .GT. 37) XMAX $=$ XMAX/12.

$\mathrm{XINC}=\mathrm{FINC}(\mathrm{XMAX}, \mathrm{XMIN})$

IF (MONTH .GT. 37) XMAX = XMAX $\div 12$.

DIFINC $=$ FINC (DIFMAX, DIFMIN $)$

CALL RESET ('ALL')

CALL PAGE $(11,8.5)$

CALL NOBRDR

CALL AREA2D $(10,6.0)$

CALL HEIGHT $(.2)$

CALL INTAXS

CALL HEADIN(ITITLE, $100,1 ., 1$ )

CALL XNAME $(1 \mathrm{H}, 1)$

WRITE (IDUM, 1160) NODEID(I INODE), NODNAM (IINODE)

IF (ITYPE .EQ. 1) CALL YNAME (36HSTREAMFLOW, IN CUBIC FEET PER SECO 1ND , 36)

IF (ITYPE .EQ. 2) CALL YNAME (38HCONCENTRATION, IN MILLIGRAMS PER L IITER ,38)

IF (ITYPE .EQ. 3) CALL YNAME (23HLOAD, IN TONS PER MONTH ,23)

CALL CROSS

CALL XNONUM

CALL XTICKS $(0)$

CALL YTICKS $(0)$

CALL GRAF (XMIN, XINC, XMAX,DIFMIN, DIFINC, DIFMAX)

CALL MESSAG(TITL $, 20,2.0,6.1$ )

CALL MESSAG(TITL2, 20,6.0,6.1)

CALL SETCIR (4HCYAN)

CALL BARPAT (16)

CALL BARWID $(-1$.

CALL VBARS (X3, Y3, 'BASE', MONTHX)

IF (MONTH .GT. 37) XMAX $=X M A X / 12$.

CALL SETCLR(5HWHITE)

ITICKS $=$ XINC +.1

CALL XTICKS (ITICKS)

CALL RESET ('YTICKS')

CALL YNONUM

CALL XGRAXS (XMIN, XINC, XMAX, 10.,1H , -1,0.,0.)

CALL YGRAXS (DIFMIN,DIFINC,DIFMAX, $6.0,1 \mathrm{H},-1,0 ., 0$. )

CALL XGRAXS (XMIN, XINC, XMAX, 10.,1H , 1,0.,6.0)

CALL YGRAXS (DIFMIN,DIFINC,DIFMAX $, 6.0,1 \mathrm{H}, 1,10 ., 0$. )

CALL RESET ('XNONUM')

CALL XTICKS $(0)$

IF (MONTH.LT . 38) CALL XGRAXS (XMIN, XINC, XMAX, 10 , 6HMONTHS , 6, 0. , 0. )

IF (MONTH.GT. 37) CALL XGRAXS (XMIN, XINC, XMAX, 10. ,5HYEARS , 5, 0. , 0.)

C CALL ENDPL(0) 
Attachment 7--FORTRAN SOURCE Code of Subroutines for

Streamflow and Water-Quality Simulation--Continued

446 CHYDRGR

447 C

$448 \quad \mathrm{C}$

$449 \mathrm{C}$

$450 \quad \mathrm{C}$

$451 \quad \mathrm{C}$

$452 \quad \mathrm{C}$

453

454

455

456

457

458

459

460

461

462

463

464

465

466

467

468

469

470

471

472

473

474

475

476

477

478

479

480

481

482

483

484

485

486

487

488

489

490

491

492

493

494

495

496

C

SUBROUTINE HYDROGRAPH OF RIVER BASIN SIMULATION MODEL

WRITTEN BY ALAN BURNS, U.S. GEOLOGICAL SURVEY WATER RESOURCES DIVISION, LAKEWOOD, CO LATEST UPDATE $1 / 22 / 87$

SUBROUTINE HYDRGR(Q, ITITLE, ITYPE)

COMMON NMONTH, NINDV, IN , IOUT, IPRINT, I GRAPH, IBATCH, IQW, IMONTH,

1

$$
\text { JMONTH, IYEAR }
$$

DIMENSION $Q(1), \operatorname{ITITLE}(1)$

DIMENSION XMON(600)

CHARACTER $* 6$ LXNAME

C

DO $10 \mathrm{I}=1, \mathrm{NMONTH}$

$10 \mathrm{XMON}(\mathrm{I})=\mathrm{I}$

XMIN $=0$

XMAX $=$ NMONTH

IF (NMONTH .GT. 37) XMAX $=$ XMAX/12.

$\mathrm{XINC}=\operatorname{FINC}(\mathrm{XMAX}, \mathrm{XMIN})$

IF (NMONTH .GT. 37) XMAX $=X M A X * 12$.

YMIN $=0$.

C

YMAX $=Q(1)$

DO 20 IMONTH $=2$, NMONTH

IF ( $Q$ (IMONTH) .GT. YMAX) YMAX $=Q$ (IMONTH)

20 CONTINUE

YINC $=\operatorname{FINC}($ YMAX, YMIN $)$

C

CALL RESET ('ALL')

CALL RESET ('ALL')

CALL PAGE $(11 ., 8.5)$

CALL NOBRDR

CALL AREA2D $(10,6.0)$

CALL INTAXS

LXNAME $=$ 'MONTHS'

IF (NMONTH .GT. 37) LXNAME = 'YEARS '

CALL HEIGHT (.2)

CALL HEADIN (ITITLE , 100,1.,1)

CALL XNAME (LXNAME, 6)

IF (ITYPE .EQ. 1) CALL YNAME (36HSTREAMFLOW, IN CUBIC FEET PER SECO 1ND ,36)

IF (ITYPE .EQ. 2) CALL YNAME (38HCONCENTRATION, IN MILLIGRAMS PER L IITER ,38)

IF (ITYPE .EQ. 3) CALL YNAME (23HLOAD, IN TONS PER MONTH ,23)

IF (ITYPE .EQ. 4) CALI YNAME (34HDIVERSIONS, IN ACRE-FEET PER MONTH $1,34)$

IF (ITYPE .EQ. 5) CALL YNAME (31HPUMPAGE, IN ACRE-FEET PER MONTH, $131)$

IF (ITYPE .EQ. 6) CALL YNAME (42HTOTAL APPLICATIONS, IN ACRE-FEET P IER MONTH , 42)

IF (ITYPE .EQ. 7) CALL YNAME(22HCONTENTS, IN ACRE-FEET ,22) 
Attachment 7--FORTRAN SOURCE Code of Subroutines for

Streamflow and Water-Quality Simulation--Continued

499

500

501

502

503

504

505

506

507

508

509

510

511

512

513

514

515

516

517

518

519

520

521

522

523

524

525

526

IF (ITYPE .EQ . 8) CALL YNAME (37HRETURN FLOW, IN CUBIC FEET PER SEC 10ND ,37)

CALL XTICKS $(0)$

CALL YTICKS (0)

CALL XNONUM

CALL GRAF (XMIN, XINC, XMAX, YMIN, YINC, YMAX)

CALL HWSHD

CALL BARPAT (16)

CALL SETCLR (3HRED)

CALL BARWID $(-1$.

CALL VBARS (XMON, Q, 'BASE', NMONTH)

IF (NMONTH .GT. 37) XMAX $=X M A X / 12$.

CALL SETCLR(5HWHITE)

CALL RESET ('XNONUM')

CALL XGRAXS (XMIN, XINC , XMAX, 10., 1H , 1,0.,0.)

CALL XNONUM

CALL YNONUM

CALL RESET('YTICKS')

ITICKS $=$ XINC +.1

CALL XTICKS (ITICKS)

CALL XGRAXS (XMIN, XINC, XMAX, 10., 1H , -1,0.,0.)

CALL YGRAXS (YMIN, YINC, YMAX, 6.0,1H ,-1,0.,0.)

CALL XGRAXS (XMIN,XINC, XMAX, 10.,1H ,1,0.,6.0)

CALL YGRAXS (YMIN, YINC, YMAX, 6.0, 1H , 1, 10.,0.)

C

CALL ENDPL(0)

RETURN

END 
Attachment 7--FORTRAN SOURCE Code of Subroutines for

Streamflow and Water-Quality Simulation--Continued

527 CINSTRK

$528 \mathrm{C}$

$529 \quad \mathrm{C}$

$530 \quad \mathrm{C}$

$531 \quad \mathrm{C}$

532 C

$533 \mathrm{C}$

534

535

536

537

538

539

540

541

542

543

544

545

546

547

548

549

550

551

552

553

554

555

556

557

558

559

560

561

562

563

564

565

566

567

568

569

570

571

572

573

574

575

576

577

C

SUBROUTINE INSTRUCTION OF RIVER BASIN SIMULATION MODEL (非)

WRITTEN BY ALAN BURNS, U.S. GEOLOGICAL SURVEY

WATER RESOURCES DIVISION, LAKEWOOD, CO

LATEST UPDATE $9 / 21 / 87$

SUBROUTINE INSTRK

COMMON NMONTH, NINDV , IN , IOUT, IPRINT , I GRAPH, IBATCH, IQW, IMONTH, 1 JMONTH, IYEAR

WRITE (IOUT, 1000)

1000 FORMAT (73H Three data sets must be prepared prior to using the mo ldel for streamflow ,/, 12H simulation: ,/,

$249 \mathrm{H}$

$356 \mathrm{H}$

A physical description of the modeled area; ,/,

$453 \mathrm{H}$

a digitized network of points along the river; and ,/,

$549 \mathrm{H}$ The format for the physical description file is: ,/,

6 37H Record Columns Name Description,/,

$780 \mathrm{H}+$

8

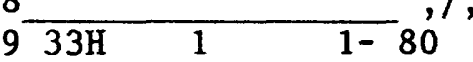

* $62 \mathrm{H} 2 \mathrm{l}$

$1-5$ NNODE the number of nodes (maximum of 100)

$1, /$,

$278 \mathrm{H} 3$ 1- 10 QMAX an estimate of the largest streamflo

$3 w$, in cubic feet, /,

$431 X, 39 H p e r$ second (used for plotting purposes) ,/,

$577 \mathrm{H} \quad 11-20$ QWL1 value of water quality, in milligram

$6 \mathrm{~s}$ per liter, to ,/,

$731 \mathrm{X}, 30 \mathrm{Hsubdivide}$ class 1 from class 2 )

WRITE (IOUT, 1010)

1010 FORMAT $(77 \mathrm{H} \quad 21-30$ QWL2 value of water quality, in mi

111 igrams per liter, to ,/,

$231 \mathrm{X}, 30 \mathrm{Hsubdivide}$ class 2 from class 3 ,/,

$377 \mathrm{H} \quad 31-40$ QWL3 value of water quality, in milligram

$4 \mathrm{~s}$ per liter, to,/,

$531 \mathrm{X}, 30 \mathrm{Hsubdivide}$ class 3 from class 4 ,/,

$665 \mathrm{H}$ note -- records 4 and 5 are entered as a set, one for each NN $700 \mathrm{E}, /$,

$842 \mathrm{H} 4 \quad 1-5$ NODEID a node ID number ,/,

$937 \mathrm{H}$

6- 13 NODNAM a node name,/,

$\div 54 \mathrm{H}$

16- 25 YLOC latitude, in decimal degrees,/,

$155 \mathrm{H}$

$26-35$

XLOC

longitude, in decimal degrees ,/,

$273 \mathrm{H}$

$36-40$ NXTNOD

node ID of the downstream node. Mus

$3 t$ be set to ,/,

$431 \mathrm{X}, 39$ Hnegative number for all terminus nodes. , /,

$573 \mathrm{H} \quad 41-50$ PLTX plotting offset from the node symbol

6 , in inches,$/$,

$773 \mathrm{H} 51-60$ PLTY plotting offset from the node symbol

8 , in inches)

WRITE (IOUT, 1020) 
Attachment 7--FORTRAN SOURCE Code of Subroutines for

Streamflow and Water-Quality Simulation--Continued

578

579

580

581

582

583

584

585

586

587

588

589

590

591

592

593

594

595

596

597

598

599

600

601

602

603

604

605

606

607

608

609

610

611

612

613

614

615

616

617

618

619

620

621

622

623

624

625

626

627

628

629

630

631

632

1020 FORMAT $(77 \mathrm{H}$

$61-70$

lmine load routing when $/ /$,

$231 \mathrm{X}, 49 \mathrm{Hincremental}$ streamflow is negative. Value ranges ,/,

$331 \mathrm{X}, 47 \mathrm{Hfrom} 0.0$ to 1.0 , where 0.0 indicates the entire , /,

$431 \mathrm{X}, 49 \mathrm{H}$ load is concentrated downstream and 1.0 indicates ,/,

$531 \mathrm{X}, 43 \mathrm{Hthe}$ concentration is maintained downstream. ,/,

$677 \mathrm{H} \quad 71-80$ DSA

intercept of the water quality-speci

7 fic conductance ,/,

$831 \mathrm{X}, 17 \mathrm{Hlinear}$ regression, /,

$973 \mathrm{H} \quad 81-90$ DSB

*conductance ,/,

$131 \mathrm{X}, 17 \mathrm{Hlinear}$ regression ,/,

$274 \mathrm{H}$ note -- record 5 is repeated 12 times, one for each month, be 3ginning with ,/,

$414 \mathrm{X}, 7 \mathrm{HJ}$ anuary ,/,

$570 \mathrm{H} 511-15$ INDEPV variable number of the independent $v$ 6ariable. ,/,

$731 \mathrm{X}, 49 \mathrm{HIf}$ the value is positive, the incremental flow is ,/,

$836 \mathrm{X}, 31 \mathrm{Hcomputed}$ and routed downstream. ,/,

$931 \mathrm{X}, 48 \mathrm{HIf}$ the value is negative, the total flow at that )

WRITE (IOUT, 1030)

1030 FORMAT (36X,34Hpoint is computed with no routing. ,/,

$131 \mathrm{X}, 42 \mathrm{HIf}$ the value is less than 100 , the flow is,$/$,

$236 \mathrm{X}, 40 \mathrm{Hcomputed}$ using linear regression and the ,/,

3 36X,42Hindependent variable identified by INDEPV. ,/,

$431 \mathrm{X}, 45 \mathrm{HIf}$ the value is greater than 100 , the flow is ,/,

$536 \mathrm{X}, 41 \mathrm{Hcomputed}$ using $\log -10 \mathrm{~g}$ regression and the ,/,

$636 \mathrm{X}, 34 \mathrm{Hindependent}$ variable identified by ,/,

7 36X, 15H (INDEPV - 100). ,/,

$831 \mathrm{X}, 45 \mathrm{HIf}$ the value is greater than 200 , the flow is ,/,

$936 \mathrm{X}, 40 \mathrm{Hcomputed}$ using linear regression and the ,/,

$* 36 \mathrm{X}, 44 \mathrm{Hindependent}$ variable is upstream streamflow. ,/,

$174 \mathrm{H} \quad 16-25$ NTRCPT intercept of the streamflow-independ

2ent variable, /,

$331 \mathrm{X}, 10 \mathrm{Hregression}, /$,

$470 \mathrm{H} \quad 26-35$ SLOPE

5variable, /,

$631 \mathrm{X}, 10 \mathrm{Hregression} \mathrm{,/,}$

$774 \mathrm{H} \quad 36-45$ QWA

8 e-streamflow ,/,

$931 \mathrm{X}, 18 \mathrm{H} \log -\log$ regression )

WRITE (IOUT, 1040)

1040 FORMAT (70H 46- 55 QWB

lance-streamflow,/,

$231 \mathrm{X}, 18 \mathrm{Hlog}-\log$ regression)

CALL QUESZN (ITEMP)

WRITE (IOUT, 1050)

1050 FORMAT (46H The format for the digitized network file is: ,/,

$137 \mathrm{H}$ Record Columns Name Description ,/,

$280 \mathrm{H}+$

3

$476 \mathrm{H} 11-10$ XMIN

$5 \mathrm{~s}$, for mapping, ,/,

$677 \mathrm{H} \quad 11-20$ XMAX

7es, for mapping ,/,

largest longitude, in decimal degree smallest longitude, in decimal degre 
Attachment 7--FORTRAN SOURCE Code of Subroutines for

Streamflow and Water-Quality Simulation--Continued

633

634

635

636

637

638

639

640

641

642

643

644

645

646

647

648

649

650

651

652

653

654

655

656

657

658

659

660

661

662

663

664

665

666

667

668

669

670

671

672

673

674

675

676

677

678

679

680

681

682

683

684

685
$876 \mathrm{H} \quad 21-30$ YMIN

$9 \mathrm{~s}$, for mapping , /,

* 75H 31- 40 YMAX

1 , for mapping ,/,

$276 \mathrm{H} \quad 41-50$ XIENG

3 , on schematic, $/$,

$431 \mathrm{X}, 11$ Hhydrographs ,/,

5 76H $51-60$ YLENG

6 , on schematic ,/,

7 31X, 11Hhydrographs )

WRITE (IOUT, 1060)

1060 FORMAT $(71 \mathrm{H} 221-10 \mathrm{XHY}$ longitude, in decimal degrees

1 , to place first, /,

$231 \mathrm{X}, 20 \mathrm{Hschematic}$ hydrograph ,/,

$380 \mathrm{H} 11-20 \mathrm{YHY}$ latitude, in decimal degrees, to pla 4ce first schematic,/,

$531 \mathrm{X}, 10$ Hhydrograph,$/$,

$672 \mathrm{H} 21-30 \mathrm{XHY}$ longitude, in decimal degrees, to $\mathrm{pl}$

7 ace second,$/$,

$831 \mathrm{X}, 20 \mathrm{Hschematic}$ hydrograph ,/,

$971 \mathrm{H} 31-40 \mathrm{YHY}$ latitude, in decimal degrees, to pla *ce second ,/,

$131 \mathrm{X}, 20 \mathrm{Hschematic}$ hydrograph ,/,

$271 \mathrm{H} \quad 41-50 \mathrm{XHY}$ longitude, in decimal degrees, to $\mathrm{p} 1$ 3ace third,/,

$431 \mathrm{X}, 20 \mathrm{Hschematic}$ hydrograph ,/,

$580 \mathrm{H} 51-60 \mathrm{YHY}$ latitude, in decimal degrees, to pla 6ce third schematic, $/$,

$731 \mathrm{X}, 10$ Hhydrograph)

WRITE (IOUT, 1070)

1070 FORMAT (72H 61- $70 \mathrm{XHY}$ longitude, in decimal degrees

1 , to place fourth ,/,

$231 \mathrm{X}, 20 \mathrm{Hschematic}$ hydrograph ,/,

$371 \mathrm{H} 71-80$ YHY latitude, in decimal degrees, to pla 4ce fourth ,/,

$531 \mathrm{X}, 20 \mathrm{Hs}$ chematic hydrograph ,/,

$671 \mathrm{H} \quad 81-90 \mathrm{XHY}$ longitude, in decimal degrees, to $\mathrm{pl}$

7 ace fifth ,/,

$831 \mathrm{X}, 20 \mathrm{Hschematic}$ hydrograph ,/,

$980 \mathrm{H} 91-100 \mathrm{YHY}$ latitude, in decimal degrees, to pla

*ce fifth schematic,/,

$131 \mathrm{X}$, 10Hhydrograph ,/,

$253 \mathrm{H}$ note -- record 3 is repeated until ICARD is negative ,/,

$369 \mathrm{H} 31-5$ ICARD code indicating disposition of data 4 points. ,/,

$531 \mathrm{X}, 45 \mathrm{HIf}$ value is one, start plotting data in a new ,/,

6 36X, 9Hsequence. ,/,

$731 \mathrm{X}, 45 \mathrm{HIf}$ value is greater than 1, continue plotting ,/,

$836 \mathrm{X}$, 36Hdata in sequence from previous line.)

WRITE (IOUT, 1080)

1080 FORMAT (31X, $32 \mathrm{HIf}$ value is zero, skip the line. ,/,

$131 \mathrm{X}, 48 \mathrm{HI}$ value is negative, quit plotting the network.)

WRITE (IOUT, 1090) 
Attachment 7--FORTRAN SOURCE Code of Subroutines for

Streamflow and Water-Quality Simulation--Continued 1090 FORMAT (66H 6- 12 YLINE latitude, in decimal degrees, 1 of a point ,/,

$267 \mathrm{H}$

3point ,/, $466 \mathrm{H}$

5oint, /, $667 \mathrm{H}$ 7 point ,/, $866 \mathrm{H}$

9oint ,/, $* 67 \mathrm{H}$

1point ,/,

$266 \mathrm{H}$

3oint ,/,

$467 \mathrm{H}$

5point ,/,

$666 \mathrm{H}$

7oint ,/,

$867 \mathrm{H}$

9 point )

CALL QUESZN (ITEMP)

WRITE (IOUT, 1100)

1100 FORMAT ( $49 \mathrm{H}$ The format for the independent variable file is: ,/, $137 \mathrm{H}$ Record Columns Name $280 \mathrm{H}+$

3

$433 \mathrm{H}$

$563 \mathrm{H}$

6),$/$,

$777 \mathrm{H}$

8 (maximum of

$972 \mathrm{H}$

* a re NINDV ,/,

$159 \mathrm{H}$

$224 \mathrm{H}$

$324 \mathrm{H}$

$424 \mathrm{H}$

WRITE (IOUT, 1110)

1110 FORMAT $(24 \mathrm{H}$

$224 \mathrm{H}$

$424 \mathrm{H}$

7 ning in a January ,/,

$876 \mathrm{H} \quad 4 \quad 1-10$

9ent variables. ,/,

$* 75 \mathrm{H}$

11- 20

1 ght per line. ,/,

$270 \mathrm{H}$

21- 30 VARIBL

3ng data),/,

$424 \mathrm{H}$

$524 \mathrm{H}$

$624 \mathrm{H}$

$724 \mathrm{H}$

$824 \mathrm{H}$

C

RETURN

END longitude, in decimal degrees, of a

latitude, in decimal degrees, of a $p$

longitude, in decimal degrees, of a

latitude, in decimal degrees, of a $p$

longitude, in decimal degrees, of a

latitude, in decimal degrees, of a $p$

longitude, in decimal degrees, of a

latitude, in decimal degrees, of a $p$

longitude, in decimal degrees, of a a title, ,/,

the number of months (maximum of 600 the number of independent variables an independent variable name. There occurrences, eight per line.,/,

VARNAM

VARNAM,$/$,

VARNAM , $/$,

VARNAM )

51- 56 VARNAM ,/,

VARNAM , /,

is read as a

VARIBL value of the time series of independ

VARIBL

There are NINDV occurrences, ei (-99. is used to indicate missi 
Attachment 7--FORTRAN SOURCE Code of Subroutines for

Streamflow and Water-Quality Simulation--Continued

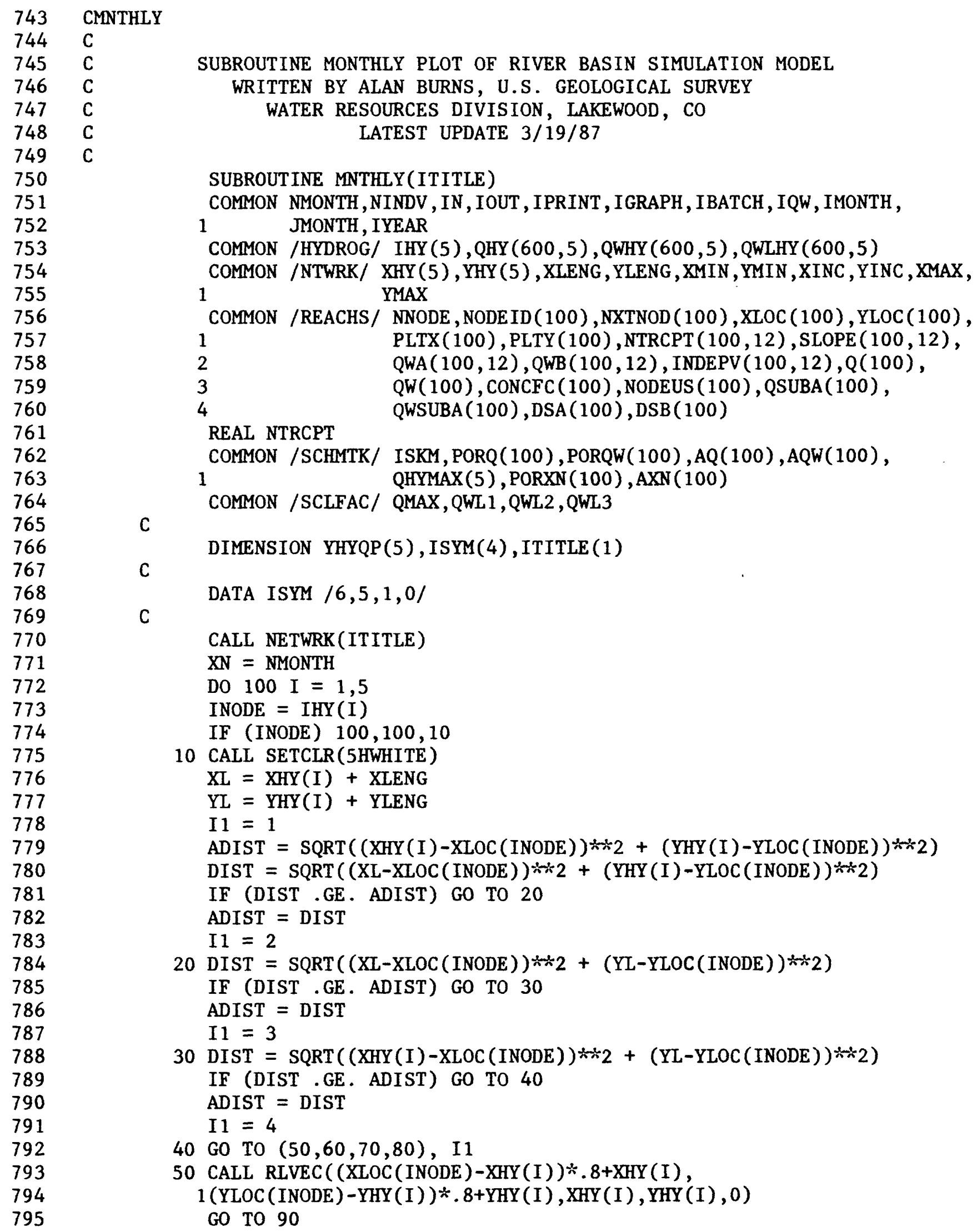


Attachment 7--FORTRAN SOURCE Code of Subroutines for

\section{Streamflow and Water-Quality Simulation--Continued}

60 CALL RLVEC ( $(X L O C(I N O D E)-X L) * .8+X L,(Y L O C($ INODE $)-Y H Y(I)) * .8+Y H Y(I)$, 1XL, YHY (I) , 0) GO TO 90

70 CALL RLVEC ( (XLOC (INODE)-XI)*. 8+XL, (YLOC (INODE) -YL)*. 8+YL, XL, YL , 0) GO TO 90

80 CALL RLVEC ( $(X L O C$ (INODE) -XHY (I) )*.8+XHY (I), (YLOC (INODE) -YL) *. 8+YL, $\operatorname{XXHY}(\mathrm{I}), \mathrm{YL}, 0)$

90 CALL RLVEC (XHY (I), YHY (I), XL, YHY (I) , 0)

CALL RLVEC (XL, YHY (I), XI , YL , 0)

CALL RLVEC (XI, YL, XHY (I), YL , 0)

CALL RLVEC (XHY (I), YL, XHY (I), YHY (I) , 0) $\mathrm{YHYQP}(\mathrm{I})=\mathrm{YHY}(\mathrm{I})$

100 CONTINUE

RETURN

C

ENTRY MONTHA

DO 120 INODE $=1$, NNODE

IPEN $=1$

CALL SETCLR (4HCYAN)

IF (QW(INODE) . LT. QWL1) GO TO 110

IPEN $=2$

CALL SETCLR(7HMAGENTA)

IF (QW(INODE) .LT. QWL2) GO TO 110

IPEN $=3$

CALL SETCLR (6HYELLOW)

IF (QW(INODE) .LT. QWL3) GO TO 110

IPEN $=4$

CALL SETCLR(3HRED)

110 CONTINUE

IF ( $Q$ (INODE) .IE. O.) GO TO 120

CALL MARKER (ISYM(IPEN))

CALL SCLPIC (3.)

CALL CURVE (XLOC (INODE), YLOC (INODE) , $1,-1$ ) C

120 CONTINUE

DO $140 \mathrm{I}=1,5$

INODE $=\mathrm{IHY}(\mathrm{I})$

IF (INODE) $140,140,130$

130 CALL SETCLR(4HCYAN)

IF (QW(INODE) . GT. QWL1) CALL SETCLR(7HMAGENTA)

IF (QW(INODE) .GT. QWL2) CALL SETCLR(6HYELLOW)

IF (QW(INODE) . GT. QWL3) CALL SETCLR(3HRED)

$\mathrm{XL}=\mathrm{XHY}(\mathrm{I})+\mathrm{XIENG} / \mathrm{XN}$

$\mathrm{YL}=\mathrm{YHY}(\mathrm{I})+\mathrm{Q}(\mathrm{INODE}) * \mathrm{YLENG} / \mathrm{QHYMAX}(\mathrm{I})$

CALL RLVEC (XHY (I), YHYQP (I), XI , YL , 0)

$\mathrm{XHY}(\mathrm{I})=\mathrm{XL}$

$\mathrm{YHYQP}(\mathrm{I})=\mathrm{YL}$ 
Attachment 7--FORTRAN SOURCE Code of Subroutines for Streamflow and Water-Quality Simulation--Continued

843

844

845

846

847

848

849

850

851

852

853

854

855

856

857

858
140 CONTINUE

IF (IMONTH .EQ. NMONTH) RETURN

C

DO 150 INODE $=1$, NNODE

IPEN $=1$

IF (QW (INODE) .GT. QWLI) IPEN $=2$

IF (QW(INODE) .GT. QWL2) IPEN $=3$

IF (QW(INODE) .GT. QWL3) IPEN $=4$

CALL SETCLR (5HBLACK)

CALL MARKER(ISYM(IPEN))

CALL SCLPIC (3.)

CALL CURVE (XLOC (INODE), YLOC (INODE) , $1,-1$ )

150 CONTINUE

C

RETURN

END 
Attachment 7--FORTRAN SOURCE Code of Subroutines for

Streamflow and Water-Quality Simulation--Cont inued

859 CNETWRK

$860 \mathrm{C}$

861 C

$862 \quad \mathrm{C}$

$863 \mathrm{C}$

$864 \mathrm{C}$

$865 \mathrm{C}$

SUBROUTINE DRAINAGE NETWORK OF RIVER BASIN SIMULATION MODEL

WRITTEN BY ALAN BURNS, U.S. GEOLOGICAL SURVEY

WATER RESOURCES DIVISION, LAKEWOOD, CO

LATEST UPDATE $9 / 10 / 86$

866

867

868

869

870

871

872

873

874

875

876

877

878

879

880

881

882

883

884

885

886

887

888

889

890

891

892

893

894

895

896

897

898

899

900

901

902

903

904

905

SUBROUTINE NETWRK (ITITLE)

COMMON /NTWRK/ XHY(5), YHY(5), XLENG, YLENG, XMIN , YMIN , XINC , YINC , XMAX, 1 YMAX

C

C

DIMENSION ITITLE (1), XIINE (5), YLINE (5)

C

DATA IBLANK / 1H /

CALL RESET('ALL')

CALL PAGE $(11 ., 8.5)$

CALL HEADIN (ITITLE, 100, 1.43,1)

CALL XNAME $(1 \mathrm{H}, 1)$

CALL YNAME $(1 \mathrm{H}, 1)$

CALL XTICKS $(0)$

CALL YTICKS (0)

CALL XNONUM

CALL YNONUM

REWIND 62

READ $(62,1000)$ XMIN , XMAX, YMIN, YMAX, XLENG, YLENG

READ $(62,1000)$ (XHY (I), YHY (I), I=1,5)

1000 FORMAT (10F10.0)

$\mathrm{XINC}=\mathrm{XMAX}-\mathrm{XMIN}$

YINC $=$ YMAX - YMIN

CALL GRAF (XMIN, XINC, XMAX, YMIN, YINC, YMAX)

CALL SETCLR (4HBLUE)

C

$10 \operatorname{READ}(62,1010) \operatorname{ICARD}$, (YLINE (I1), XIINE (I 1), I 1 $=1,5)$

1010 FORMAT (I5, 10F7.0)

IF (ICARD) $60,10,20$

20 IF (ICARD - 1) $40,40,30$

30 CALL RLVEC (XI, YL, XIINE (1), $\operatorname{YLINE}(1), 0)$

40 DO $50 \mathrm{I1}=2,5$

CALL RLVEC (XLINE (I 1 - 1), YLINE (I 1 - 1), XIINE (I1), YLINE (I1), 0)

50 CONTINUE

$\mathrm{XL}=\mathrm{XLINE}(5)$

$\mathrm{YL}=\mathrm{YLINE}(5)$

GO TO 10

C

60 RETURN

END 
Attachment 7--FORTRAN SOURCE Code of Subroutines for

Streamflow and Water-Quality Simulation--Continued

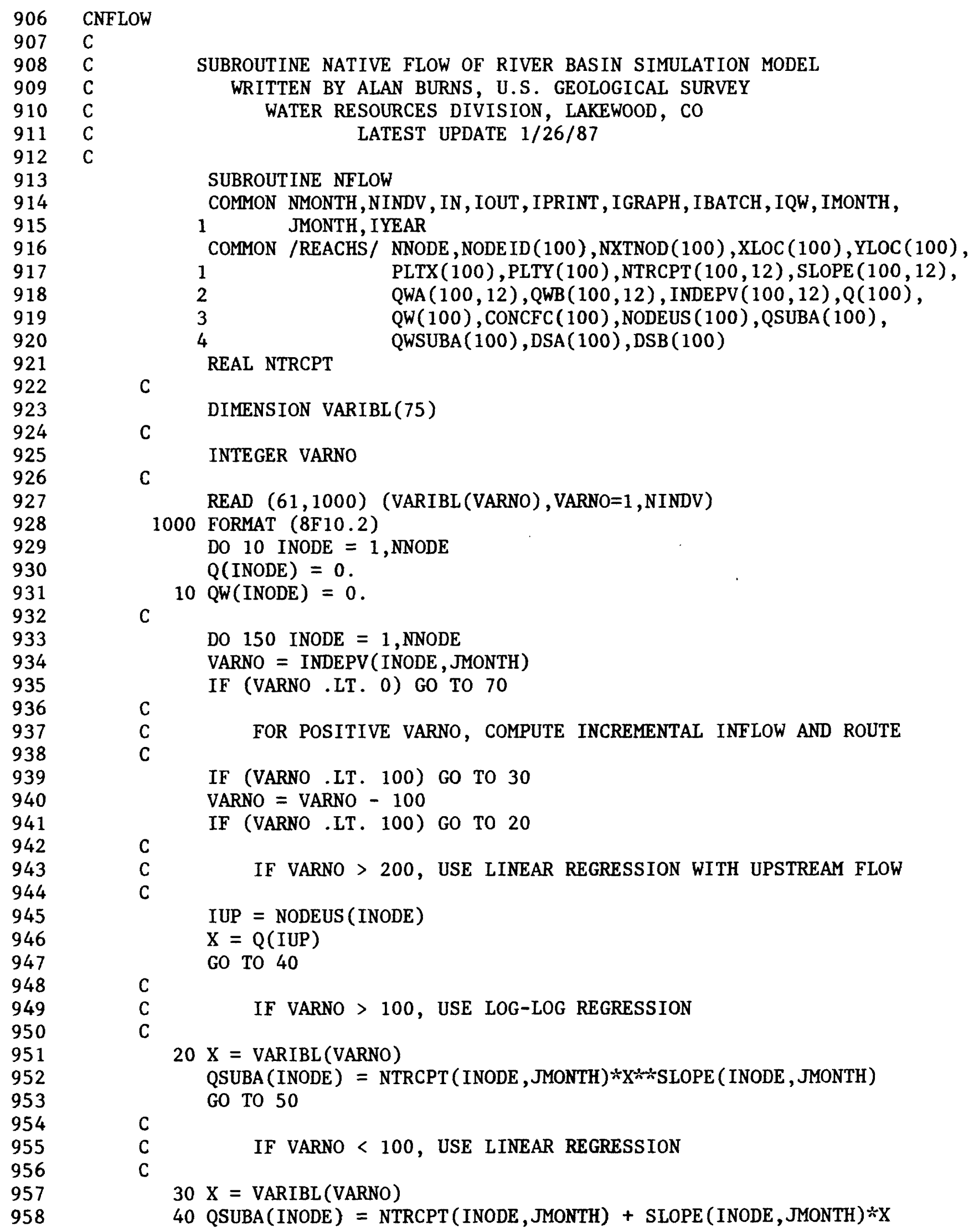

SUBROUTINE NATIVE FLOW OF RIVER BASIN SIMULATION MODEL WRITTEN BY ALAN BURNS, U.S. GEOLOGICAL SURVEY WATER RESOURCES DIVISION, LAKEWOOD, CO LATEST UPDATE $1 / 26 / 87$

COMMON NMONTH, NINDV , IN , IOUT, IPRINT, IGRAPH, IBATCH, IQW, IMONTH, 1 JMONTH, IYEAR

COMMON /REACHS/ NNODE, NODEID (100), NXTNOD (100), XLOC (100), YLOC (100), $1 \quad \operatorname{PLTX}(100), \operatorname{PLTY}(100), \operatorname{NTRCPT}(100,12), \operatorname{SLOPE}(100,12)$, 
Attachment 7--FORTRAN SOURCE Code of Subroutines for

\section{Streamflow and Water-Quality Simulation--Continued}

50 QWSUBA (INODE) $=0$.

IF (QSUBA(INODE) . LE. 0.) GO TO 60

QWSUBA (INODE) $=$ QWA (INODE, JMONTH) *QSUBA (INODE) **QWB (INODE, JMONTH)

QWSUBA (INODE) $=$ DSA (INODE) + DSB (INODE) *QWSUBA (INODE)

$60 \mathrm{QT}=\mathrm{Q}(\mathrm{INODE})+\mathrm{QSUBA}(\mathrm{INODE})$

IF (QT .LE. O.) GO TO 120

$Q W(I N O D E)=(Q W($ INODE $) * Q($ INODE $)+Q W S U B A($ INODE $) * Q S U B A(I N O D E)) / Q T$

IF (QSUBA(INODE) .GE. 0.) GO TO 120

QMIN $=$ QW(INODE) $* Q T / Q($ INODE $)$

$Q W(I N O D E)=Q W(I N O D E)-\operatorname{CONCFC}(I N O D E) *(Q W(I N O D E)-Q M I N)$

GO TO 120

$\mathrm{C}$

$\mathrm{C}$

C

70 VARNO $=$-VARNO

IF (VARNO .LT. 100) GO TO 90

VARNO = VARNO - 100

IF (VARNO . LT. 100) GO TO 80

$\mathrm{C}$

C

$\mathrm{C}$

IF VARNO > 200, USE LINEAR REGRESSION WITH UPSTREAM FLOW
C

C

C

$80 \mathrm{X}=$ VARIBL (VARNO)

IF (X .EQ. -99.) GO TO 130

$Q T=N T R C P T($ INODE, JMONTH) $* X *$ SLOPE (INODE, JMONTH)

GO TO 110

C

C

$\mathrm{C}$

$90 \mathrm{X}=$ VARIBL (VARNO)

IF (X .EQ. -99.) GO TO 130

$100 \mathrm{QT}=\mathrm{NTRCPT}($ INODE, JMONTH$)+$ SLOPE $($ INODE , JMONTH $) * \mathrm{X}$

110 IF (QT .LE. 0.) GO TO 120

$Q W($ INODE $)=$ QWA (INODE, JMONTH) $* Q T * * Q W B$ (INODE, JMONTH)

C

$120 \mathrm{IF}(\mathrm{QT}$.LT. 0.) QT $=0$.

$\mathrm{Q}(\mathrm{INODE})=\mathrm{QT}$

JNODE = NXTNOD (INODE)

IF (JNODE .LT. 0) GO TO 150

$\mathrm{QT}=\mathrm{Q}(\mathrm{INODE})+\mathrm{Q}(\mathrm{JNODE})$

IF (QT .LE. 0.) GO TO 140

$Q W(J N O D E)=(Q W(I N O D E) * Q(I N O D E)+Q W(J N O D E) * Q(J N O D E)) / Q T$

$\mathrm{Q}(\mathrm{JNODE})=\mathrm{QT}$

GO TO 150

$130 \mathrm{Q}($ INODE $)=\mathrm{X}$

$140 \mathrm{QW}(\mathrm{INODE})=-99$.

150 CONTINUE

C

RETURN

END 
Attachment 7--FORTRAN SOURCE Code of Subroutines for

Streamflow and Water-Quality Simulation--Continued

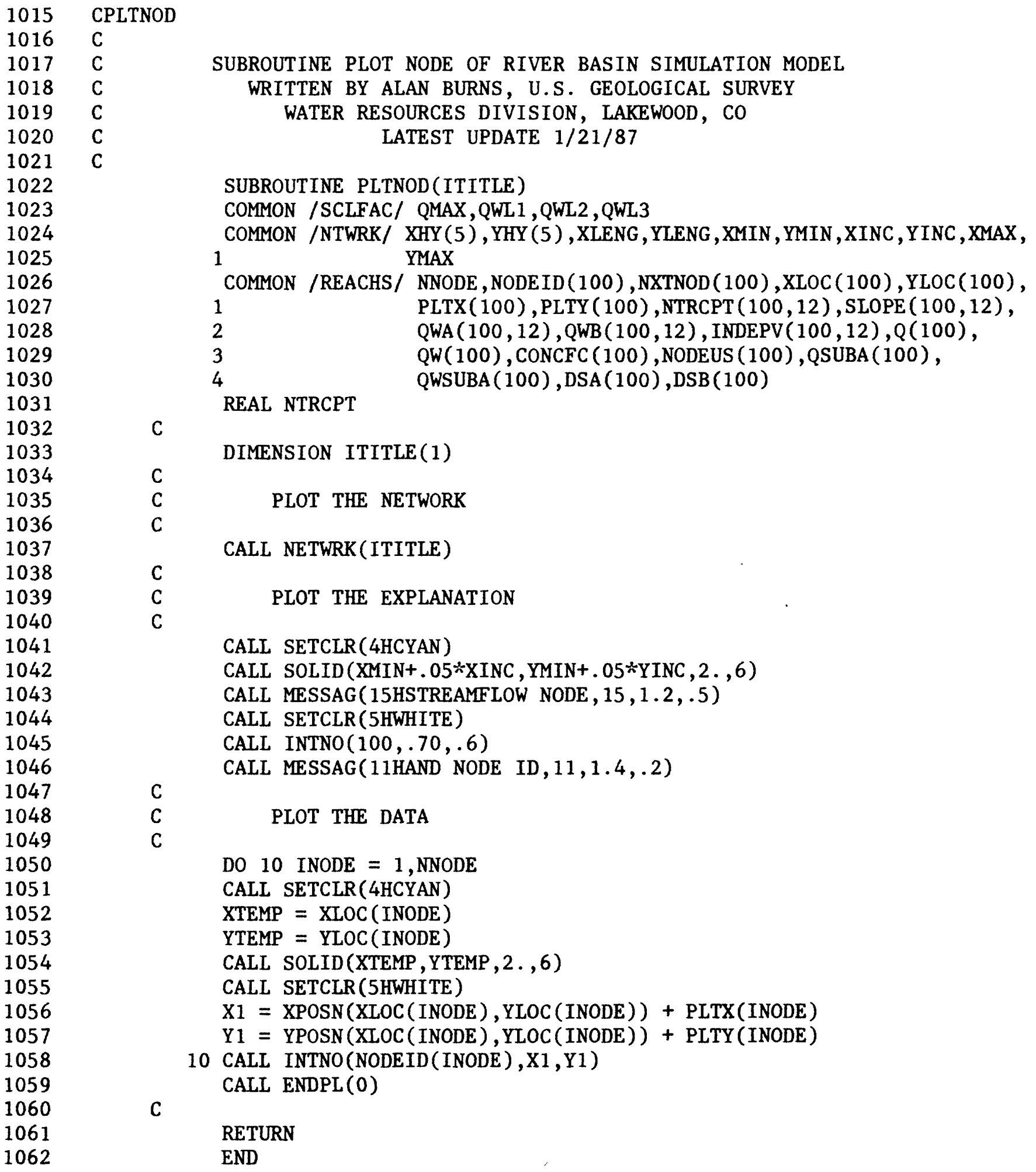


Attachment 7--FORTRAN SOURCE Code of Subroutines for Streamflow and Water-Quality Simulation--Continued

1063

1064

1065

1066

1067

1068

1069

1070

1071

1072

1073
SUBROUTINE PROB(ARRAY,NPTS)

C

C

DIMENSION ARRAY(1)

$\mathrm{FAC}=$ NPTS

$\mathrm{FAC}=1 . /(\mathrm{FAC}+1$.

DO 10 IX $=1$, NPTS

$\mathrm{XI}=\mathrm{IX}$

$10 \operatorname{ARRAY}(I X)=$ FUNCZ $(X I * F A C)$

RETURN

END 
Attachment 7--FORTRAN SOURCE Code of Subroutines for

Streamflow and Water-Quality Simulation--Continued

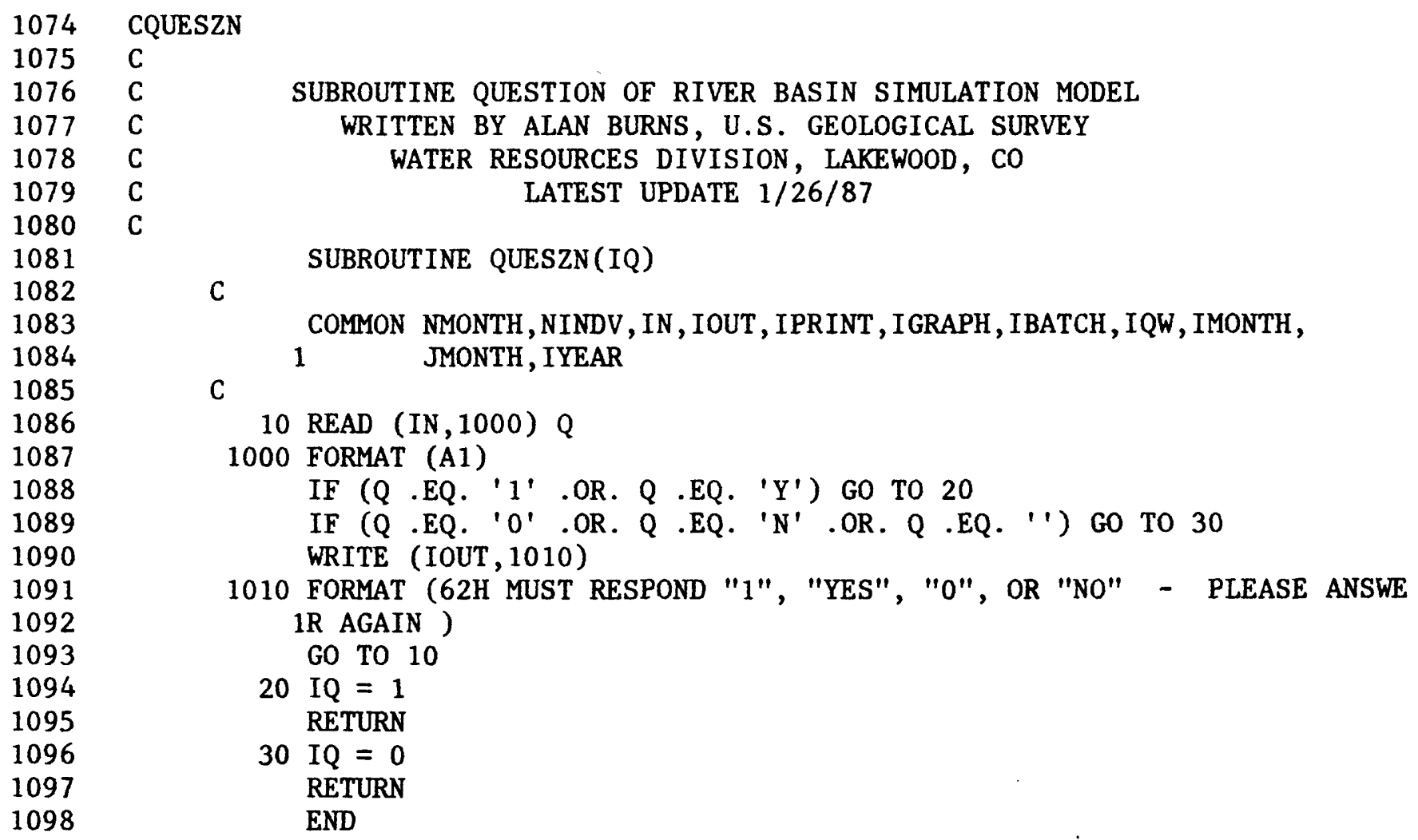

SUBROUTINE QUESTION OF RIVER BASIN SIMULATION MODEL

WRITTEN BY ALAN BURNS, U.S. GEOLOGICAL SURVEY

WATER RESOURCES DIVISION, LAKEWOOD, CO

LATEST UPDATE $1 / 26 / 87$

C

SUBROUTINE QUESZN(IQ)

COMMON NMONTH, NINDV , IN , IOUT, IPRINT , I GRAPH, IBATCH, IQW, IMONTH, 
Attachment 7--FORTRAN SOURCE Code of Subroutines for

Streamflow and Water-Quality Simulation--Continued

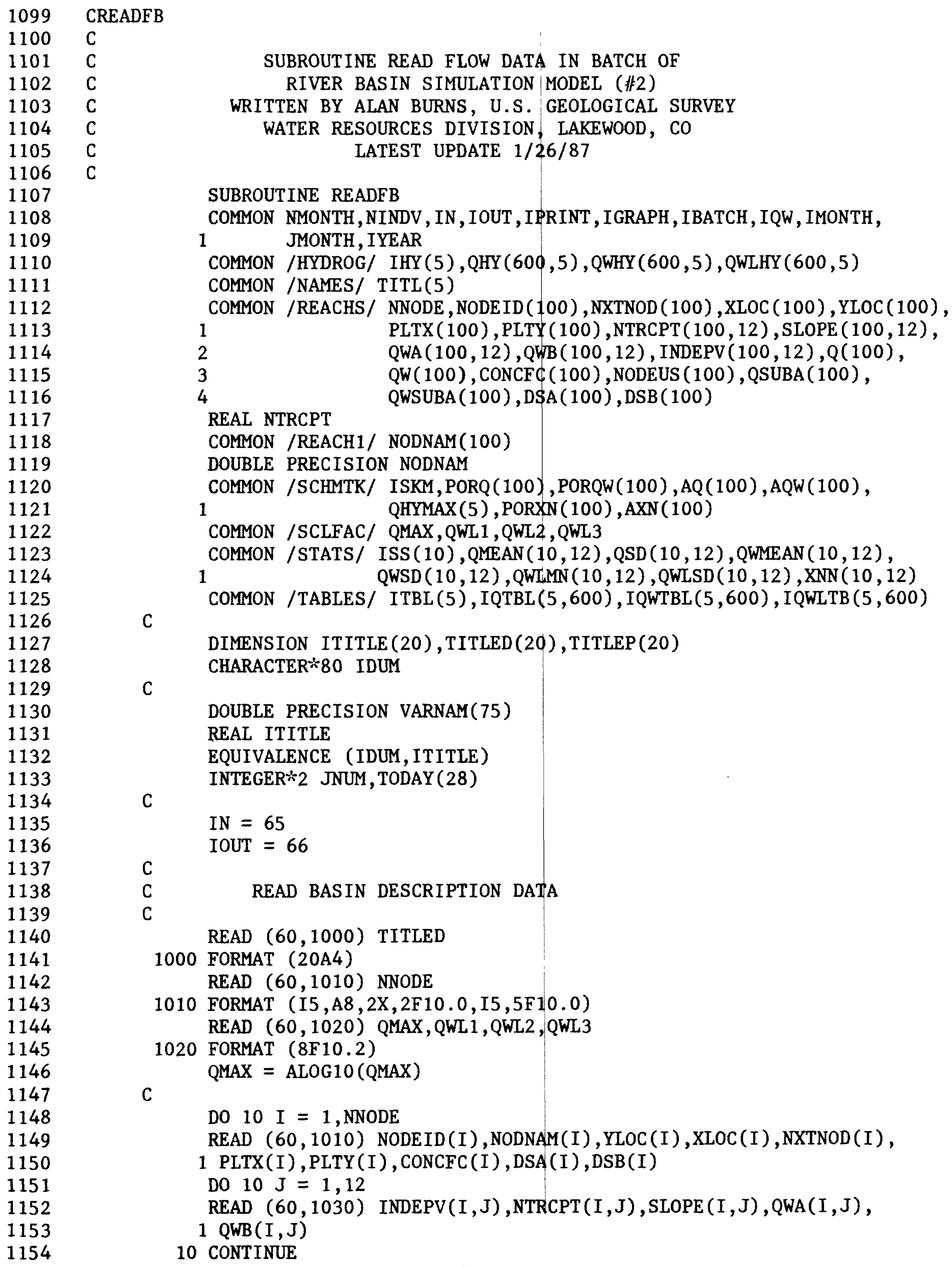

SUBROUTINE READ FLOW DATA IN BATCH OF RIVER BASIN SIMULATION MODEL (非) WRITTEN BY ALAN BURNS, U.S. GEOLOGICAL SURVEY WATER RESOURCES DIVISION, LAKEWOOD, CO LATEST UPDATE $1 / 26 / 87$ 
Attachment 7--FORTRAN SOURCE Code of Subroutines for

Streamflow and Water-Quality Simulation--Continued

1155

1156

1157

1158

1159

1160

1161

1162

1163

1164

1165

1166

1167

1168

1169

1170

1171

1172

1173

1174

1175

1176

1177

1178

1179

1180

1181

1182

1183

1184

1185

1186

1187

1188

1189

1190

1191

1192

1193

1194

1195

1196

1197

1198

1199

1200

1201

1202

1203

1204

1205

1206

1207

1030 FORMAT (10X, I5, 4F 10.0)

C

NNODE $1=$ NNODE -1

DO $30 \mathrm{I}=1$, NNODE 1

DO $20 \mathrm{~J}=1$, NNODE

IF (NXTNOD(I) .NE. NODEID(J)) GO TO 20

$\operatorname{NXTNOD}(I)=\mathrm{J}$

GO TO 30

20 CONTINUE

30 CONTINUE

C

C

C

COMPUTE THE ARRAY OF UPSTREAM NODES

DO 40 INODE $=1$, NNODE

40 NODEUS (INODE) $=0$

DO 50 INODE $=1$, NNODE

NEXT $=$ NXTNOD (INODE)

IF (NODEUS (NEXT) .NE. 0) GO TO 50

NODEUS $($ NEXT) $=$ INODE

50 CONTINUE

C

$\mathrm{C}$

C

READ INDEPENDENT VARIABLE INFORMATION

READ $(61,1000)$ TITLEP

READ $(61,1040)$ NMONTH, NINDV

1040 FORMAT (16I5)

$\operatorname{READ}(61,1050)$ (VARNAM(J), J=1, NINDV)

1050 FORMAT $(8(\mathrm{~A} 6,4 \mathrm{X}))$

C

C ENTER TITLE

$\mathrm{C}$

READ (IN, 1000) TITL

NNUM $=28$

CALL TIMDAT (TODAY, JNUM)

C

C WRITE BASIN DESCRIPTION DATA

C

WRITE (IOUT, 1060) TITL, (TODAY (JNUM), JNUM=1, 3)

1060 FORMAT (20H SIMULATION TITLE: ,5A4,23X, 10HDATE RUN: ,A2, 1H/,A2, $1 \mathrm{HH} /, \mathrm{A} 2)$

WRITE (IOUT, 1070) TITLED

1070 FORMAT (//44H THE BASIN DESCRIPTION FILE YOU ATTACHED IS: ,/,20A4)

WRITE (IOUT, 1080) TITLEP, NMONTH, NINDV

1080 FORMAT (//47H THE INDEPENDENT VARIABLE FILE YOU ATTACHED IS:,/, $120 \mathrm{~A} 4, /, 10 \mathrm{X}, 19 \mathrm{HTHAT}$ FILE CONTAINS , I4,22H MONTHS OF RECORD FOR ,

2 I3, 10H VARIABLES, //)

C

WRITE $(63,1090)$ TITL, (TODAY (JNUM), JNUM=1,3), TITLED, NNODE

1090 FORMAT (82H1 BSNMD2 - RIVER BASIN SIMULATION MODEL 非2

1D $9 / 10 / 86$ BY ALAN BURNS,$/ / /, 20 \mathrm{H}$ SIMULATION TITLE: ,5A4, $266 \mathrm{X}, 10 \mathrm{H}$ DATE RUN: ,A2,1H/,A2,1H/,A2,///, 326H BASIN DESCRIPTION FILE: ,20A4, 10H CONTAINS , I3,6H NODES ) WRITE $(63,1100)$ TITLEP, NINDV, NMONTH 
Attachment 7--FORTRAN SOURCE Code of Subroutines for

Streamflow and Water-Quality Simulation--Continued

1208

1209

1210

1211

1212

1213

1214

1215

1216

1217

1218

1219

1220

1221

1222

1223

1224

1225

1226

1227

1228

1229

1230

1231

1232

1233

1234

1235

1236

1237

1238

1239

1240

1241

1242

1243

1244

1245

1246

1247

1248

1249

1250

1251

1252

1253

1254

1255

1256

1257

1258

1259

1260

1261

1262

1100 FORMAT ( $/ /, 29 \mathrm{H}$ INDEPENDENT VARIABLE FILE: ,20A4,/,10X,8HCONTAINS $1,13,13 H$ RECORDS FOR , I3,7H MONTHS )

WRITE $(63,1110)$ (I , NODEID(I), NODNAM(I), XLOC (I), YLOC (I) , NXTNOD(I), $1 \operatorname{CONCFC}(I), \operatorname{DSA}(I), \operatorname{DSB}(I), I=1, \mathrm{NNODE})$

1110 FORMAT $(/ /, 72 \mathrm{H}$ NODE NODE NODE $X \quad Y$ NEXT CONCEN 1TRATION TDS / SC , /,77H NUMBER ID NAME COORD COORD 2 NODE FACTOR INTERCEPT SLOPE ,//, (I5 , I8, 2X, A8, 2F7.2 , I6, 3 F9.2,F13.2,F9.2)) DO 110 NOD $=1$, NNODE WRITE $(63,1120)$ NODEID(NOD), NODNAM(NOD)

1120 FORMAT (/,32H REGRESSION PARAMETERS FOR NODE , I5,2H (, A8, 1H),/, $176 \mathrm{H}$ MONTH VARIABLE VARIABLE TYPE OF 2WATER QUALITY ,/,77H NUMBER ID RELATIONSHIP INT 3ERCEPT SLOPE INTERCEPT SLOPE ,/) DO $110 \mathrm{~J}=1,12$

IND $=\operatorname{INDEPV}(N O D, J)$

IF (IND .LT. 0) GO TO 80

IF (IND .GT. 100) GO TO 60

WRITE $(63,1130) \mathrm{J}$, INDEPV(NOD, J), VARNAM (IND), NTRCPT (NOD , J), 1 SLOPE (NOD, J), QWA (NOD, J), QWB (NOD, J)

1130 FORMAT (I5, I8,6X, A6, 4X, 10HINC-LINEAR, F 10.3,3F9.3) GO TO 110

60 IND $=$ IND -100

IF (IND . GT. 100) GO TO 70

WRITE $(63,1140) \mathrm{J}, \operatorname{INDEPV}(\mathrm{NOD}, \mathrm{J})$, VARNAM(IND) , NTRCPT (NOD , J) , 1 SLOPE (NOD, J), QWA (NOD , J), QWB (NOD, J)

1140 FORMAT (I5, I8,6X, A6, 4X, 10HINC-LOGLOG,F10.3,3F9.3) GO TO 110

70 WRITE $(63,1150) \mathrm{J}, \operatorname{INDEPV}(\mathrm{NOD}, \mathrm{J}), \operatorname{NTRCPT}(\mathrm{NOD}, J)$, SLOPE (NOD , J), 1 QWA(NOD, J), QWB (NOD, J)

1150 FORMAT (I5, I8, 6X, 10HUPSTREAM , 10HINC-IINEAR, F10.3,3F9.3) GO TO 110

$80 \mathrm{IND}=-\mathrm{IND}$ IF (IND . GT. 100) GO TO 90

WRITE $(63,1160) \mathrm{J}, \operatorname{INDEPV}(N O D, J), \operatorname{VARNAM}$ ( IND) , NTRCPT (NOD , J) , 1 SLOPE (NOD, J), QWA (NOD , J), QWB (NOD , J)

1160 FORMAT (I5 , I 8, 6X, A6, 4X, 10HTOT-LINEAR, F10.3 , 3F9.3) GO TO 110

90 IND = IND - 100

IF (IND . GT. 100) GO TO 100

WRITE $(63,1170) \mathrm{J}, \operatorname{INDEPV}(\mathrm{NOD}, \mathrm{J})$, VARNAM (IND) , NTRCPT (NOD , J),

1 SLOPE (NOD , J), QWA (NOD , J), QWB (NOD , J)

1170 FORMAT (I5, I8,6X, A6, 4X, 10HTOT-LOGLOG, F10.3, 3F9.3) GO TO 110

100 WRITE $(63,1180) \mathrm{J}, \operatorname{INDEPV}($ NOD , J ), NTRCPT (NOD , J) , SLOPE (NOD , J) ,

1 QWA(NOD, J), QWB (NOD , J)

1180 FORMAT (I5, I8, 6X, 10HUPSTREAM , 10HTOT-LINEAR,F10.3,3F9.3)

110 CONTINUE

C

CALL QUESZN (IGRAPH)

IF (IGRAPH .NE. 0) CALL COMPRS

C

C

ENTER FREQUENCY OF PLOTTING SCHEMATIC

READ (IN, 1040) ISKM 
Attachment 7--FORTRAN SOURCE Code of Subroutines for Streamflow and Water-Quality Simulation--Continued

1263

1264

1265

1266

1267

1268

1269

1270

1271

1272

1273

1274

1275

1276

1277

1278

1279

1280

1281

1282

1283

1284

1285

1286

1287

1288

1289

1290

1291

1292

1293

1294

1295

1296

1297

1298

1299

1300

1301

1302

1303

1304

1305

1306

1307

1308

1309

1310

1311

1312

1313

1314

1315

1316

1317

1318
C

C ENTER NODES FOR HYDROGRAPHS

C

READ (IN , 1040) IHY

READ (IN, 1020) QHYMAX

DO $130 \mathrm{I}=1,5$

DO $120 \mathrm{~J}=1$, NNODE

IF (IHY(I) .NE. NODEID(J)) GO TO 120

$\mathrm{IHY}(\mathrm{I})=\mathrm{J}$

GO TO 130

120 CONTINUE

C

130 CONTINUE

C ENTER NODES FOR MONTHLY TABLES

C

READ (IN, 1040) ITBL

DO $150 \mathrm{I}=1,5$

DO $140 \mathrm{~J}=1$, NNODE

IF (ITBL(I) .NE. NODEID(J)) GO TO 140

$\operatorname{ITBL}(\mathrm{I})=\mathrm{J}$

GO TO 150

140 CONTINUE

150 CONTINUE

C

C ENTER NODES FOR STATISTICAL SUMMARIES

C

READ (IN, 1040) ISS

DO $180 \mathrm{I}=1,10$

IF (ISS(I) .LE. 0) GO TO 180

DO $160 \mathrm{~J}=1,12$

$\operatorname{XNN}(I, J)=0$.

$\operatorname{QMEAN}(I, J)=0$.

$\operatorname{QSD}(I, J)=0$.

$\operatorname{QWMEAN}(I, J)=0$.

$\operatorname{QWSD}(I, J)=0$.

$\operatorname{QWLMN}(I, J)=0$.

$160 \operatorname{QWLSD}(\mathrm{I}, \mathrm{J})=0$.

DO $170 \mathrm{~J}=1$, NNODE

IF (ISS(I) .NE. NODEID(J)) GO TO 170

$\operatorname{ISS}(I)=J$

GO TO 180

170 CONTINUE

180 CONTINUE

C

C PLOT NODE LOCATIONS

C

CALL QUESZN (ILGND)

IF (ILGND - 1) $200,190,200$

190 WRITE (IDUM, 1190) TITL

1190 FORMAT (5A4,18H - NODE LOCATIONS\$)

CALL PLTNOD (ITITLE)

C

200 CALL QUESZN (IQW)

IPRINT $=0$

RETURN

END 
Attachment 7--FORTRAN SOURCE Code of Subroutines for

Streamflow and Water-Quality Simulation--Continued

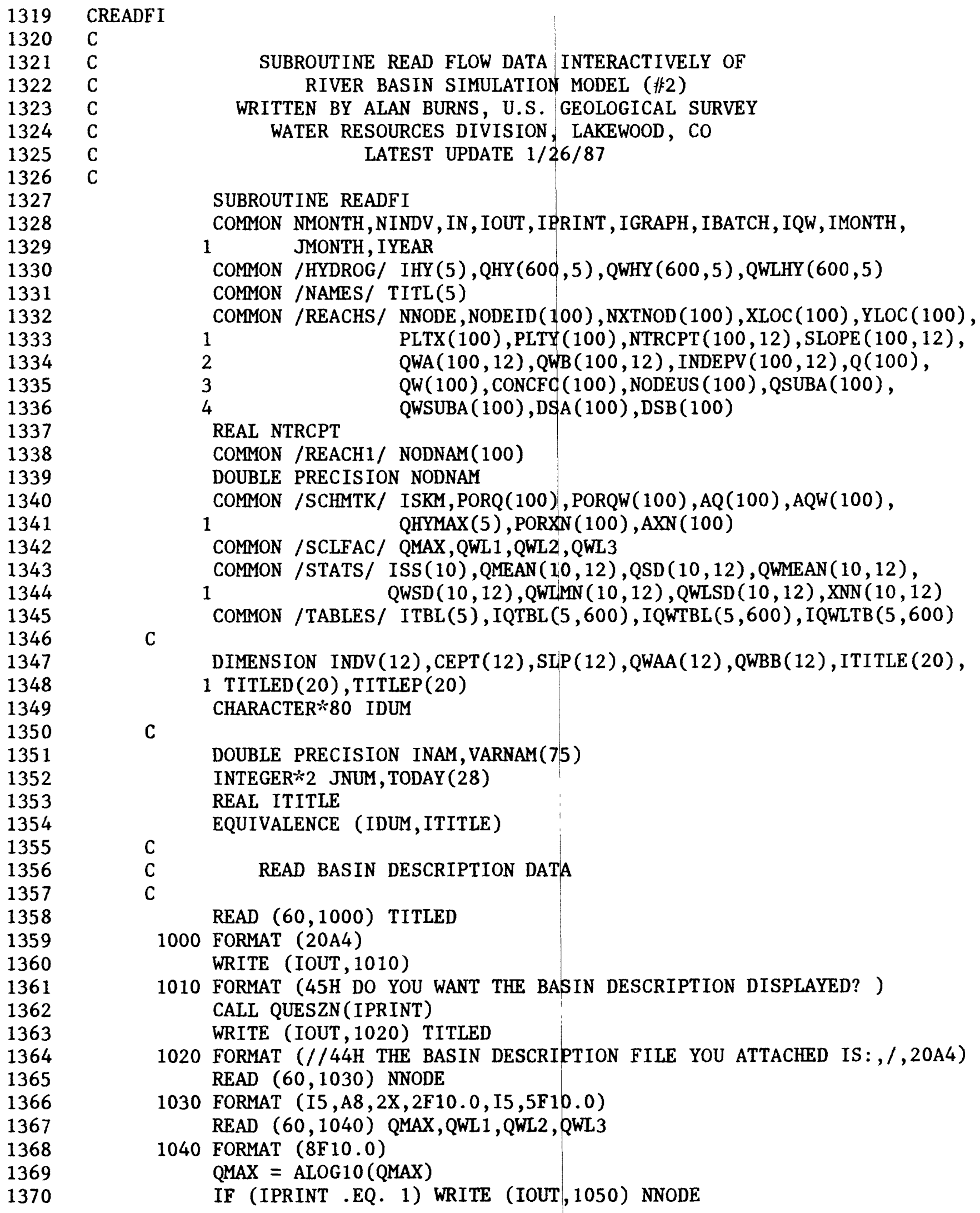

SUBROUTINE READ FLOW DATA INTERACTIVELY OF

RIVER BASIN SIMULATION MODEL (非)

WRITTEN BY ALAN BURNS, U.S. GEOLOGICAL SURVEY WATER RESOURCES DIVISION, LAKEWOOD, CO LATEST UPDATE $1 / 26 / 87$

SUBROUTINE READFI

COMMON NMONTH, NINDV , IN, IOUT, IPRINT , IGRAPH, IBATCH, IQW, IMONTH, 1 JMONTH, IYEAR

COMMON /HYDROG/ IHY (5), QHY $(600,5)$, QWHY $(600,5)$, QWLHY $(600,5)$ COMMON /NAMES/ TITL(5)

COMMON /REACHS/ NNODE, NODEID(100), NXTNOD(100), XLOC (100), YLOC (100), 1

2

3

4

REAL NTRCPT $\operatorname{PLTX}(100), \operatorname{PLTY}(100), \operatorname{NTRCPT}(100,12), \operatorname{SLOPE}(100,12)$, QWA $(100,12), Q W B(100,12), \operatorname{INDEPV}(100,12), Q(100)$, QW(100), CONCFC (100), NODEUS (100), QSUBA (100), QWSUBA (100), DSA(100), DSB(100)

\section{COMMON /REACH1/ NODNAM(100)}

DOUBLE PRECISION NODNAM

COMMON /SCHMTK/ ISKM,PORQ(100), PORQW(100), AQ(100), AQW(100), 1

COMMON /SCLFAC/ QMAX,QWL1, QWL2,QWL3

COMMON /STATS/ ISS $(10), \operatorname{QMEAN}(10,12), \operatorname{QSD}(10,12), \operatorname{QWMEAN}(10,12)$, 1 $\operatorname{QWSD}(10,12), \operatorname{QWLMN}(10,12), \operatorname{QWLSD}(10,12), \operatorname{XNN}(10,12)$

$\mathrm{C}$ COMMON /TABLES/ ITBL(5), IQTBL $(5,600), \operatorname{IQWTBL}(5,600), \operatorname{IQWLTB}(5,600)$

DIMENSION INDV (12), CEPT (12), SLP (12), QWAA (12), QWBB (12), ITITLE (20), $1 \operatorname{TITLED}(20), \operatorname{TITLEP}(20)$ CHARACTER $* 80$ IDUM

C

DOUBLE PRECISION INAM, VARNAM(75)

INTEGER $* 2$ JNUM, TODAY (28)

REAL ITITLE

EQUIVALENCE (IDUM, ITITLE)

$\mathrm{C}$

$\mathrm{C}$

$\mathrm{C}$

1000 FORMAT (20A4)

WRITE (IOUT, 1010)

1010 FORMAT (45H DO YOU WANT THE BASIN DESCRIPTION DISPLAYED? ) CALI QUESZN (IPRINT)

WRITE (IOUT, 1020) TITLED

1020 FORMAT (//44H THE BASIN DESCRIPTION FILE YOU ATTACHED IS: ,/,20A4) READ $(60,1030)$ NNODE

1030 FORMAT (I5, A8, 2X, 2F $10.0, \mathrm{I} 5,5 \mathrm{~F} 10.0)$

READ $(60,1040)$ QMAX, QWL1, QWL2, QWL3

1040 FORMAT $(8 \mathrm{~F} 10.0)$

QMAX = ALOG10 (QMAX)

IF (IPRINT .EQ. 1) WRITE (IOUT, 1050) NNODE 
Attachment 7--FORTRAN SOURCE Code of Subroutines for

Streamflow and Water-Quality Simulation--Continued

1050 FORMAT $(/ /, 11 \mathrm{H}$ THERE ARE , I3,19H NODES IN THE BASIN ) C

DO $10 \mathrm{I}=1$, NNODE

READ $(60,1030)$ NODE ID (I), NODNAM(I), YLOC (I), XLOC (I), NXTNOD (I), $1 \operatorname{PLTX}(I), \operatorname{PLTY}(I), \operatorname{CONCFC}(\mathrm{I}), \mathrm{DSA}(\mathrm{I}), \mathrm{DSB}(\mathrm{I})$

DO $10 \mathrm{~J}=1,12$

$\operatorname{READ}(60,1060) \operatorname{INDEPV}(I, J), \operatorname{NTRCPT}(I, J), \operatorname{SLOPE}(I, J), Q W A(I, J)$, $1 \operatorname{QWB}(I, J)$

10 CONTINUE

1060 FORMAT (10X, I5 , 4F 10.0$)$

C

NNODE $1=$ NNODE -1

DO $30 I=1$, NNODE 1

DO $20 \mathrm{~J}=1$, NNODE

IF (NXTNOD(I) .NE. NODEID(J)) GO TO 20

$\operatorname{NXTNOD}(I)=J$

GO TO 30

20 CONTINUE

30 CONTINUE

C

$\mathrm{C}$

C

READ INDEPENDENT VARIABLE INFORMATION

READ $(61,1000)$ TITLEP

READ $(61,1070)$ NMONTH, NINDV

1070 FORMAT (16I5)

$\operatorname{READ}(61,1080)$ (VARNAM(J), J=1,NINDV)

1080 FORMAT $(8(\mathrm{~A} 6,4 \mathrm{X}))$

$\mathrm{C}$

C

DISPLAY BASIN DESCRIPTION DATA

C

IF (IPRINT .NE. 1) GO TO 110

WRITE (IOUT, 1090) (I ,NODEID (I), NODNAM(I), XLOC (I), YLOC (I), $1 \operatorname{NXTNOD}(I), \operatorname{CONCFC}(I), \operatorname{DSA}(I), \operatorname{DSB}(I), I=1, N N O D E)$

1090 FORMAT $(/ /, 72 \mathrm{H}$ NODE NODE NODE $\mathrm{X}$ Y NEXT CONCEN 1TRATION TDS / SC ,/,77H NUMBER ID NAME COORD COORD 2 NODE FACTOR INTERCEPT SLOPE ,//, (I5 , I 8, 2X , A8, 2F 7.2 , I6, 3 F9.2,F13.2,F9.2))

40 WRITE (IOUT, 1100)

1100 FORMAT (/,78H ENTER THE NUMBER OF THE NODE TO DISPLAY ITS REGRESSI ION PARAMETERS ( 5 COLUMNS) )

READ (IN, 1030) NOD

IF (NOD .LE. 0 .OR. NOD .GT. NNODE) GO TO 110

WRITE (IOUT , 1110) NODE ID (NOD), NODNAM(NOD)

1110 FORMAT (/,32H REGRESSION PARAMETERS FOR NODE , I5,2H (,A8,1H),/, $176 \mathrm{H}$ MONTH VARIABLE VARIABLE TYPE OF $\begin{array}{lrlr}\text { 2WATER QUALITY } & , /, 77 \mathrm{H} & \text { NUMBER } \\ \text { 3ERCEPT } & \text { SLOPE } & \text { INTERCEPT } & \text { SLOPE ,/) }\end{array}$ STREAMFLOW

DO $100 \mathrm{~J}=1,12$

IND $=\operatorname{INDEPV}($ NOD,$J)$

IF (IND .LT. 0) GO TO 70

IF (IND .GT. 100) GO TO 50

WRITE (IOUT, 1120) J, INDEPV(NOD , J), VARNAM(IND), NTRCPT (NOD , J), 1 SLOPE (NOD, J), QWA(NOD , J), QWB (NOD , J) 
Attachment 7--FORTRAN SOURCE Code of Subroutines for

Streamflow and Water-Quality Simulation--Continued

1424

1425

1426

1427

1428

1429

1430

1431

1432

1433

1434

1435

1436

1437

1438

1439

1440

1441

1442

1443

1444

1445

1446

1447

1448

1449

1450

1451

1452

1453

1454

1455

1456

1457

1458

1459

1460

1461

1462

1463

1464

1465

1466

1467

1468

1469

1470

1471

1472

1473

1474

1120 FORMAT (I5, I8, 6X, A6, 4X, 10HINC-LINEAR, F 10.3, 3F9.3)

GO TO 100

50 IND $=$ IND -100

IF (IND . GT . 100) GO TO 60

WRITE (IOUT, 1130) J, INDEPV (NOD, J), VARNAM (IND), NTRCPT (NOD,J),

$1 \operatorname{SLOPE}($ NOD , J), QWA (NOD , J), QWB (NOD, J)

1130 FORMAT (I5, I8, 6X, A6, 4X, 10HINC-LOGLOG, F 10.3, 3F9.3)

GO TO 100

60 WRITE (IOUT, 1140) J, INDEPV(NOD, J), NTRCPT (NOD, J), SLOPE (NOD, J),

1 QWA (NOD, J) , QWB (NOD, J)

1140 FORMAT (I5, I8, 6X, 10HUPSTREAM , 10HINC-LINEAR, F 10.3 , 3F9.3) GO TO 100

70 IND $=-$ IND

IF (IND .GT. 100) GO TO 80

WRITE (IOUT, 1150) J, INDEPV (NOD, J), VARNAM (IND) , NTRCPT (NOD, J),

1 SLOPE (NOD, J), QWA (NOD , J) , QWB (NOD, J)

1150 FORMAT (I5, I8,6X, A6, 4X, 10HTOT-LINEAR, F 10.3, 3F9.3)

GO TO 100

80 IND $=$ IND -100

IF (IND .GT. 100) GO TO 90

WRITE (IOUT, 1160) J, INDEPV (NOD, J), VARNAM (IND), NTRCPT (NOD, J),

1 SLOPE (NOD, J), QWA(NOD, J), QWB (NOD, J)

1160 FORMAT (I5, I8, 6X A6, 4X, 10HTOT-LOGLOG , F 10.3 ,3F9.3)

GO TO 100

90 WRITE (IOUT, 1170) J, INDEPV (NOD, J), NTRCPT (NOD, J), SLOPE (NOD , J),

1 QWA(NOD, J), QWB (NOD, J)

1170 FORMAT (I5, I8, 6X, 10HUPSTREAM, 10HTOT-LINEAR, F 10.3, 3F9.3)

100 CONTINUE

GO TO 40

C

$\mathrm{C}$

$\mathrm{C}$

110 WRITE (IOUT, 1180) TITLEP, NMONTH,NINDV

1180 FORMAT (//47H THE INDEPENDENT VARIABLE FILE YOU ATTACHED IS : , /,

1 20A4,/,10X,19HTHAT FILE CONTAINS , I4,22H MONTHS OF RECORD FOR,

2 I3, 10H VARIABLES, //)

$\mathrm{C}$

$\mathrm{C}$

$\mathrm{C}$

120 WRITE (IOUT, 1190)

1190 FORMAT (45H DO YOU WANT TO MODIFY THE BASIN DESCRIPTION? )

CALL QUESZN(IQ)

IF (IQ - 1) $310,130,310$

130 WRITE (IOUT, 1200)

1200 FORMAT (27H DO YOU WANT TO ADD A NODE? )

CALL QUESZN(IQ)

IF (IQ - 1) $260,140,260$

C

C ADD A NEW NODE

$\mathrm{C}$

140 WRITE (IOUT, 1210) 
Attachment 7--FORTRAN SOURCE Code of Subroutines for

Streamflow and Water-Quality Simulation--Continued

1475

1476

1477

1478

1479

1480

1481

1482

1483

1484

1485

1486

1487

1488

1489

1490

1491

1492

1493

1494

1495

1496

1497

1498

1499

1500

1501

1502

1503

1504

1505

1506

1507

1508

1509

1510

1511

1512

1513

1514

1515

1516

1517

1518

1519

1520

1521

1522

1523

1524

1525

1210 FORMAT (78H ENTER THE NODE ID OF THE NODE UPSTREAM OF THIS NEW NOD 1E OR THE NEGATIVE NODE , /, 76H ID OF THE DOWNSTREAM NODE IF TH 2IS NEW NODE IS A HEADWATER NODE (5 COLS) )

READ (IN, 1070) IADD

IF (IADD .LT. 0) GO TO 160

DO 150 INOD $=1$, NNODE

IF (IADD .EQ. NODEID(INOD)) GO TO 180

150 CONTINUE

WRITE (IOUT, 1220)

1220 FORMAT (26HTHAT NODE ID WAS NOT FOUND )

GO TO 140

160 DO 170 INOD $=1$, NNODE

IF (-IADD .EQ. NODEID(INOD)) GO TO 190

170 CONTINUE

WRITE (IOUT, 1220)

GO TO 140

180 INOD $=$ INOD +1

190 WRITE (IOUT, 1230)

1230 FORMAT (28H ENTER THE NODE ID (5 COLS) )

READ (IN, 1070) IADD

WRITE (IOUT, 1240)

1240 FORMAT (30H ENTER THE NODE NAME ( 8 COLS) )

READ (IN , 1250) INAM

1250 FORMAT (A8)

WRITE (IOUT, 1260) XLOC (INOD)

1260 FORMAT (46H ENTER THE X-COORDINATE (10 COLS) OR 〈CR> IF ,F $10.4,14$

$1 \mathrm{H}$ IS ACCEPTABLE )

READ (IN, 1040) XI

IF (XL .EQ. O.) XL = XIOC (INOD)

WRITE (IOUT, 1270) YLOC (INOD)

1270 FORMAT (46H ENTER THE Y-COORDINATE (10 COLS) OR 〈CR> IF ,F 10.4, 14

$1 H$ IS ACCEPTABLE )

READ (IN, 1040) YL

IF (YL .EQ. 0.) YL = YLOC (INOD)

NXT $=$ NXTNOD $($ INOD-1)

WRITE (IOUT, 1280) NODEID(NXT)

1280 FORMAT (62H ENTER THE NODE ID OF THE DOWNSTREAM NODE (5 COLS) OR $1<\mathrm{CR}>$ IF $, \mathrm{I} 5,14 \mathrm{H}$ IS ACCEPTABLE )

READ (IN, 1070) NXTID

IF (NXTID .EQ. 0) GO TO 210

DO $200 \mathrm{NXT}=1$, NNODE

IF (NXTID .EQ. NODEID(NXT)) GO TO 210

200 CONTINUE

WRITE (IOUT, 1220)

GO TO 140

210 WRITE (IOUT, 1290) PLTX(INOD)

1290 FORMAT (51H ENTER THE X PLOTTING OFFSET (10 COLS) OR 〈CR> IF, 1 F $10.2,14 \mathrm{H}$ IS ACCEPTABLE )

READ (IN, 1040) PTX

IF (PTX .EQ. 0.) PTX = PLTX (INOD)

WRITE (IOUT, 1300) PLTY(INOD) 
Streamflow and Water-Quality Simulation--Continued

1300 FORMAT ( $51 \mathrm{H}$ ENTER THE Y PLOTTING OFFSET (10 COLS) OR <CR> IF ,

1 F $10.2,14 \mathrm{H}$ IS ACCEPTABLE )

READ (IN, 1040) PTY

IF (PTY .EQ. 0.) PTY = PLTY (INOD)

WRITE (IOUT, 1310) CONCFC (INOD)

1310 FORMAT (54H ENTER THE CONCENTRATION FACTOR (10 COLS) OR $\langle$ CR> IF ,

1 F $10.2,14 \mathrm{H}$ IS ACCEPTABLE )

READ (IN, 1040) CNCFAC

IF (CNCFAC .EQ. O.) CNCFAC = CONCFC (INOD)

WRITE (IOUT, 1320) DSA(INOD)

1320 FORMAT (5OH ENTER THE TDS/SC INTERCEPT (10 COLS) OR 〈CR> IF ,

1 F $10.2,14 \mathrm{H}$ IS ACCEPTABLE )

READ (IN, 1040) DDSA

IF (DDSA .EQ. 0.) DDSA = DSA (INOD)

WRITE (IOUT, 1330) DSB(INOD)

1330 FORMAT (46H ENTER THE TDS/SC SLOPE (10 COLS) OR <CR> IF ,F10.2,

$114 \mathrm{H}$ IS ACCEPTABLE )

READ (IN, 1040) DDSB

C

IF (DDSB .EQ. 0.) DDSB = DSB (INOD)

DO $220 \mathrm{~J}=1,12$

WRITE (IOUT, 1340) J, INDEPV(INOD, J)

1340 FORMAT (6HMONTH , I2,52H ENTER THE INDEPENDENT VARIABLE (5 COLS) 0 IR <CR> IF , I5, 14H IS ACCEPTABLE )

READ (IN, 1070) INDV(J)

$\operatorname{IF}(\operatorname{INDV}(\mathrm{J}) \cdot \mathrm{EQ} .0) \operatorname{INDV}(\mathrm{J})=\operatorname{INDEPV}(\mathrm{INOD}, \mathrm{J})$

WRITE (IOUT, 1350) NTRCPT (INOD, 'J)

1350 FORMAT (53H ENTER THE STREAMFLOW INTERCEPT (10 COLS) OR 〈CR> IF ,

1 F $10.4,14 \mathrm{H}$ IS ACCEPTABLE )

READ (IN, 1040) CEPT (J)

IF (CEPT (J) .EQ. O.) CEPT (J) $=\operatorname{NTRCPT}($ INOD, J $)$

WRITE (IOUT, 1360) SLOPE (INOD,J)

1360 FORMAT (5OH ENTER THE STREAMFLOW SLOPE (10 COLS) OR 〈CR> IF ,

1 F $10.4,14 \mathrm{H}$ IS ACCEPTABLE )

READ (IN, 1040) SLP(J)

$\operatorname{IF}(\operatorname{SLP}(J)$.EQ. 0.) $\operatorname{SLP}(J)=\operatorname{SLOPE}(\operatorname{INOD}, J)$

WRITE (IOUT, 1370) QWA(INOD,J)

1370 FORMAT (56H ENTER THE SC/STREAMFLOW INTERCEPT (10 COLS) OR 〈CR> I

$1 \mathrm{~F}, \mathrm{~F} 10.4,14 \mathrm{H}$ IS ACCEPTABLE )

READ (IN, 1040) QWAA(J)

IF (QWAA(J) .EQ. 0.) QWAA(J) = QWA $(I N O D, J)$

WRITE (IOUT, 1380) QWB (INOD,J)

1380 FORMAT (56H ENTER THE SC/STREAMFLOW SLOPE (10 COLS) OR 〈CR> IF ,

$1 \mathrm{~F} 10.4,14 \mathrm{H}$ IS ACCEPTABLE )

READ (IN , 1040) QWBB (J)

IF (QWBB (J) .EQ. 0.) QWBB $(J)=Q W B(I N O D, J)$

220 CONTINUE 
Attachment 7--FORTRAN SOURCE Code of Subroutines for

Streamflow and Water-Quality Simulation--Continued

1573

1574

1575

1576

1577

1578

1579

1580

1581

1582

1583

1584

1585

1586

1587

1588

1589

1590

1591

1592

1593

1594

1595

1596

1597

1598

1599

1600

1601

1602

1603

1604

1605

1606

1607

1608

1609

1610

1611

1612

1613

1614

1615

1616

1617

1618

1619

1620

1621

1622

1623

1624

1625

1626
C

DO $240 \mathrm{I}=1$, NNODE

INODE $=$ NNODE $-I+1$

IF (NXTNOD(INODE) .GT. INOD) NXTNOD(INODE) $=$ NXTNOD(INODE) +1

IF (INODE .LT. INOD) GO TO 240

IPLUS $=$ INODE +1

NODEID (IPLUS) = NODEID (INODE)

$\operatorname{NODNAM}($ IPLUS $)=$ NODNAM (INODE)

$X L O C($ IPLUS $)=X L O C$ (INODE)

YLOC (IPLUS) $=$ YLOC (INODE)

NXTNOD (IPLUS $)=$ NXTNOD (INODE)

$\operatorname{PLTX}($ IPLUS) $=$ PLTX (INODE)

PLTY (IPLUS) $=$ PLTY (INODE)

CONCFC (IPLUS) $=$ CONCFC (INODE)

DSA (IPLUS) $=$ DSA (INODE)

$\mathrm{DSB}($ IPLUS $)=\mathrm{DSB}$ (INODE)

DO $230 \mathrm{~J}=1,12$

NTRCPT (IPLUS,$J$ ) $=$ NTRCPT (INODE,$J$ )

SLOPE (IPLUS,$J$ ) = SLOPE (INODE,$J$ )

$\operatorname{INDEPV}($ IPLUS,$J)=\operatorname{INDEPV}($ INODE,$J)$

QWA(IPLUS,$J)=$ QWA (INODE,$J)$

$\mathrm{QWB}($ IPLUS,$J)=\mathrm{QWB}($ INODE, $\mathrm{J})$

230 CONTINUE

240 CONTINUE

C

IF (INOD .GT. NNODE) NXTNOD (NNODE) = INOD

NNODE $=$ NNODE +1

$\operatorname{NODEID}($ INOD $)=$ IADD

NODNAM (INOD) $=$ INAM

$X I O C$ (INOD) $=X L$

YLOC (INOD) $=Y L$

$\operatorname{NXTNOD(INOD)}=\mathrm{NXT}+1$

$\operatorname{PLTX}($ INOD) $=$ PTX

PLTY (INOD) $=$ PTY

CONCFC (INOD) = CNCFAC

$\mathrm{DSA}(\mathrm{INOD})=\mathrm{DDSA}$

$\mathrm{DSB}(\mathrm{INOD})=\mathrm{DDSB}$

C

D0 $250 \mathrm{~J}=1,12$

$\operatorname{NTRCPT}($ INOD,$J)=\operatorname{CEPT}(\mathrm{J})$

SLOPE $(I N O D, J)=\operatorname{SLP}(J)$

$\operatorname{INDEPV}(I N O D, J)=\operatorname{INDV}(J)$

QWA(INOD, J) $=$ QWAA(J)

$\mathrm{QWB}(\mathrm{INOD}, \mathrm{J})=\mathrm{QWBB}(\mathrm{J})$

250 CONTINUE

GO TO 120

C

$\mathrm{C}$

C

260 WRITE (IOUT, 1390)

1390 FORMAT (45H DO YOU WANT TO CHANGE THE REGRESSION VALUES? )

CALL QUESZN(IQ)

IF (IQ - 1) $290,270,290$

270 WRITE (IOUT, 1400) 
Attachment 7--FORTRAN SOURCE Code of Subroutines for Streamflow and Water-Quality Simulation--Continued

1627

1628

1629

1630

1631

1632

1633

1634

1635

1636

1637

1638

1639

1640

1641

1642

1643

1644

1645

1646

1647

1648

1649

1650

1651

1652

1653

1654

1655

1656

1657

1658

1659

1660

1661

1662

1663

1664

1665

1666

1667

1668

1669

1670

1671

1672

1673

1674

1675

1676

1677

1678

1679

1680
1400 FORMAT (31H ENTER THE NODE NUMBER (5 COLS))

READ (IN, 1030) INODE

DO $280 \mathrm{~J}=1,12$

WRITE (IOUT, 1340) J, INDEPV (INODE , J)

READ (IN, 1070) INDV(J)

IF (INDV $(J)$.NE. 0) INDEPV(INODE, J) $=\operatorname{INDV}(J)$

WRITE (IOUT, 1350) NTRCPT(INODE, J)

READ (IN, 1040) CEPT(J)

IF (CEPT $(\mathrm{J})$.NE . O.) NTRCPT $(\operatorname{INODE}, \mathrm{J})=\operatorname{CEPT}(\mathrm{J})$

WRITE (IOUT, 1360) SLOPE (INODE, J)

READ (IN, 1040) SLP(J)

IF (SLP(J) .NE. 0.) SLOPE (INODE, J) $=\operatorname{SLP}(\mathrm{J})$

WRITE (IOUT, 1370) QWA (INODE, J)

READ (IN, 1040) QWAA(J)

IF (QWAA(J) .NE. 0.) QWA(INODE, J) $=\operatorname{QWAA}(\mathrm{J})$

WRITE (IOUT , 1380) QWB (INODE, J)

READ (IN , 1040) QWBB (J)

280

IF (QWBB (J) .NE. 0.) QWB(INODE, J) $=$ QWBB $(J)$

C

C

C

290 WRITE (IOUT, 1410)

1410 FORMAT (68H DO YOU WANT TO CHANGE A CONCENTRATION FACTOR OR TDS/SC

1 COEFFICIENT? )

CALL QUESZN (IQ)

IF (IQ - 1) $310,300,310$

300 WRITE (IOUT, 1400)

READ (IN, 1030) INODE

WRITE (IOUT, 1310) CONCFC (INODE)

READ (IN, 1040) CNCFAC

IF (CNCFAC .NE. O.) CONCFC (INODE) = CNCFAC

WRITE (IOUT, 1320) DSA(INODE)

READ (IN, 1040) DDSA

IF (DDSA .NE. O.) DSA (INODE) = DDSA

WRITE (IOUT, 1330) DSB(INODE)

READ (IN, 1040) DDSB

IF (DDSB .NE. 0.) DSB (INODE) $=$ DDSB

GO TO 120

C

C

C

310 DO 320 INODE $=1$, NNODE

320 NODEUS (INODE) $=0$

DO 330 INODE $=1$, NNODE

NEXT $=$ NXTNOD (INODE)

IF (NODEUS (NEXT) .NE. 0) GO TO 330

NODEUS (NEXT) = INODE

330 CONTINUE

C

C

C

ENTER TITLE

WRITE (IOUT, 1420) 
Attachment 7--FORTRAN SOURCE Code of Subroutines for

Streamflow and Water-Quality Simulation--Continued

1681

1682

1683

1684

1685

1686

1687

1688

1689

1690

1691

1692

1693

1694

1695

1696

1697

1698

1699

1700

1701

1702

1703

1704

1705

1706

1707

1708

1709

1710

1711

1712

1713

1714

1715

1716

1717

1718

1719

1720

1721

1722

1723

1724

1725

1726

1727

1728

1729

1730

1731

1732

1420 FORMAT (//67H ENTER THE TITLE FOR THIS SIMULATION RUN (LIMITED TO 120 CHARACTERS ) )

READ (IN, 1000) TITL

JNUM $=28$

CALL TIMDAT (TODAY, JNUM)

$\mathrm{C}$

C WRITE BASIN DESCRIPTION DATA

$\mathrm{C}$

WRITE $(63,1430)$ TITL, (TODAY (JNUM), JNUM=1,3), TITLED, NNODE

1430 FORMAT (82H1 BSNMD2 - RIVER BASIN SIMULATION MODEL 非2 UPDATE

1D $9 / 10 / 86$ BY ALAN BURNS ,///,20H SIMULATION TITLE: ,5A4,

2 66X,10H DATE RUN: ,A2,1H/,A2,1H/,A2,///,

326H BASIN DESCRIPTION FILE: ,20A4,11H, CONTAINS , I3,6H NODES )

WRITE $(63,1440)$ TITLEP, NINDV, NMONTH

1440 FORMAT $(/ /, 29 \mathrm{H}$ INDEPENDENT VARIABLE FILE: $, 20 \mathrm{A4}, /, 10 \mathrm{X}, 8$ HCONTAINS $1,13,13 \mathrm{H}$ RECORDS FOR , I3,7H MONTHS )

WRITE $(63,1090)(I, \operatorname{NODEID}(I), \operatorname{NODNAM}(I), \operatorname{XLOC}(I), \operatorname{YLOC}(I), \operatorname{NXTNOD}(I)$,

$1 \operatorname{CONCFC}(I), \operatorname{DSA}(I), D S B(I), I=1, N N O D E)$

DO 390 NOD $=1$, NNODE

WRITE $(63,1110)$ NODEID (NOD), NODNAM(NOD)

DO $390 \mathrm{~J}=1,12$

$\mathrm{IND}=\operatorname{INDEPV}(\mathrm{NOD}, \mathrm{J})$

IF (IND .LT. 0) GO TO 360

IF (IND .GT. 100) GO TO 340

WRITE $(63,1120) \mathrm{J}, \operatorname{INDEPV}(\mathrm{NOD}, \mathrm{J}), \operatorname{VARNAM}(\mathrm{IND}), \operatorname{NTRCPT}(\mathrm{NOD}, \mathrm{J})$,

$1 \operatorname{SLOPE}(\mathrm{NOD}, \mathrm{J}), \mathrm{QWA}(\mathrm{NOD}, \mathrm{J}), \mathrm{QWB}(\mathrm{NOD}, \mathrm{J})$

GO TO 390

340 IND $=$ IND -100

IF (IND .GT. 100) GO TO 350

WRITE $(63,1130) \mathrm{J}, \operatorname{INDEPV}(\mathrm{NOD}, \mathrm{J}), \operatorname{VARNAM}(\mathrm{IND}), \operatorname{NTRCPT}(\mathrm{NOD}, \mathrm{J})$,

1 SLOPE (NOD, J), QWA(NOD, J), QWB (NOD , J)

GO TO 390

350 WRITE $(63,1140)$ J, INDEPV(NOD, J), NTRCPT(NOD , J) , SLOPE (NOD , J),

1 QWA(NOD, J), QWB (NOD, J)

GO TO 390

360 IND $=-$ IND

IF (IND .GT. 100) GO TO 370

WRITE $(63,1150) \mathrm{J}, \operatorname{INDEPV}(\mathrm{NOD}, \mathrm{J}), \operatorname{VARNAM}(\mathrm{IND}), \operatorname{NTRCPT}(\mathrm{NOD}, \mathrm{J})$,

1 SLOPE $(N O D, J), Q W A(N O D, J), Q W B(N O D, J)$

GO TO 390

$370 \mathrm{IND}=\mathrm{IND}-100$

IF (IND .GT. 100) GO TO 380

WRITE $(63,1160) \mathrm{J}$, INDEPV(NOD, J), VARNAM(IND) , NTRCPT (NOD , J) ,

1 SLOPE (NOD, J), QWA(NOD, J), QWB(NOD, J)

GO TO 390

380 WRITE $(63,1170) \mathrm{J}, \operatorname{INDEPV}(N O D, J), \operatorname{NTRCPT}(N O D, J), \operatorname{SLOPE}(N O D, J)$,

1 QWA (NOD, J), QWB(NOD, J)

C 390 CONTINUE

C

ENTER GRAPHICS TERMINAL INFORMATION

WRITE (IOUT, 1450) 
Attachment 7--FORTRAN SOURCE Code of Subroutines for

Streamflow and Water-Quality Simulation--Continued

1733

1734

1735

1736

1737

1738

1739

1740

1741

1742

1743

1744

1745

1746

1747

1748

1749

1750

1751

1752

1753

1754

1755

1756

1757

1758

1759

1760

1761

1762

1763

1764

1765

1766

1767

1768

1769

1770

1771

1772

1773

1774

1775

1776

1777

1778

1779

1780

1781

1782

1783

1784

1785

1786
1450 FORMAT (39H ENTER CODE FOR GRAPHICS DEVICE ( 1 COL) ,/,5X,

$111 \mathrm{HO}$ FOR NONE ,/,5X,15H1 FOR TEK $4010, /, 5 \mathrm{X}, 15 \mathrm{H} 2$ FOR TEK 4027 ,/,

2 5X,15H3 FOR TEK $4105, /, 5 \mathrm{X}, 15 \mathrm{H} 4$ FOR TEK 4107 ,/,

3 5X,15H5 FOR HP 7475 )

READ (IN , 1070) IGRAPH

IF (IGRAPH .EQ. 1) CALL TK4010(960)

IF (IGRAPH .EQ. 2) CALL TK4027

IF (IGRAPH .EQ. 3) CALL TK41(4105)

IF (IGRAPH .EQ. 4) CALL TK41(4107)

IF (IGRAPH .EQ. 5) CALL HP7475(1)

C

$\mathrm{C}$

C

\section{ENTER FREQUENCY OF PLOTTING SCHEMATIC}

WRITE (IOUT, 1460)

1460 FORMAT (6OH ENTER THE FREQUENCY OF PLOTTING THE FLOW SCHEMATIC (5 1COLS) $, /, 7 X, 18 \mathrm{HO}$ FOR NO SCHEMATIC ,/, 7X,13H1 FOR MONTHLY ,/, 7X, 2 15H12 FOR ANNUALLY ,/, 7X,34H99999 FOR PERIOD-OF-RECORD AVERAGE) READ (IN, 1070) ISKM

C

C ENTER NODES FOR HYDROGRAPHS

$\mathrm{C}$

WRITE (IOUT, 1470)

1470 FORMAT (65H ENTER THE NODE IDS FOR THOSE NODES AT WHICH YOU WANT H 1 YDROGRAPHS , /,7X,32H(5 COLS EACH - LIMIT OF 5 NODES) )

READ (IN, 1070) IHY

IF (ISKM .NE. 1) GO TO 420

DO $410 I=1,5$

$\mathrm{INODE}=\mathrm{IHY}(\mathrm{I})$

IF (INODE) $410,410,400$

400 WRITE (IOUT, 1480) INODE

1480 FORMAT (5OH ENTER THE APPROXIMATE MAXIMUM STREAMFLOW AT NODE, I5)

READ (IN, 1040) QHYMAX (I)

410 CONTINUE

420 DO $440 \mathrm{I}=1,5$

DO $430 \mathrm{~J}=1$, NNODE

IF (IHY(I) .NE. NODEID(J)) GO TO 430

$\mathrm{IHY}(\mathrm{I})=\mathrm{J}$

GO TO 440

430 CONTINUE

440 CONTINUE

C

C

C

\section{ENTER NODES FOR MONTHLY TABLES}

WRITE (IOUT, 1490)

1490 FORMAT (67H ENTER THE NODE IDS FOR THOSE NODES AT WHICH YOU WANT M 1ONTHLY FLOWS $, /, 7 \mathrm{X}, 32 \mathrm{H}$ ( 5 COLS EACH - LIMIT OF 5 NODES) )

READ (IN, 1070) ITBL

DO $460 \mathrm{I}=1,5$

DO $450 \mathrm{~J}=1$, NNODE

IF (ITBL(I) .NE. NODEID(J)) GO TO 450

$\operatorname{ITBL}(\mathrm{I})=\mathrm{J}$

GO TO 460

450 CONTINUE

460 CONTINUE 
Attachment 7--FORTRAN SOURCE Code of Subroutines for

Streamflow and Water-Quality Simulation--Continued

1787

1788

1789

1790

1791

1792

1793

1794

1795

1796

1797

1798

1799

1800

1801

1802

1803

1804

1805

1806

1807

1808

1809

1810

1811

1812

1813

1814

1815

1816

1817

1818

1819

1820

1821

1822

1823

1824

1825

1826

1827

1828

1829

1830

1831
C

C

C WRITE (IOUT, 1500)

1500 FORMAT (64H ENTER THE NODE IDS FOR THOSE NODES AT WHICH YOU WANT S 1TATISTICS $, /, 7 \mathrm{X}, 33 \mathrm{H}(5$ COLS EACH - LIMIT OF 10 NODES) )

READ (IN, 1070) ISS

DO $490 \mathrm{I}=1,10$

IF (ISS(I) .LE. 0) GO TO 490

DO $470 \mathrm{~J}=1,12$

$\operatorname{XNN}(I, J)=0$.

$\operatorname{QMEAN}(I, J)=0$.

$\operatorname{QSD}(I, J)=0$.

$\operatorname{QWMEAN}(I, J)=0$.

$\operatorname{QWSD}(I, J)=0$.

$\operatorname{QWLMN}(I, J)=0$.

$470 \operatorname{QWLSD}(\mathrm{I}, \mathrm{J})=0$.

DO $480 \mathrm{~J}=1$, NNODE

IF (ISS(I) .NE. NODEID(J)) GO TO 480

$\operatorname{ISS}(I)=J$

GO TO 490

480 CONTINUE

490 CONTINUE

C

C

C

IF (ISKM) $520,520,500$

500 WRITE (IOUT, 1510)

1510 FORMAT (49H DO YOU WANT TO PLOT THE NODE LOCATION SCHEMATIC? ) CALL QUESZN (IQ)

IF (IQ - 1) $520,510,520$

510 WRITE (IDUM, 1520) TITL

1520 FORMAT (5A4,18H - NODE LOCATIONS\$)

CALL PLTNOD(ITITLE)

C

520 WRITE (IOUT, 1530)

1530 FORMAT (54H THE MODEL NORMALLY COMPUTES A TIME-WEIGHTED QW VALUE.

$1, /, 54 \mathrm{H}$ DO YOU WANT TO COMPUTE A DISCHARGE-WEIGHTED QW VALUE? )

CALL QUESZN(IQW)

WRITE (IOUT, 1540)

1540 FORMAT (44H DO YOU WANT TO DISPLAY ALL MONTHLY VALUES? )

CALL QUESZN(IPRINT)

C

RETURN

END 
Attachment 7--FORTRAN SOURCE Code of Subroutines for Streamflow and Water-Quality Simulation--Continued

1832

1833

1834

1835

1836

1837

1838

1839

1840

1841

1842

1843

1844

1845

1846

1847

1848

1849

1850

1851

1852

1853

1854

1855

1856

1857

1858

1859

1860

1861

1862

1863

1864

1865

1866

1867

1868

1869

1870

1871

1872

1873

1874

1875

1876

1877

1878

1879

SUBROUTINE RKRLAB (MONTH, YMAX, YMIN)

C

YINC $=($ YMAX - YMIN $) / 50$

YPLOT $=-$ YMAX $/ 15$.

IRECUR $=99$

$X=100$ - IRECUR

$X=$ FUNCZ $(X / 100$.

CALL RLINT (IRECUR, $X, Y P L O T$ )

CALL RLVEC (X, YMIN, $X, Y M I N+Y I N C, 0)$

CALL RLVEC (X, YMAX, $X$, YMAX-YINC, 0$)$

IRECUR $=97$

$X=100$ - IRECUR

$X=\operatorname{FUNCZ}(X / 100$.

CALL RLINT (IRECUR, $X, Y P L O T$ )

CALL RLVEC (X, YMIN, $X, Y M I N+Y I N C, 0)$

CALL RLVEC $(X, Y M A X, X$, YMAX-YINC, 0$)$

IRECUR $=90$

$X=100$ - IRECUR

$X=F U N C Z(X / 100$.

CALL RLINT (IRECUR, $X, Y P L O T$ )

CALL RLVEC (X, YMIN, $X, Y M I N+Y I N C, 0)$

CALL RLVEC (X, YMAX, $X$, YMAX-YINC , 0$)$

IRECUR $=50$

$X=100$ - IRECUR

$X=F U N C Z(X / 100$.

CALL RLINT (IRECUR, $X, Y P L O T$ )

CALL RLVEC (X, YMIN , X, YMIN+YINC , 0)

CALL RLVEC (X, YMAX, X, YMAX-YINC , 0)

IRECUR $=10$

$X=100$ - IRECUR

$X=\operatorname{FUNCZ}(X / 100$.

CALL RLINT (IRECUR, $X, Y P L O T$ )

CALL RLVEC (X, YMIN, $X, Y M I N+Y I N C, 0)$

CALL RLVEC (X, YMAX, $X$, YMAX-YINC, 0$)$

IRECUR $=3$

$X=100$ - IRECUR

$X=\operatorname{FUNCZ}(X / 100$.

CALL RLINT (IRECUR, $X, Y P L O T$ )

CALL RLVEC (X, YMIN, X, YMIN+YINC, 0)

CALL RLVEC $(X$, YMAX, $X, Y M A X-Y I N C, 0)$

IRECUR $=1$

$X=100$ - IRECUR

$X=F U N C Z(X / 100$.

CALL RLINT (IRECUR, $X, Y P L O T$ )

CALL RLVEC (X, YMIN, $X, Y M I N+Y I N C, 0)$

CALL RLVEC (X, YMAX, $X$, YMAX-YINC , 0$)$

RETURN

END 
Attachment 7--FORTRAN SOURCE Code of Subroutines for Streamflow and Water-Quality Simulation--Continued

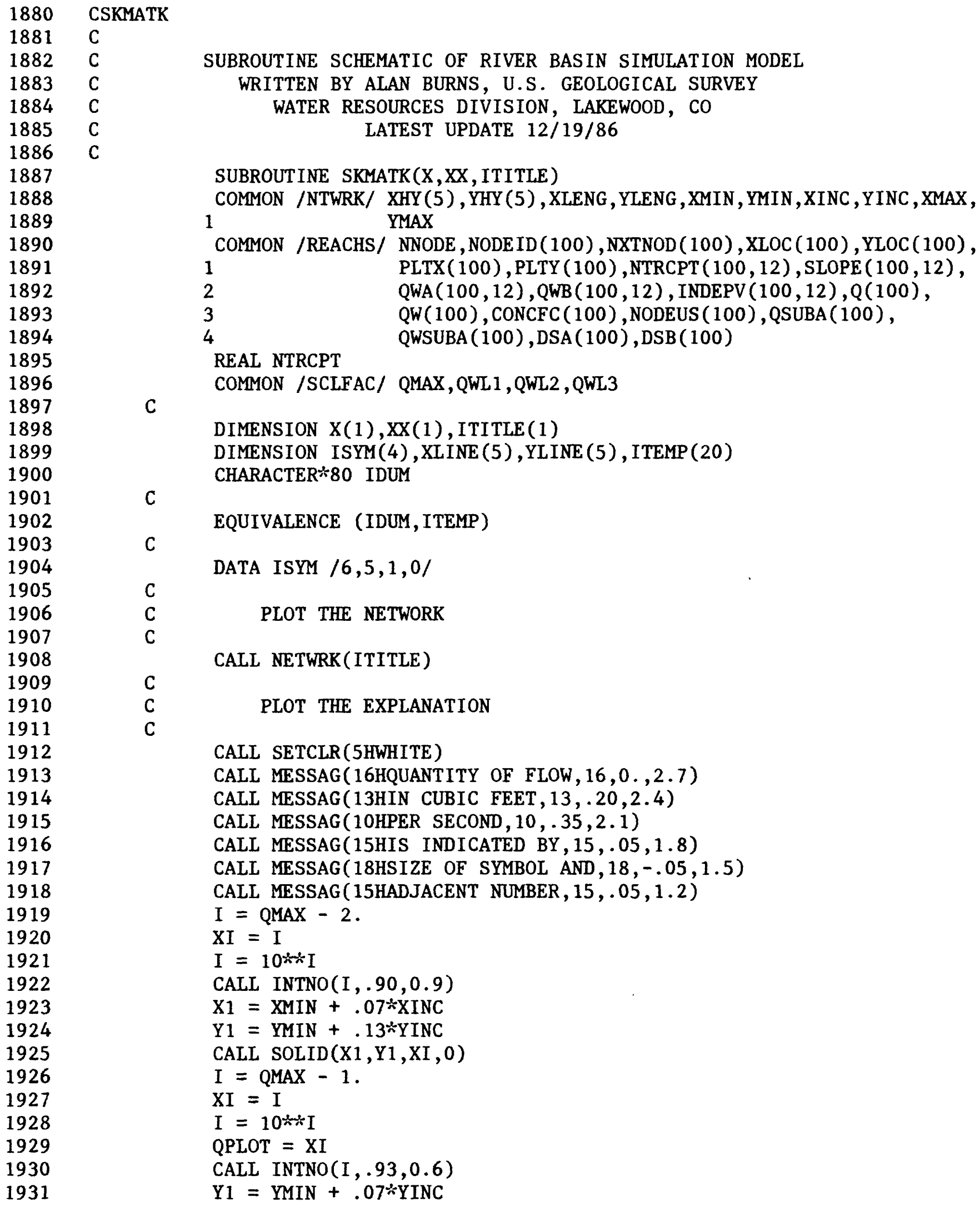


Attachment 7--FORTRAN SOURCE Code of Subroutines for Streamflow and Water-Quality Simulation--Continued

1932

1933

1934

1935

1936

1937

1938

1939

1940

1941

1942

1943

1944

1945

1946

1947

1948

1949

1950

1951

1952

1953

1954

1955

1956

1957

1958

1959

1960

1961

1962

1963

1964

1965

1966

1967

1968

1969

1970

1971

1972

1973

1974

1975

CALL SOLID $(\mathrm{X} 1, \mathrm{Y} 1, \mathrm{XI}, 0)$

$\mathrm{I}=\mathrm{QMAX}$

$\mathrm{XI}=\mathrm{I}$

$I=10 \cdots \mathrm{I}$

CALL INTNO(I, $.95,0.25)$

$\mathrm{Y} 1=$ YMIN

CALL SOLID $(\mathrm{X} 1, \mathrm{Y} 1, \mathrm{XI}, 0)$

CALL MESSAG(15HQUALITY OF FLOW, 15,8.,2.1)

CALL MESSAG(23HIN MILLIGRAMS PER LITER, 23,7.45, 1.8)

CALL MESSAG(21HIS INDICATED BY SHAPE, 21, 7.65, 1.5)

CALL MESSAG(19HAND COLOR OF SYMBOL, 19,7.8,1.2)

CALL SETCLR (4HCYAN)

$\mathrm{X} 1=\mathrm{XMAX}-.17 * \mathrm{XINC}$

$\mathrm{Y} 1=\mathrm{YMIN}+.15 \div \mathrm{YINC}$

CALL SOLID (X1, Y1, QPLOT, ISYM(1))

WRITE (IDUM, 1000) QWL1

1000 FORMAT (1H<,F5.0)

CALL MESSAG(ITEMP , 6, 8.5,0.9)

CALL SETCLR (7HMAGENTA)

$\mathrm{Y} 1=$ YMIN $+.10 *$ YINC

CALL SOLID (X1, Y1, QPLOT, ISYM(2))

WRITE (IDUM, 1010) QWL1, QWL2

1010 FORMAT (F5.0,3H - ,F5.0)

CALL MESSAG(ITEMP, 13,8.5,0.6)

CALL SETCLR (6HYELLOW)

$\mathrm{Y} 1=\mathrm{YMIN}+.06 * \mathrm{YINC}$

CALL SOLID (X1, Y1 ,QPLOT, ISYM(3))

WRITE (IDUM, 1010) QWL2, QWL3

CALL MESSAG(ITEMP , 13,8 .5, 0.3)

CALL SETCLR(3HRED)

$\mathrm{Y} 1=$ YMIN $+.01 *$ YINC

CALL SOLID (X1, Y1,QPLOT, ISYM (4))

WRITE (IDUM, 1020) QWL3

1020 FORMAT (1H>,F5.0)

CALL MESSAG(ITEMP, $6,8.5,0$.

C

C

C

PLOT THE DATA

CALL SETCLR(5HWHITE)

DO 10 INODE $=1$, NNODE

$\mathrm{X} 1=\mathrm{XPOSN}(\mathrm{XLOC}(\mathrm{INODE}), \mathrm{YLOC}(\mathrm{INODE}))+\operatorname{PLTX}(\mathrm{INODE})$

Y1 $=$ YPOSN $(X L O C(I N O D E), Y L O C(I N O D E))+\operatorname{PLTY}($ INODE $)$

$\mathrm{JQ}=\mathrm{X}(\mathrm{INODE})+.5$

10 CALL INTNO(JQ, X1, Y1) 
Attachment 7--FORTRAN SOURCE Code of Subroutines for Streamflow and Water-Quality Simulation--Continued

1976

1977

1978

1979

1980

1981

1982

1983

1984

1985

1986

1987

1988

1989

1990

1991

1992

1993

1994

1995

1996

1997
C

DO 30 INODE $=1$, NNODE

IPEN $=1$

CALL SETCLR(4HCYAN)

IF (XX(INODE) .LT. QWL1) GO TO 20

IPEN $=2$

CALL SETCLR(7HMAGENTA)

IF (XX (INODE) .LT. QWL2) GO TO 20

IPEN $=3$

CALL SETCLR (6HYELLOW)

IF (XX(INODE) .LT. QWL3) GO TO 20

IPEN $=4$

CALL SETCLR(3HRED)

20 CONTINUE

IF (X(INODE) .LE. O.) GO TO 30

$\mathrm{FAC}=\operatorname{ALOG10}(\mathrm{X}(\mathrm{INODE}))$

CALL SOLID (XLOC (INODE), YLOC (INODE), FAC, ISYM(IPEN))

30 CONTINUE

CALL ENDPL(0)

C

RETURN

END 
Attachment 7--FORTRAN SOURCE Code of Subroutines for Streamflow and Water-Quality Simulation--Continued

1998 CSOLID

$1999 \mathrm{C}$

2000 C

2001 C

2002 C

2003 C

2004 C

2005

2006

2007

2008

2009

2010

2011

2012

2013

2014

2015

2016

2017

2018

2019

C

C
SUBROUTINE SOLID OF RIVER BASIN SIMULATION MODEL WRITTEN BY ALAN BURNS, U.S. GEOLOGICAL SURVEY WATER RESOURCES DIVISION, LAKEWOOD, CO LATEST UPDATE $9 / 10 / 86$

SUBROUTINE SOLID (X, Y, FAC, IMARK) COMMON /SCLFAC/ QMAX,QWL1,QWL2,QWL3

IFAC $=$ FAC $* 12 /$ QMAX

$\mathrm{IFAC}=\mathrm{IFAC}+1$

DO 10 II $=1$, IFAC

$\mathrm{FAC1}=\mathrm{II}$

$\mathrm{FAC} 1=\mathrm{FAC} 1 / 3$.

CALL MARKER (IMARK)

CALL SCLPIC (FAC1)

CALL HWSHD

10 CALL CURVE $(\mathrm{X}, \mathrm{Y}, 1,-1)$

RETURN

END 
Attachment 7--FORTRAN SOURCE Code of Subroutines for Streamflow and Water-Quality Simulation--Continued

2020

2021

2022

2023

2024

2025

2026

2027

2028

2029

2030

2031

2032

2033

2034

2035

2036

2037

2038

2039

2040

2041

2042
C

C

SUBROUTINE SORT (ARRAY,NPTS)

DIMENSION ARRAY(1)

DO $10 \mathrm{~K}=1,100$

IF (2* 2 K . GT. NPTS) GO TO 20

10 CONTINUE

$20 \mathrm{~K}=\mathrm{K}-1$

30 IF (K .EQ. 0) RETURN

$I=1$

$40 \mathrm{~J}=\mathrm{I}$

$\mathrm{Y}=\operatorname{ARRAY}(\mathrm{I}+\mathrm{K})$

50 IF (Y - ARRAY (J)) $80,60,60$

$60 \operatorname{ARRAY}(\mathrm{J}+\mathrm{K})=\mathrm{Y}$

$I=I+1$

IF (I+K-NPTS) $40,40,70$

70 IF (K .EQ. 2) GO TO 20

$\mathrm{K}=(\mathrm{K}-1) / 2$

GO TO 30

$80 \operatorname{ARRAY}(\mathrm{J}+\mathrm{K})=\operatorname{ARRAY}(\mathrm{J})$

$J=J-K$

IF (J) $60,60,50$

END 
Attachment 7--FORTRAN SOURCE Code of Subroutines for

Streamflow and Water-Quality Simulation--Continued

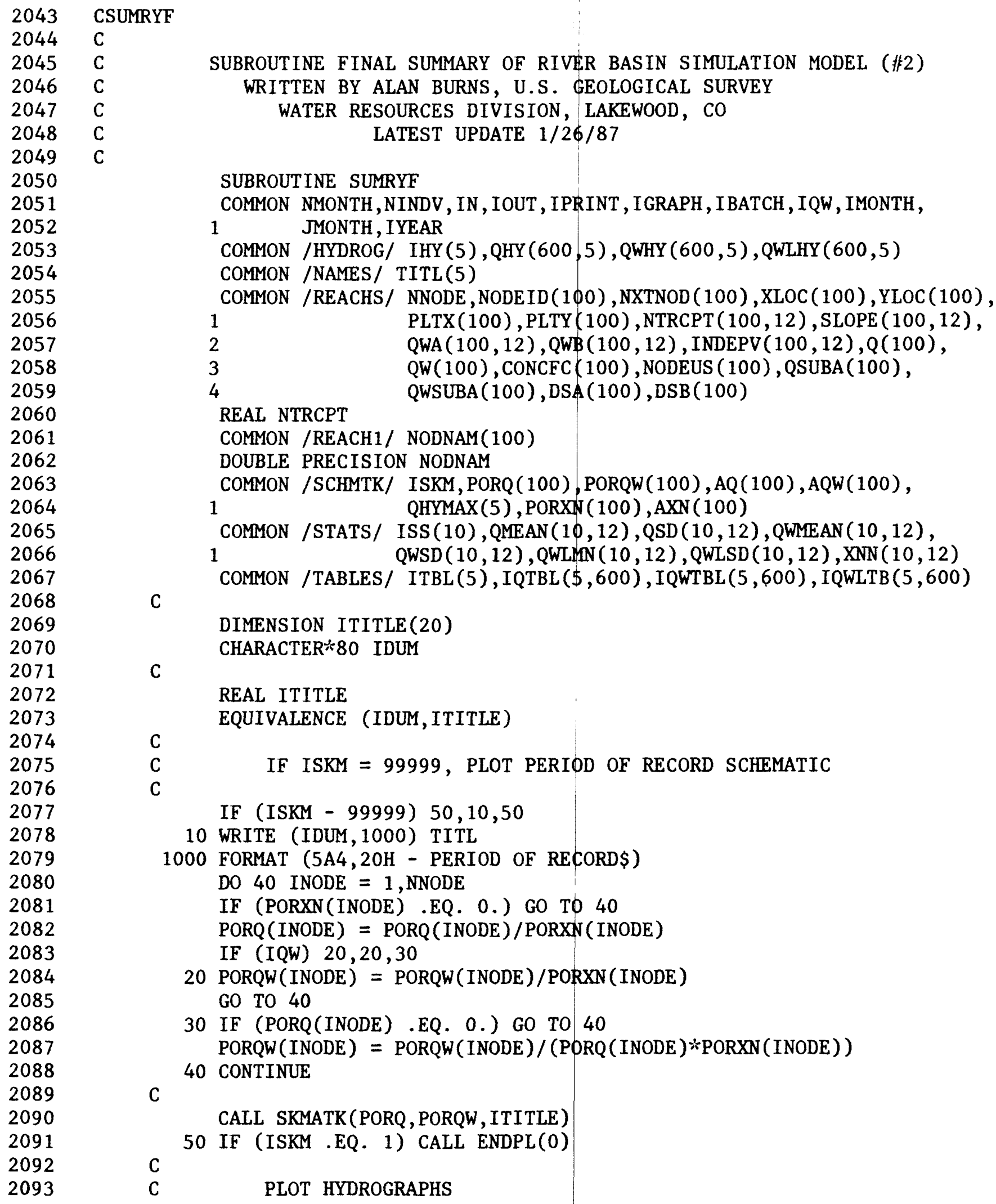


Attachment 7--FORTRAN SOURCE Code of Subroutines for

Streamflow and Water-Quality Simulation--Continued

2094

2095

2096

2097

2098

2099

2100

2101

2102

2103

2104

2105

2106

2107

2108

2109

2110

2111

2112

2113

2114

2115

2116

2117

2118

2119

2120

2121

2122

2123

2124

2125

2126

2127

2128

2129

2130

2131

2132

2133

2134

2135

2136

2137

2138

2139

2140

2141

2142

2143

2144

2145

2146

2147

C

DO $70 \quad I=1,5$

INODE $=$ IHY $(I)$

IF (INODE) $60,70,60$

60 WRITE (IDUM, 1010) TITL, NODEID(INODE), NODNAM(INODE)

1010 FORMAT $(5 \mathrm{~A} 4,3 \mathrm{H}-, \mathrm{I} 5,2 \mathrm{H}(, \mathrm{A} 8,2 \mathrm{H}) \$)$

CALL HYDRGR $(\mathrm{QHY}(1, \mathrm{I})$, ITITLE, 1$)$

CALL HYDRGR (QWHY $(1, I)$, ITITLE , 2)

CALL HYDRGR $(Q W L H Y(1, I), \operatorname{ITITLE}, 3)$

70 CONTINUE

NYEAR $=$ NMONTH $/ 12$

C

C LIST TABLES

C

DO $170 I=1,5$

INODE $=\operatorname{ITBL}(\mathrm{I})$

IF (INODE) $80,170,80$

C

80 DO 160 ITB $=1,3$

IF (IBATCH .EQ. 0) GO TO 90

WRITE (IOUT, 1020)

1020 FORMAT (////,37H IS YOUR TERMINAL READY FOR THE TABLE)

CALL QUESZN(IQ)

IF (IQ - 1) $160,90,160$

90 IF (ITB - 2) $100,120,140$

100 WRITE (IOUT, 1030) NODEID (INODE), NODNAM (INODE), $(\mathrm{J}, \mathrm{J}=1,12$ )

1030 FORMAT $(/ / / /, 50 \mathrm{H}$ MONTHLY FLOWS, IN CUBIC FEET PER SECOND, AT NODE $1, \mathrm{I} 5,2 \mathrm{H}(, \mathrm{A} 8,1 \mathrm{H}), / /, 6 \mathrm{H}$ MONTH, I3, 11I6,/,5H YEAR)

C

DO 110 IYEAR $=1$, NYEAR

JMONTH $=($ IYEAR -1$) * 12+1$

KMONTH $=$ IYEAR $\div 12$

110 WRITE (IOUT, 1040) IYEAR, (IQTBL ( I , IMONTH), IMONTH=JMONTH, KMONTH)

1040 FORMAT $(14,17,1116)$

GO TO 160

120 WRITE (IOUT, 1050) NODEID (INODE), NODNAM (INODE), ( $\mathrm{J}, \mathrm{J}=1,12$ )

1050 FORMAT (////,58H MONTHLY CONCENTRATIONS, IN MILLIGRAMS PER LITER,

C

1AT NODE , I5,2H $(, \mathrm{A} 8,1 \mathrm{H}), / /, 6 \mathrm{H}$ MONTH, I3,11I6,/,5H YEAR)

DO 130 IYEAR $=1$, NYEAR

JMONTH $=($ IYEAR -1$) * 12+1$

KMONTH $=$ IYEAR $* 12$

130 WRITE (IOUT, 1040) IYEAR, (IQWTBL ( I , IMONTH), IMONTH=JMONTH, KMONTH) GO TO 160

140 WRITE (IOUT, 1060) NODEID(INODE), NODNAM(INODE), $(\mathrm{J}, \mathrm{J}=1,12$ )

1060 FORMAT (////,33H MONTHLY LOADS, IN TONS, AT NODE , I5,2H (,A8, $1 \mathrm{1H}), / /, 6 \mathrm{H}$ MONTH, I3,1116,/,5H YEAR)

C

DO 150 IYEAR $=1$, NYEAR

JMONTH $=($ IYEAR -1$) \div 12+1$

$\mathrm{KMONTH}=\mathrm{IYEAR} * 12$

150 WRITE (IOUT, 1040) IYEAR, (IQWLTB (I , IMONTH), IMONTH=JMONTH, KMONTH)

160 CONTINUE

170 CONTINUE 
Attachment 7--FORTRAN SOURCE Code of Subroutines for

Streamflow and Water-Quality Simulation--Continued

2148

2149

2150

2151

2152

2153

2154

2155

2156

2157

2158

2159

2160

2161

2162

2163

2164

2165

2166

2167

2168

2169

2170

2171

2172

2173

2174

2175

2176

2177

2178

2179

2180

2181

2182

2183

2184

2185

2186

2187

2188

2189

2190

2191

2192

2193

2194

2195

2196

2197

2198

2199

2200
C

C LIST STATISTICS

$\mathrm{C}$

DO $210 \mathrm{I}=1,10$

INODE $=\operatorname{ISS}(\mathrm{I})$

IF (INODE) $180,210,180$

180 IF (IBATCH .EQ. 0) GO TO 190 WRITE (IOUT, 1070)

1070 FORMAT $(/ / / /, 42 \mathrm{H}$ IS YOUR TERMINAL READY FOR THE STATISTICS ) CALL QUESZN (IQ)

IF (IQ - 1) $210,190,210$

190 WRITE (IOUT, 1080) NODEID (INODE), NODNAM (INODE)

1080 FORMAT $(/ / / /, 32 \mathrm{H}$ THE MONTHLY STATISTICS AT NODE, I5, $2 \mathrm{H}(, \mathrm{A} 8,7 \mathrm{H})$ WE $1 \mathrm{RE}:, / /, 54 \mathrm{H}$ MONTH

$2 /, 15 \mathrm{X}, 40 \mathrm{H}$ (CUBIC

3 14X, 27HFEET PER

$410 \mathrm{X}, 3(17 \mathrm{H}$ MEAN STD DEV ))

$\mathrm{TQM}=0$.

TQSD $=0$.

$\mathrm{TQWM}=0$.

TQWSD $=0$.

TQWLM $=0$.

TQWLSD $=0$.

DO 200 JMONTH $=1,12$

$\mathrm{XM1}=-99$.

$\mathrm{XSD} 1=-99$.

$\mathrm{XM} 2=-99$.

$\mathrm{XSD} 2=-99$.

$\mathrm{XM} 3=-99$.

$\mathrm{XSD} 3=-99$.

$\mathrm{XNYEAR}=\mathrm{XNN}(\mathrm{I}, \mathrm{JMONTH})$

IF (XNYEAR .LE. 1.) GO TO 200

$\mathrm{TQM}=\mathrm{TQM}+$ QMEAN (I, JMONTH)

$\mathrm{TQSD}=\mathrm{TQSD}+\mathrm{QSD}(\mathrm{I}, \mathrm{JMONTH})$

TQWM $=$ TQWM + QWMEAN $(I$, JMONTH $)$

TQWSD $=$ TQWSD + QWSD $(I$, JMONTH $)$

TQWLM $=$ TQWLM + QWLMN $(I$, JMONTH $)$

TQWLSD $=$ TQWLSD + QWLSD ( $I$, JMONTH $)$

$\mathrm{XXN}=\mathrm{XNYEAR}-1$.

$\mathrm{XM1}=$ QMEAN $(\mathrm{I}, \mathrm{JMONTH}) / \mathrm{XNYEAR}$

$\mathrm{XSD} 1=(\mathrm{QSD}(\mathrm{I}, \mathrm{JMONTH})-\mathrm{QMEAN}(\mathrm{I}, \mathrm{JMONTH}) \div \mathrm{XM1}) / \mathrm{XXN}$

IF (XSD1 .LT. 0.) XSD1 $=0$.

$\mathrm{XSD} 1=\mathrm{SQRT}(\mathrm{XSD} 1)$

$\mathrm{XM} 2=$ QWMEAN $(I$, JMONTH $) / X N Y E A R$

$\mathrm{XSD} 2=(\mathrm{QWSD}(\mathrm{I}, \mathrm{JMONTH})-\mathrm{QWMEAN}(\mathrm{I}, \mathrm{JMONTH}) * \mathrm{XM} 2) / \mathrm{XXN}$

IF (XSD2 .LT . 0.) XSD2 $=0$.

$\mathrm{XSD} 2=\mathrm{SQRT}(\mathrm{XSD} 2)$

IF (IQW .EQ. 1) XM2 $=Q W L M N(I, J M O N T H) /(.082188 * Q M E A N(I, J M O N T H))$

$\mathrm{XM} 3=\mathrm{QWLMN}(\mathrm{I}, \mathrm{JMONTH}) / \mathrm{XNYEAR}$

$\mathrm{XSD} 3=(\mathrm{QWLSD}(\mathrm{I}, \mathrm{JMONTH})-\mathrm{QWLMN}(\mathrm{I}, \mathrm{JMONTH}) * \mathrm{XM} 3) / \mathrm{XXN}$

IF (XSD3 .LT. 0.) XSD3 $=0$.

XSD3 $=$ SQRT $(X S D 3)$

200 WRITE (IOUT, 1090) JMONTH, XM1 , XSD1 , XM2 , XSD2 , XM3 , XSD3 
Attachment 7--FORTRAN SOURCE Code of Subroutines for Streamflow and Water-Quality Simulation--Continued

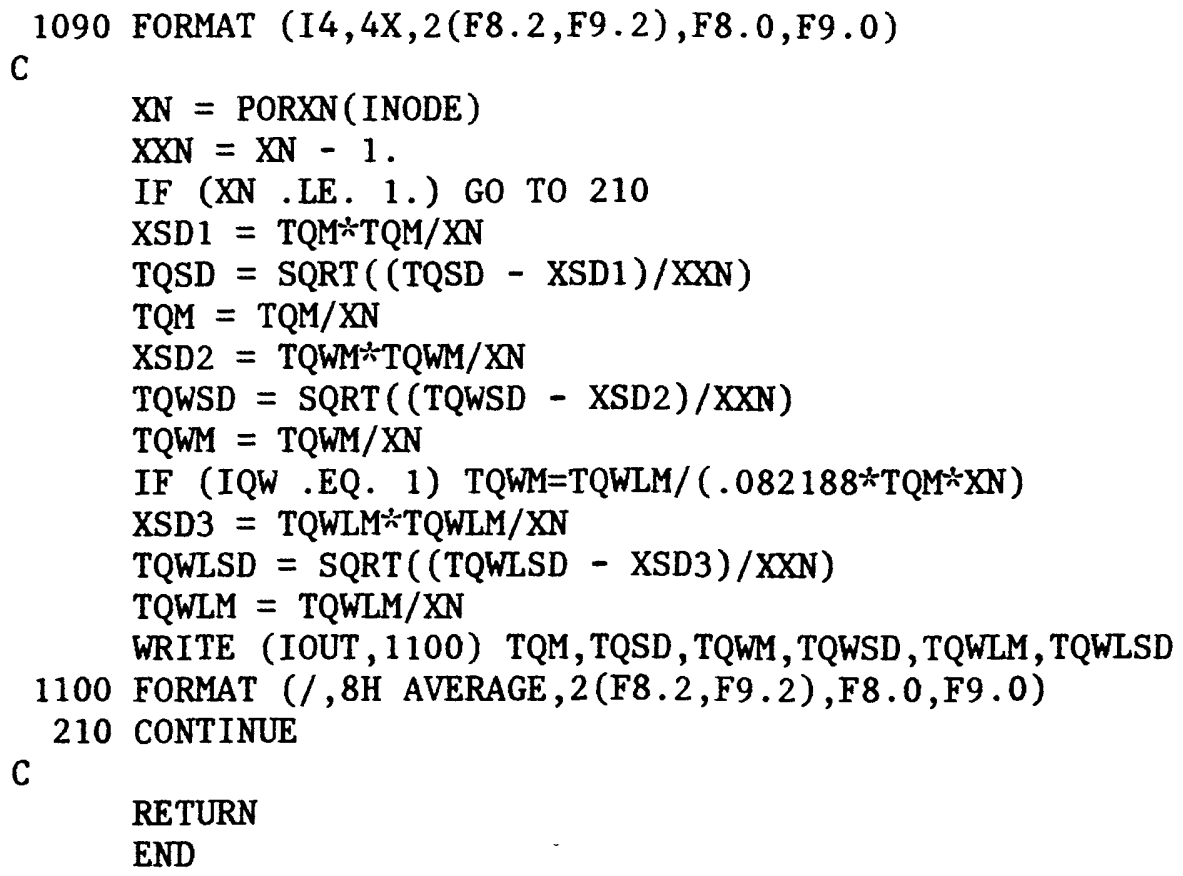


Attachment 7--FORTRAN SOURCE Code of Subroutines for

Streamflow and Water-Quality Simulation--Continued

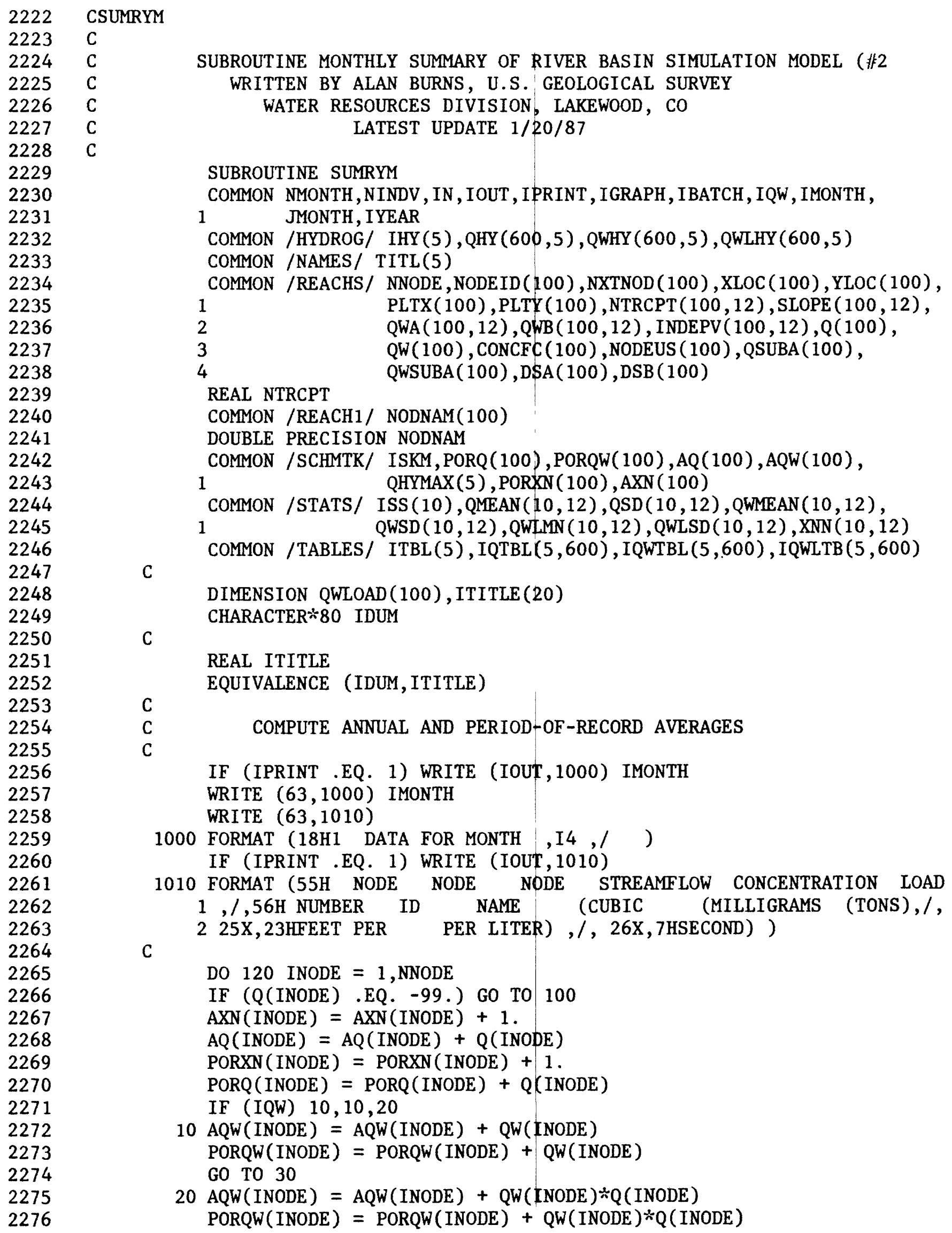

SUBROUTINE MONTHLY SUMMARY OF RIVER BASIN SIMULATION MODEL (非2 WRITTEN BY ALAN BURNS, U.S. GEOLOGICAL SURVEY WATER RESOURCES DIVISION, LAKEWOOD, CO LATEST UPDATE $1 / 20 / 87$

SUBROUTINE SUMRYM

COMMON NMONTH, NINDV , IN , IOUT, IPRINT , IGRAPH, IBATCH, IQW , IMONTH, 1 JMONTH, IYEAR

COMMON /HYDROG/ IHY (5), QHY $(600,5), Q W H Y(600,5), Q W L H Y(600,5)$

COMMON /NAMES/ TITL(5)

COMMON /REACHS/ NNODE, NODEID (100), NXTNOD (100), XLOC (100), YLOC (100), 1

2

3

4

REAL NTRCPT PLTX (100), PLTY (100), NTRCPT $(100,12), \operatorname{SLOPE}(100,12)$, QWA $(100,12), Q W B(100,12), \operatorname{INDEPV}(100,12), Q(100)$, QW(100), CONCFC (100), NODEUS (100), QSUBA (100), COMMON /REACH1/ NODNAM(100)

DOUBLE PRECISION NODNAM COMMON /SCHMTK/ ISKM,PORQ(100), PORQW(100), AQ(100), AQW(100), 1 QHYMAX (5), PORXN (100), AXN (100)

COMMON /STATS/ ISS $(10), \operatorname{QMEAN}(10,12), \operatorname{QSD}(10,12), \operatorname{QWMEAN}(10,12)$, $1 \quad \operatorname{QWSD}(10,12), \operatorname{QWLMN}(10,12), \operatorname{QWLSD}(10,12), \operatorname{XNN}(10,12)$ COMMON /TABLES/ ITBL $(5), \operatorname{IQTBL}(5,600), \operatorname{IQWTBL}(5,600), \operatorname{IQWLTB}(5,600)$

C

$\mathrm{C}$

DIMENSION QWLOAD (100), ITITLE (20)

CHARACTER $\div 80$ IDUM

REAL ITITLE

EQUIVALENCE (IDUM, ITITLE)

C

C

COMPUTE ANNUAL AND PERIOD-OF-RECORD AVERAGES

$\mathrm{C}$

IF (IPRINT .EQ. 1) WRITE (IOUT, 1000) IMONTH

WRITE $(63,1000)$ IMONTH

WRITE $(63,1010)$

1000 FORMAT (18H1 DATA FOR MONTH , I4,/ )

IF (IPRINT .EQ. 1) WRITE (IOUT, 1010)

1010 FORMAT (55H NODE NODE NODE STREAMFLOW CONCENTRATION LOAD $1, /, 56 \mathrm{H}$ NUMBER ID NAME (CUBIC (MILLIGRAMS (TONS),/,

2 25X, 23HFEET PER PER LITER) ,/, 26X,7HSECOND) )

C

DO 120 INODE $=1$, NNODE

IF ( $Q$ (INODE) .EQ. -99.) GO TO 100

$\operatorname{AXN}($ INODE $)=A X N(I N O D E)+1$.

$A Q($ INODE $)=A Q($ INODE $)+Q($ INODE $)$

PORXN (INODE) $=$ PORXN (INODE) +1 .

PORQ (INODE) $=$ PORQ $($ INODE $)+Q($ INODE $)$

IF (IQW) $10,10,20$

$10 \mathrm{AQW}($ INODE $)=\mathrm{AQW}($ INODE $)+\mathrm{QW}($ INODE $)$

PORQW $($ INODE $)=$ PORQW $($ INODE $)+Q W($ INODE $)$

GO TO 30

$20 \mathrm{AQW}($ INODE $)=\mathrm{AQW}($ INODE $)+\mathrm{QW}($ INODE $) \div Q($ INODE $)$

$P O R Q W($ INODE $)=P O R Q W($ INODE $)+Q W($ INODE $) * Q($ INODE $)$ 
Attachment 7--FORTRAN SOURCE Code of Subroutines for Streamflow and Water-Quality Simulation--Continued

30 QWLOAD (INODE) $=Q($ INODE $) * Q W($ INODE $) * .0027 * 30.44$

IF ( $Q$ (INODE) .LT. O.) $Q(I N O D E)=0$.

IF (QW(INODE) .LT. O.) $\mathrm{QW}($ INODE) $=0$.

IF (QWLOAD(INODE) .LT. 0.) QWLOAD (INODE) $=0$.

IF (QW(INODE) .GT. 100000.) QW(INODE) $=-99$.

IF (QWLOAD(INODE) .GT. 10000000.) QWLOAD(INODE) $=-99$.

C

C

C

STORE HYDROGRAPH DATA

DO $50 \mathrm{I}=1,5$

IF (INODE - IHY(I)) $50,40,50$

$40 \mathrm{QHY}(\mathrm{IMONTH}, \mathrm{I})=\mathrm{Q}$ (INODE)

QWHY $($ IMONTH, I $)=$ QW (INODE)

QWLHY (IMONTH, I) = QWLOAD (INODE)

50 CONTINUE

C

C

C

DO $70 \mathrm{I}=1,5$

IF (INODE - ITBL(I)) $70,60,70$

$60 \mathrm{IQTBL}(\mathrm{I}, \mathrm{IMONTH})=\mathrm{Q}(\mathrm{INODE})+.5$

$\operatorname{IQWTBL}(\mathrm{I}, \mathrm{IMONTH})=\mathrm{QW}(\mathrm{INODE})+.5$

70 CONTINUE

$\operatorname{IQWLTB}(I$, IMONTH $)=$ QWLOAD $($ INODE $)+.5$

C

$\mathrm{C}$

C

\section{STORE STATISTICS DATA}

DO $90 \mathrm{I}=1,10$

IF (INODE - ISS(I)) $90,80,90$

$80 \operatorname{XNN}(I$, JMONTH $)=\operatorname{XNN}(I$, JMONTH $)+1$.

QMEAN $(I, J M O N T H)=\operatorname{QMEAN}(I, J M O N T H)+Q$ (INODE)

$\mathrm{QSD}(\mathrm{I}, \mathrm{JMONTH})=\mathrm{QSD}(\mathrm{I}, \mathrm{JMONTH})+\mathrm{Q}(\mathrm{INODE}) * \mathrm{Q}$ (INODE)

QWMEAN (I , JMONTH) = QWMEAN (I, JMONTH) + QW (INODE)

QWSD $(I, J M O N T H)=$ QWSD $(I, J M O N T H)+Q W(I N O D E) \div Q W($ INODE $)$

QWLMN $(I, J M O N T H)=$ QWLMN $(I, J M O N T H)+Q W L O A D$ (INODE)

QWLSD $(I, J M O N T H)=$ QWLSD $(I$, JMONTH $)+$ QWLOAD (INODE) $*$ QWLOAD (INODE)

90 CONTINUE

GO TO 110

C

$100 \mathrm{QWLOAD}($ INODE $)=\mathrm{Q}($ INODE $)$

$\mathrm{QW}(\mathrm{INODE})=\mathrm{Q}(\mathrm{INODE})$

110 IF (IPRINT .EQ. 1) WRITE (IOUT, 1020) INODE, NODEID(INODE),

1 NODNAM(INODE), Q(INODE), QW(INODE), QWLOAD (INODE)

WRITE $(63,1020)$ INODE, NODE ID (INODE), NODNAM(INODE), Q(INODE),

1 QW (INODE), QWLOAD (INODE)

1020 FORMAT (I6,I7, 2X,A8,F11.2,F13.0,F11.2)

120 CONTINUE 
Attachment 7--FORTRAN SOURCE Code of Subroutines for Streamflow and Water-Quality Simulation--Continued

C

C IF ISKM $=1$, PLOT MONTHLY SCHEMATIC

C

IF (ISKM - 1) $210,130,140$

130 CALL MONTHA

GO TO 210

C

140 IF (ISKM - 12) $210,150,210$

C

$\mathrm{C}$

150 IF (JMONTH - 12) $210,160,210$

160 WRITE (IDUM, 1030) TITL, IYEAR

1030 FORMAT $(5 A 4,8 \mathrm{H}$ - YEAR , I3, 1H\$)

DO 190 INODE $=1$, NNODE

IF (AXN(INODE) .EQ. O.) GO TO 190

$A Q(I N O D E)=A Q(I N O D E) / A X N$ (INODE)

IF (IQW) $170,170,180$

$170 \mathrm{AQW}($ INODE $)=\mathrm{AQW}(\mathrm{INODE}) / \mathrm{AXN}$ (INODE)

GO TO 190

180 IF (AQ (INODE) .EQ. 0.) GO TO 190

$\mathrm{AQW}(\mathrm{INODE})=\mathrm{AQW}(\mathrm{INODE}) /(\mathrm{AQ}($ INODE $) * \mathrm{AXN}($ INODE $))$

190 CONTINUE

200 CALL SKMATK (AQ,AQW, ITITLE)

C

210 RETURN

END 
Attachment 7--FORTRAN SOURCE Code of Subroutines for Streamflow and Water-Quality Simulation--Continued

2350

2351

2352

2353

2354

2355

2356

2357

2358

2359

2360

2361

2362

2363

2364

2365

2366

2367

2368

2369

2370

2371

2372

2373

FUNCTION FINC (FMAX, FMIN)

C

RANGE = FMAX - FMIN

IF (RANGE . LT. . 1) GO TO 90

IFAC $=-10$

10 IFAC $=$ IFAC +1

IF (10.*IFAC - RANGE) $10,20,20$

20 FINC $=10 . *($ IFAC -1$)$

30 IMIN $=$ FMIN $/$ FINC

IF (FMIN) $40,60,60$

40 IF (FMIN/FINC +.05$) 50,60,60$

50 IMIN $=$ IMIN -1

60 IMAX $=$ FMAX $/$ FINC

IF (IMAX - IMIN - 4) $70,80,80$

70 FINC $=$ FINC $/ 2$.

GO TO 30

80 FMAX $=(\operatorname{IMAX}+1) *$ FINC

FMIN $=$ IMIN $*$ FINC

RETURN

90 FMAX $=$ FMAX +1 .

FMIN $=$ FMIN -1 .

FINC $=1$.

RETURN

END 
Attachment 7--FORTRAN SOURCE Code of Subroutines for Streamflow and Water-Quality Simulation--Continued

2374

2375

2376

2377

2378

2379

2380

2381

2382

2383

2384

2385

2386

2387

2388

2389
C

FUNCTION FUNCZ(X)

$B=1$.

$C=X$

IF $(C-.5) 20,20,10$

$10 B=-1$.

$\mathrm{C}=1 .-\mathrm{C}$

$20 \mathrm{C}=1 . /(C * \mathrm{C})$

$\mathrm{C}=\mathrm{ALOG}(\mathrm{C})$

$\mathrm{C}=\mathrm{SQRT}(\mathrm{C})$

$\mathrm{D}=2.511517+0.802853 * \mathrm{C}+0.010328 * \mathrm{C} * \mathrm{C}$

$E=1 .+1.432788 * C+0.189269 * C * C+0.001308 * C * C * C$

$C=C-D / E$

FUNCZ $=B * C$

RETURN

END 
Attachment 8--FORTRAN Source Code of Subroutines

for Water-Supply Operations Simulation

CBSNMD3

$\mathrm{C}$

$\mathrm{C}$

$\mathrm{C}$

MAINLINE OF RIVER BASIN SIMULATION MODEL (VERSION 非)

WRITTEN BY ALAN BURNS, U.S. GEOLOGICAL SURVEY WATER RESOURCES DIVISION, LAKEWOOD, CO LATEST UPDATE 5/21/87

$\mathrm{C}$

$\mathrm{C}$

COMMON NMONTH, NINDV , IN , IOUT , IPRINT , IGRAPH, IBATCH, IQW, IMONTH,

1 JMONTH, IYEAR

COMMON /NAMES/ TITL(5)

COMMON /REACHS/ NNODE, NODEID(100), NXTNOD(100),XLOC (100), YLOC (100), 1 PLTX (100), PLTY (100), NTRCPT $(100,12), \operatorname{SLOPE}(100,12)$,

2 QWA $(100,12), Q W B(100,12), \operatorname{INDEPV}(100,12), \mathrm{Q}(100)$,

3 QW(100), CONCFC (100), NODEUS (100), QSUBA (100),

REAL NTRCPT QWSUBA(100),DSA(100),DSB(100)

COMMON /RESPON/ FRSPNZ $(100,480,2), \operatorname{TLWATR}(100,2), \operatorname{TWCONC}(100,2)$

COMMON /SCHMTK/ ISKM,PORQ(100), PORQW(100), AQ(100), AQW(100),

1 QHYMAX (5), PORXN (100), AXN (100)

COMMON /SKMTKU/ ISKMGW, ISKMU, UMAX,ADD (100), AGW(100), ARR(100), 1 $\operatorname{AIM}(100), \operatorname{APR}(100), \operatorname{ACN}(100), \operatorname{ANEED}(100)$

C

DIMENSION ITITLE (20)

CHARACTER $\div 80$ IDUM

C

REAL ITITLE

EQUIVALENCE (IDUM, ITITLE)

C

IN $=1$

IOUT $=1$

C

WRITE (IOUT, 1000)

1000 FORMAT (54H IS THIS AN INTERACTIVE SESSION (0 FOR NO, 1 FOR YES)?) CALL QUESZN(IBATCH)

C

WRITE (IOUT, 1010)

1010 FORMAT $(44 \mathrm{H}$

$156 \mathrm{H}$

$240 \mathrm{H}$

BSNMD3 RIVER BASIN SIMULATION MODEL \#3 ,//,

C WRITTEN BY ALAN W. BURNS, USGS, LAKEWOOD, COLO , $/$, LATEST UPDATE MADE ON $1 / 28 / 87, / / /$ )

IF (IBATCH .EQ. 0) GO TO 40

WRITE (IOUT, 1020)

1020 FORMAT (53H FOR ALL QUESTIONS, 1 = YES AND ANY OTHER NUMBER = NO $1, /, 37 \mathrm{H}$ DO YOU WANT THE GENERAL EXPLANATION? )

CALL QUESZN(IQ)

IF (IQ-1) $20,10,20$

10 WRITE (IOUT, 1030)

1030 FORMAT $(/ /, 64 \mathrm{H}$

THIS MODEL IS THE THIRD IN A PLANNED SERIES OF 1 RIVER BASIN, /,63H SIMULATION MODELS. VERSION \#3 USES VERSION 非 2TO GENERATE THE, /,64H FLOW CONDITIONS OF THE BASIN. THE COMPLETE 3WATER USE SYSTEM IS, /,68H SIMULATED. WATER IS OBTAINED FROM VARIO 4US SOURCES IN A PRIORITIZED,/,60H LIST FOR EACH WATER USER TO SATI 5SFY PERCEIVED NEEDS. PRIOR,/,58H APPROPRIATION WATER LAWS, RESERV 6OIR STORAGE AND RELEASES, ,/,66H TRANSMOUNTAIN IMPORTS, AND GROUND7WATER PUMPAGE ARE ALL SIMULATED,/,49H ALONG WITH THE RETURN FLOWS 8OF UNCONSUMED WATER. ,///) 
Attachment 8--FORTRAN Source Code of Subroutines for Water-Supply Operations Simulation--Continued

93

94

95

96

20 WRITE (IOUT, 1040)

1040 FORMAT (30H DO YOU WANT THE INSTRUCTIONS? )

CALL QUESZN (IQ)

IF (IQ- 1$) \quad 40,30,40$

30 CALL INSTRK

CALL NSTRK2

C

40 IF (IBATCH .EQ. 1) CALL READFI

IF (IBATCH .NE. 1) CALL READFB

C

IF (IBATCH .EQ. 1) CALL READMI

IF (IBATCH .NE. 1) CALL READMB

IF (IBATCH .EQ. 1) CALL READUI

IF (IBATCH .NE. 1) CALL READUB

C

C

C

\section{INITIALIZATION}

DO 50 INODE $=1$, NNODE

PORQW (INODE) $=0$.

PORXN (INODE) $=0$.

PORQ (INODE) $=0$.

DO 50 ISIDE $=1,2$

TLWATR (INODE, ISIDE) $=0$.

50 TWCONC (INODE, ISIDE) $=0$.

DO 60 IUSER $=1$, NUSERS

$\mathrm{ADD}$ (IUSER) $=0$.

$A G W($ IUSER) $=0$.

$A R R$ (IUSER) $=0$.

$\operatorname{AIM}($ IUSER) $=0$.

$\operatorname{APR}($ IUSER $)=0$.

ANEED (IUSER) $=0$.

C

$60 \mathrm{ACN}$ (IUSER) $=0$.

CALL UNTRSP

CALL PRSTRS

IYEAR $=0$

C

IF (ISKM .NE. 1) GO TO 70

WRITE (IDUM, 1050) TITL

1050 FORMAT (5A4,22H - MONTHLY SIMULATION\$ )

CALL MNTHLY (ITITLE)

C

$\mathrm{C}$

$\mathrm{C}$

70 DO 110 IMONTH $=1$, NMONTH

JMONTH $=$ IMONTH $-(($ IMONTH -1$) / 12) \div 12$

IF (JMONTH-1) $80,80,100$

80 DO 90 INODE $=1$, NNODE

$\mathrm{AQW}($ INODE $)=0$.

$90 \mathrm{AQ}$ (INODE) $=0$.

IYEAR = IYEAR + 1

IF (IBATCH .EQ. 1 .AND. ISKM .NE. 1) WRITE (IOUT, 1060) IYEAR 
Attachment 8--FORTRAN Source Code of Subroutines

for Water-Supply Operations Simulation--Continued

109

110

111

112

113

114

115

116

117

118

119

120

121

122

123

124

125

126

127

128

129

130

131

132

133

134

135

136

137

138

139

140

141

142

143

144

145

146

147

148

149

150

151
1060 FORMAT (26H CURRENTLY SIMULATING YEAR, I3)

C

100 CALL NFLOW

CALL DEMAND

CALL ADJUST

CALL SUPPLY

CALL QUALTY

CALL SUMRYM

CALL SMRYMU

110 CONTINUE

C

C SUMMARIZE AND DO ANY DESIRED COMPARISONS

C

CALL SUMRYF

CALL SMRYFU

120 IF (IBATCH .EQ. 0) GO TO 130

WRITE (IOUT, 1070)

1070 FORMAT (71H DO YOU WANT TO COMPARE FLOW RESULTS FROM THIS RUN WITH 1 A PREVIOUS RUN? )

130 CALL QUESZN (IQ)

IF (IQ - 1) $150,140,150$

140 CALL CMPAR

GO TO 120

150 IF (IBATCH .EQ. 0) GO TO 160 WRITE (IOUT, 1080)

1080 FORMAT (71H DO YOU WANT TO COMPARE USER RESULTS FROM THIS RUN WITH 1 A PREVIOUS RUN? )

160 CALL QUESZN (IQ)

IF (IQ - 1) $180,170,180$

170 CALL CMPARU(1)

GO TO 150

180 IF (IBATCH .EQ. 0) GO TO 190

WRITE (IOUT, 1090)

1090 FORMAT (77H DO YOU WANT TO COMPARE RESERVOIR CONTENTS FROM THIS RU IN WITH A PREVIOUS RUN?)

190 CALL QUESZN (IQ)

IF (IQ - 1) $210,200,210$

200 CALL CMPARU(2)

GO TO 180

210 CONTINUE

IF (IGRAPH .NE. O) CALL DONEPL

STOP

END 
Attachment 8--FORTRAN Source Code of Subroutines for Water-Supply Operations Simulation--Continued

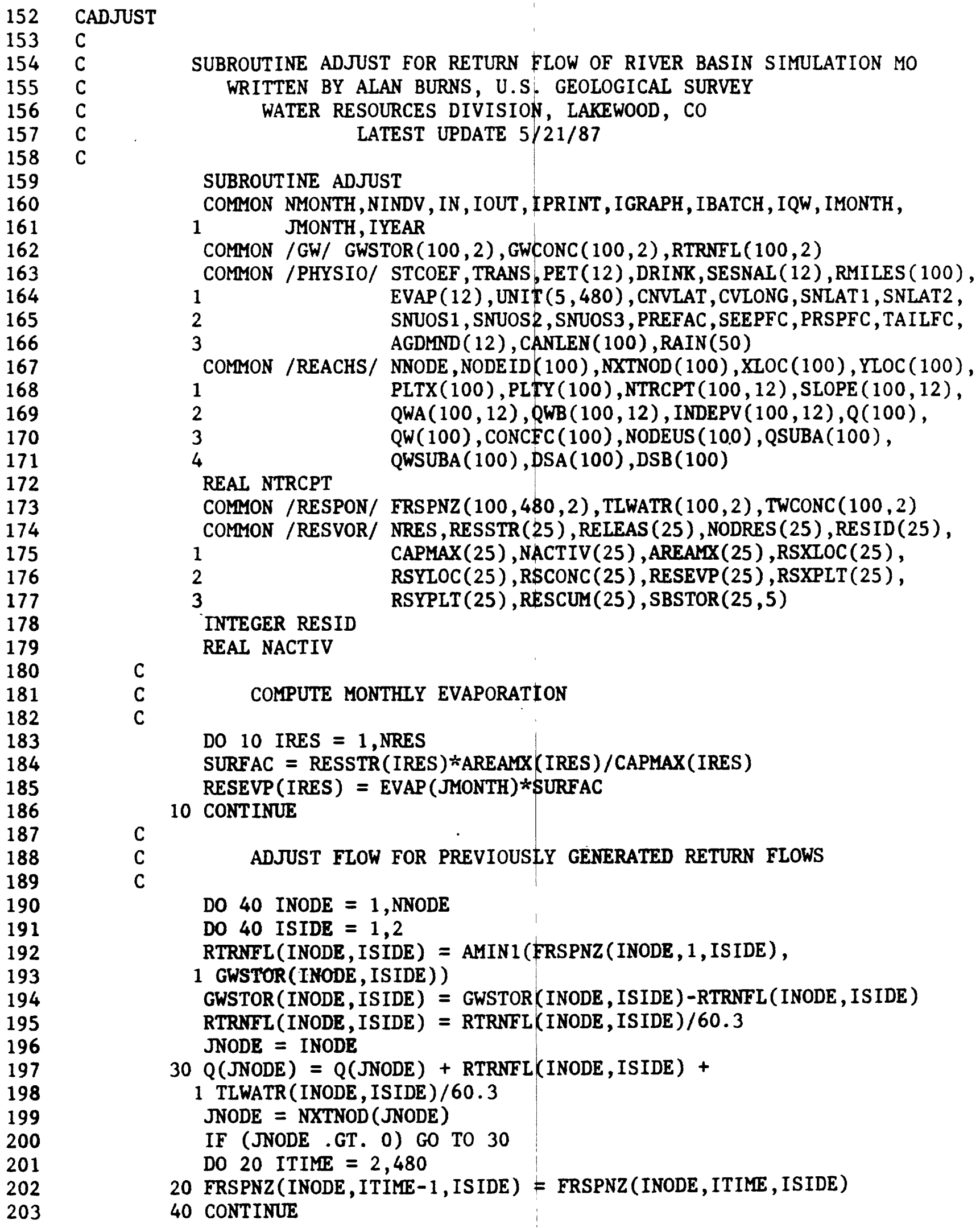

SUBROUTINE ADJUST FOR RETURN FLOW OF RIVER BASIN SIMULATION MO WRITTEN BY ALAN BURNS, U.S. GEOLOGICAL SURVEY WATER RESOURCES DIVISION, LAKEWOOD, CO LATEST UPDATE 5/21/87 
Attachment 8--FORTRAN Source Code of Subroutines

for Water-Supply Operations Simulation--Continued

204

205

206

207

208

209

210

211

212

213

214
C

C

C

NCARDS $=($ NINDV -1$) / 8+1$

DO $50 \mathrm{I}=1$, NCARDS

50 BACKSPACE (61)

$\operatorname{READ}(61,1000)(\operatorname{RAIN}(\mathrm{I}), \mathrm{I}=1, \mathrm{NINDV})$

1000 FORMAT (8F 10.2)

C

RETURN

END 
Attachment 8--FORTRAN Source Code of Subroutines

for Water-Supply Operations Simulation--Continued

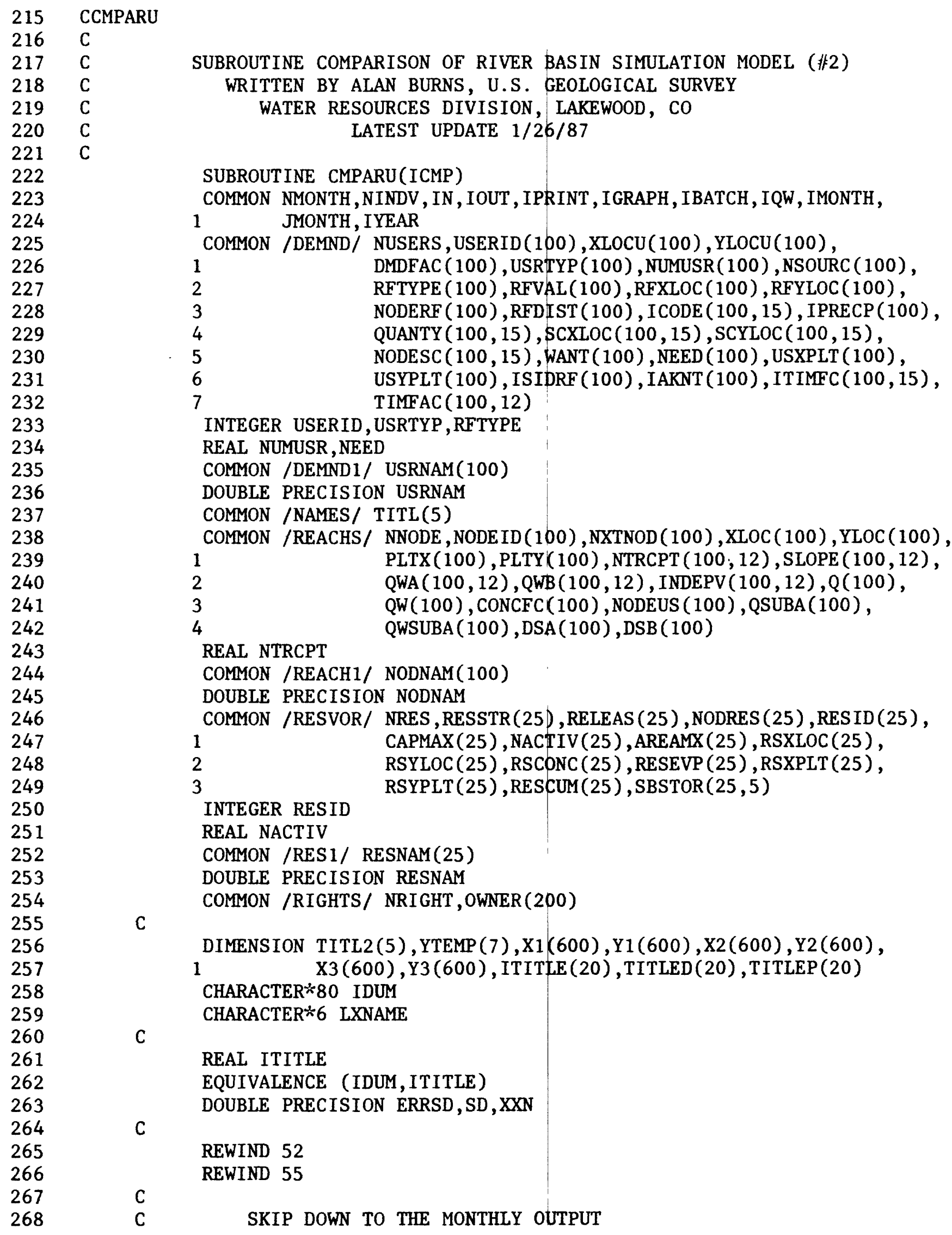

C

SUBROUTINE COMPARISON OF RIVER BASIN SIMULATION MODEL (非)

WRITTEN BY ALAN BURNS, U.S. GEOLOGICAL SURVEY WATER RESOURCES DIVISION, LAKEWOOD, CO LATEST UPDATE $1 / 26 / 87$

SUBROUTINE CMPARU(ICMP)

COMMON NMONTH, NINDV, IN, IOUT , IPRINT , I GRAPH, IBATCH, IQW, IMONTH, 1 JMONTH, IYEAR

COMMON /DEMND/ NUSERS, USERID (100), XLOCU(100), YLOCU(100), $\begin{array}{ll}1 & \text { DMDFAC }(100), \operatorname{USRTYP}(100), \operatorname{NUMUSR}(100), \operatorname{NSOURC}(100), \\ 2 & \operatorname{RFTYPE}(100), \operatorname{RFVAL}(100), \operatorname{RFXLOC}(100), \operatorname{RFYLOC}(100), \\ 3 & \text { NODERF }(100), \operatorname{RFDIST}(100), \operatorname{ICODE}(100,15), \operatorname{IPRECP}(100), \\ 4 & \text { QUANTY }(100,15), \operatorname{SCXLOC}(100,15), \operatorname{SCYLOC}(100,15), \\ 5 & \text { NODESC }(100,15), \operatorname{WANT}(100), \operatorname{NEED}(100), \operatorname{USXPLT}(100), \\ 6 & \text { USYPLT }(100), \operatorname{ISIDRF}(100), \operatorname{IAKNT}(100), \operatorname{ITIMFC}(100,15), \\ 7 & \text { TIMFAC }(100,12) \\ \text { INTEGER USERID, USRTYP , RFTYPE }\end{array}$

REAL NUMUSR, NEED

COMMON /DEMND1/ USRNAM(100)

DOUBLE PRECISION USRNAM

COMMON /NAMES/ TITL(5)

COMMON /REACHS/ NNODE, NODEID (100), NXTNOD (100), XLOC (100), YLOC (100), 1

2

3

4

REAL NTRCPT $\operatorname{PLTX}(100), \operatorname{PLTY}(100), \operatorname{NTRCPT}(100,12), \operatorname{SLOPE}(100,12)$, COMMON /REACH1/ NODNAM(100)

DOUBLE PRECISION NODNAM

COMMON /RESVOR/ NRES, RESSTR(25), RELEAS (25), NODRES (25), RESID (25), 1

2 CAPMAX (25), NACTIV (25), AREAMX (25), RSXLOC (25),

3

INTEGER RESID

REAL NACTIV

COMMON /RES1/ RESNAM(25)

DOUBLE PRECISION RESNAM

COMMON /RIGHTS/ NRIGHT, OWNER (200)

DIMENSION TITL2(5), YTEMP (7), X1 (600), Y1(600),X2(600), Y2(600), $1 \mathrm{X} 3(600), \mathrm{Y} 3(600), \operatorname{ITITLE}(20), \operatorname{TITLED}(20), \operatorname{TITLEP}(20)$

C CHARACTER $* 80$ IDUM

CHARACTER $* 6$ LXNAME

REAL ITITLE

EQUIVALENCE (IDUM, ITITLE)

C

DOUBLE PRECISION ERRSD, SD, XXN

REWIND 52

REWIND 55

C

C

SKIP DOWN TO THE MONTHLY OUTPUT 
Attachment 8--FORTRAN Source Code of Subroutines

for Water-Supply Operations Simulation--Continued

C

READ $(52,1000)$ ITEST, ITEST, ITEST, ITEST

READ $(55,1000)$ ITEST, ITEST, ITEST

1000 FORMAT (1A1)

READ $(55,1010)$ TITL2

1010 FORMAT (20X,5A4)

WRITE (IOUT, 1020) TITL2

1020 FORMAT (//47H THE PREVIOUS RUN YOU LOADED FOR COMPARISON IS: ,5A4)

READ $(52,1000)$ ITEST, ITEST, ITEST

READ $(55,1000)$ ITEST, ITEST

READ $(55,1030)$ TITLED, NNODEP

1030 FORMAT $(37 \mathrm{X}, 20 \mathrm{~A} 4,6 \mathrm{X}, \mathrm{I3})$

WRITE (IOUT, 1040) TITLED

1040 FORMAT (43H ITS ADDITIONAL BASIN DESCRIPTION FILE WAS: ,/,20A4)

DO $10 \mathrm{I}=1,41$

READ $(52,1000)$ ITEST

10 READ $(55,1000)$ ITEST

READ $(52,1050)$ NRES

READ $(55,1050)$ NRESP

1050 FORMAT $(10 \mathrm{X}, \mathrm{I} 4)$

READ $(52,1000)$ ITEST, ITEST, ITEST, ITEST, ITEST

READ $(55,1000)$ ITEST, ITEST, ITEST, ITEST, ITEST

DO $20 \mathrm{I}=1$, NRES

20 READ $(52,1000)$ ITEST

DO $30 I=1$, NRESP

30 READ $(55,1000)$ ITEST

READ $(52,1000)$ ITEST, ITEST, ITEST , ITEST , ITEST

READ $(55,1000)$ ITEST, ITEST, ITEST, ITEST, ITEST

DO $40 \mathrm{I}=1$, NNODE

$40 \operatorname{READ}(52,1000)$ ITEST

DO $50 \mathrm{I}=1$, NNODEP

50 READ $(55,1000)$ ITEST

READ $(52,1000)$ ITEST

READ $(55,1060)$ TITLED, NUSERP

1060 FORMAT $(18 \mathrm{X}, 20 \mathrm{~A} 4,22 \mathrm{X}, \mathrm{I} 3)$

WRITE (IOUT, 1070) TITLED

1070 FORMAT (25H ITS WATER USER FILE WAS: ,/,20A4)

READ $(52,1000)$ ITEST, ITEST, ITEST, ITEST, ITEST , ITEST , ITEST , ITEST

READ $(55,1000)$ ITEST, ITEST, ITEST, ITEST, ITEST , ITEST , ITEST, ITEST

DO $60 \mathrm{I}=1$, NUSERS

READ $(52,1080)$ NSORC

1080 FORMAT $(90 \mathrm{X}, \mathrm{I} 2)$

DO 60 LINES $=1$, NSORC

60 READ $(52,1000)$ ITEST

DO $70 \mathrm{I}=1$, NUSERP

READ $(55,1080)$ NSORC

DO 70 LINES $=1$, NSORC

70 READ $(55,1000)$ ITEST

READ $(52,1000)$ ITEST

READ $(55,1090)$ TITLED, NRIGTP

1090 FORMAT $(19 \mathrm{X}, 20 \mathrm{~A} 4,20 \mathrm{X}, \mathrm{I3})$

WRITE (IOUT, 1100) TITLED

1100 FORMAT (26H ITS WATER RIGHT FILE WAS: ,/,20A4)

READ $(52,1000)$ ITEST, ITEST, ITEST, ITEST

READ $(55,1000)$ ITEST, ITEST, ITEST, ITEST

DO $80 \mathrm{I}=1$, NRIGHT 
Attachment 8--FORTRAN Source Code of Subroutines

for Water-Supply Operations Simulation--Continued

80 READ $(52,1000)$ ITEST

DO $90 \mathrm{I}=1$, NRIGTP

90 READ $(55,1000)$ ITEST

C

$$
\begin{aligned}
& \text { IUSER }=-99 \\
& \text { IRES }=-99 \\
& \text { IF (ICMP .EQ. 2) GO TO } 210
\end{aligned}
$$

\section{COMPARE WATER USER DATA}

IF (IBATCH .EQ. 0) GO TO 100

WRITE (IOUT, 1110)

1110 FORMAT (27H ENTER THE USER ID (5 COLS) )

100 READ (IN, 1120) IUSER

1120 FORMAT (I5, A8, 2X, 2F 10.0, I5 , 3F 10.0)

DO 110 IIUSER $=1$, NUSERS

IF (USERID(IIUSER) .NE. IUSER) GO TO 110 GO TO 120

110 CONTINUE

120 IF (IBATCH .EQ. 0) GO TO 130 WRITE (IOUT, 1130)

1130 FORMAT (17H ENTER 1 FOR NEED ,/,7X,22H2 FOR DIRECT DIVERSION ,/,7X $1,13 \mathrm{H} 3$ FOR PUMPAGE ,/,7X,24H4 FOR RESERVOIR RELEASES ,/,7X,

2 13H5 FOR IMPORTS ,/,7X,19H6 FOR TOTAL APPLIED ,/,7X,

$320 \mathrm{H} 7$ FOR TOTAL CONSUMED )

130 READ (IN, 1140) ITYPE

1140 FORMAT (I1) GO TO $(140,150,160,170,180,190,200)$, ITYPE

140 WRITE (IOUT, 1150) USERID(IIUSER), USRNAM(IIUSER)

1150 FORMAT (4OH THE WATER NEED, IN ACRED-FEET, FOR USER , I5 , 2H (, A8, 1 14H) ARE COMPARED ) GO TO 250

150 WRITE (IOUT, 1160) USERID (IIUSER), USRNAM(IIUSER)

1160 FORMAT (46H THE DIRECT DIVERSIONS, IN ACRE-FEET, FOR USER , I5, 2H ( $1, \mathrm{~A} 8,14 \mathrm{H})$ ARE COMPARED )

GO TO 250

160 WRITE (IOUT, 1170) USERID(IIUSER), USRNAM(IIUSER)

1170 FORMAT (37H THE PUMPAGE, IN ACRE-FEET, FOR USER , I5, 2H (, A8, 1 14H) ARE COMPARED )

GO TO 250

170 WRITE (IOUT, 1180) USERID (IIUSER), USRNAM(IIUSER)

1180 FORMAT (48H THE RESERVOIR RELEASES, IN ACRE-FEET, FOR USER , I5, $12 \mathrm{H}(, \mathrm{A} 8,14 \mathrm{H})$ ARE COMPARED $)$ GO TO 250

180 WRITE (IOUT, 1190) USERID (IIUSER), USRNAM(IIUSER)

1190 FORMAT (37H THE IMPORTS, IN ACRE-FEET, FOR USER , I5, 2H (, A8,

1 14H) ARE COMPARED )

GO TO 250

190 WRITE (IOUT, 1200) USERID (IIUSER), USRNAM(IIUSER)

1200 FORMAT (48H THE TOTAL APPLIED WATER, IN ACRE-FEET, FOR USER , I5,

$12 \mathrm{H}(, \mathrm{A} 8,14 \mathrm{H})$ ARE COMPARED ) GO TO 250

200 WRITE (IOUT, 1210) USERID (IIUSER), USRNAM(IIUSER)

1210 FORMAT (48H THE TOTAL WATER CONSUMED, IN ACRE-FEET, BY USER , I5, $12 \mathrm{H}(, \mathrm{A} 8,14 \mathrm{H})$ ARE COMPARED ) GO TO 250 
Attachment 8--FORTRAN Source Code of Subroutines

for Water-Supply Operations Simulation--Continued

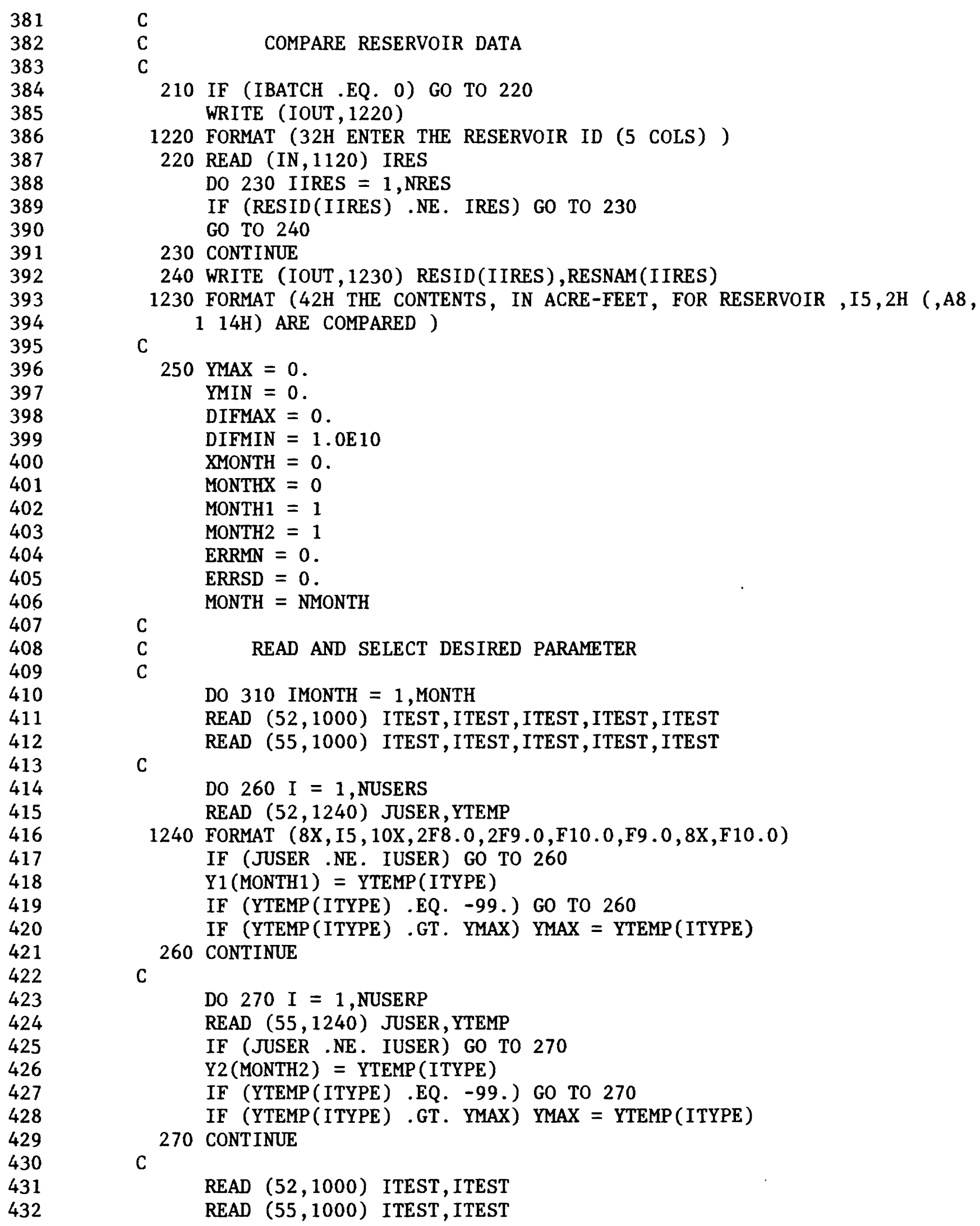

C

C COMPARE RESERVOIR DATA

C

210 IF (IBATCH .EQ. 0) GO TO 220

WRITE (IOUT, 1220)

1220 FORMAT (32H ENTER THE RESERVOIR ID (5 COLS) )

220 READ (IN, 1120) IRES

DO 230 IIRES $=1$, NRES

IF (RESID(IIRES) .NE. IRES) GO TO 230

GO TO 240

230 CONTINUE

240 WRITE (IOUT , 1230) RESID(IIRES), RESNAM(IIRES)

1230 FORMAT (42H THE CONTENTS, IN ACRE-FEET, FOR RESERVOIR , I5, 2H (, A8,

C

1 14H) ARE COMPARED ) 
Attachment 8--FORTRAN Source Code of Subroutines for Water-Supply Operations Simulation--Continued

C

READ $(52,1000)$ ITEST, ITEST, ITEST , ITEST, ITEST

READ $(55,1000)$ ITEST, ITEST, ITEST, ITEST, ITEST

DO $280 \mathrm{I}=1$, NRES

READ $(52,1250)$ JRES, YTEMP (1)

1250 FORMAT $(13 \mathrm{X}, \mathrm{I} 6,13 \mathrm{X}, \mathrm{F} 11.0)$

IF (JRES .NE. IRES) GO TO 280

Y1 (MONTH1) = YTEMP $(1)$

IF (YTEMP (1) .EQ. -99.) GO TO 280

IF (YTEMP (1) .GT. YMAX) YMAX = YTEMP (1)

280 CONTINUE

DO $290 \mathrm{I}=1$, NRESP

READ $(55,1250)$ JRES,YTEMP (1)

IF (JRES . NE. IRES) GO TO 290

Y2 $($ MONTH2) $=$ YTEMP $(1)$

IF (YTEMP (1) .EQ. -99.) GO TO 290

IF (YTEMP (1) .GT. YMAX) YMAX = YTEMP (1)

290 CONTINUE

C

$\mathrm{X} 1$ (MONTH1) $=$ IMONTH

$\mathrm{X} 1($ MONTH 1$)=\mathrm{X} 1$ (MONTH 1$)-.25$

$\mathrm{X} 2($ MONTH 2$)=\mathrm{X} 1($ MONTH 1$)+.5$

IF (Y1(MONTH1) .EQ. -99.) GO TO 300

MONTH $1=$ MONTH $1+1$

IF (Y2(MONTH2) .EQ. -99.) GO TO 310

MONTH2 $=$ MONTH2 +1

$\mathrm{ERR}=\mathrm{Y} 1($ MONTH1 - 1) - Y2(MONTH2 - 1)

IF (ERR .GT. DIFMAX) DIFMAX = ERR

IF (ERR . LT. DIFMIN) DIFMIN $=$ ERR

XMONTH $=$ XMONTH +1 .

MONTHX $=$ MONTHX +1

$\mathrm{X} 3($ MONTHX $)=$ IMONTH

Y3 $($ MONTHX) $=$ ERR

$\mathrm{ERRMN}=\mathrm{ERRMN}+\mathrm{ERR}$

ERRSD $=E R R S D+E R R \div E R R$

GO TO 310

300 IF (Y2(MONTH2) .EQ. -99.) GO TO 310

MONTH2 $=$ MONTH2 +1

310 CONTINUE

C

IF (XMONTH .LE. 1.) RETURN

MONTH1 $=$ MONTH $1-1$

MONTH2 $=$ MONTH2 -1

SD = ERRMN*ERRMN $/$ XMONTH

$\mathrm{XXN}=\mathrm{XMONTH}-1$.

ERRSD $=\operatorname{DSQRT}(($ ERRSD $-\mathrm{SD}) / \mathrm{XXN})$

ERRMN $=$ ERRMN $/$ XMONTH

WRITE (IOUT, 1260) ERRMN, ERRSD

1260 FORMAT ( 43 H THE MEAN RESIDUAL BETWEEN THE TWO RUNS IS ,F10.3, $1 /, 30 \mathrm{H}$ THE STANDARD DEVIATION IS ,F10.3)

IF (IGRAPH .EQ. O) RETURN

C

C 
Attachment 8--FORTRAN Source Code of Subroutines for Water-Supply Operations Simulation--Continued

486

487

488

489

490

491

492

493

494

495

496

497

498

499

500

501

502

503

504

505

506

507

508

509

510

511

512

513

514

515

516

517

518

519

520

521

522

523

524

525
C

XMIN $=0$.

$\mathrm{XMAX}=$ MONTH +1

IF (MONTH .GT. 37) XMAX $=$ XMAX/12.

$\mathrm{XINC}=\mathrm{FINC}(\mathrm{XMAX}, \mathrm{XMIN})$

IF (MONTH .GT. 37) XMAX $=$ XMAX $* 12$.

YINC $=$ FINC $($ YMAX, YMIN $)$

CALL RESET ('ALL')

CALL PAGE $(11 ., 8.5)$

CALL NOBRDR

CALL AREA2D $(10,6.0)$

CALL INTAXS

C

CALL HEIGHT (.2)

LXNAME $=$ 'MONTHS'

IF (MONTH .GT. 37) LXNAME = 'YEARS '

GO TO $(320,400)$ ICMP

320 WRITE (IDUM, 1270) USERID(IIUSER), USRNAM(IIUSER)

1270 FORMAT $(\mathrm{I} 5,2 \mathrm{H}(, \mathrm{A} 8,2 \mathrm{H}) \$)$

CALL HEADIN (ITITLE, 100, $1 ., 1$ )

CALL XNAME (LXNAME , 6)

GO TO $(330,340,350,360,370,380,390)$, ITYPE

330 CALL YNAME (24HWATER NEED, IN ACRE-FEET, 24)

GO TO 410

340 CALL YNAME (31HDIRECT DIVERSIONS, IN ACRE-FEET, 31)

GO TO 410

350 CALL YNAME (21HPUMPAGE, IN ACRE-FEET, 21)

GO TO 410

360 CALL YNAME (32HRESERVOIR RELEASES, IN ACRE-FEET, 32)

GO TO 410

370 CALL YNAME (21HIMPORTS, IN ACRE-FEET, 21)

GO TO 410

380 CALL YNAME (33HTOTAL APPLIED WATER, IN ACRE-FEET, 33)

GO TO 410

390 CALL YNAME (34HTOTAL WATER CONSUMED, IN ACRE-FEET, 34)

GO TO 410

400 WRITE (IDUM, 1270) RESID(IIRES), RESNAM(IIRES)

CALL HEADIN (ITITLE, $100,1 ., 1$ )

CALL XNAME (LXNAME, 6)

CALL YNAME (3OHSTORAGE CONTENTS, IN ACRE-FEET, 30) 
Attachment 8--FORTRAN Source Code of Subroutines for Water-Supply Operations Simulation--Continued

410 CALL XTICKS $(0)$

CALL YTICKS $(0)$

CALL XNONUM

CALL GRAF (XMIN, XINC, XMAX, YMIN, YINC, YMAX)

CALL BARS $(-.25)$

CALL SETCLR(3HRED)

CALL MESSAG(TITL, 20,4.0,5.7)

CALL CURVE (X1, Y1, MONTH 1,0$)$

CALL SETCLR (5HGREEN)

CALL MESSAG(TITL2, 20,4.0,5.4)

CALL CURVE (X2, Y2, MONTH2,0)

IF (MONTH .GT. 37) XMAX $=$ XMAX/12.

CALL SETCLR (5HWHITE)

CALL RESET ('XNONUM')

CALL XGRAXS (XMIN, XINC, XMAX, 10, $1 \mathrm{H}, 1,0 ., 0$.

ITICKS $=$ XINC +.1

CALL XTICKS (ITICKS)

CALL RESET ('YTICKS')

CALL XNONUM

CALL YNONUM

CALL XGRAXS (XMIN, XINC, XMAX, 10, ,1H ,-1,0.,0.)

CALL YGRAXS (YMIN, YINC, YMAX, 6.0,1H ,-1,0.,0.)

CALL XGRAXS (XMIN,XINC, XMAX, 10, 1H ,1,0.,6.0)

CALI YGRAXS (YMIN, YINC, YMAX, 6.0,1H ,1,10.,0.)

CALL ENDPL(0)

C

C

C

PLOT THE TWO SETS OF DATA AS FREQUENCY CURVES

CALL SORT (Y1, MONTH1)

CALL PROB (X1,MONTH 1$)$

CALL SORT (Y2, MONTH2)

CALL PROB (X2, MONTH2)

CALL RESET ('ALL')

CALL PAGE $(11,8.5)$

CALL NOBRDR

CALL AREA2D $(10,6.0)$

CALL INTAXS

C

CALL HEIGHT (.2)

CALL HEADIN (ITITLE, $100,1 ., 1$ )

CALL XNAME (26HPERCENT OF MONTHS EXCEEDED ,26)

GO TO $(420,500)$, ICMP

420 GO TO $(430,440,450,460,470,480,490)$, ITYPE

430 CALL YNAME (24HWATER NEED, IN ACRE-FEET , 24)

GO TO 510

440 CALL YNAME (31HDIRECT DIVERSIONS, IN ACRE-FEET , 31) GO TO 510

450 CALL YNAME (21HPUMPAGE, IN ACRE-FEET, 21) GO TO 510

460 CALL YNAME (32HRESERVOIR RELEASES, IN ACRE-FEET , 32) GO TO 510

470 CALL YNAME (21HIMPORTS, IN ACRE-FEET, 21) GO TO 510 
Attachment 8--FORTRAN Source Code of Subroutines for Water-Supply Operations Simulation--Continued

480 CALL YNAME (33HTOTAL APPLIED WATER, IN ACRE-FEET,33) GO TO 510

490 CALL YNAME (34HTOTAL WATER CONSUMED, IN ACRE-FEET, 34) GO TO 510

500 CALL YNAME (30HSTORAGE CONTENTS, IN ACRE-FEET, 30)

510 XMIN $=X 1(1)$

IF $(X 2(1)$.GT. XMIN) $X M I N=X 2(1)$

$\mathrm{XMAX}=\mathrm{X} 1(\mathrm{MONTH} 1)$

IF (X2 (MONTH2) .LT. XMAX) XMAX $=$ X2(MONTH2)

$\mathrm{XINC}=\mathrm{XMAX}-\mathrm{XMIN}$

CALL HEIGHT (.2)

CALL XNONUM

CALL XTICKS $(0)$

CALL YTICKS $(0)$

CALL GRAF (XMIN, XINC, XYAX, YMIN, YINC, YMAX)

CALL SETCLR (3HRED)

CALL MESSAG(TITL , 20, 2.5,5.7)

CALL CURVE (X1, Y1, MONTH 1,0$)$

CALL HWSHD

CALL SHDPAT ( 1 )

CALL SHDCRV (X1, Y1, MONTH $1, \mathrm{X} 1, \mathrm{Y} 1,5$ HBELOW)

CALL SETCLR (5HGREEN)

CALL MESSAG (TITL2, 20,2.5,5.4)

CALL CURVE (X2, Y 2, MONTH 2,0$)$

CALL RESET ('HWSHD')

CALL SHDPAT (3)

CALL SHDCRV (X2, Y2, MONTH2, X2, Y2, 5HBELOW)

CALL SETCLR (5HWHITE)

CALL RESET ('YTICKS')

CALL YNONUM

CALL RLVEC (XMIN, YMIN, XMAX, YMIN , 0)

CALL YGRAXS (YMIN, YINC, YMAX, 6.0,1H , -1,0.,0.)

CALL RLVEC (XMIN, YMAX, XMAX, YMAX, 0)

CALL YGRAXS (YMIN, YINC, YMAX, 6.0,1H ,1,10.,0.)

MONTH = MONTH 1

IF (MONTH2 . GT. MONTH) MONTH $=$ MONTH2

CALL RKRLAB (MONTH, YMAX, YMIN)

$\mathrm{C}$

CALL ENDPL $(0)$

C

PLOT A HYDROGRAPH OF THE RESIDUALS

MONTH $=$ NMONTH

IF (MONTHP .LT . MONTH) MONTH = MONTHP

$X M I N=0$.

$\mathrm{XMAX}=\mathrm{NMONTH}+1$

IF (MONTH .GT. 37) XMLAX $=$ XMAX/12.

$\mathrm{XINC}=\mathrm{FINC}(\mathrm{XMAX}, \mathrm{XMIN})$

IF (MONTH .GT. 37) XILAX $=X M A X * 12$.

DIFINC $=$ FINC (DIFMAX, DIFMIN $)$

CALL RESET ('ALL')

CALL PAGE $(11,, 8.5)$

CALL NOBRDR

CALL AREA2D $(10,6.0)$

CALL INTAXS 
Attachment 8--FORTRAN Source Code of Subroutines

for Water-Supply Operations Simulation--Continued

634

635

636

637

638

639

640

641

642

643

644

645

646

647

648

649

650

651

652

653

654

655

656

657

658

659

660

661

662

663

664

665

666

667

668

669

670

671

672

673

674

675

676

677

678

679

680

681

682

683
CALL HEIGHT $(.2)$

CALL HEADIN (ITITLE, $100,1 ., 1$ )

CALL XNAME $(1 \mathrm{H}, 1)$

GO TO $(520,600)$ ICMP

520 GO TO $(530,540,550,560,570,580,590)$, ITYPE

530 CALL YNAME (24HWATER NEED, IN ACRE-FEET, 24)

GO TO 610

540 CALL YNAME (31HDIRECT DIVERSIONS, IN ACRE-FEET , 31)

GO TO 610

550 CALL YNAME (21HPUMPAGE, IN ACRE-FEET , 21)

GO TO 610

560 CALL YNAME (32HRESERVOIR RELEASES, IN ACRE-FEET, 32)

GO TO 610

570 CALL YNAME (21HIMPORTS, IN ACRE-FEET ,21)

GO TO 610

580 CALL YNAME (33HTOTAL APPLIED WATER, IN ACRE-FEET, 33)

GO TO 610

590 CALL YNAME (34HTOTAL WATER CONSUMED, IN ACRE-FEET, 34)

GO TO 610

600 CALL YNAME (30HSTORAGE CONTENTS, IN ACRE-FEET, 30)

610 CALL CROSS

CALL HEIGHT (.2)

CALL XNONUM

CALL XTICKS (0)

CALI YTICKS $(0)$

CALL GRAF (XMIN, XINC, XMAX, DIFMIN, DIFINC,DIFMAX)

CALL MESSAG(TITL, 20,2.0,6.1)

CALL MESSAG(TITL2, 20,6.0,6.1)

CALL SETCLR (4HCYAN)

CALL BARPAT (16)

CALL BARWID $(-1$.

CALL VBARS (X3, Y3, 'BASE', MONTHX)

IF (MONTH .GT . 37) XMAX $=$ XMAX/12.

CALL SETCLR(5HWHITE)

ITICKS $=$ XINC +.1

CALI XTICKS (ITICKS)

CALL RESET ('YTICKS')

CALI YNONUM

CALL XGRAXS (XMIN, XINC, XMAX, 10.,1H ,-1,0.,0.)

CALL YGRAXS (DIFMIN,DIFINC,DIFMAX, 6.0,1H ,-1,0.,0.)

CALL XGRAXS (XMIN,XINC, XMAX, 10.,1H ,1,0.,6.0)

CALL YGRAXS (DIFMIN, DIFINC,DIFMAX , 6.0,1H , 1, 10.,0.)

CALL RESET ('XNONUM')

CALL XTICKS $(0)$

IF (MONTH.LT. 38) CALL XGRAXS (XMIN, XINC, XMAX, 10 , 6HMONTHS , 6, 0 . ,0.)

IF (MONTH.GT.37) CALL XGRAXS (XMIN, XINC, XMAX, 10.,5HYEARS ,5, 0.,0.)

C

CALL ENDPL (0)

RETURN

END 
Attachment 8--FORTRAN Source Code of Subroutines

for Water-Supply Operations Simulation--Continued

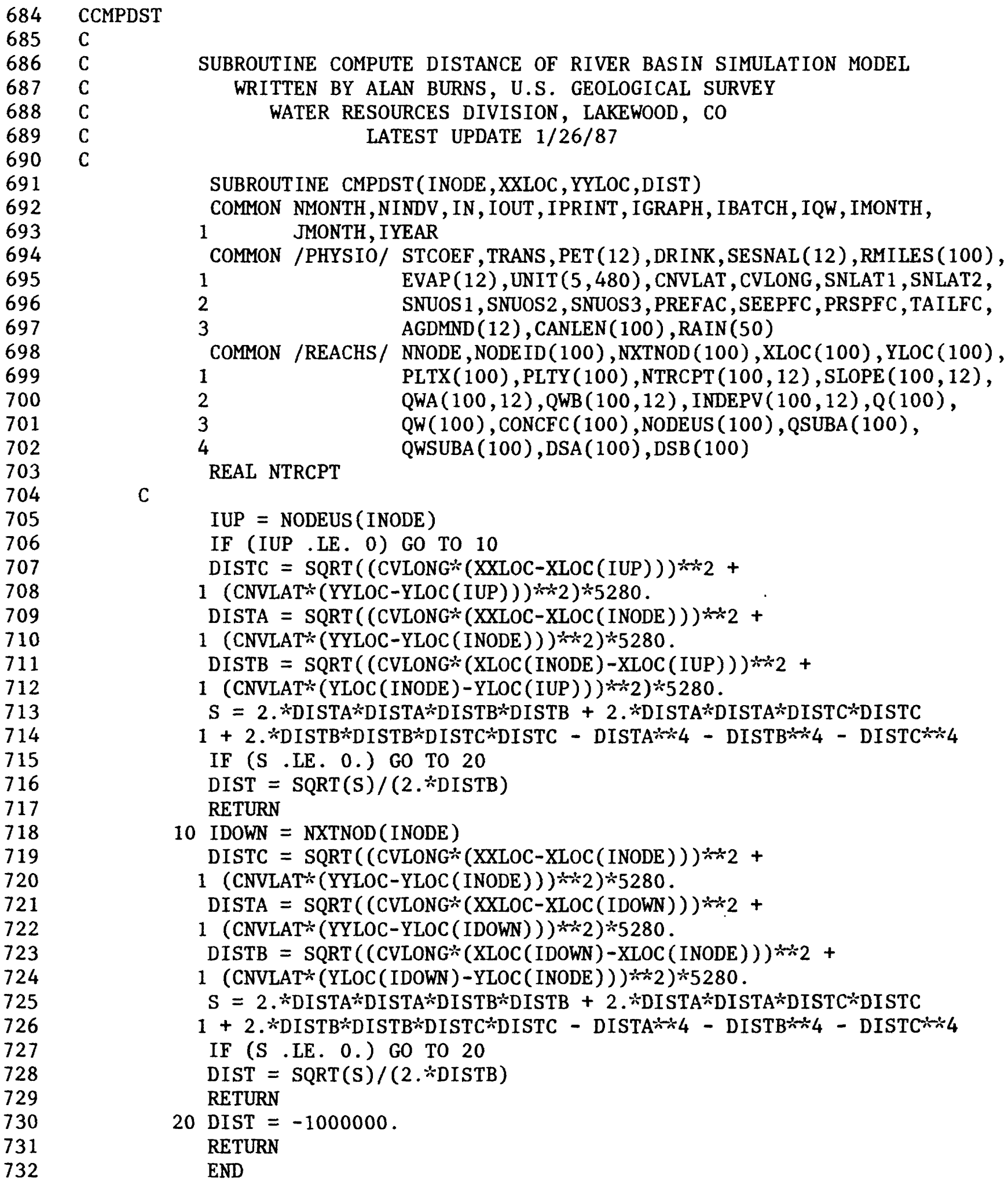


Attachment 8--FORTRAN Source Code of Subroutines for Water-Supply Operations Simulation--Continued

733 CCMPUTR

734 C

$735 \quad C$

736 C

$737 \quad \mathrm{C}$

$738 \quad \mathrm{C}$

739 C

$740 \quad \mathrm{C}$

741

742

743

744

745

746

747

748

749

750

751

752

753

754

755

756

757

758

759

760

761

762

763

C

C

C
SUBROUTINE COMPUTE FUTURE RESPONSES OF RIVER BASIN SIMULATION MODEL

WRITTEN BY ALAN BURNS, U.S. GEOLOGICAL SURVEY

WATER RESOURCES DIVISION, LAKEWOOD, CO

LATEST UPDATE $1 / 23 / 87$

SUBROUTINE CMPUTR(STRESS, NODE, ISIDE, ADIST)

COMMON /PHYSIO/ STCOEF, TRANS,PET(12),DRINK, SESNAL (12), RMILES (100), 1

2

3 $\operatorname{EVAP}(12), \operatorname{UNIT}(5,480)$, CNVLAT , CVLONG , SNLAT 1, SNLAT2, SNUOS 1 , SNUOS 2 , SNUOS3, PREFAC , SEEPFC, PRSPFC, TAILFC, AGDMND (12), CANLEN (100), RAIN (50)

COMMON /RESPON/ $\operatorname{FRSPNZ}(100,480,2), \operatorname{TLWATR}(100,2), \operatorname{TWCONC}(100,2)$

DIMENSION DIST(5)

DATA DIST $/ 3.0,3.3,3.7,4.0,4.3 /$

IF (STRESS .EQ. 0.) RETURN

IF (ADIST .EQ. 0.) ADIST $=100$.

BDIST $=$ ALOG10 (ADIST)

DO $10 \mathrm{I}=1,5$

IF (DIST(I) .GT. BDIST) GO TO 20

10 CONTINUE

$I=5$

20 DO 30 ITIME $=1,480$

30 FRSPNZ(NODE, ITIME, ISIDE) = FRSPNZ (NODE, ITIME, ISIDE) + STRESS *

1 UNIT ( I, ITIME)

RETURN

END 
Attachment 8--FORTRAN Source Code of Subroutines

for Water-Supply Operations Simulation--Continued

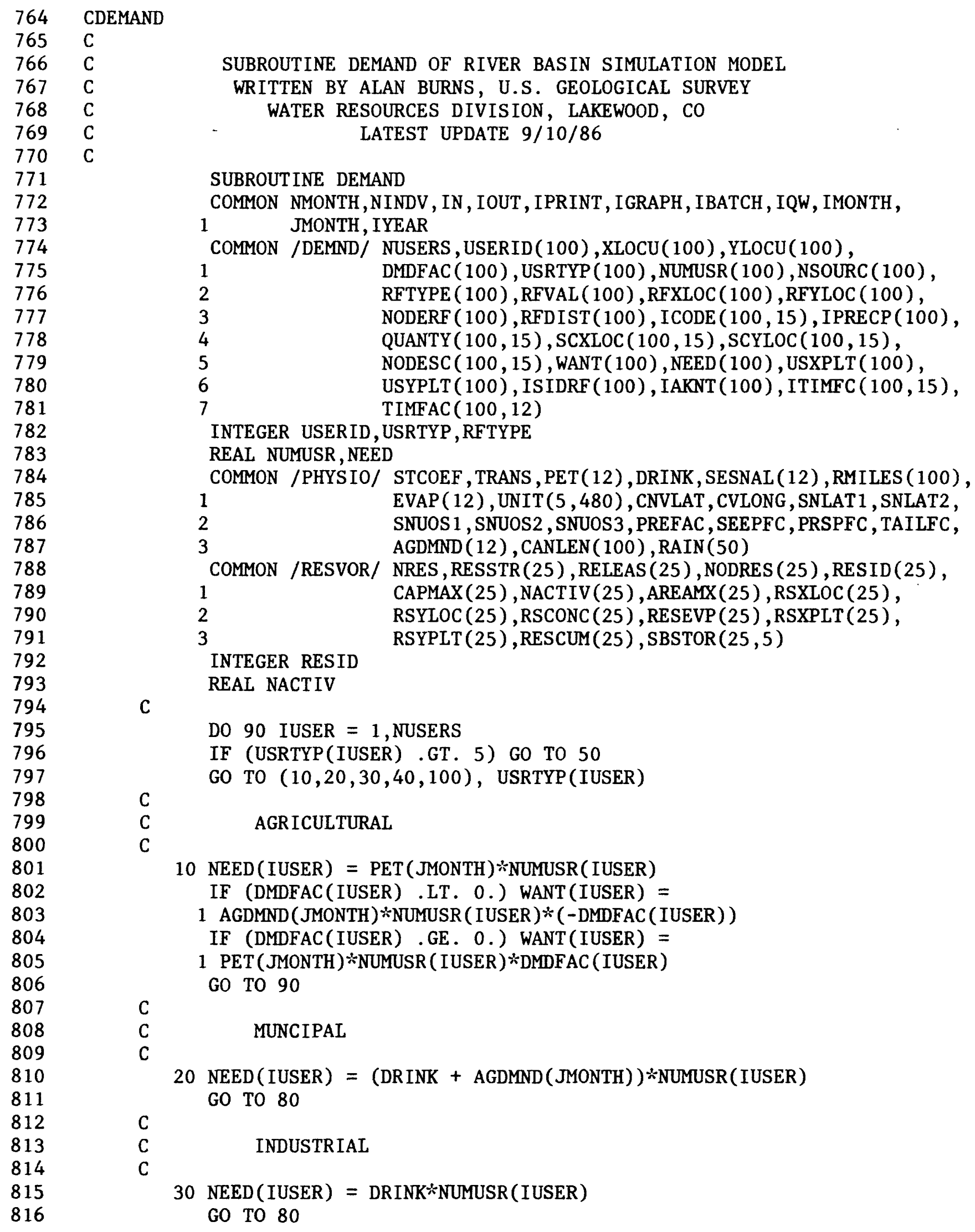

SUBROUTINE DEMAND OF RIVER BASIN SIMULATION MODEL WRITTEN BY ALAN BURNS, U.S. GEOLOGICAL SURVEY WATER RESOURCES DIVISION, LAKEWOOD, CO LATEST UPDATE $9 / 10 / 86$

SUBROUTINE DEMAND

COMMON NMONTH, NINDV, IN, IOUT, IPRINT, I GRAPH, IBATCH, IQW, IMONTH, 1 JMONTH, IYEAR

COMMON /DEMND/ NUSERS, USERID(100), XLOCU(100), YLOCU(100),

$1 \quad \operatorname{DMDFAC}(100), \operatorname{USRTYP}(100), \operatorname{NUMUSR}(100), \operatorname{NSOURC}(100)$,

2 RFTYPE (100), RFVAL (100), RFXLOC (100), RFYLOC (100),

3 NODERF (100), $\operatorname{RFDIST}(100), \operatorname{ICODE}(100,15), \operatorname{IPRECP}(100)$,

4 QUANTY $(100,15), \operatorname{SCXLOC}(100,15), \operatorname{SCYLOC}(100,15)$,

$5 \quad \operatorname{NODESC}(100,15), \operatorname{WANT}(100), \operatorname{NEED}(100), \operatorname{USXPLT}(100)$,

$6 \operatorname{USYPLT}(100), \operatorname{ISIDRF}(100), \operatorname{IAKNT}(100), \operatorname{ITIMFC}(100,15)$,

$7 \quad \operatorname{TIMFAC}(100,12)$

INTEGER USERID, USRTYP, RFTYPE

REAL NUMUSR, NEED

COMMON /PHYSIO/ STCOEF, TRANS, PET (12), DRINK, SESNAL (12), RMILES (100), 1

2

3

COMMON /RESVOR/ ACDMN(12), CANLEN(100), RAIN (50)

COMMON /RESVOR/ NRES, RESSTR(25), RELEAS (25), NODRES (25), RESID (25),

1

2

3

INTEGER RESID $\operatorname{EVAP}(12), \operatorname{UNIT}(5,480)$, CNVLAT , CVLONG , SNLAT1 , SNLAT2, SNUOS 1 , SNUOS2, SNUOS3, PREFAC, SEEPFC, PRSPFC, TAILFC, AGDMID (12) , CANLEN(100) RAIN(50)

REAL NACTIV

C

DO 90 IUSER $=1$, NUSERS

IF (USRTYP(IUSER) .GT. 5) GO TO 50

C

GO TO $(10,20,30,40,100)$, USRTYP(IUSER)

$\mathrm{C}$

C

10 NEED (IUSER) = PET (JMONTH) $*$ NUMUSR (IUSER)

IF (DMDFAC (IUSER) .LT. 0.) WANT (IUSER) =

1 AGDMND (JMONTH) *NUMUSR (IUSER)*(-DMDFAC (IUSER))

IF (DMDFAC (IUSER) . GE. 0.) WANT (IUSER) =

1 PET (JMONTH) *NUMUSR (IUSER) *DMDFAC (IUSER)

GO TO 90

C

$\mathrm{C}$

C

$20 \operatorname{NEED}($ IUSER $)=($ DRINK $+\operatorname{AGDMND}($ JMONTH $)) *$ NUMUSR (IUSER $)$

GO TO 80

C

$\mathrm{C}$

C

30 NEED (IUSER) $=$ DRINK $*$ NUMUSR (IUSER)

GO TO 80 
Attachment 8--FORTRAN Source Code of Subroutines for Water-Supply Operations Simulation--Continued

817

818

819

820

821

822

823

824

825

826

827

828

829

830

831

832

833

834

835

836

837

838

839
C

C

$\mathrm{C}$

40 NEED $($ IUSER $)=\operatorname{SESNAL~}($ MMONTH $) \div$ NUMUSR (IUSER) GO TO 80

C

C RESERVOIR OPERATOR

$\mathrm{C}$

50 IACCNT $=$ IAKNT (IUSER)

IF (DMDFAC (IUSER) .LT. 0.) GO TO 10

DO 60 IRES $=1$, NRES

IF (USRTYP (IUSER) .NE. RESID(IRES)) GO TO 60

NEED (IUSER) = NUMUSR (IUSER) - SBSTOR (IRES, IACCNT)

WANT (IUSER) $=$ NEED (IUSER)

GO TO 90

60 CONTINUE

C

80 WANT (IUSER) $=$ NEED (IUSER) $*$ DMDFAC (IUSER)

90 CONTINUE

C

RETURN

100 STOP

END 
Attachment 8--FORTRAN Source Code of Subroutines

for Water-Supply Operations Simulation--Continued

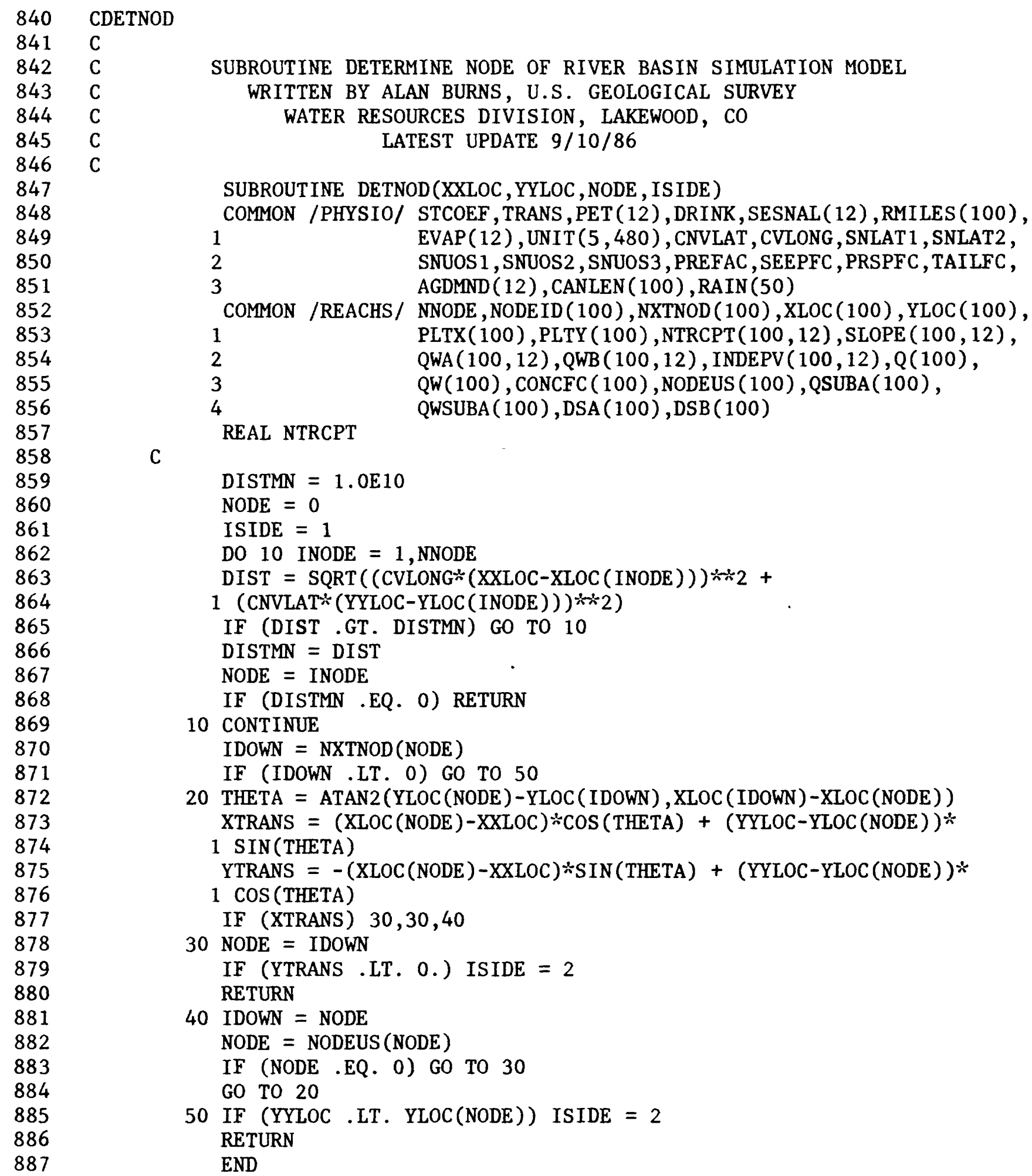


Attachment 8--FORTRAN Source Code of Subroutines

for Water-Supply Operations Simulation--Continued

888 CNSTRK2

$889 \mathrm{C}$

$890 \quad C$

$891 \quad \mathrm{C}$

$892 \mathrm{C}$

$893 \mathrm{C}$

$894 \mathrm{C}$

895

896

897

898

899

900

901

902

903

904

905

906

907

908

909

910

911

912

913

914

915

916

917

918

919

920

921

922

923

924

925

926

927

928

929

930

931

932

933

934

935

936

937

938

939

940

C

SUBROUTINE INSTRUCTION OF RIVER BASIN SIMULATION MODEL (非)

WRITTEN BY ALAN BURNS, U.S. GEOLOGICAL SURVEY

WATER RESOURCES DIVISION, LAKEWOOD, CO

LATEST UPDATE $9 / 22 / 87$

SUBROUTINE NSTRK2

COMMON NMONTH, NINDV, IN , IOUT, IPRINT , IGRAPH, IBATCH, IQW , IMONTH,

1 JMONTH, IYEAR

\section{CALL QUESZN (ITEMP)}

WRITE (IOUT, 1000)

1000 FORMAT (73H Three additional data sets must be prepared prior to $u$ 1sing the model for ,/, 22H water-use simulation: ,/,

$259 \mathrm{H}$ Additional physical descriptions of the modeled area; ,/,

$348 \mathrm{H}$ a description of the water use system; and ,/,

$441 \mathrm{H}$ a prioritized list of water rights. ,/,

$560 \mathrm{H}$ The format for the additional physical description file is:

$6, /$,

7 37H Record Columns Name Description ,/,

$880 \mathrm{H}+$

$9+-1$,

$* 33 \mathrm{H} \quad 1 \quad 1-80$ TITLEM

$176 \mathrm{H} 229-14$ PET

2 , in feet, one ,/,

$378 \mathrm{H} \quad 15-20$ PET

4nning in January ,/,

$521 \mathrm{H} \quad 21-26$

$621 \mathrm{H} \quad 33-38$

$721 \mathrm{H}$

$45-50$

$821 \mathrm{H}$

$57-62$

$921 \mathrm{H}$

69- 74

WRITE (IOUT, 1010)

1010 FORMAT $(76 \mathrm{H} 3$

1 feet, one for each of ,/,

$266 \mathrm{H} \quad 15-20$ EVAP

a title ,/,

3uary ,/,

PET ,/, $21 \mathrm{H}$

PET,$/, 21 \mathrm{H}$

PET,$/, 21 \mathrm{H}$

PET,$/, 21 \mathrm{H}$

PET ,/, 21H

monthly potential evapotranspiration

$422 \mathrm{H}$

$522 \mathrm{H}$

$21-26$

$33-38$

$45-50$

$57-62$

$69-74$

$722 \mathrm{H}$

$9-14$

$978 \mathrm{H}$

4

*nd, in feet, one ,/,

$178 \mathrm{H}$

$15-20$

2nning in January ,/,

$324 \mathrm{H} 21-26$

$424 \mathrm{H}$

$33-38$

$524 \mathrm{H}$

$45-50$

$624 \mathrm{H}$

$57-62$

$724 \mathrm{H}$

$69-74$

EVAP , /, $22 \mathrm{H}$

EVAP ,/, $22 \mathrm{H}$

EVAP,$/, 22 \mathrm{H}$

EVAP,$/, 22 \mathrm{H}$

EVAP,$/, 22 \mathrm{H} \quad 75-80$ EVAP ,/,

for each of the 12 months, begi

AGDMND monthly agricultural irrigation dema

WRITE (IOUT, 1020)

AGDMND

AGDMND , $/, 24 \mathrm{H}$

AGDMND , /, 24H

AGDMND ,/, 24H

AGDMND , /, 24H

AGDMND , /, 24H

$\begin{array}{ll}27-32 & \text { PET ,/, } \\ 39-44 & \text { PET ,/, } \\ 51-56 & \text { PET ,/, } \\ 63-68 & \text { PET ,/, } \\ 75-80 & \text { PET ) }\end{array}$

monthly lake evaporation, in

the 12 months, beginning in Jan

for each of the 12 months, begi

$$
\begin{aligned}
& \text { 27- } 32 \text { EVAP ,/, } \\
& 39-44 \text { EVAP }, /, \\
& 51-56 \text { EVAP ,/, } \\
& 63-68 \text { EVAP ,/, } \\
& 75-80 \text { EVAP ,/, } \\
& \text { ultural irrigation dema } \\
& \text { of the } 12 \text { months, beg } \\
& 27-32 \text { AGDMND ,/, } \\
& 39-44 \text { AGDMND ,/, } \\
& 51-56 \text { AGDMND ,/, } \\
& 63-68 \text { AGDMND ,/, } \\
& 75-80 \text { AGDMND ) }
\end{aligned}
$$


Attachment 8--FORTRAN Source Code of Subroutines for Water-Supply Operations Simulation--Continued

941

942

943

944

945

946

947

948

949

950

951

952

953

954

955

956

957

958

959

960

961

962

963

964

965

966

967

968

969

970

971

972

973

974

975

976

977

978

979

980

1020 FORMAT ( $75 \mathrm{H} 59-14$ SESNAL monthly instream demand, in $\mathrm{f}$ leet, one for each of ,/,

$266 \mathrm{H}$

15- 20 SESNAL

the 12 months, beginning in Jan

3uary,$/$,

$424 \mathrm{H}$

$524 \mathrm{H}$

$624 \mathrm{H}$

$724 \mathrm{H}$

$824 \mathrm{H}$

$979 \mathrm{H}$

21- 26 SESNAL ,/, 24H

33- 38 SESNAL , $/, 24 \mathrm{H}$

45- 50 SESNAL , $/, 24 \mathrm{H}$

57- 62 SESNAL ,/, 24H

69- 74 SESNAL ,/, 24H

27- 32 SESNAL ,/,

39- 44 SESNAL ,/,

51- 56 SESNAL ,/,

63- 68 SESNAL ,/,

75- 80 SESNAL ,/,

*t (dimensionless) ,/,

$175 \mathrm{H}$

2 feet squared ,/,

3 31X, 7Hper day ,/,

$479 \mathrm{H} 79-14$ DRINK average municipal water demand, in a 5 cre-feet per unit ,/,

$674 \mathrm{H} \quad 8$ 9- 14 CNVLAT factor to convert latitude, in degre 7 es, to miles ,/,

$875 \mathrm{H} 15-20$ CVLONG factor to convert longitude, in degr 9 ees, to miles)

WRITE (IOUT, 1030)

1030 FORMAT (80H

liver miles east of SNLAT1,/,

$280 \mathrm{H} 27-32$ SNUOS2 sinuosity factor to compute river mi 31es between SNLAT1 ,/,

$431 \mathrm{X}, 10 \mathrm{Hand}$ SNLAT2,$/$,

$580 \mathrm{H}$

33- 38 SNUOS3 sinuosity factor to compute river mi 6les west of SNLAT2 ,/,

$762 \mathrm{H}$

39- 44 SNLAT1 latitude divider, in decimal degrees

$8, /$,

$962 \mathrm{H}$

$*, /$,

$173 \mathrm{H}$

45- 50 SNLAT2 latitude divider, in decimal degrees

2lization of ,/,

$331 \mathrm{X}, 12$ Hreturn flows, /,

$469 \mathrm{H}$

5er mile, $/$,

$675 \mathrm{H}$ 63- 68 PRSPFC limit of effective monthly precipita 7 tion, in feet)

WRITE (IOUT, 1040) 
Attachment 8--FORTRAN Source Code of Subroutines

for Water-Supply Operations Simulation--Continued

1011

1012

1013

1014

1015

1016

1017

1018

1019

1020

1021

1022

1023
1040 FORMAT (72H

1 goes through the ,/,

$231 \mathrm{X}, 44 \mathrm{Hg}$ round-water system (ranges from 0.0 to 1.0 ) ,/,

$366 \mathrm{H} 91-5 \mathrm{NRES}$ the number of reservoirs (maximum of 4 25),/,

$557 \mathrm{H}$ note -- record 10 is entered as a set, one for each NRES ,/,

$647 \mathrm{H} 10 \quad 1-5$ RESID a reservoir ID number ,/,

$742 \mathrm{H} 6-13$ RESNAM a reservoir name ,/,

$869 \mathrm{H} \quad 16-25$ CAPMAX maximum capacity of reservoir, in ac

9 re-feet ,/,

$* 70 \mathrm{H} 26-35$ NACTIV inactive capacity of reservoir, in a

1cre-feet ,/,

$269 \mathrm{H} 36-45$ AREAMX surface area, in acres, at maximum c

3apacity ,/,

$455 \mathrm{H}$

$554 \mathrm{H}$

46- 55 RSXLOC longitude, in decimal degrees, /, 56- 65 RSYLOC latitude, in decimal degrees, $/$,

$631 \mathrm{X}, 49 \mathrm{HF}$ or on-channel reservoirs, RSXLOC and RSYLOC must ,/,

$731 \mathrm{X}, 49 \mathrm{Hcorrespond}$ exactly to the XLOC and YLOC of a node ,/,

$866 \mathrm{H} \quad 66-75$ RESSTR initial reservoir contents, in acre9 feet )

WRITE (IOUT, 1050)

1050 FORMAT $(76 \mathrm{H}$

76- 85 RSCONC initial water-quality concent

1ration, in milligrams,/,

$231 \mathrm{X}, 9$ Hper liter ,/,

$378 \mathrm{H} \quad 86-90$

4 ymbol, in inches, $/$,

$578 \mathrm{H} 91-95$ RSYPLT plotting offset from the reservoir $\mathrm{s}$ 6ymbol, in inches ,/,

$758 \mathrm{H}$ note -- record 11 is entered as a set, one for each NNODE ,/,

$876 \mathrm{H} 11 \quad 9-14$ GWSTOR initial ground-water storage above $\mathrm{n}$ 9ode on side $1,, /$,

* $31 \mathrm{X}, 12 \mathrm{Hin}$ acre-feet,$/$,

$175 \mathrm{H} \quad 15-20$ GWCONC initial water-quality concentration

2above node on ,/,

$331 \mathrm{X}, 31 \mathrm{Hside} 1$, in milligrams per liter ,/,

$476 \mathrm{H} 21-26$ GWSTOR initial ground-water storage above $\mathrm{n}$

5ode on side $2,, /$,

$631 \mathrm{X}, 12 \mathrm{Hin}$ acre-feet ,/,

$775 \mathrm{H} \quad 27-32$ GWCONC initial water-quality concentration

8above node on ,/,

$931 \mathrm{X}, 31 \mathrm{Hs}$ ide 2, in milligrams per liter )

CALL QUESZN (ITEMP)

WRITE (IOUT, 1060) 
Attachment 8--FORTRAN Source Code of Subroutines

for Water-Supply Operations Simulation--Continued

1024

1025

1026

1027

1028

1029

1030

1031

1032

1033

1034

1035

1036

1037

1038

1039

1040

1041

1042

1043

1044

1045

1046

1047

1048

1049

1050

1051

1052

1053

1054

1055

1056

1057

1058

1059

1060

1061

1062

1063

1064

1065

1066

1067

1068

1069

1070

1071

1072

1060 FORMAT (38H The format for the water use file is: ,/,

$137 \mathrm{H}$ Record Columns Name Description ,/,

$280 \mathrm{H}+$

3

4 33H 1 1- 80 TITLEU a title ,/,

$568 \mathrm{H} 21-5$ NUSERS the number of water users (maximum o 6f 100), /,

$773 \mathrm{H}$ note -- records $3,4,5$, and 6 are entered as a set, one for 8each NUSERS ,/,

$948 \mathrm{H} 3$ 1- 5 USERID a water user ID number ,/,

* 44H 6- 13 USRNAM a water user name ,/,

$149 \mathrm{H} 16-20$ USRTYP the type of water user ,/,

$231 \mathrm{X}, 16 \mathrm{H} 1$ = agricultural ,/,

$331 \mathrm{X}, 13 \mathrm{H} 2$ = municipal ,/,

$431 \mathrm{X}, 14 \mathrm{H} 3=$ industria1,$/$,

$531 \mathrm{X}, 13 \mathrm{H} 4=$ in stream,$/$,

$631 \mathrm{X}, 22 \mathrm{H} 5=$ reservoir operator ,/,

$763 \mathrm{H} \quad 21-30$ NUMUSR the quantity served by the water use $8 \mathrm{r}$ )

WRITE (IOUT, 1070)

1070 FORMAT $(31 \mathrm{X}, 45 \mathrm{Hif}$ type 1 , then the units are irrigated acres,$/$,

$131 \mathrm{X}, 44 \mathrm{Hif}$ type 2 , then the units are populace units ,/,

$231 X, 44 \mathrm{Hif}$ type 3 , then the units are units produced ,/,

$331 \mathrm{X}, 42 \mathrm{Hif}$ type 4 , then the units are actual water ,/,

$436 \mathrm{X}, 20$ Hneeded, in acre-feet ,/,

$531 \mathrm{X}, 49 \mathrm{Hif}$ type 5 , then the units are reservoir capacity ,/,

$636 \mathrm{X}, 35$ Havailable to the user, in acre-feet, /,

$775 \mathrm{H} \quad 31-40$ DMDFAC factor to compute a water user's dem 8 and for water ,/,

$931 \mathrm{X}, 38 \mathrm{Hif}$ value is positive, multiply by PET, ,/,

* $31 \mathrm{X}, 41 \mathrm{Hif}$ value is negative, multiply by AGDMND. ,/,

$155 \mathrm{H} \quad 41-50$ XLOCU longitude, in decimal degrees ,/,

$254 \mathrm{H} \quad 51-60$ YLOCU latitude, in decimal degrees ,/,

$331 \mathrm{X}, 45 \mathrm{HF}$ or reservoir operators, XLOCU and YLOCU must ,/,

$436 \mathrm{X}, 44 \mathrm{Hcorrespond}$ exactly to the RSXLOC and RSYLOC ,/,

$536 \mathrm{X}, 14 \mathrm{Hof}$ a reservoir ,/,

$679 \mathrm{H} 61-65$ USXPLT plotting offset from the water user

7 symbol, in inches ,/,

$879 \mathrm{H} \quad 66-70$ USYPLT plotting offset from the water user

9 symbol, in inches)

WRITE (IOUT, 1080)

1080 FORMAT (78H

71- 75 IPRECP number indicating which of th

le independent variables ,/,

$231 \mathrm{X}, 49 \mathrm{Hrepresents} \mathrm{the} \mathrm{appropriate} \mathrm{precipitation} \mathrm{data} \mathrm{for} \mathrm{,/,}$

$331 \mathrm{X}, 15 \mathrm{Hthe}$ water user. ,/,

$473 \mathrm{H} \quad 76-80$ IAKNT reservoir subaccout number appropria

5 te for this ,/,

$631 \mathrm{X}, 10 \mathrm{Hwater}$ user )

WRITE (IOUT, 1090) 
Attachment 8--FORTRAN Source Code of Subroutines for Water-Supply Operations Simulation--Continued

1073

1074

1075

1076

1077

1078

1079

1080

1081

1082

1083

1084

1085

1086

1087

1088

1089

1090

1091

1092

1093

1094

1095

1096

1097

1098

1099

1100

1101

1102

1103

1104

1105

1106

1107

1108

1109

1110

1090 FORMAT $(69 \mathrm{H} 4 \quad 46-20$ NSOURC number of sources available $t$

40 a water user, $/$,

$531 \mathrm{X}, 15 \mathrm{H}$ (maximum of 15),/,

$645 \mathrm{H} 21-25$ RFTYPE type of return flow ,/,

$731 \mathrm{X}, 45 \mathrm{H} 1$ - a percentage of initially computed return ,/,

$836 \mathrm{X}, 44 \mathrm{Hfl}$ low becomes deep perocolation (rest is tail ,/,

$936 \mathrm{X}, 6 \mathrm{Hwater}), /$,

* $31 \mathrm{X}, 45 \mathrm{H} 2$ - a percentage of total application becomes ,/,

$136 \mathrm{X}, 36 \mathrm{Htail}$ water (rest is lost from basin) ,/,

$231 \mathrm{X}, 45 \mathrm{H} 3$ - a percentage of total application becomes ,/,

$336 \mathrm{X}, 42 \mathrm{Hdeep}$ percolation (rest is lost from basin) ,/,

$431 \mathrm{X}, 45 \mathrm{H} 4$ - a percentage of initially computed return ,/,

$536 \mathrm{X}, 42 \mathrm{Hflow}$ becomes tail water (rest is lost from ,/,

$636 \mathrm{X}, 6 \mathrm{Hbasin}$ ) ,/,

$731 \mathrm{X}, 45 \mathrm{H} 5$ - a percentage of initially computed return ,/,

$836 \mathrm{X}, 43 \mathrm{Hfl}$ low becomes deep percolation (rest is lost ,/,

$936 \mathrm{X}, 11 \mathrm{Hfrom}$ basin) )

WRITE (IOUT, 1100)

1100 FORMAT $(48 \mathrm{H}$

$155 \mathrm{H}$

$254 \mathrm{H}$

$36-45$

26- 35 RFVAL return flow percentage,/,

46- 55 RFYLOC latitude, in decimal degrees,

$376 \mathrm{H} \quad 56-65$ RFDST distance from point of deep percolat

4ion to stream, ,/,

$531 \mathrm{X}, 48 \mathrm{Hin}$ feet. (if 0 ., the program computes this value ,/,

$636 \mathrm{X}, 15$ Husing location) ,/,

$742 \mathrm{H}$ note - record 5 is repeated NSOURC times ,/,

$846 \mathrm{H} 5$ 1- 5 ICODE type of water source,/,

$931 \mathrm{X}, 20 \mathrm{H} 1=$ direct diversion , $/$,

$* 31 \mathrm{X}, 24 \mathrm{H} 2$ = ground-water pumpage,$/$,

$131 \mathrm{X}, 44 \mathrm{H} 3$ = reservoir release (if reservoir releases ,/,

$236 \mathrm{X}, 44 \mathrm{Hdi}$ rectly to the water user rather than using ,/,

$336 \mathrm{X}, 33 \mathrm{H}$ the river as a conduit, enter -3) ,/,

$431 \mathrm{X}, 41 \mathrm{H} 4=$ direct pipeline or tunnel importation ,/,

$531 \mathrm{X}, 21 \mathrm{H} 5=$ storage diversion $/$,

$631 \mathrm{X}, 47 \mathrm{H} 6$ = direct diversion used for reservoir storage ,/,

$764 \mathrm{H}$

6- 15 QUANTY

$8 \mathrm{ce}$ )

WRITE (IOUT, 1110) 
Attachment 8--FORTRAN Source Code of Subroutines

for Water-Supply Operations Simulation--Continued

1111

1112

1113

1114

1115

1116

1117

1118

1119

1120

1121

1122

1123

1124

1125

1126

1127

1128

1129

1130

1131

1132

1133

1134

1135

1136

1137

1138

1139

1140

1141

1142

1143

1144

1145

1146

1147

1148

1149

1150

1151

1152

1153

1154

1155

1156

1157

1158

1159

1160

1110 FORMAT $(31 \mathrm{X}, 46 \mathrm{Hif}$ type 1 , then the legal right, in cubic feet ,/,

$136 \mathrm{X}, 10$ Hper second ,/,

$231 \mathrm{X}, 46 \mathrm{Hif}$ type 2 , then the pumping capacity, in cubic ,/,

$336 \mathrm{X}, 15 \mathrm{Hf}$ eet per second,/,

$431 \mathrm{X}, 45 \mathrm{Hif}$ type 3 , then the capacity of the reservoir ,/,

$536 \mathrm{X}, 42 \mathrm{Hallocated}$ to this water user, in acre-feet ,/,

$636 \mathrm{X}, 44 \mathrm{H}$ (if the reservoir is a 'general-use', 'bank- ,/,

$736 \mathrm{X}, 44 \mathrm{Haccount}$ ' type storage, enter the percentage ,/,

$836 \mathrm{X}, 37 \mathrm{Hallocated}$ to this user as a negative),/,

$931 \mathrm{X}, 47 \mathrm{Hif}$ type 4 , then the pipeline or tunnel carrying ,/,

* 36X,22Hcapacity, in acre-feet ,/,

$131 \mathrm{X}, 45 \mathrm{Hif}$ type 5 , then the legal right, in acre-feet, $/$,

$231 \mathrm{x}, 46 \mathrm{Hif}$ type 6 , then the legal right, in cubic feet ,/,

$336 \mathrm{X}, 10 \mathrm{Hper}$ second ,/,

$455 \mathrm{H} \quad 16-25$ SCXLOC longitude, in decimal degrees, /,

$554 \mathrm{H} \quad 26-35$ SCYLOC latitude, in decimal degrees , $/$,

$680 \mathrm{H} \quad 36-45$ GWDST the distance from point of pumpage $t$

70 stream, in feet. ,/,

$831 \mathrm{X}, 44 \mathrm{HIf} 0$. , the program computes this value using ,/,

$931 \mathrm{X}, 9 \mathrm{Hl}$ location. )

WRITE (IOUT, 1120)

1120 FORMAT (76H 46- 50 NMRES the ID number of the appropri 1ate reservoir (RESID) ,/,

$278 \mathrm{H} \quad 51-55$ IACCNT subaccount number to identify portio $3 \mathrm{n}$ of a reservoir ,/,

$431 \mathrm{X}, 45 \mathrm{Ha}$ llocated to this water user (if 0 , set to 1 ), /,

$574 \mathrm{H} \quad 56-60$ ITIMFC code to indicate whether the source

6 is available, /,

$731 \mathrm{X}, 44 \mathrm{Hevery}$ month (if 0 , it is; if 1 , read TIMFAC), $/$,

$853 \mathrm{H}$ note - record 6 is read only when ITIMFC is nonzero ,/,

$973 \mathrm{H} 66-10$ TIMFAC percentage indicating how much of th

*e source is ,/,

$173 \mathrm{H} \quad 11-15$ TIMFAC

2 , beginning ,/,

$373 \mathrm{H} \quad 16-20$ TIMFAC

4 cates fully, /,

$576 \mathrm{H} \quad 21-25$ TIMFAC

6 the source is ,/,

$769 \mathrm{H} 26-30$ TIMFAC inactive during that particular

8 month. )

WRITE (IOUT, 1130)

1130 FORMAT $(24 \mathrm{H}$

$124 \mathrm{H}$

$224 \mathrm{H}$

$324 \mathrm{H}$

$424 \mathrm{H}$

$524 \mathrm{H}$

$624 \mathrm{H}$

CALL QUESZN (ITEMP)

36- 40 TIMFAC, , ,

41- 45 TIMFAC ,/,

46- 50 TIMFAC ,/,

51- 55 TIMFAC ,/,

WRITE (IOUT, 1140)

active in each of the 12 months

in January. A value of 1 . indi

active, a value of 0 . indicates

WRITE (IOUT, 1140) 
Attachment 8--FORTRAN Source Code of Subroutines

for Water-Supply Operations Simulation--Continued

1161

1162

1163

1164

1165

1166

1167

1168

1169

1170

1171

1172

1173

1174

1175

1176

1177

1178

1179

1180
1140 FORMAT ( $41 \mathrm{H}$ The format for the water rights file is: ,/,

$137 \mathrm{H}$ Record Columns Name Description,/,

$280 \mathrm{H}+$

$3+, 1$,

$4 \longdiv { 3 3 \mathrm { H } } 1 \mathrm { 1 } - 8 0$ TITLER a title ,/,

$569 \mathrm{H} 21-5$ NRIGHT the number of water rights (maximum 6of 200),/,

$742 \mathrm{H}$ note -- record 3 is repeated NRIGHT times ,/,

$871 \mathrm{H} 31-5$ OWNER the water user ID (USERID) who owns

9 the right, /,

$* 73 \mathrm{H}$ 6- 13 NAME the water user name (USRNAM) who own

1s the right, /,

$254 \mathrm{H} \quad 16-23$ DATE

$379 \mathrm{H} \quad 24-33$ AMNT

the date of the water right, /,

the amount of the water right (QUANT

$4 \mathrm{Y})$, in cubic feet ,/,

$531 \mathrm{X}, 40 \mathrm{Hper}$ second for direct diversions, and in ,/,

$631 \mathrm{X}, 33 \mathrm{Hacre}-\mathrm{feet}$ for storage diversions. ,/,

$755 \mathrm{H}$ note - all of the record 3 's must be ordered by DATE. ) RETURN

END 
Attachment 8--FORTRAN Source Code of Subroutines

for Water-Supply Operations Simulation--Continued

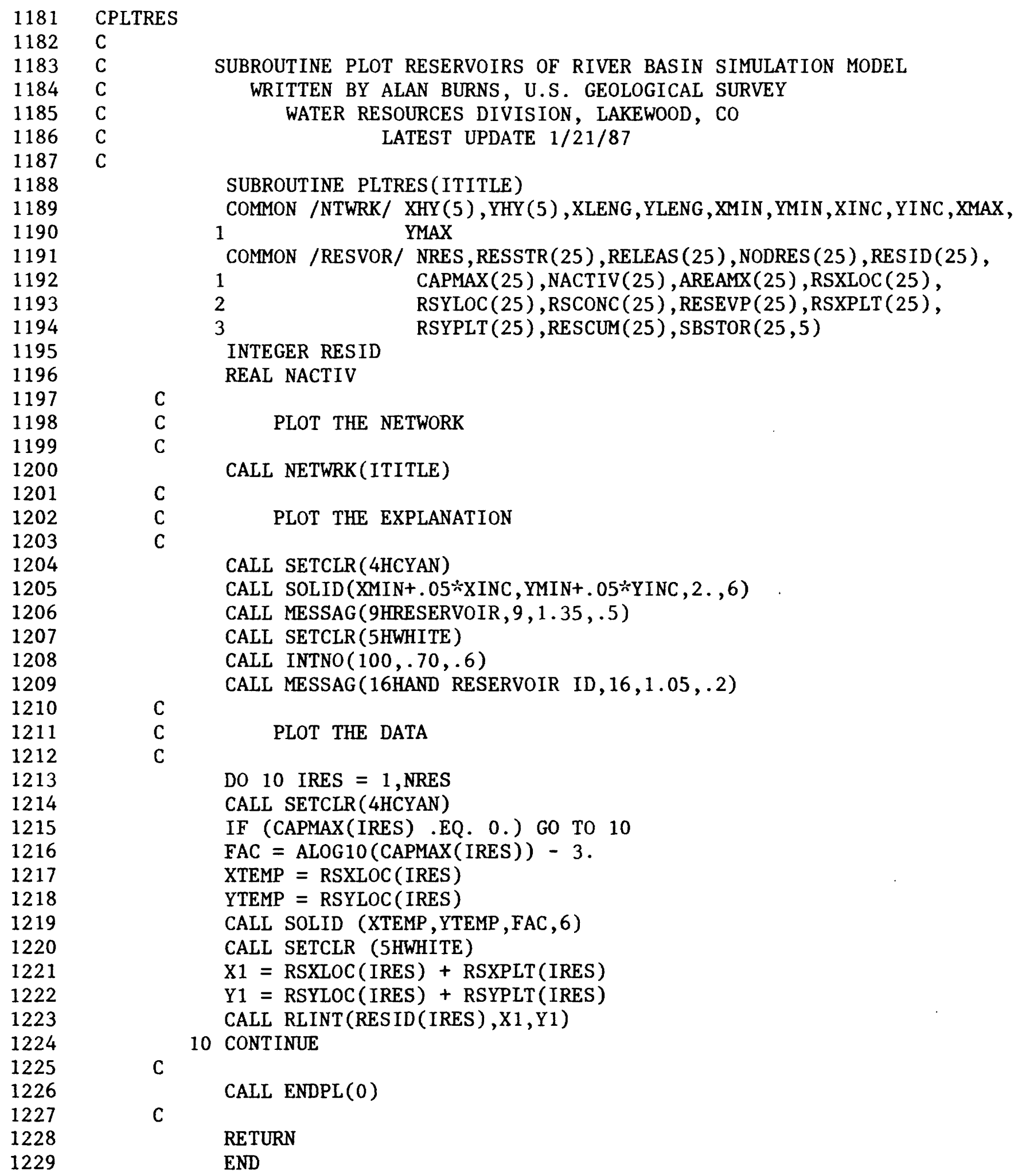


Attachment 8--FORTRAN Source Code of Subroutines

for Water-Supply Operations Simulation--Continued

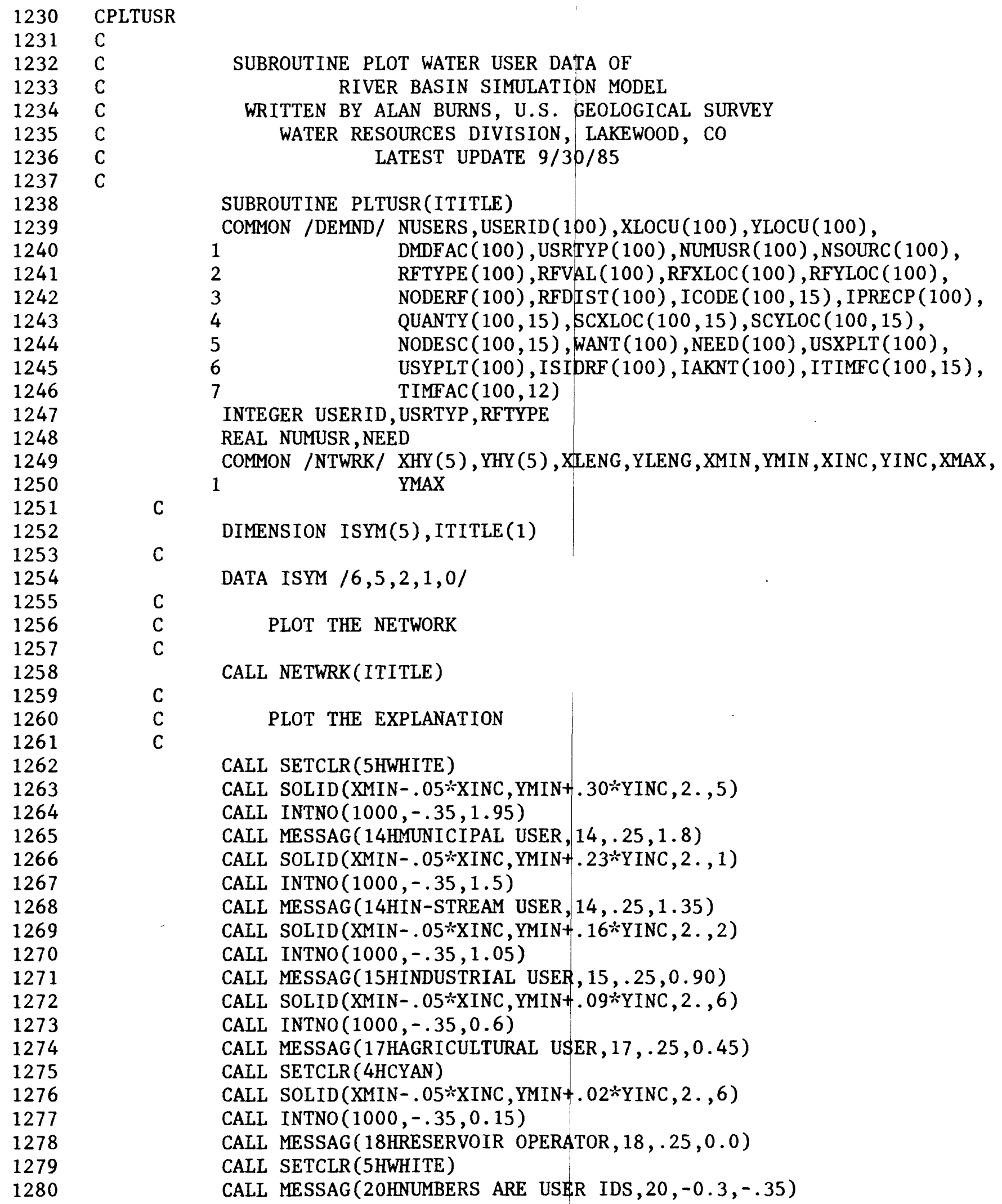


Attachment 8--FORTRAN Source Code of Subroutines

for Water-Supply Operations Simulation--Continued

1281

1282

1283

1284

1285

1286

1287

1288

1289

1290

1291

1292

1293

1294

1295

1296

1297

1298

1299

1300

1301

1302

1303

1304

1305

1306

1307

1308

1309

1310

1311

1312

1313

1314

1315

1316

1317

1318

1319

1320

1321

1322

1323

1324

1325

1326

1327

1328

1329

1330

1331

1332

1333

1334

1335

1336
C

CALL MESSAG(25HSOURCES OF WATER INCLUDE : ,25,7.2,1.5)

CALL SETCLR (4HCYAN)

CALL MESSAG (17HDIRECT DIVERSIONS $17,7.8,1.2)$

CALL SETCLR (3HRED)

CALL MESSAG (20HGROUND-WATER PUMPAGE , 20,7.8,0.9)

CALL SETCLR (5HGREEN)

CALL MESSAG (18HRESERVOIR RELEASES , 18, 7.8,0.6)

CALL SETCLR (7HMAGENTA)

CALL MESSAG(7HIMPORTS $, 7,7.8,0.3$ )

CALL SETCLR (5HWHITE)

CALL MESSAG (14HALSO SHOWN IS : $14,7.2,0$. )

CALL MESSAG(11HRETURN FLOW, $11,7.8,-0.3)$

C

C

C

PLOT THE DATA

DO 110 IUSER $=1$, NUSERS

CALL SETCLR(5HWHITE)

IPEN = USRTYP (IUSER)

IF (NUMUSR(IUSER) .EQ. 0.) GO TO 110

$F A C=($ ALOG10 $($ NUMUSR $($ IUSER $))-1) /$.

IF (IPEN .LT. 5) GO TO 10

IPEN $=1$

CALL SETCLR(4HCYAN)

$\mathrm{FAC}=1.5$

10 XTEMP $=$ XLOCU (IUSER)

YTEMP $=$ YLOCU (IUSER)

CALL SOLID (XTEMP, YTEMP, FAC, ISYM(IPEN))

$\mathrm{X} 1=\mathrm{XLOCU}($ IUSER$)+\mathrm{USXPLT}$ (IUSER)

$Y 1=$ YLOCU (IUSER) + USYPLT (IUSER)

20 CALL RLINT (USERID (IUSER), X1,Y1)

CALI SETCLR(5HWHITE)

IF (RFXLOC (IUSER) .EQ. 0. .OR. RFYLOC (IUSER) .EQ. 0.) GO TO 30

CALL RLVEC (XIOCU(IUSER), YLOCU(IUSER), RFXLOC (IUSER), RFYLOC (IUSER), $10)$

30 NSORC $=$ NSOURC (IUSER)

DO 100 ISOURC $=1$, NSORC

IPEN = ICODE (IUSER, ISOURC)

GO TO $(40,50,60,70,80)$, IPEN

40 CALL SETCLR (4HCYAN)

GO TO 90

50 CALL SETCLR(3HRED)

GO TO 90

60 CALL SETCLR (5HGREEN)

GO TO 90

70 CALL SETCLR (7HMAGENTA)

GO TO 90

80 CALL SETCLR (4HCYAN)

90 CALL RLVEC (XLOCU(IUSER), YLOCU(IUSER), SCXLOC (IUSER, ISOURC),

1 SCYLOC (IUSER, ISOURC), 0)

100 CONTINUE

110 CONTINUE

CALL ENDPL(0)

C

RETURN

END 
Attachment 8--FORTRAN Source Code of Subroutines

for Water-Supply Operations Simulation--Continued

\begin{tabular}{|c|c|c|}
\hline $\begin{array}{l}1337 \\
1338\end{array}$ & $\begin{array}{l}\text { CPRSTRS } \\
\mathrm{C}\end{array}$ & \\
\hline 1339 & $\mathrm{C}$ & SUBROUTINE PRESTRESS OF RIVER BASIN SIMULATION MODEL \\
\hline 1340 & $\mathrm{C}$ & WRITTEN BY ALAN BURNS, U.S. GEOLOGICAL SURVEY \\
\hline 1341 & $\mathrm{C}$ & WATER RESOURCES DIVISION, LAKEWOOD, CO \\
\hline 1342 & C & LATEST UPDATE $4 / 28 / 87$ \\
\hline 1343 & $\mathrm{C}$ & \\
\hline 1344 & & SUBROUTINE PRSTRS \\
\hline 1345 & & COMMON NMONTH, NINDV, IN , IOUT , IPRINT , IGRAPH, IBATCH, IQW, IMONTH, \\
\hline 1346 & & JMONTH, IYEAR \\
\hline 1347 & & COMMON /DEMND/ NUSERS,USERID(100),XLOCU (100), YLOCU(100), \\
\hline 1348 & & DMDFAC (100), USRTYP (100), NUMUSR (100), NSOURC (100), \\
\hline 1349 & & $\operatorname{RFTYPE}(100), \operatorname{RFVAL}(100), \operatorname{RFXLOC}(100), \operatorname{RFYLOC}(100)$, \\
\hline 1350 & & $\operatorname{NODERF}(100), \operatorname{RFDIST}(100), \operatorname{ICODE}(100,15), \operatorname{IPRECP}(100)$ \\
\hline 1351 & & $\operatorname{QUANTY}(100,15), \operatorname{SCXLOC}(100,15), \operatorname{SCYLOC}(100,15)$, \\
\hline 1352 & & NODESC $(100,15), \operatorname{WANT}(100), \operatorname{NEED}(100), \operatorname{USXPLT}(100)$, \\
\hline 1353 & & USYPLT $(100), \operatorname{ISIDRF}(100), \operatorname{IAKNT}(100), \operatorname{ITIMFC}(100,15)$ \\
\hline 1354 & & $\operatorname{TIMFAC}(100,12)$ \\
\hline 1355 & & INTEGER USERID, USRTYP, RFTYPE \\
\hline 1356 & & REAL NUMUSR, NEED \\
\hline 1357 & & COMMON /PHYSIO/ STCOEF, TRANS, PET (12), DRINK, SESNAL(12), RMILES (100), \\
\hline 1358 & & $1 \quad \operatorname{EVAP}(12), \mathrm{UNIT}(5,480)$, CNVLAT , CVLONG, SNLAT 1, SNLAT2, \\
\hline 1359 & & SNUOS 1 , SNUOS 2 , SNUOS 3 , PREFAC, SEE PFC, PRSPFC, TAILFC, \\
\hline 1360 & & $\operatorname{AGDMND}(12)$, CANLEN $(100), \operatorname{RAIN}(50)$ \\
\hline 1361 & & COMMON /REACHS/ NNODE, NODEID (100), NXTNOD (100), XLOC $(100), \operatorname{YLOC}(100)$, \\
\hline 1362 & & $1 \quad \operatorname{PLTX}(100), \operatorname{PLTY}(100), \operatorname{NTRCPT}(100,12), \operatorname{SLOPE}(100,12)$, \\
\hline 1363 & & $\operatorname{QWA}(100,12), \mathrm{QWB}(100,12), \operatorname{INDEPV}(100,12), \mathrm{Q}(100)$ \\
\hline 1364 & & QW $(100), \operatorname{CONCFC}(100), \operatorname{NODEUS}(100), \mathrm{QSUBA}(100)$ \\
\hline 1365 & & QWSUBA $(100), \mathrm{DSA}(100), \mathrm{DSB}(100)$ \\
\hline 1366 & & REAL NTRCPT \\
\hline 1367 & & COMMON /RESPON/ FRSPNZ $(100,480,2), \operatorname{TLWATR}(100,2), \operatorname{TWCONC}(100,2)$ \\
\hline 1368 & C & \\
\hline 1369 & C & RUN PRESTRESS FOR 10 YEARS \\
\hline 1370 & $\mathrm{C}$ & \\
\hline 1371 & & DO 130 IYEAR $=1,10$ \\
\hline 1372 & & DO 130 IMONTH $=1,12$ \\
\hline 1373 & C & \\
\hline 1374 & $\mathrm{C}$ & MOVE THE RESPONSES UP A MONTH IN TIME \\
\hline 1375 & $\mathrm{C}$ & \\
\hline 1376 & & DO 10 INODE $=1$, NNODE \\
\hline 1377 & & DO 10 ITIME $=2,480$ \\
\hline 1378 & & DO 10 ISIDE $=1,2$ \\
\hline 1379 & & FRSPNZ (INODE , ITIME- 1, ISIDE) $=$ FRSPNZ (INODE , ITIME , ISIDE) \\
\hline 1380 & & 10 CONTINUE \\
\hline 1381 & $\mathrm{C}$ & \\
\hline 1382 & & DO 20 INODE $=1$, NNODE \\
\hline 1383 & & DO 20 ISIDE $=1,2$ \\
\hline 1384 & & 20 TLWATR (INODE, ISIDE) $=0$. \\
\hline 1385 & $\mathrm{C}$ & \\
\hline 1386 & $\mathrm{C}$ & APPROXIMATE BY USING EACH USERS DEMAND \\
\hline
\end{tabular}


Attachment 8--FORTRAN Source Code of Subroutines for Water-Supply Operations Simulation--Continued

1387

1388

1389

1390

1391

1392

1393

1394

1395

1396

1397

1398

1399

1400

1401

1402

1403

1404

1405

1406

1407

1408

1409

1410

1411

1412

1413

1414

1415

1416

1417
$\mathrm{C}$

DO 130 IUSER $=1$, NUSERS

IF (USRTYP(IUSER) .GT. 5) GO TO 130

GO TO $(30,40,50,130,130)$, USRTYP(IUSER)

30 USE $=$ PET (IMONTH) *NUMUSR (IUSER)

GO TO 60

40 USE $=($ DRINK + PET $($ IMONTH $)) * N U M U S R($ IUSER $)$

GO TO 60

50 USE $=$ DRINK $*$ NUMUSR (IUSER)

60 RTRN = USE $*$ PREFAC

GO TO $(90,80,80,70,70)$, RFTYPE (IUSER)

70 RTRN = RTRN*RFVAL (IUSER)

GO TO 90

80 RTRN = USE $*$ RFVAL (IUSER)

90 IF (RTRN .LE. O.) GO TO 130

TAILWR $=0$.

GO TO $(100,110,120,110,120)$, RFTYPE (IUSER)

100 TAILWR $=\operatorname{RTRN} \div(1 .-\operatorname{RFVAL}($ IUSER $))$

GO TO 120

110 TAILWR = RTRN

120 DPPERC $=$ RTRN - TAILWR

INODE = NODERF (IUSER)

ISIDE = ISIDRF (IUSER)

TLWATR (INODE, ISIDE) = TLWATR (INODE, ISIDE) + TAILWR $*$ TAILFC

FRSPNZ (INODE , 1, ISIDE) $=$ FRSPNZ (INODE, 1, ISIDE) +

1 TAILWR $*(1$. -TAILFC $)$

CALL PRECUR(DPPERC, INODE, ISIDE, RFDIST (IUSER))

C

130 CONTINUE

RETURN

END 
Attachment 8--FORTRAN Source Code of Subroutines

\section{for Water-Supply Operations Simulation--Continued}

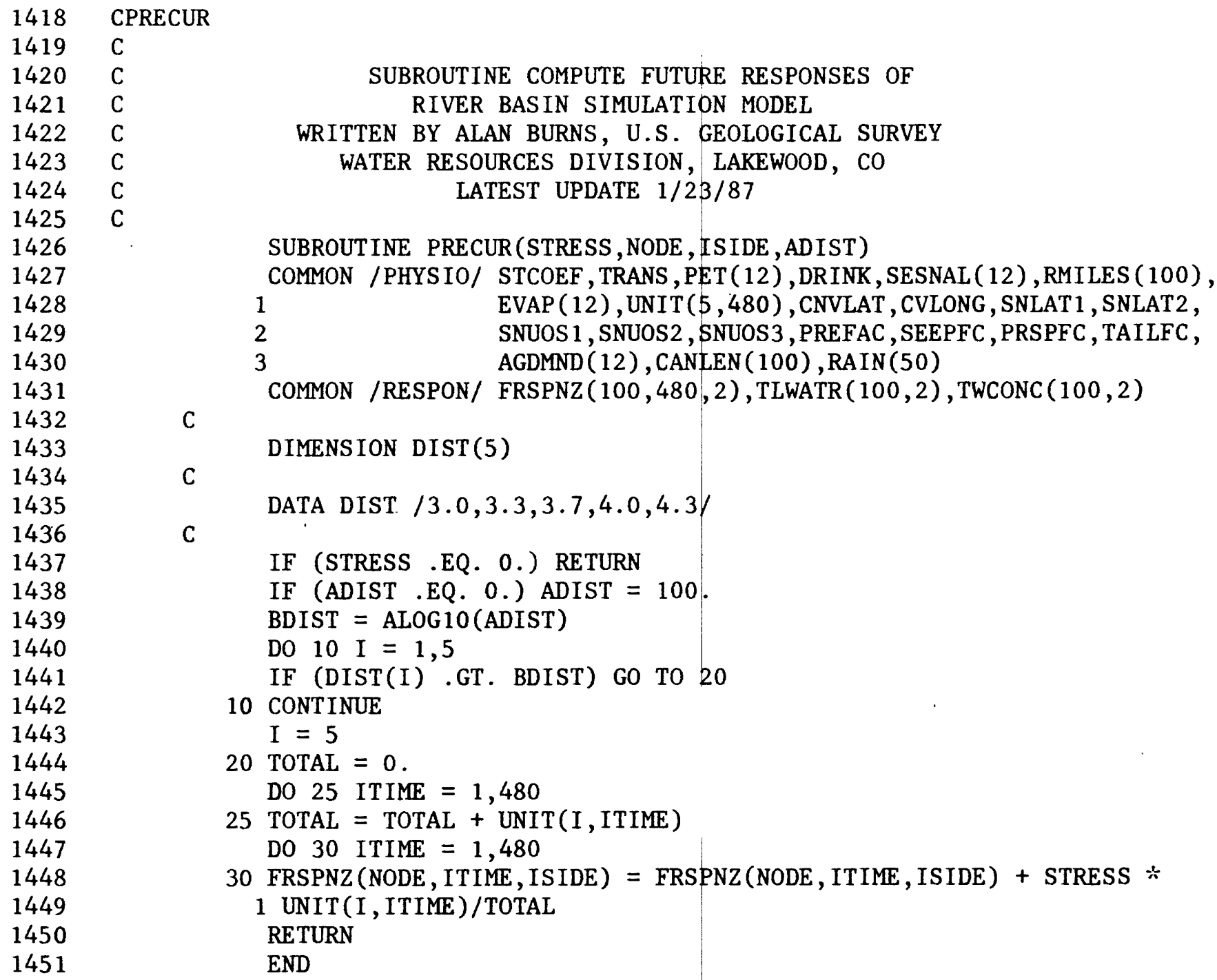


Attachment 8--FORTRAN Source Code of Subroutines

for Water-Supply Operations Simulation--Continued

\begin{tabular}{|c|c|c|}
\hline $\begin{array}{l}1452 \\
1453\end{array}$ & $\begin{array}{l}\text { CQUALTY } \\
\text { C }\end{array}$ & \\
\hline 1454 & C & SUBROUTINE WATER QUALITY OF RIVER BASIN SIMULATION MODEL \\
\hline 1455 & C & WRITTEN BY ALAN BURNS, U.S. GEOLOGICAL SURVEY \\
\hline 1456 & $\mathrm{C}$ & WATER RESOURCES DIVISION, LAKEWOOD, CO \\
\hline 1457 & $\mathrm{C}$ & LATEST UPDATE $9 / 11 / 87$ \\
\hline 1458 & C & \\
\hline 1459 & & SUBROUTINE QUALTY \\
\hline 1460 & & COMMON NMONTH, NINDV, IN, IOUT, IPRINT , IGRAPH, IBATCH, IQW, IMONTH, \\
\hline 1461 & & JMONTH, IYEAR \\
\hline 1462 & & COMMON /DEMND/ NUSERS,USERID (100), XLOCU (100), YLOCU (100), \\
\hline 1463 & & DMDFAC $(100), \operatorname{USRTYP}(100), \operatorname{NUMUSR}(100), \operatorname{NSOURC}(100)$ \\
\hline 1464 & & RFTYPE $(100), \operatorname{RFVAL}(100), \operatorname{RFXLOC}(100), \operatorname{RFYLOC}(100)$ \\
\hline 1465 & & $\operatorname{NODERF}(100), \operatorname{RFDIST}(100), \operatorname{ICODE}(100,15), \operatorname{IPRECP}(100)$ \\
\hline 1466 & & $\operatorname{QUANTY}(100,15), \operatorname{SCXIOC}(100,15), \operatorname{SCYLOC}(100,15)$ \\
\hline 1467 & & NODESC $(100,15), \operatorname{WANT}(100), \operatorname{NEED}(100), \operatorname{USXPLT}(100)$, \\
\hline 1468 & & USYPLT $(100), \operatorname{ISIDRF}(100), \operatorname{IAKNT}(100), \operatorname{ITIMFC}(100,15)$ \\
\hline 1469 & & TIMFAC $(100,12)$ \\
\hline 1470 & & INTEGER USERID, USRTYP, RFTYPE \\
\hline 1471 & & REAL NUMUSR, NEED \\
\hline 1472 & & COMMON /GW/ GWSTOR $(100,2), \operatorname{GWCONC}(100,2), \operatorname{RTRNFL}(100,2)$ \\
\hline 1473 & & COMMON /GWUSE/ MGW, GWUSER (100), GWSOUR(100), GWDIST (100), GWPUMP (100) \\
\hline 1474 & & $1 \quad, \operatorname{ISDPMP}(100)$ \\
\hline 1475 & & INTEGER GWUSER, GWSOUR \\
\hline 1476 & & COMMON /HYDGRG/ IHYGW(5), GWHYS $(600,5), \operatorname{GWHYC}(600,5), \operatorname{GWHYRF}(600,5)$ \\
\hline 1477 & & COMMON /HYDGRR/ IHYR (5), RHYS $(600,5), \operatorname{RHYC}(600,5)$ \\
\hline 1478 & & COMMON /HYDGRU/ IHYU (5), UHYD $(600,5), \operatorname{UHYP}(600,5), \operatorname{UHYT}(600,5)$ \\
\hline 1479 & & COMMON /REACHS/ NNODE, NODEID $(100)$, NXTNOD $(100)$, XLOC $(100)$, YLOC $(100)$, \\
\hline 1480 & & $1 \quad \operatorname{PLTX}(100), \operatorname{PLTY}(100), \operatorname{NTRCPT}(100,12), \operatorname{SLOPE}(100,12)$, \\
\hline 1481 & & $\mathrm{QWA}(100,12), \mathrm{QWB}(100,12), \operatorname{INDEPV}(100,12), \mathrm{Q}(100)$ \\
\hline 1482 & & $\mathrm{QW}(100), \operatorname{CONCFC}(100), \operatorname{NODEUS}(100), \mathrm{QSUBA}(100)$ \\
\hline 1483 & & QWSUBA $(100), \mathrm{DSA}(100), \mathrm{DSB}(100)$ \\
\hline 1484 & & REAL NTRCPT \\
\hline 1485 & & COMMON /PHYSIO/ STCOEF, TRANS, PET (12), DRINK, SESNAL (12), RMILES (100), \\
\hline 1486 & & $1 \quad \operatorname{EVAP}(12), \operatorname{UNIT}(5,480)$, CNVLAT , CVLONG , SNLAT 1, SNLAT2, \\
\hline 1487 & & SNUOS 1 , SNUOS 2 , SNUOS 3 , PREFAC, SEEPFC, PRSPFC, TAILFC, \\
\hline 1488 & & $\operatorname{AGDMND}(12)$, CANLEN $(100)$, RAIN $(50)$ \\
\hline 1489 & & COMMON /RESPON/ FRSPNZ $(100,480,2), \operatorname{TLWATR}(100,2), \operatorname{TWCONC}(100,2)$ \\
\hline 1490 & & COMMON /RESUSE / MRES, RESUSR (100), RESSOR (100), NUMRES (100), \\
\hline 1491 & & TRANSL (100), RESRL (100), ISBACT (100), RESQNT (100) \\
\hline 1492 & & INTEGER RESSOR,RESUSR \\
\hline 1493 & & COMMON /RESVOR/ NRES,RESSTR (25), RELEAS (25), NODRES (25), RESID (25), \\
\hline 1494 & & CAPMAX (25), NACTIV (25), AREAMX (25), RSXLOC (25), \\
\hline 1495 & & RSYLOC (25), RSCONC (25), RESEVP (25), RSXPLT (25), \\
\hline 1496 & & RSYPLT $(25), \operatorname{RESCUM}(25), \operatorname{SBSTOR}(25,5)$ \\
\hline 1497 & & INTEGER RESID \\
\hline 1498 & & REAL NACTIV \\
\hline 1499 & & COMMON /RES1/RESNAM(25) \\
\hline 1500 & & DOUBLE PRECISION RESNAM \\
\hline 1501 & & COMMON /STATR/ ISSR $(10), \operatorname{RMEANS}(10,12), \operatorname{RMEANC}(10,12), \operatorname{RMEANR}(10,12)$, \\
\hline 1502 & & RMEAND $(10,12), \operatorname{RMEANI}(10,12), \operatorname{RMEANE}(10,12)$ \\
\hline 1503 & & COMMON /TABLEG/ITBLG (5), IGTBLS $(5,600)$ I IGTBLC $(5,600), \operatorname{IGTBLR}(5,600)$ \\
\hline 1504 & & COMMON /TABLER/ ITBLR (5), IRTBLS $(5,600), \operatorname{IRTBLC}(5,600)$ \\
\hline 1505 & & COMMON /TABLEU/ ITBLU (5), IUTBLD $(5,600), \operatorname{IUTBLP}(5,600), \operatorname{IUTBLT}(5,600)$ \\
\hline
\end{tabular}


Attachment 8--FORTRAN Source Code of Subroutines

for Water-Supply Operations Simulation--Continued

1506

1507

1508

1509

1510

1511

1512

1513

1514

1515

1516

1517

1518

1519

1520

1521

1522

1523

1524

1525

1526

1527

1528

1529

1530

1531

1532

1533

1534

1535

1536

1537

1538

1539

1540

1541

1542

1543

1544

1545

1546

1547

1548

1549

1550

1551

1552

1553

1554

1555

1556

COMMON /USTATS/ DELIVR(100), RESREL(100), IMPORT (100), PUMPAG (100), 1 APCONC (100), DIVRZN (100), PUMPG $(100,2), \operatorname{CANLEK}(100)$,

2

REAL IMPORT

$\operatorname{DPPERC}(100)$, TAILWR (100), PRECIP(100)

$\mathrm{C}$

$\mathrm{C}$

DO 30 INODE $=1$, NNODE

IF (INDEPV (INODE, JMONTH) .GT. O) GO TO 20

DO 10 JNODE $=1$, INODE

IF (NXTNOD (JNODE) .LE. 0) GO TO 10 QSUBA (JNODE) $=0$.

10 CONTINUE

GO TO 30

$20 \mathrm{QW}($ INODE $)=0$.

$\mathrm{Q}(\mathrm{INODE})=0$.

30 CONTINUE

C

C

C

DO 150 INODE $=1$, NNODE

$\mathrm{QT}=\mathrm{Q}(\mathrm{INODE})+\mathrm{QSUBA}(\mathrm{INODE})$

IF (QT .LE. 0.) GO TO 40

$Q W($ INODE $)=(Q W(I N O D E) * Q(I N O D E)+Q W S U B A(I N O D E) * Q S U B A(I N O D E)) / Q T$

IF (QSUBA(INODE) .GE. O.) GO TO 40

$\mathrm{QMIN}=\mathrm{QW}(\mathrm{INODE}) \div \mathrm{QT} / \mathrm{Q}(\mathrm{INODE})$

$Q W(I N O D E)=Q W(I N O D E)-\operatorname{CONCFC}(I N O D E) *(Q W(I N O D E)-Q M I N)$

$40 \mathrm{IF}(\mathrm{QT}$. LT. O.) $\mathrm{QT}=0$.

$Q(I N O D E)=Q T$

C

DO 90 ISIDE $=1,2$

RTRN $=$ RTRNFL (INODE, ISIDE) $* 60.3$

IF (RTRN) $50,80,60$

$50 Q($ INODE $)=Q($ INODE $)+R T R N F L(I N O D E$, ISIDE $)$

IF (GWSTOR(INODE, ISIDE) .LT. .5) GO TO 55

GWCONC (INODE, ISIDE) $=(($ GWSTOR (INODE, IS IDE $)+$ RTRN $) *$

1 GWCONC (INODE, ISIDE) - RTRN $*$ QW(INODE)) / GWSTOR (INODE, ISIDE)

IF (GWCONC(INODE, ISIDE) .LT. .5) GWCONC (INODE, ISIDE) $=0$.

$54 \mathrm{IF}$ (Q(INODE) .LT. 0.) Q(INODE) $=0$.

GO TO 80

55 GWCONC (INODE, ISIDE) $=0$.

GO TO 54

$60 \mathrm{QT}=\mathrm{Q}(\mathrm{INODE})+\mathrm{RTRNFL}(\mathrm{INODE}, \mathrm{ISIDE})$

IF (QT .LE. O.) GO TO 70

$\mathrm{QW}(\mathrm{INODE})=(\mathrm{QW}(\mathrm{INODE}) \div \mathrm{Q}($ INODE $)+\mathrm{RTRNFL}(\mathrm{INODE}, \mathrm{ISIDE}) *$

1 GWCONC (INODE, ISIDE)) / QT

70 IF ( $($ (INODE) .LT. 0.) $Q(I N O D E)=0$.

$Q($ INODE $)=Q T$

$80 Q($ INODE $)=Q($ INODE $)+\operatorname{TLWATR}($ INODE, ISIDE $) / 60.3$

IF (Q (INODE) .EQ. O.) GO TO 90

$Q W($ INODE $)=(Q W($ INODE $) \div Q($ INODE $)+T W C O N C$ (INODE, ISIDE) $/ 60.3) / Q($ INODE $)$

90 CONTINUE 
Attachment 8--FORTRAN Source Code of Subroutines

for Water-Supply Operations Simulation--Continued

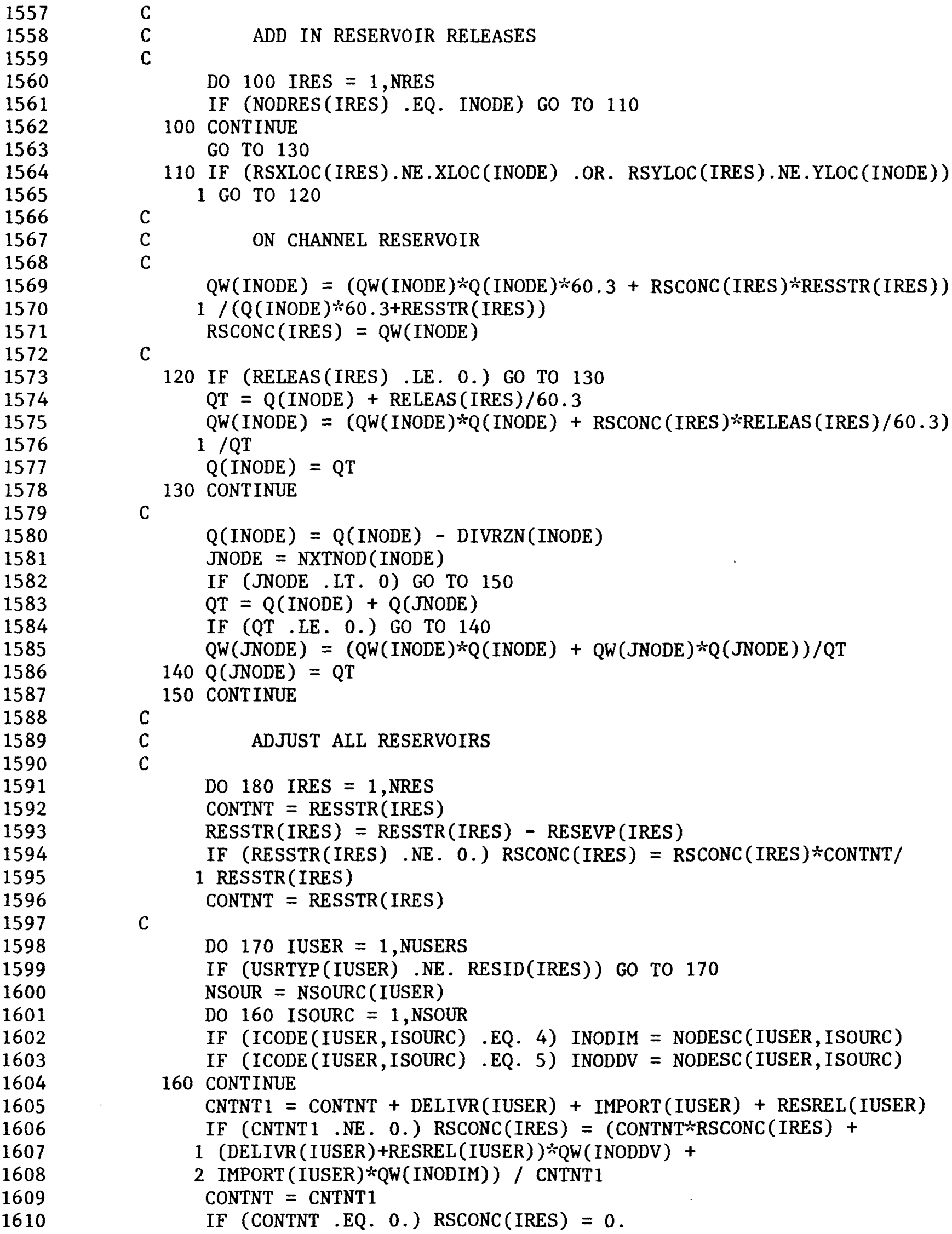


Attachment 8--FORTRAN Source Code of Subroutines

for Water-Supply Operations Simulation--Continued

1611

1612

1613

1614

1615

1616

1617

1618

1619

1620

1621

1622

1623

1624

1625

1626

1627

1628

1629

1630

1631

1632

1633

1634

1635

1636

1637

1638

1639

1640

1641

1642

1643

1644

1645

1646

1647

1648

1649

1650

1651

1652

1653

1654

1655

1656

1657

1658

1659

1660

1661

1662

1663
170 CONTINUE

RESSTR (IRES) $=$ CONTNT - RELEAS (IRES)

180 CONTINUE

C

C

C

DO 190 INODE $=1$, NNODE

DO 190 ISIDE $=1,2$

TLWATR (INODE, ISIDE) $=0$.

190 TWCONC (INODE, ISIDE) $=0$.

C

C COMPUTE WATER USE QUALITY

C

DO 330 IUSER $=1$, NUSERS

TLOAD $=0$

TAILWR (IUSER) $=0$.

DPPERC (IUSER) $=0$

NSOUR = NSOURC (IUSER)

DO 260 ISOURC $=1$, NSOUR

INODE = NODESC (IUSER, ISOURC)

GO TO $(200,210,230,250,200)$, ICODE (IUSER, ISOURC)

C

C

$\mathrm{C}$

200 QWDEL $=$ QW( INODE $)$

GO TO 260

C

C

C

210 DO $220 \mathrm{JGW}=1, \mathrm{MGW}$

IF (GWUSER(JGW) .NE. IUSER .OR. GWSOUR(JGW) .NE. ISOURC) GO TO 220 ISIDE = ISDPMP $(\mathrm{JGW})$

TLOAD $=$ TLOAD + GWPUMP (JGW)*GWCONC (INODE , ISIDE)

GWSTOR (INODE, ISIDE) = GWSTOR (INODE, ISIDE) - GWPUMP (JGW)

220 CONTINUE

GO TO 260

$\mathrm{C}$

C

C

230 DO 240 JRES $=1$, MRES

IF (RESUSR (JRES) .NE. IUSER .OR. RESSOR (JRES) .NE. ISOURC)

1 GO TO 240

IRES $=$ NUMRES (JRES)

QWRES = RSCONC (IRES)

IF (INODE . LE. NNODE) QWRES = QW(INODE)

TLOAD $=$ TLOAD + RESRL $(J R E S) * Q W R E S$

240 CONTINUE

GO TO 260

C

C

C

IMPORTS

250 QWIMP $=$ QW $($ INODE $)$

260 CONTINUE 
Attachment 8--FORTRAN Source Code of Subroutines

for Water-Supply Operations Simulation--Continued

1664

1665

1666

1667

1668

1669

1670

1671

1672

1673

1674

1675

1676

1677

1678

1679

1680

1681

1682

1683

1684

1685

1686

1687

1688

1689

1690

1691

1692

1693

1694

1695

1696

1697

1698

1699

1700

1701

1702

1703

1704

1705

1706

1707

1708

1709

1710

1711

1712

1713

1714

1715

1716

1717

1718

1719

C

CANLEK (IUSER) = AMIN1 (DELIVR (IUSER)+RESREL (IUSER),

1 SEEPFC*CANLEN (IUSER))

PRECIP (IUSER) $=0$.

TLOAD = TLOAD + DELIVR (IUSER) *QWDEL + IMPORT (IUSER) *QWIMP

1 - CANLEK (IUSER) $*$ QWDEL

TOTAL = DELIVR (IUSER) + PUMPAG (IUSER) + RESREL (IUSER)

$1+$ IMPORT (IUSER) - CANLEK (IUSER)

APCONC (IUSER) $=0$.

IF (TOTAL .NE. 0.) APCONC (IUSER) = TLOAD/TOTAL

C

$\mathrm{C}$

C

COMPUTE RETURN FLOW (TAILWATER AND DEEP PERCOLATION)

ISIDE = ISIDRF (IUSER)

INODE = NODERF (IUSER)

IP = IPRECP (IUSER)

IF (IP .NE . 0) EFPRCP = AMIN1 (PRSPFC, RAIN(IP))

IF (IP .EQ. 0) EFPRCP $=0$.

PRECIP (IUSER) $=$ NUMUSR (IUSER) *EFPRCP $/ 12$.

TOTAL = TOTAL + PRECIP (IUSER)

IF (TOTAL .NE. 0.) APCONC(IUSER) = TLOAD/TOTAL

USED = AMIN1 (TOTAL, NEED (IUSER))

RTRN = TOTAL - USED

GO TO $(290,280,280,270,270)$, RFTYPE (IUSER)

270 RTRN = RTRN*RFVAL (IUSER)

TLOAD $=$ TLOAD *RFVAL (IUSER)

GO TO 290

280 RTRN $=$ TOTAL $*$ RFVAL $($ IUSER $)$

290 RTRN $=$ RTRN + CANLEK (IUSER)

TLOAD $=$ TLOAD + CANLEK $($ IUSER $) *$ QWDEL

IF (RTRN .LE. 0.) GO TO 330

TAILWR(IUSER) $=0$.

GO TO $(300,310,320,310,320)$, RFTYPE (IUSER)

$300 \operatorname{TAILWR}($ IUSER) $=\operatorname{RTRN} *(1$. RFVAL (IUSER) $)$

TWLOAD $=$ TLOAD $*(1 .-$ RFVAL (IUSER) $)$

TLOAD = TLOAD *RFVAL (IUSER)

GO TO 320

310 TAILWR (IUSER) $=$ RTRN

TWLOAD = TLOAD

TLOAD $=0$.

320 DPPERC (IUSER) = RTRN - TAILWR (IUSER)

TLWATR (INODE, ISIDE) = TLWATR (INODE, ISIDE) + TAILWR (IUSER) $*$ TAILFC

FRSPNZ (INODE , 1, ISIDE) = FRSPNZ (INODE , 1 , ISIDE) +

1 TAILWR (IUSER) *(1. -TAILFC)

CALL CMPUTR (DPPERC (IUSER), INODE, ISIDE, RFDIST (IUSER))

GWSTR = GWSTOR (INODE, ISIDE) + DPPERC (IUSER) +

1 TAILWR (IUSER) * (1. -TAILFC)

IF (GWSTR . NE. O.) GWCONC (INODE, ISIDE) $=$ (GWSTOR (INODE, ISIDE)*

1 GWCONC (INODE, ISIDE) + TLOAD + TWLOAD $\div$ (1. -TAILFC)) /GWSTR

GWSTOR (INODE, ISIDE) $=$ GWSTR

IF (GWCONC (INODE, ISIDE) . LT. .5) GWCONC (INODE, ISIDE) $=0$.

IF (GWSTOR (INODE, ISIDE) .LT. .5) GWCONC (INODE, ISIDE) $=0$.

330 CONTINUE

C

RETURN

END 
Attachment 8--FORTRAN Source Code of Subroutines

for Water-Supply Operations Simulation--Continued

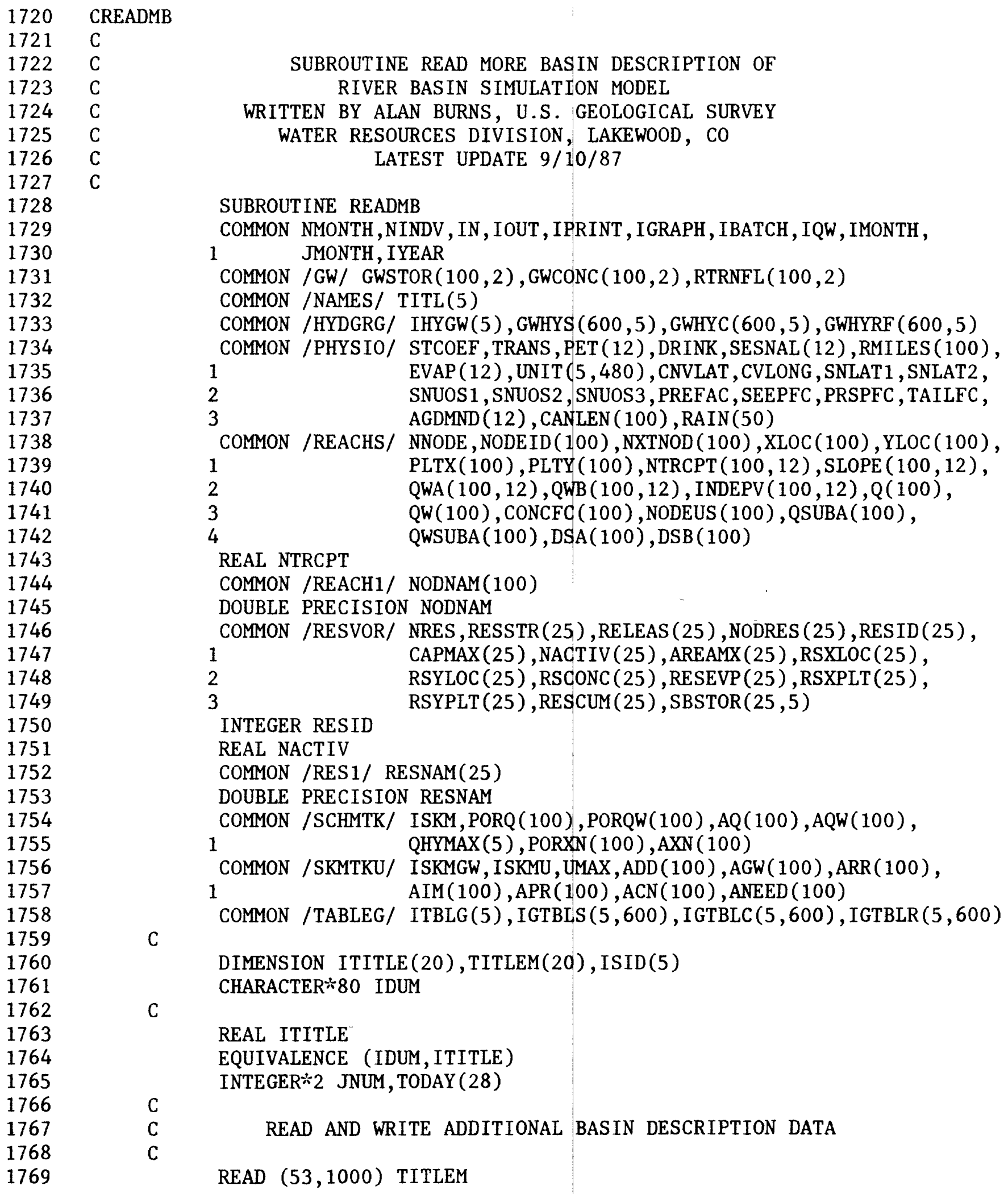


Attachment 8--FORTRAN Source Code of Subroutines

for Water-Supply Operations Simulation--Continued

1770

1771

1772

1773

1774

1775

1776

1777

1778

1779

1780

1781

1782

1783

1784

1785

1786

1787

1788

1789

1790

1791

1792

1793

1794

1795

1796

1797

1798

1799

1800

1801

1802

1803

1804

1805

1806

1807

1808

1809

1810

1811

1812

1813

1814

1815

1816

1817

1818

1819

1820
1000 FORMAT (20A4)

$\mathrm{C}$

JNUM $=28$

CALL TIMDAT(TODAY, JNUM)

WRITE $(52,1010)$ TITL, (TODAY (JNUM) , JNUM=1,3), TITLEM, NNODE

1010 FORMAT (82H1 BSNMD3 - RIVER BASIN SIMULATION MODEL 非3

1D $9 / 30 / 85$ BY ALAN BURNS ,///,20H SIMULATION TITLE: ,5A4,

2 66X,10H DATE RUN: ,A2,1H/,A2,1H/,A2,///,

$337 \mathrm{H}$ ADDITIONAL BASIN DESCRIPTION FILE: ,20A4,6H, HAS , I3,

$46 \mathrm{H}$ NODES )

$\mathrm{C}$

READ $(53,1080)$ PET

WRITE $(52,1020)$ PET

1020 FORMAT $(/ / /, 55 \mathrm{H}$ POTENTIAL EVAPOTRANSPIRATION (PET), IN FEET PER M 1ONTH ,/ 5X,12F6.2)

READ $(53,1080)$ EVAP

WRITE $(52,1030)$ EVAP

1030 FORMAT $(/ / /, 36 \mathrm{H}$ PAN EVAPORATION, IN FEET PER MONTH,/,5X,12F6.2)

READ $(53,1080)$ AGDMND

WRITE $(52,1040)$ AGDMND

1040 FORMAT $(/ / /, 51 \mathrm{H}$ AGRICULTURAL IRRIGATION DEMAND, IN FEET PER MONTH 1 ,/ $5 \mathrm{X}, 12 \mathrm{~F} 6.2)$

READ $(53,1080)$ SESNAL

WRITE $(52,1050)$ SESNAL

1050 FORMAT $(/ / /, 46 \mathrm{H}$ SEASONAL IN-STREAM DEMAND, IN. FEET PER MONTH

1 ,/ $5 \mathrm{X}, 12 \mathrm{~F} 6.2)$

READ $(53,1080)$ STCOEF, TRANS

WRITE $(52,1060)$ STCOEF, TRANS

1060 FORMAT $(/ / /, 50 \mathrm{H}$ AVERAGE BASIN STORAGE COEFFICIENT, IN PERCENT = , 1 F5.2,/,58H AVERAGE BASIN TRANSMISSIVITY, IN FEET SQUARED PER DAY $2=, \mathrm{F} 8.0)$

READ $(53,1080)$ DRINK

WRITE $(52,1070)$ DRINK

1070 FORMAT $(/ / /, 52 \mathrm{H}$ MUNICIPAL USAGE, IN ACRE-FEET PER UNIT QUANTITY = $1, \mathrm{~F} 8.2$ )

READ $(53,1080)$ CNVLAT, CVLONG, SNUOS1, SNUOS2, SNUOS3, SNLAT1, SNLAT2,

1 PREFAC, SEEPFC, PRSPFC, TAILFC

1080 FORMAT $(8 \mathrm{X}, 12 \mathrm{~F} 6.0)$

WRITE $(52,1090)$ PREFAC

1090 FORMAT $(/ / /, 33 \mathrm{H}$ PRESTRESS FACTOR, IN PERCENT $=$,F5.2)

WRITE $(52,1100)$ SEEPFC

1100 FORMAT $(/ / /, 48 \mathrm{H}$ CANAL LEAKAGE FACTOR, IN ACRE-FEET PER MILE = ,

$1 \mathrm{~F} 5.2$ )

WRITE $(52,1110)$ PRSPFC

1110 FORMAT $(/ / /, 39 \mathrm{H}$ EFFECTIVE PRECIPITATION, IN INCHES $=$,F5.2)

C

C

C

READ AND WRITE RESERVOIR DATA

READ $(53,1120)$ NRES

1120 FORMAT (I5)

WRITE $(52,1130)$ NRES 
Attachment 8--FORTRAN Source Code of Subroutines

for Water-Supply Operations Simulation--Continued

1821

1822

1823

1824

1825

1826

1827

1828

1829

1830

1831

1832

1833

1834

1835

1836

1837

1838

1839

1840

1841

1842

1843

1844

1845

1846

1847

1848

1849

1850

1851

1852

1853

1854

1855

1856

1857

1858

1859

1860

1861

1862

1863

1864

1865

1866

1867

1130 FORMAT (11H1THERE ARE ,I3,11H RESERVOIRS ,//, 11 108H RESERVOIR RESERVOIR RESERVOIR MAXIMUM INACTIVE M 2AXIMUM $X \quad Y$ CONNECTING INITIAL INITIAL ,/, $111 \mathrm{H} N$ 3UMBER ID NAME CAPACITY STORAGE SURFACE COORD 4COORD RIVER CONTENTS CONCENTRATION ,/,35X,75H(ACRE- (ACR 5E- AREA (DE- (DE- NODE (ACRE- (MILLIGRAMS , /, 6 36X, 39HFEET) FEET) (ACRES) GREES) GREES), 15X, 20HFEET) PE 7R LITER) )

DO 20 IRES $=1$, NRES

READ $(53,1140)$ RESID (IRES), RESNAM (IRES), CAPMAX (IRES), NACTIV (IRES), 1 AREAMX (IRES), RSXLOC (IRES), RSYLOC (IRES), RESSTR (IRES), RSCONC (IRES),

2 RSXPLT (IRES), RSYPLT (IRES)

1140 FORMAT (I5, A8, 2X, 7F 10.0,2F5.0)

CALL DETNOD(RSXLOC (IRES), RSYLOC (IRES), NODRES (IRES), ISIDE)

SBSTOR(IRES, 1) = RESSTR(IRES) - NACTIV(IRES)

DO 10 IACCNT $=2,5$

10 SBSTOR (IRES, IACCNT) $=0$.

WRITE $(52,1150)$ IRES, RESID(IRES), RESNAM(IRES), CAPMAX(IRES),

1 NACTIV(IRES), AREAMX (IRES), RSXLOC (IRES), RSYLOC (IRES), NODRES (IRES),

2 RESSTR (IRES), RSCONC (IRES)

1150 FORMAT (I6,6X,I6,6X, A8,F9.0,F10.0,F8.0,F9.3, F7.3, I6, F14.0, F12.0)

20 CONTINUE

C

C

C

\section{PLOT RESERVOIR LOCATIONS}

CALL QUESZN(IQ)

IF $($ IQ- 1$) \quad 40,30,40$

30 WRITE (IDUM, 1160) TITL

1160 FORMAT (5A4,23H - RESERVOIR LOCATIONS\$)

CALI PLTRES (ITITLE)

C

$\mathrm{C}$

$\mathrm{C}$

40 DO 50 INODE $=1$, NNODE

50 RMILES (INODE) $=0$.

INODE = NNODE

RMILES (INODE) $=.00001$

60 JNODE = NODEUS (INODE)

IF (JNODE .EQ. 0) GO TO 70

SNUOSY $=$ SNUOS 1

IF (XLOC (JNODE) .GT. SNLAT1) SNUOSY $=$ SNUOS2

IF (XLOC (JNODE) .GT. SNLAT2) SNUOSY $=$ SNUOS3

RMILES (JNODE) = RMILES (INODE) +

$1 \mathrm{SQRT}(((\mathrm{XLOC}$ (JNODE)-XLOC (INODE) )*CVLONG)**2 + ((YLOC (JNODE) -

2 YLOC (INODE) )*CNVLAT) $* * 2) *$ SNUOSY

INODE = JNODE

GO TO 60 
Attachment 8--FORTRAN Source Code of Subroutines

for Water-Supply Operations Simulation--Continued

1868

1869

1870

1871

1872

1873

1874

1875

1876

1877

1878

1879

1880

1881

1882

1883

1884

1885

1886

1887

1888

1889

1890

1891

1892

1893

1894

1895

1896

1897

1898

1899

1900

1901

1902

1903

1904

1905
70 IZERO $=0$

NNOD1 $=$ NNODE -1

DO 80 INODE $=1$, NNOD 1

IF (RMILES (INODE) .NE. O.) GO TO 80

JNODE = NXTNOD (INODE)

IF (JNODE .LE. 0) GO TO 80

IZERO $=1$

IF (RMILES (JNODE) .EQ. 0.) GO TO 80

SNUOSY $=$ SNUOS 1

IF (XLOC (INODE) .GT. SNLAT 1) SNUOSY $=$ SNUOS2

IF (XLOC (INODE) . GT . SNLAT2) SNUOSY $=$ SNUOS3

RMILES (INODE) = RMILES $(J N O D E)+$

1 SQRT (( (XLOC (INODE) -XLOC (JNODE)) *CVLONG) $\div \div 2$ + ((YLOC (INODE) -

2 YLOC (JNODE ) $) *$ CNVLAT) $* * 2) *$ SNUOSY

80 CONTINUE

IF (IZERO .EQ. 1) GO TO 70

C

$\mathrm{C}$

$\mathrm{C}$

READ AND WRITE GROUND-WATER DATA

WRITE $(52,1170)$

1170 FORMAT (68H1 NODE NODE NODE INITIAL GROUND- INITIAL GROU IND- RIVER,/,68H NUMBER ID NAME WATER STORAGE WATER 2CONCENTRATION LENGTH , /,27X,42H(ACRE-FEET) (MILLIGRAMS 3 (MILES) ,/,47X, 10HPER LITER) ,/, 25X,33HSIDE 1 SIDE 2 SIDE 1 4 SIDE 2 )

DO 90 INODE $=1$, NNODE

READ $(53,1080$ ) (GWSTOR(INODE, ISIDE), GWCONC (INODE, ISIDE), ISIDE=1 ,2)

WRITE $(52,1180$ ) INODE, NODE ID (INODE), NODNAM (INODE), GWSTOR (INODE , 1),

1 GWSTOR (INODE , 2) , GWCONC (INODE , 1), GWCONC (INODE , 2), RMILES (INODE)

1180 FORMAT (I5, I8, 2X, A8, 2F8.0,F9.0,F10.0,F9.1)

90 CONTINUE

C

C

C

READ (IN, 1190) ISKMGW

1190 FORMAT (16I5)

C

C

ENTER NODES AND SIDE FOR GROUND-WATER HYDROGRAPHS 


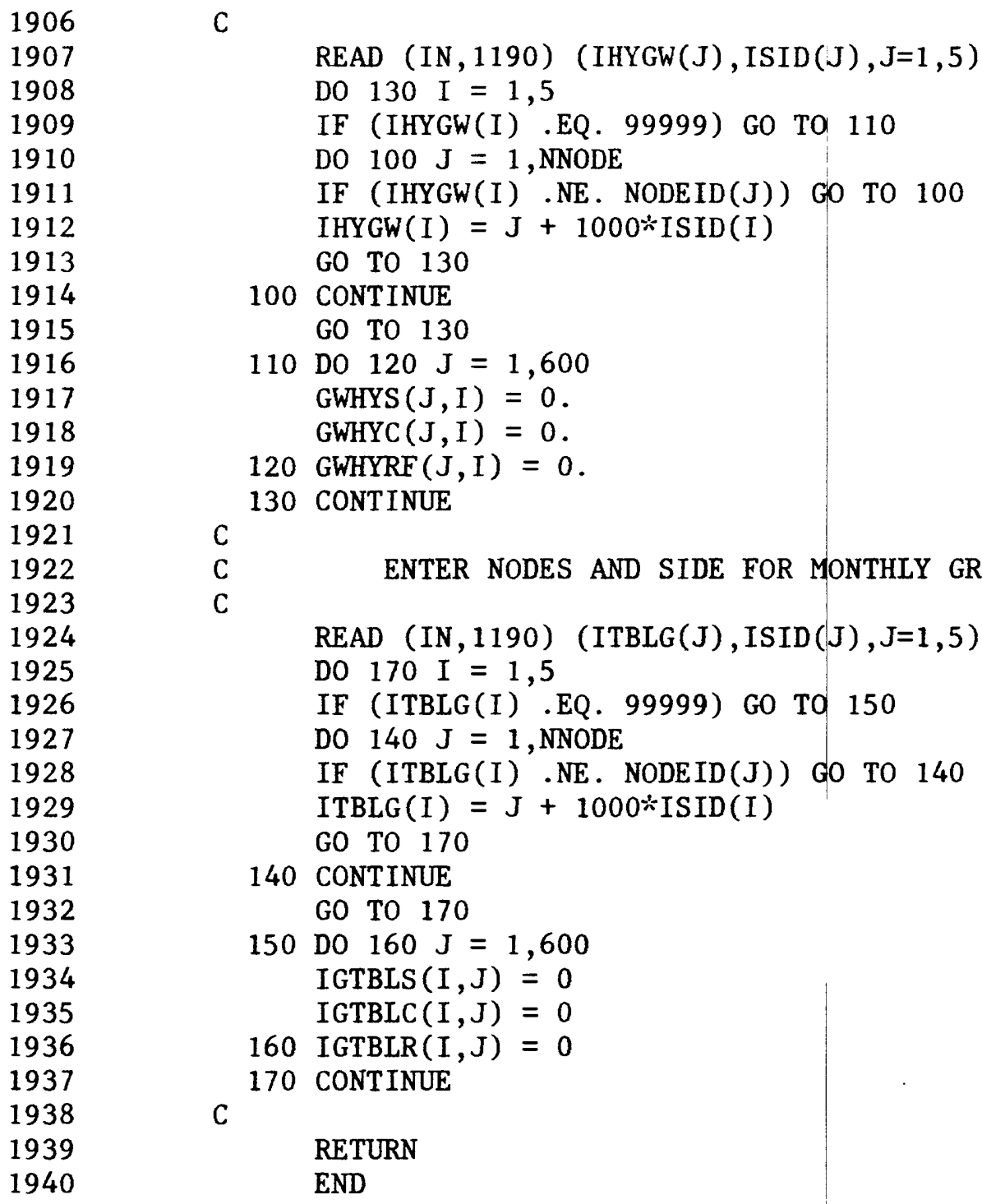

C

$\operatorname{READ}(\operatorname{IN}, 1190)(\operatorname{IHYGW}(\mathrm{J}), \operatorname{ISID}(\mathrm{J}), \mathrm{J}=1,5)$

DO $130 \mathrm{I}=1,5$

IF (IHYGW(I) .EQ. 99999) GO TO 110

DO $100 \mathrm{~J}=1$, NNODE

IF (IHYGW(I) .NE. NODEID(J)) GO TO 100

$\operatorname{IHYGW}(I)=J+1000 * \operatorname{ISID}(\mathrm{I})$

GO TO 130

100 CONTINUE

GO TO 130

110 Do $120 \mathrm{~J}=1,600$

$\operatorname{GWHYS}(J, I)=0$.

$\operatorname{GWHYC}(J, I)=0$.

$120 \operatorname{GWHYRF}(\mathrm{J}, \mathrm{I})=0$.

130 CONTINUE

C

C

C

$\operatorname{READ}(\operatorname{IN}, 1190)(\operatorname{ITBLG}(J), \operatorname{ISID}(J), J=1,5)$

DO $170 \quad I=1,5$

IF (ITBLG(I) .EQ. 99999) GO TO 150

DO $140 \mathrm{~J}=1$, NNODE

IF (ITBLG(I) .NE. NODEID(J)) GO TO 140

$\operatorname{ITBLG}(I)=\mathrm{J}+1000 * \operatorname{ISID}(\mathrm{I})$

GO TO 170

140 CONTINUE

GO TO 170

$150 \mathrm{DO} 160 \mathrm{~J}=1,600$

$\operatorname{IGTBLS}(I, J)=0$

$\operatorname{IGTBLC}(I, J)=0$

$160 \operatorname{IGTBLR}(I, J)=0$

170 CONTINUE

C

RETURN

END 
Attachment 8--FORTRAN Source Code of Subroutines

for Water-Supply Operations Simulation--Continued

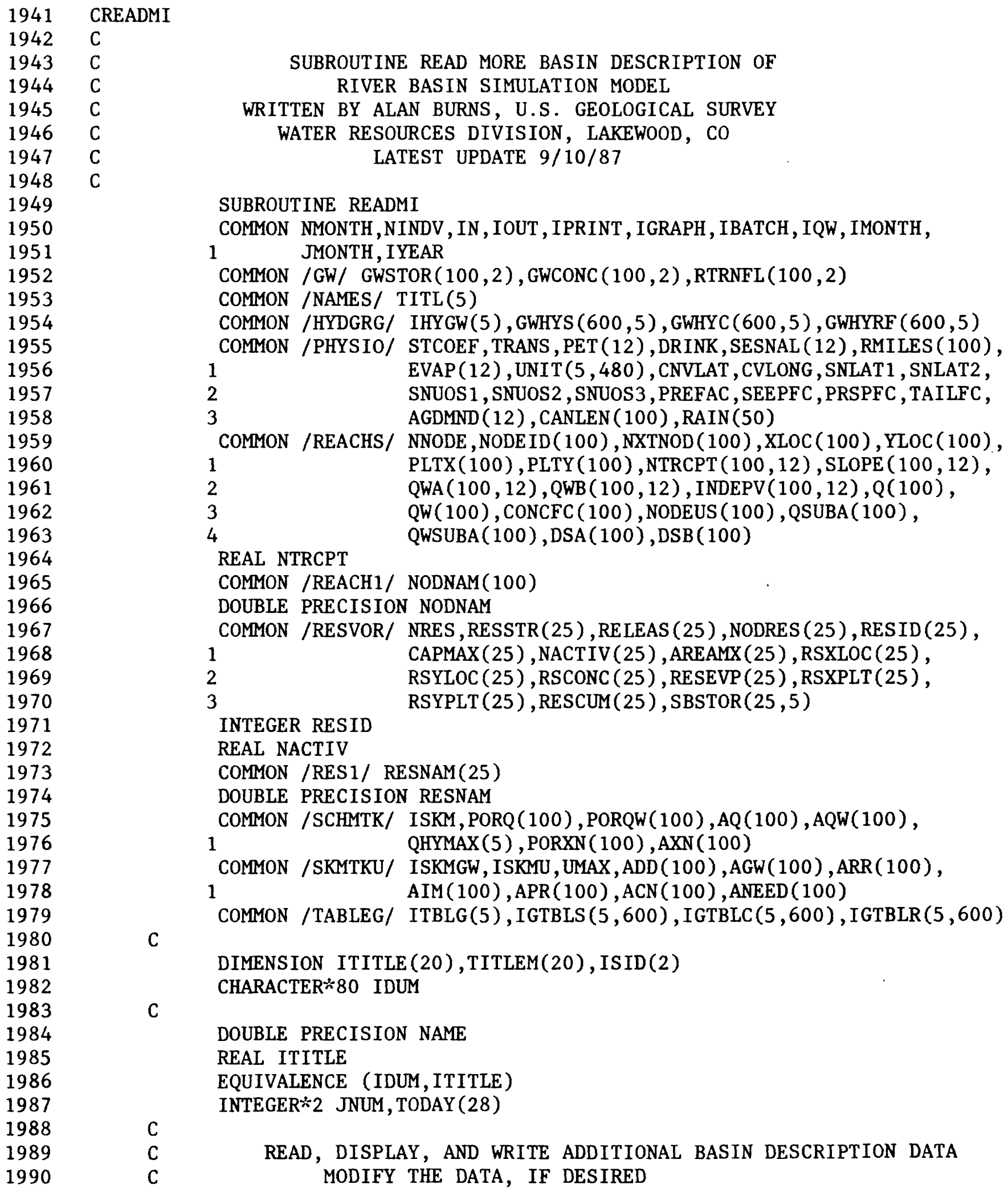

READ, DISPLAY, AND WRITE ADDITIONAL BASIN DESCRIPTION DATA MODIFY THE DATA, IF DESIRED 
Attachment 8--FORTRAN Source Code of Subroutines

for Water-Supply Operations Simulation--Continued

1991

1992

1993

1994

1995

1996

1997

1998

1999

2000

2001

2002

2003

2004

2005

2006

2007

2008

2009

2010

2011

2012

2013

2014

2015

2016

2017

2018

2019

2020

2021

2022

2023

2024

2025

2026

2027

2028

2029

2030

2031

2032

2033

2034

2035

2036

2037

2038

2039

2040

2041

2042
C

READ $(53,1000)$ TITLEM

1000 FORMAT (20A4)

JNUM $=28$

CALL TIMDAT (TODAY, JNUM)

WRITE $(52,1010)$ TITL, (TODAY (JNUM) , JNUM=1, 3), TITLEM, NNODE

1010 FORMAT (82H1 BSNMD3 - RIVER BASIN SIMULATION MODEL 非3

1D $9 / 30 / 85$ BY ALAN BURNS, ///,20H SIMULATION TITLE: ,5A4,

2 66X,10H DATE RUN: ,A2,1H/,A2,1H/,A2,///,

$337 \mathrm{H}$ ADDITIONAL BASIN DESCRIPTION FILE: ,20A4,6H, HAS , I3,

4 6H NODES )

C

WRITE (IOUT, 1020)

1020 FORMAT (56H DO YOU WANT THE ADDITIONAL BASIN DESCRIPTION DISPLAYED $1 ?$ )

CALL QUESZN(IPRNT)

IF (IPRNT .EQ. 1) WRITE (IOUT, 1030)

1030 FORMAT (55H THE ADDITIONAL BASIN DESCRIPTION FILE YOU ATTACHED IS:

$1, /, 20 \mathrm{~A} 4)$

C

READ $(53,1160)$ PET

IF (IPRNT .NE. 1) GO TO 30

WRITE (IOUT, 1060) PET

10 WRITE (IOUT, 1040)

1040 FORMAT (54H TO CHANGE ANY VALUE, ENTER MONTH NUMBER AND NEW VALUE) READ (IN, 1050) J,PETJ

1050 FORMAT (I2, F10.0)

IF (J) $30,30,20$

$20 \operatorname{PET}(\mathrm{J})=\operatorname{PETJ}$

GO TO 10

30 WRITE $(52,1060)$ PET

1060 FORMAT $(/ / /, 55 \mathrm{H}$ POTENTIAL EVAPOTRANSPIRATION (PET), IN FEET PER M 1ONTH ,/ $5 \mathrm{X}, 12 \mathrm{~F} 6.2)$

READ $(53,1160)$ EVAP

IF (IPRNT .NE. 1) GO TO 60

WRITE (IOUT, 1070) EVAP

40 WRITE (IOUT, 1040)

READ. (IN, 1050) J,EVAPJ

IF (J) $60,60,50$

$50 \operatorname{EVAP}(\mathrm{J})=\operatorname{EVAPJ}$

GO TO 40

60 WRITE $(52,1070)$ EVAP

1070 FORMAT $(/ / /, 36 \mathrm{H}$ PAN EVAPORATION, IN FEET PER MONTH, /,5X,12F6.2)

READ $(53,1160)$ AGDMND

IF (IPRNT .NE. 1) GO TO 90

WRITE (IOUT, 1080) AGDMND

70 WRITE (IOUT, 1040)

READ (IN, 1050) J,AGDMDJ

IF (J) $90,90,80$

$80 \operatorname{AGDMND}(\mathrm{J})=\operatorname{AGDMDJ}$

GO TO 70

90 WRITE $(52,1080)$ AGDMND 
Attachment 8--FORTRAN Source Code of Subroutines

for Water-Supply Operations Simulation--Continued

2043

2044

2045

2046

2047

2048

2049

2050

2051

2052

2053

2054

2055

2056

2057

2058

2059

2060

2061

2062

2063

2064

2065

2066

2067

2068

2069

2070

2071

2072

2073

2074

2075

2076

2077

2078

2079

2080

2081

2082

2083

2084

2085

2086

2087

2088

2089

2090

2091

2092

2093

2094

2095

1080 FORMAT $(/ / /, 51 \mathrm{H}$ AGRICULTURAL IRRIGATION DEMAND, IN FEET PER MONTH 1 ,/ $5 \mathrm{X}, 12 \mathrm{~F} 6.2)$

READ $(53,1160)$ SESNAL

IF (IPRNT .NE. 1) GO TO 120

WRITE (IOUT, 1090) SESNAL

100 WRITE (IOUT, 1040)

READ (IN, 1050) J, SESNLJ

IF (J) $120,120,110$

$110 \operatorname{SESNAL}(J)=\operatorname{SESNL} J$

GO TO 100

120 WRITE $(52,1090)$ SESNAL

1090 FORMAT $(/ / /, 46 \mathrm{H}$ SEASONAL IN-STREAM DEMAND, IN FEET PER MONTH

$1, / 5 \mathrm{X}, 12 \mathrm{~F} 6.2)$

READ $(53,1160)$ STCOEF, TRANS

IF (IPRNT .NE. 1) GO TO 130

WRITE (IOUT, 1130) STCOEF, TRANS

WRITE (IOUT, 1100)

1100 FORMAT (33H DO YOU WANT TO ENTER NEW VALUES?)

CALL QUESZN(IQ)

IF (IQ .NE. 1) GO TO 130

WRITE (IOUT, 1110)

1110 FORMAT (71H ENTER STORAGE COEFFICIENT (10 COLUMNS) AND TRANSMISSIV

IITY ( 10 COLUMNS))

READ (IN, 1120) STCOEF, TRANS

1120 FORMAT (2F10.0)

130 WRITE $(52,1130)$ STCOEF, TRANS

1130 FORMAT $(/ / /, 50 \mathrm{H}$ AVERAGE BASIN STORAGE COEFFICIENT, IN PERCENT = ,

1 F5.2,/,58H AVERAGE BASIN TRANSMISSIVITY, IN FEET SQUARED PER DAY $2=, F 8.0)$

READ $(53,1160)$ DRINK

IF (IPRNT .NE. 1) GO TO 140

WRITE (IOUT, 1150) DRINK

WRITE (IOUT, 1100)

CALL QUESZN(IQ)

IF (IQ .NE. 1) GO TO 140

WRITE (IOUT, 1140)

1140 FORMAT (44H ENTER AVERAGE PER PERSON USAGE (10 COLUMNS) ) READ (IN, 1120) DRINK

140 WRITE $(52,1150)$ DRINK

1150 FORMAT $(/ / /, 52 \mathrm{H}$ MUNICIPAL USAGE, IN ACRE-FEET PER UNIT QUANTITY = 1, F8.2)

READ $(53,1160)$ CNVLAT, CVLONG , SNUOS 1 , SNUOS2 , SNUOS3, SNLAT1, SNLAT2,

1 PREFAC, SEEPFC, PRSPFC, TAILFC

1160 FORMAT $(8 \mathrm{X}, 12 \mathrm{~F} 6.0)$

IF (IPRNT .NE. 1) GO TO 150

WRITE (IOUT, 1180) PREFAC

WRITE (IOUT, 1100)

CALL QUESZN (IQ)

IF (IQ .NE. 1) GO TO 150

WRITE (IOUT, 1170)

1170 FORMAT (36H ENTER PRESTRESS FACTOR (10 COLUMNS) )

READ (IN, 1120) PREFAC

150 WRITE $(52,1180)$ PREFAC 
Attachment 8--FORTRAN Source Code of Subroutines

for Water-Supply Operations Simulation--Continued

2096

2097

2098

2099

2100

2101

2102

2103

2104

2105

2106

2107

2108

2109

2110

2111

2112

2113

2114

2115

2116

2117

2118

2119

2120

2121

2122

2123

2124

2125

2126

2127

2128

2129

2130

2131

2132

2133

2134

2135

2136

2137

2138

2139

2140

2141

2142

2143

2144

2145

2146

2147

2148

1180 FORMAT $(/ / /, 33 \mathrm{H}$ PRESTRESS FACTOR, IN PERCENT $=$, F5.2)

IF (IPRNT .NE. 1) GO TO 160

WRITE (IOUT, 1200) SEEPFC

WRITE (IOUT, 1100)

CALL QUESZN(IQ)

IF (IQ .NE. 1) GO TO 160

WRITE (IOUT, 1190)

1190 FORMAT (34H ENTER SEEPAGE FACTOR ( 10 COLUMNS) )

READ (IN, 1120) SEEPFC

160 WRITE $(52,1200)$ SEEPFC

1200 FORMAT $(/ / /, 48 \mathrm{H}$ CANAL LEAKAGE FACTOR, IN ACRE-FEET PER MILE = , $1 \mathrm{~F} 5.2$ )

IF (IPRNT .NE. 1) GO TO 170

WRITE (IOUT, 1220) PRSPFC

WRITE (IOUT, 1100)

CALL QUESZN(IQ)

IF (IQ .NE. 1) GO TO 170

WRITE (IOUT, 1210)

1210 FORMAT (50H ENTER EFFECTIVE PRECIPITATION FACTOR (10 COLUMNS) )

READ (IN , 1120) PRSPFC

170 WRITE $(52,1220)$ PRSPFC

1220 FORMAT $(/ / /, 39 \mathrm{H}$ EFFECTIVE PRECIPITATION, IN INCHES = ,F5.2)

C

C READ AND DISPLAY RESERVOIR DATA

$\mathrm{C}$

READ $(53,1230)$ NRES

1230 FORMAT (2I5)

C

WRITE (IOUT, 1240)

1240 FORMAT (43H DO YOU WANT TO DISPLAY THE RESERVOIR DATA? )

CALL QUESZN(IQ)

IF (IQ .EQ. 1) WRITE (IOUT, 1250) NRES

1250 FORMAT (11H1THERE ARE , I3,11H RESERVOIRS ,//, 1 108H RESERVOIR RESERVOIR RESERVOIR MAXIMUM INACTIVE M 2AXIMUM $X \quad Y$ CONNECTING INITIAL INITIAL , /, $111 \mathrm{H} N$ 3UMBER ID NAME CAPACITY STORAGE SURFACE COORD 4COORD RIVER CONTENTS CONCENTRATION ,/,35X,75H(ACRE- (ACR 5E- AREA (DE- (DE- NODE (ACRE- (MILLIGRAMS ,/, 6 36X, 39HFEET) FEET) (ACRES) GREES) GREES), 15X, 20HFEET) PE 7R LITER) ) DO 190 IRES $=1$, NRES

READ (53, 1260) RESID(IRES), RESNAM(IRES), CAPMAX (IRES), NACTIV(IRES), 1 AREAMX (IRES), RSXIOC (IRES), RSYLOC (IRES), RESSTR (IRES), RSCONC (IRES), 2 RSXPLT (IRES), RSYPLT (IRES)

1260 FORMAT (I5, A8, 2X, 7F 10.0,2F5.0)

CALL DETNOD (RSXLOC (IRES), RSYLOC (IRES), NODRES (IRES), ISIDE)

SBSTOR (IRES, 1) = RESSTR (IRES) - NACTIV (IRES)

DO 180 IACCNT $=2,5$

$180 \operatorname{SBSTOR}$ (IRES, IACCNT) $=0$.

IF (IQ .NE. 1) GO TO 190

WRITE (IOUT , 1270) IRES, RESID(IRES), RESNAM(IRES), CAPMAX (IRES), 1 NACTIV(IRES), AREAMX (IRES), RSXLOC (IRES), RSYLOC (IRES), NODRES (IRES), 2 RESSTR (IRES), RSCONC (IRES) 
Attachment 8--FORTRAN Source Code of Subroutines

for Water-Supply Operations Simulation--Continued

2149

2150

2151

2152

2153

2154

2155

2156

2157

2158

2159

2160

2161

2162

2163

2164

2165

2166

2167

2168

2169

2170

2171

2172

2173

2174

2175

2176

2177

2178

2179

2180

2181

2182

2183

2184

2185

2186

2187

2188

2189

2190

2191

2192

2193

2194

2195

2196

2197

2198

2199

2200

2201

2202

2203
1270 FORMAT (I6,6X, I6,6X, A8, F9.0,F10.0,F8.0,F9.3,F7.3, I6,F14.0,F 12.0)

190 CONTINUE

C

C

C

200 WRITE (IOUT, 1280)

1280 FORMAT (42H DO YOU WANT TO MODIFY THE RESERVOIR DATA? )

CALL QUESZN (IQ)

$\mathrm{C}$ IF (IQ - 1) $360,210,360$

C ADD A NEW RESERVOIR

C

210 WRITE (IOUT, 1290)

1290 FORMAT (32H DO YOU WANT TO ADD A RESERVOIR? )

CALL QUESZN (IQ)

IF (IQ - 1) $250,220,250$

220 NRES $=$ NRES +1

IRES $=$ NRES

230 WRITE (IOUT, 1300)

1300 FORMAT (33H ENTER THE RESERVOIR ID (5 COLS) )

READ (IN, 1230) RESID (IRES)

WRITE (IOUT, 1310)

1310 FORMAT (36H ENTER THE RESERVOIR NAME (10 COLS) )

READ (IN, 1320) RESNAM(IRES)

1320 FORMAT (A8)

WRITE (IOUT, 1330)

1330 FORMAT (30H ENTER THE CAPACITY (10 COLS) )

READ (IN , 1120) CAPMAX (IRES)

WRITE (IOUT, 1340)

1340 FORMAT (37H ENTER THE INACTIVE STORAGE (10 COLS) )

READ (IN, 1120) NACTIV(IRES)

WRITE (IOUT, 1350)

1350 FORMAT (42H ENTER THE MAXIMUM SURFACE AREA (10 COLS) )

READ (IN, 1120) AREAMX (IRES)

WRITE (IOUT, 1360)

1360 FORMAT (44H ENTER THE RESERVOIR X COORDINATE (10 COLS) ) READ (IN, 1120) RSXIOC (IRES)

WRITE (IOUT, 1370)

1370 FORMAT (44H ENTER THE RESERVOIR Y COORDINATE (10 COLS) ) READ (IN, 1120) RSYLOC (IRES)

WRITE (IOUT, 1380)

1380 FORMAT (46H ENTER THE INITIAL STORAGE CONTENTS (10 COLS) ) READ (IN, 1120) RESSTR(IRES)

WRITE (IOUT, 1390)

1390 FORMAT (43H ENTER THE INITIAL CONCENTRATION (10 COLS) )

READ (IN, 1120) RSCONC (IRES)

CALL DETNOD (RSXIOC (IRES), RSYLOC (IRES), NODRES (IRES), ISIDE)

SBSTOR(IRES , 1) = RESSTR(IRES) - NACTIV(IRES)

DO 240 IACCNT $=2,5$

240 SBSTOR (IRES, IACCNT) $=0$.

RSXPLT (IRES) $=-.05$

RSYPLT (IRES) $=.05$

GO TO 200

C

C CHANGE RESERVOIR DATA 
Attachment 8--FORTRAN Source Code of Subroutines

for Water-Supply Operations Simulation--Continued

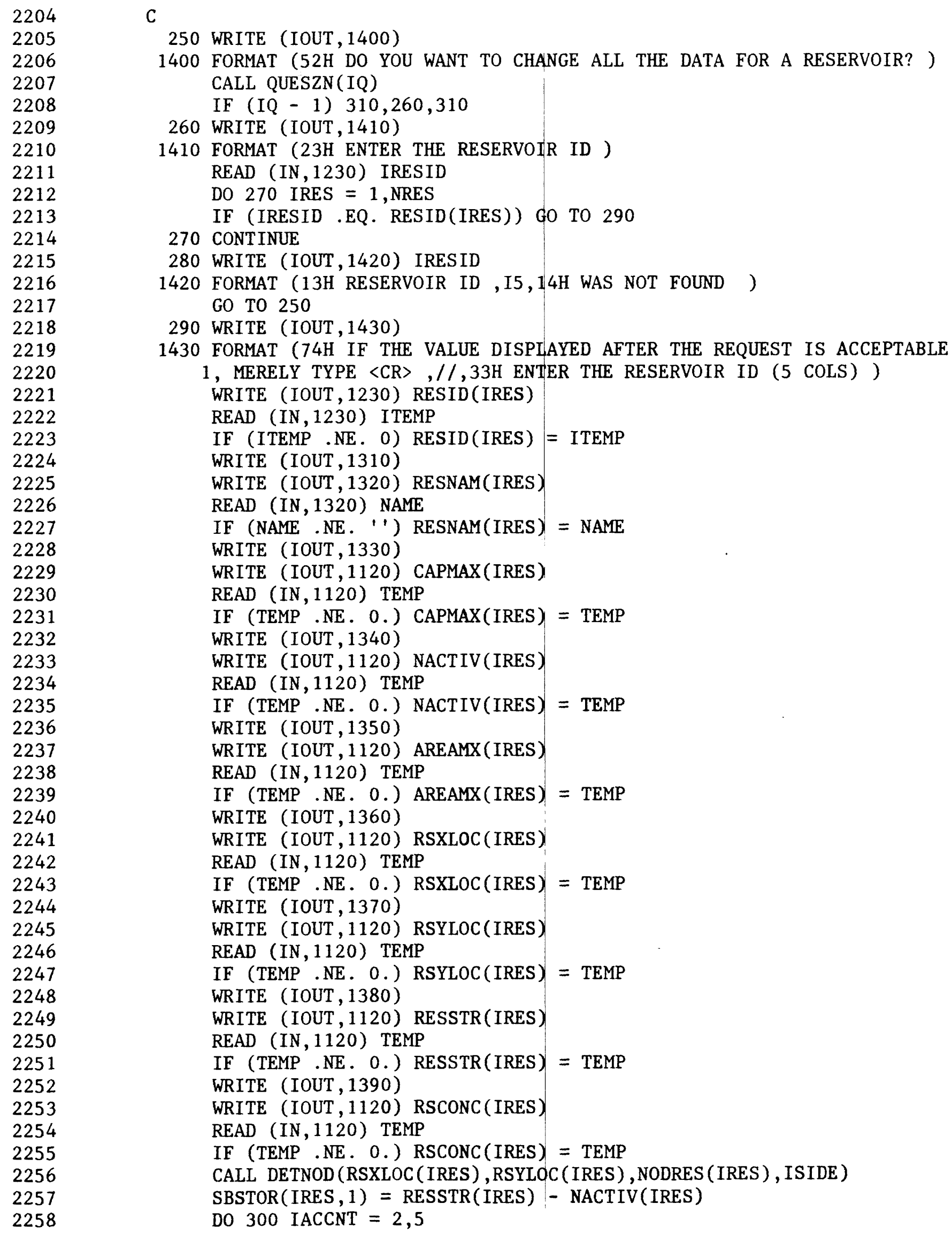

C 250 WRITE (IOUT, 1400)

1400 FORMAT (52H DO YOU WANT TO CHANGE ALL THE DATA FOR A RESERVOIR? ) CALL QUESZN(IQ) IF (IQ - 1) $310,260,310$

260 WRITE (IOUT, 1410)

1410 FORMAT (23H ENTER THE RESERVOIR ID ) READ (IN, 1230) IRESID DO 270 IRES $=1$, NRES IF (IRESID .EQ. RESID(IRES)) GO TO 290

270 CONTINUE

280 WRITE (IOUT, 1420) IRES ID

1420 FORMAT (13H RESERVOIR ID , I5, 14H WAS NOT FOUND ) GO TO 250

290 WRITE (IOUT, 1430)

1430 FORMAT (74H IF THE VALUE DISPLAYED AFTER THE REQUEST IS ACCEPTABLE 1, MERELY TYPE <CR> ,//,33H ENTER THE RESERVOIR ID (5 COLS) ) WRITE (IOUT, 1230) RESID(IRES)

READ (IN, 1230) ITEMP

IF (ITEMP .NE. 0) RESID (IRES) = ITEMP WRITE (IOUT , 1310)

WRITE (IOUT, 1320) RESNAM(IRES)

READ (IN, 1320) NAME

IF (NAME . NE. ' ') RESNAM (IRES) = NAME

WRITE (IOUT, 1330)

WRITE (IOUT, 1120) CAPMAX(IRES)

READ (IN, 1120) TEMP

IF (TEMP . NE. 0.) CAPMAX (IRES) = TEMP

WRITE (IOUT , 1340)

WRITE (IOUT, 1120) NACTIV(IRES)

READ (IN, 1120) TEMP

IF (TEMP .NE. 0.) NACTIV(IRES) = TEMP

WRITE (IOUT, 1350)

WRITE (IOUT, 1120) AREAMX (IRES)

READ (IN, 1120) TEMP

IF (TEMP .NE. 0.) AREAMX (IRES) = TEMP

WRITE (IOUT, 1360)

WRITE (IOUT, 1120) RSXLOC(IRES)

READ (IN, 1120) TEMP

IF (TEMP . NE. O.) RSXLOC (IRES) = TEMP

WRITE (IOUT, 1370)

WRITE (IOUT, 1120) RSYLOC(IRES)

READ (IN, 1120) TEMP

IF (TEMP .NE. O.) RSYLOC (IRES) = TEMP

WRITE (IOUT, 1380)

WRITE (IOUT , 1120) RESSTR (IRES)

READ (IN, 1120) TEMP

IF (TEMP .NE . 0.) RESSTR (IRES) = TEMP

WRITE (IOUT, 1390)

WRITE (IOUT, 1120) RSCONC(IRES)

READ (IN, 1120) TEMP

IF (TEMP .NE. O.) RSCONC (IRES) = TEMP

CALL DETNOD (RSXLOC (IRES), RSYLOC (IRES), NODRES (IRES), ISIDE)

$\operatorname{SBSTOR}($ IRES, 1$)=\operatorname{RESSTR}($ IRES $)-\operatorname{NACTIV}($ IRES $)$

DO 300 IACCNT $=2,5$ 
Attachment 8--FORTRAN Source Code of Subroutines

for Water-Supply Operations Simulation--Continued

2259

2260

2261

2262

2263

2264

2265

2266

2267

2268

2269

2270

2271

2272

2273

2274

2275

2276

2277

2278

2279

2280

2281

2282

2283

2284

2285

2286

2287

2288

2289

2290

2291

2292

2293

2294

2295

2296

2297

2298

2299

2300

2301

2302

2303

2304

2305

2306

2307

2308

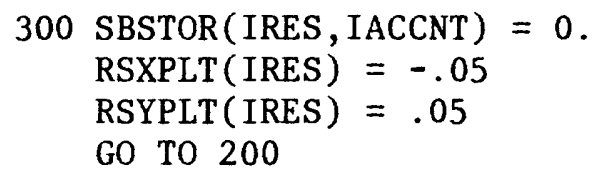

300 SBSTOR (IRES, IACCNT) $=0$.

$\operatorname{RSXPLT}($ IRES) $=-.05$

RSYPLT (IRES) $=.05$

GO TO 200

C

310 WRITE (IOUT, 1440)

1440 FORMAT (61H DO YOU WANT TO CHANGE THE INITIAL CONDITIONS OF A RESE 1RVOIR? )

CALL QUESZN(IQ)

IF (IQ - 1) $360,320,360$

320 WRITE (IOUT, 1410)

READ (IN, 1230) IRESID

DO 330 IRES $=1$, NRES

IF (IRESID .EQ. RESID(IRES)) GO TO 340

330 CONTINUE

GO TO 280

340 WRITE (IOUT, 1380)

READ (IN, 1120) RESSTR(IRES)

$\operatorname{SBSTOR}($ IRES, 1$)=\operatorname{RESSTR}($ IRES $)-\operatorname{NACTIV}($ IRES $)$

DO 350 IACCNT $=2,5$

$350 \operatorname{SBSTOR}$ (IRES, IACCNT) $=0$.

WRITE (IOUT, 1390)

READ (IN, 1120) RSCONC (IRES)

GO TO 200

C

C

C

360 WRITE (IOUT, 1450)

1450 FORMAT (54H DO YOU WANT TO PLOT THE RESERVOIR LOCATION SCHEMATIC?) CALL QUESZN (IQ)

IF (IQ- 1$) 380,370,380$

370 WRITE (IDUM, 1460) TITL

1460 FORMAT (5A4,23H - RESERVOIR LOCATIONS\$)

CALL PLTRES (ITITLE)

C

$\mathrm{C}$

$\mathrm{C}$

380 WRITE $(52,1250)$ NRES

DO 390 IRES $=1$, NRES

WRITE $(52,1270)$ IRES, RESID (IRES), RESNAM (IRES), CAPMAX (IRES),

1 NACTIV(IRES), AREAMX (IRES), RSXLOC (IRES), RSYLOC (IRES), NODRES (IRES),

2 RESSTR(IRES), RSCONC (IRES)

390 CONTINUE

C

C COMPUTE RIVER MILES

C

400 DO 410 INODE $=1$, NNODE

410 RMILES (INODE) $=0$.

INODE = NNODE

RMILES (INODE) $=.00001$ 
Attachment 8--FORTRAN Source Gode of Subroutines

for Water-Supply Operations Simulation--Continued

2309

2310

2311

2312

2313

2314

2315

2316

2317

2318

2319

2320

2321

2322

2323

2324

2325

2326

2327

2328

2329

2330

2331

2332

2333

2334

2335

2336

2337

2338

2339

2340

2341

2342

2343

2344

2345

2346

2347

2348

2349

2350

2351

2352

2353

2354

2355

2356

2357

2358

2359

2360

420 JNODE $=$ NODEUS (INODE)

IF (JNODE .EQ. 0) GO TO 430

SNUOSY $=$ SNUOS 1

IF $($ XLOC $($ NNODE $)$.GT. SNLAT 1$)$ SNUOSY $=$ SNUOS 2

IF (XLOC (JNODE) . GT. SNLAT2) SNUOSY = SNUOS3

RMILES (JNODE) = RMILES (INODE) +

$1 \mathrm{SQRT}(((\mathrm{XLOC}$ (JNODE) -XIOC (INODE) )*CVLONG)**2+((YLOC (JNODE) -

2 YLOC (INODE) ) $*$ CNVLAT) $* 22) *$ SNUOSY

INODE $=$ JNODE

GO TO 420

430 IZERO $=0$

NNOD1 $=$ NNODE -1

DO 440 INODE $=1$, NNOD1

IF (RMILES (INODE) .NE. O.) GO TO 440

JNODE = NXTNOD (INODE)

IF (JNODE . LE. 0) GO TO 440

IZERO $=1$

IF (RMILES (JNODE) .EQ. 0.) GO TO 440

SNUOSY $=$ SNUOS 1

IF (XLOC (INODE) .GT. SNLAT1) SNUOSY = SNUOS2

IF (XLOC (INODE) .GT. SNLAT2) SNUOSY $=$ SNUOS3

RMILES (INODE) = RMILES $($ JNODE $)+$

$1 \mathrm{SQRT}(((\mathrm{XLOC}$ (INODE) -XLOC (JNODE) $) \div$ CVLONG) $*-2+(($ YLOC (INODE) -

2 YLOC (JNODE) $) *$ CNVLAT) $* * 2) \div$ SNUOSY

440 CONTINUE

IF (IZERO .EQ. 1) GO TO 430

C

$\mathrm{C}$

C

WRITE (IOUT, 1470)

1470 FORMAT (61H DO YOU WANT TO DISPLAY THE GROUND-WATER AND RIVER-MILE

1 DATA? )

CALL QUESZN(IQ)

IF (IQ .EQ. 1) WRITE (IOUT, 1480)

WRITE $(52,1480)$

1480 FORMAT (68H1 NODE NODE NODE INITIAL GROUND- INITIAL GROU 1ND- RIVER,/,68H NUMBER ID NAME WATER STORAGE WATER 2CONCENTRATION LENGTH, / , 27X, 42H(ACRE-FEET) (MILLIGRAMS

3 (MILES),$/, 47 \mathrm{X}$, 10HPER LITER) ,/, 25X,33HSIDE 1 SIDE 2 SIDE 1

4 SIDE 2 )

DO 450 INODE $=1$, NNODE

READ $(53,1160)$ (GWSTOR (INODE, IS IDE), GWCONC (INODE, IS IDE), ISIDE=1 ,2)

WRITE $(52,1490)$ INODE, NODE ID (INODE), NODNAM (INODE), GWSTOR (INODE , 1),

1 GWSTOR (INODE , 2), GWCONC (INODE, 1), GWCONC (INODE , 2), RMILES (INODE)

1490 FORMAT ( I5 , I8, 2X,A8, 2F8.0,F9.0,F10.0,F9.1)

IF (IQ .NE. 1) GO TO 450

WRITE (IOUT , 1490) INODE, NODE ID (INODE), NODNAM (INODE),

1 GWSTOR(INODE , 1), GWSTOR (INODE, 2), GWCONC (INODE , 1), GWCONC (INODE , 2),

2 RMILES (INODE)

C

450 CONTINUE

C MODIFY GROUND-WATER DATA 
Attachment 8--FORTRAN Source Code of Subroutines

for Water-Supply Operations Simulation--Continued

2361

2362

2363

2364

2365

2366

2367

2368

2369

2370

2371

2372

2373

2374

2375

2376

2377

2378

2379

2380

2381

2382

2383

2384

2385

2386

2387

2388

2389

2390

2391

2392

2393

2394

2395

2396

2397

2398

2399

2400

2401

2402

2403

2404

2405

2406

2407

2408

2409

2410

2411

2412
C

460 WRITE (IOUT, 1500)

1500 FORMAT (61H DO YOU WANT TO CHANGE ANY OF THE GROUNDWATER INITIAL V 1ALUES? )

CALL QUESZN(IQ)

IF (IQ .EQ. 0) GO TO 500

470 WRITE (IOUT, 1510)

1510 FORMAT (7OH ENTER THE NODE ID FOR THE REACH (5 COLUMNS), AND THE S

1 IDE (5 COLUMNS) )

READ (IN , 1230) NODID, ISIDE

DO 480 INODE $=1$, NNODE

IF (NODID .EQ. NODEID(INODE)) GO TO 490

480 CONTINUE

GO TO 470

490 WRITE (IOUT, 1520)

1520 FORMAT (79H ENTER THE INITIAL STORAGE (10 COLUMNS), AND INITIAL CO INCENTRATION (10 COLUMNS) )

READ (IN , 1120) GWSTOR (INODE , IS IDE) , GWCONC (INODE , ISIDE)

GO TO 460

$\mathrm{C}$

$\mathrm{C}$

C

500 WRITE (IOUT, 1530)

1530 FORMAT (68H ENTER THE FREQUENCY OF PLOTTING THE GROUND-WATER SCHEM 1ATIC (5 COLS) ,/,7X,18H0 FOR NO SCHEMATIC,/,7X,15H12 FOR ANNUALLY,/ $27 \mathrm{X}, 15 \mathrm{H} 99999$ FOR FINAL )

READ (IN, 1540) ISKMGW

1540 FORMAT (16I5)

C

C ENTER NODES AND SIDE FOR GROUND-WATER HYDROGRAPHS

C

WRITE (IOUT, 1550)

1550 FORMAT (62H ENTER THE NODE ID AND SIDE (2=NORTH OR EAST, 1=SOUTH 0 $1 \mathrm{R}$ WEST) $, /, 7 \mathrm{X}, 52 \mathrm{HFOR}$ NODES AT WHICH YOU WANT GROUND-WATER HYDROGRA 2PHS ,/,10X,44H:-2NOTE: 99999 WILL PROVIDE BASINWIDE TOTAL ,/, $37 \mathrm{X}, 32 \mathrm{H}(5$ COLS EACH - LIMIT OF 5 NODES) )

$\operatorname{READ}(\operatorname{IN}, 1540)(\operatorname{IHYGW}(\mathrm{J}), \operatorname{ISID}(\mathrm{J}), \mathrm{J}=1,5)$

DO $540 \mathrm{I}=1,5$

IF (IHYGW(I) .EQ. 99999) GO TO 520

DO $510 \mathrm{~J}=1$, NNODE

IF (IHYGW(I) .NE. NODE ID(J)) GO TO 510

$\operatorname{IHYGW}(I)=\mathrm{J}+1000 * \operatorname{ISID}(\mathrm{I})$

GO TO 540

510 CONTINUE

GO TO 540

520 DO $530 \mathrm{~J}=1,500$

$\operatorname{GWHYS}(J, I)=0$.

$\operatorname{GWHYC}(J, I)=0$.

$530 \operatorname{GWHYRF}(\mathrm{J}, \mathrm{I})=0$.

540 CONTINUE

C

C ENTER NODES AND SIDE FOR MONTHLY GROUND-WATER TABLES 
C WRITE (IOUT, 1560)

1560 FORMAT (62H ENTER THE NODE ID AND SIDE (2=NORTH OR EAST, 1=SOUTH 0 1R WEST) $, /, 7 X, 57$ HFOR NODES AT WHICH YOU WANT TABLES OF GROUND-WATE 2R VALUES,/,10X,44H*:NOTE*:- 99999 WILL PROVIDE BASINWIDE TOTAL ,/, $37 \mathrm{X}, 32 \mathrm{H}$ (5 COLS EACH - LIMIT OF 5 NODES) )

$\operatorname{READ}(\operatorname{IN}, 1540)(\operatorname{ITBLG}(\mathrm{J}), \operatorname{ISID}(\mathrm{J}), \mathrm{J}=1,5)$

DO $580 \quad I=1,5$

IF (ITBLG(I) .EQ. 99999) GO TO 560

DO $550 \mathrm{~J}=1$, NNODE

IF (ITBLG(I) .NE. NODEID(J)) GO TO 550

$\operatorname{ITBLG}(\mathrm{I})=\mathrm{J}+1000 * \operatorname{ISID}(\mathrm{I})$

GO TO 580

550 CONTINUE

GO TO 580

560 DO $570 \mathrm{~J}=1,600$

$\operatorname{IGTBLS}(\mathrm{I}, \mathrm{J})=0$

$\operatorname{IGTBLC}(\mathrm{I}, \mathrm{J})=0$

$570 \operatorname{IGTBLR}(\mathrm{I}, \mathrm{J})=0$

580 CONTINUE

C

C

RETURN

END 
Attachment 8--FORTRAN Source Code of Subroutines

for Water-Supply Operations Simulation--Continued

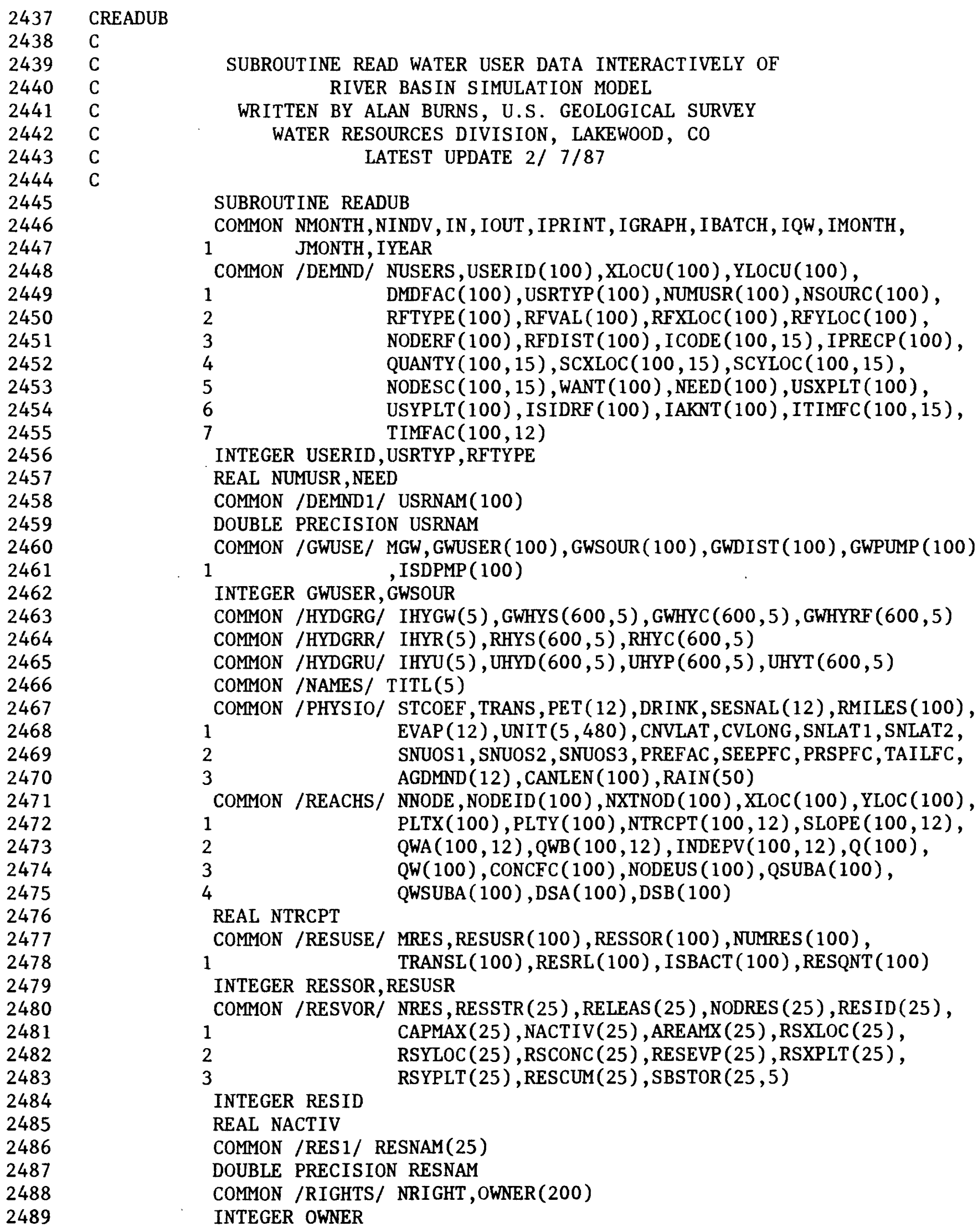


Attachment 8--FORTRAN Source Code of Subroutines

for Water-Supply Operations Simulation--Continued

2490

2491

2492

2493

2494

2495

2496

2497

2498

2499

2500

2501

2502

2503

2504

2505

2506

2507

2508

2509

2510

2511

2512

2513

2514

2515

2516

2517

2518

2519

2520

2521

2522

2523

2524

2525

2526

2527

2528

2529

2530

2531

2532

2533

2534

2535

2536

2537

2538

2539

2540

COMMON /SKMTKU/ ISKMGW, ISKMU,UMAX,ADD (100), AGW(100), ARR (100), $1 \quad \operatorname{AIM}(100), \operatorname{APR}(100), \operatorname{ACN}(100), \operatorname{ANEED}(100)$

COMMON /STATR/ ISSR (10), RMEANS $(10,12), \operatorname{RMEANC}(10,12), \operatorname{RMEANR}(10,12)$, $1 \quad \operatorname{RMEAND}(10,12), \operatorname{RMEANI}(10,12), \operatorname{RMEANE}(10,12)$

COMMON /STATU/ ISSU(10), UMENND $(10,12), \operatorname{UMENDD}(10,12), \operatorname{UMENGW}(10,12)$, $1 \quad \operatorname{UMENRR}(10,12), \operatorname{UMENTA}(10,12), \operatorname{UMENP}(10,12)$,

$2 \operatorname{UMENWC}(10,12), \operatorname{UMENC}(10,12), \operatorname{UMENCL}(10,12)$,

$3 \quad \operatorname{UMENTW}(10,12), \operatorname{UMENDP}(10,12)$

COMMON /TABLEG/ ITBLG(5), IGTBLS $(5,600), \operatorname{IGTBLC}(5,600), \operatorname{IGTBLR}(5,600)$

COMMON /TABLER/ ITBLR(5), IRTBLS $(5,600), \operatorname{IRTBLC}(5,600)$

COMMON /TABLEU/ ITBLU(5), IUTBLD $(5,600), \operatorname{IUTBLP}(5,600), \operatorname{IUTBLT}(5,600)$

$\mathrm{C}$

DIMENSION TITLEU(20), TITLER(20), ITITLE (20)

$\operatorname{INTEGER} * 2$ USED $(100,15)$

CHARACTER $* 80$ IDUM

$\mathrm{C}$

REAL ITITLE

EQUIVALENCE (IDUM, ITITLE)

DOUBLE PRECISION NAME, DATE

$\mathrm{C}$

C

C

READ AND WRITE WATER USER DATA

READ $(50,1000)$ TITLEU

1000 FORMAT (20A4)

READ $(50,1010)$ NUSERS

1010 FORMAT (I5)

WRITE $(52,1020)$ TITLEU, NUSERS

1020 FORMAT (18H1WATER USER FILE: ,20A4,11X,11H, CONTAINS , I3,6H USERS, $1 / / / /)$

$\mathrm{MGW}=0$

MRES $=0$

C

$\mathrm{C}$

C

C

C

C

C

C

C

\section{THERE ARE 5 TYPES OF WATER USERS}

1 = AGRICULTURAL, NUMUSR IS IN IRRIGATED ACRES

2 = MUNCIPAL, NUMUSR IS IN POPULATION

3 = INDUSTRIAL, NUMUSR IS IN UNITS PRODUCED

4 = IN STREAM, NUMUSR IS IN (FISH, SWIMMERS, KILOWATTS ?

5 = RESERVOIR OPERATORS

WRITE $(52,1030)$

1030 FORMAT (55H USER USER USER USER AMOUNT DE- $\quad X \quad$ Y RAIN $1,16 \mathrm{X}, 11 \mathrm{HRETURN}$ FLOW ,28X,6HSOURCE ,/,

2 55H NUM- ID NAME TYPE MAND COORD COORD GAGE, 1X,

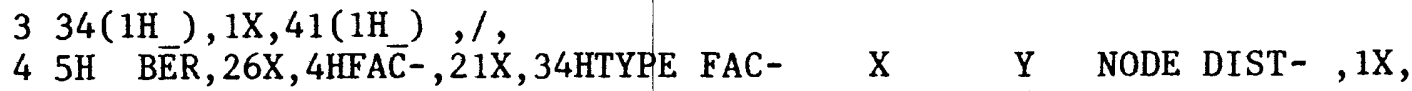

5 41HTYPE LIMIT $X$ Y NODE RES DIST- ,/,

$632 \mathrm{X}, 3 \mathrm{HTOR}, 26 \mathrm{X}, 28 \mathrm{HTOR}$ COORD COORD ANCE , 16X,

7 26HCOORD COORD ID ANCE )

NTIMFC $=0$

DO 160 IUSER $=1$, NUSERS 
Attachment 8--FORTRAN Source Code of Subroutines

for Water-Supply Operations Simulation--Continued

2541

2542

2543

2544

2545

2546

2547

2548

2549

2550

2551

2552

2553

2554

2555

2556

2557

2558

2559

2560

2561

2562

2563

2564

2565

2566

2567

2568

2569

2570

2571

2572

2573

2574

2575

2576

2577

2578

2579

2580

2581

2582

2583

2584

2585

2586

2587

2588

2589

2590

2591

READ $(50,1040)$ USERID(IUSER), USRNAM (IUSER), USRTYP (IUSER),

1 NUMUSR (IUSER), DMDFAC (IUSER), XLOCU (IUSER), YLOCU (IUSER),

2 USXPLT (IUSER), USYPLT (IUSER), IPRECP (IUSER), IAKNT (IUSER),

3 NSOURC (IUSER), RFTYPE (IUSER), RFVAL (IUSER), RFXLOC (IUSER),

4 RFYLOC (IUSER), RFDST

1040 FORMAT (I5 , A8, 2X, I5 , 4F 10.0 , 2F5 .0 , 2I5 ,/ , 15X, 2I5 , 4F 10.0)

CALL DETNOD (RFXLOC (IUSER), RFYLOC (IUSER), INODE, ISIDE)

NODERF (IUSER) = INODE

ISIDRF (IUSER) = ISIDE

CALL CMPDST (INODE, RFXLOC (IUSER), RFYLOC (IUSER), RFDIST (IUSER))

IF (RFDST .NE. O.) RFDIST (IUSER) = RFDST

IF (USRTYP(IUSER) .EQ. 5) GO TO 10

WRITE $(52,1050)$ IUSER, USERID (IUSER), USRNAM (IUSER), USRTYP (IUSER),

1 NUMUSR (IUSER), DMDFAC (IUSER), XLOCU (IUSER), YLOCU (IUSER),

2 IPRECP(IUSER), RFTYPE (IUSER), RFVAL (IUSER), RFXLOC (IUSER),

3 RFYLOC (IUSER), NODERF (IUSER), RFDIST (IUSER), NSOURC (IUSER)

1050 FORMAT $(\mathrm{I} 4, \mathrm{I} 6, \mathrm{IX}, \mathrm{A} 8, \mathrm{I} 3, \mathrm{~F} 8.0, \mathrm{~F} 5.2$, F8 .3 , F7 .3 , $2 \mathrm{I} 4, \mathrm{~F} 6.2$, F8 . 3 , F7 . 3 , I3,

$1 \mathrm{~F} 8.0, \mathrm{I} 2)$

GO TO 40

10 DO 20 IRES $=1$, NRES

IF (RSXLOC (IRES) .EQ. XLOCU(IUSER) .AND. RSYLOC(IRES) .EQ.

1 YLOCU(IUSER)) GO TO 30

20 CONTINUE

WRITE (IOUT, 1060)

1060 FORMAT (76H COORDINATES OF A RESERVOIR OPERATOR DO NOT MATCH COORD

1INATES OF A RESERVOIR )

STOP

30 IRESID = RESID (IRES)

IF (IAKNT(IUSER) .EQ. 0) IAKNT (IUSER) $=1$

WRITE $(52,1070)$ IUSER, USERID (IUSER), USRNAM (IUSER), USRTYP (IUSER),

1 NUMUSR (IUSER), DMDFAC (IUSER), XLOCU(IUSER), YLOCU(IUSER),

2 IAKNT (IUSER), RFTYPE (IUSER), RFVAL (IUSER), RFXLOC (IUSER),

3 RFYLOC (IUSER), NODERF (IUSER), RFDIST(IUSER), NSOURC (IUSER), IRESID

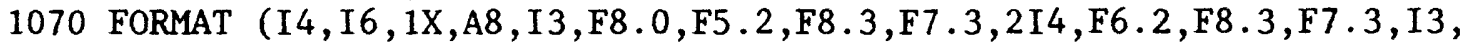

$1 \mathrm{~F} 8.0, \mathrm{I} 2,29 \mathrm{X}, \mathrm{I} 4)$

USRTYP(IUSER) = IRESID

40 NSORC $=$ NSOURC (IUSER)

C

C

C

C

C

$\mathrm{C}$

$\mathrm{C}$

C

C

$\mathrm{C}$

DO 150 ISOURC $=1$, NSORC

READ $(50,1080)$ ICODE (IUSER, ISOURC), QUANTY (IUSER, ISOURC),

1 SCXLOC (IUSER, ISOURC), SCYLOC (IUSER, ISOURC), GWDST , NMRES , IACCNT,

2 ITIMFC (IUSER, ISOURC) 
Attachment 8--FORTRAN Source Code of Subroutines

for Water-Supply Operations Simulation--Continued

2592

2593

2594

2595

2596

2597

2598

2599

2600

2601

2602

2603

2604

2605

2606

2607

2608

2609

2610

2611

2612

2613

2614

2615

2616

2617

2618

2619

2620

2621

2622

2623

2624

2625

2626

2627

2628

2629

2630

2631

2632

2633

2634

2635

2636

2637

2638

2639

2640

2641

2642

2643

2644

1080 FORMAT $(I 5,4 \mathrm{~F} 10.0,3 \mathrm{I} 5)$

IF (ITIMFC (IUSER, ISOURC) .EQ. 0) GO TO 50

NTIMFC $=$ NTIMFC +1

ITIMFC (IUSER, ISOURC) = NTIMFC

READ $(50,1090)$ (TIMFAC (NTIMFC, KMONTH), KMONTH=1, 12)

1090 FORMAT $(5 X, 12 \mathrm{~F} 5.0)$

50 CALL DETNOD (SCXLOC (IUSER, ISOURC), SCYLOC (IUSER, ISOURC),

1 NODESC (IUSER, ISOURC), IS IDE)

IF (ICODE (IUSER, ISOURC) .NE. 1) GO TO 60

SNUOSY $=$ SNUOS 1

IF (XLOCU(IUSER) .GT. SNLAT1) SNUOSY $=$ SNUOS2

IF (XLOCU(IUSER) .GT. SNLAT2) SNUOSY $=$ SNUOS 3

CANLEN (IUSER) =

1 SQRT (( (SCXLOC (IUSER, ISOURC) -XIOCU (IUSER)) *CVLONG) **2

2 ((SCYLOC (IUSER, ISOURC)-YLOCU (IUSER)) *CNVLAT) **2) *SNUOSY

C

60 IF (ICODE (IUSER, ISOURC) .NE. 2) GO TO 70

C FOR GROUND-WATER SOURCES

C

$M G W=M G W+1$

GWUSER(MGW) = IUSER

GWSOUR (MGW) = ISOURC

INODE = NODESC (IUSER, ISOURC)

CALL CMPDST (INODE, SCXLOC (IUSER, ISOURC), SCYLOC (IUSER, ISOURC),

1 GWDIST (MGW) )

IF (GWDST .NE. 0.) GWDIST (MGW) = GWDST

$\operatorname{ISDPMP}(M G W)=$ ISIDE

GO TO 130

C

70 IF (IABS (ICODE (IUSER, ISOURC)) .NE . 3) GO TO 140

$\mathrm{C}$

$\mathrm{C}$

C

80 MRES $=$ MRES +1

RESUSR (MRES) $=$ IUSER

RESSOR (MRES) $=$ ISOURC

C

DO 90 JRES $=1$, NRES

IF (NMRES .EQ. RESID(JRES)) GO TO 100

90 CONTINUE

STOP

C

100 NUMRES (MRES) $=$ JRES

IF (IACCNT .EQ. 0) IACCNT $=1$

ISBACT (MRES) = IACCNT

C

C

C

IF RIVER TRANSMITS RELEASES, COMPUTE TRANSIT LOSS

IF (ICODE (IUSER, ISOURC) .LT. 0) GO TO 110

JNODE $=$ NODRES (JRES)

KNODE $=$ NODESC (IUSER, ISOURC)

TRANSL (MRES) $=1 .-.0007 \div$ ABS (RMILES (JNODE) $-\operatorname{RMILES}($ KNODE) $)$

GO TO 120 
Attachment 8--FORTRAN Source Code of Subroutines

for Water-Supply Operations Simulation--Continued

2645

2646

2647

2648

2649

2650

2651

2652

2653

2654

2655

2656

2657

2658

2659

2660

2661

2662

2663

2664

2665

2666

2667

2668

2669

2670

2671

2672

2673

2674

2675

2676

2677

2678

2679

2680

2681

2682

2683

2684

2685

2686

2687

2688

2689

2690

2691

2692

2693

2694

2695

2696
$\mathrm{C}$

C

C

110 NODESC (IUSER, ISOURC) $=$ NNODE +1

ICODE (IUSER, ISOURC) $=-$ ICODE (IUSER, ISOURC)

TRANSL (MRES) $=1$.

$\mathrm{C}$

120 WRITE $(52,1100)$ ICODE (IUSER, ISOURC), QUANTY (IUSER, ISOURC),

1 SCXLOC (IUSER, ISOURC), SCYLOC (IUSER, ISOURC), NODESC (IUSER, ISOURC), 2 NUMRES (MRES)

1100 FORMAT (90X,I3,F9.1,F8.3,F7.3,2I4)

GO TO 150

130 WRITE $(52,1110)$ ICODE (IUSER, ISOURC), QUANTY (IUSER, ISOURC),

1 SCXLOC (IUSER, ISOURC), SCYLOC (IUSER, ISOURC), NODESC (IUSER, ISOURC),

2 GWDIST (MGW)

1110 FORMAT $(90 \mathrm{X}, \mathrm{I} 3, \mathrm{~F} 9.1, \mathrm{~F} 8.3, \mathrm{~F} 7.3, \mathrm{I} 4,4 \mathrm{X}, \mathrm{F} 7.0)$

GO TO 150

C

140 IF (ICODE (IUSER, ISOURC) .EQ. 6) GO TO 80

WRITE $(52,1100)$ ICODE (IUSER, ISOURC), QUANTY (IUSER, ISOURC),

1 SCXLOC (IUSER, ISOURC), SCYLOC (IUSER, ISOURC), NODESC (IUSER, ISOURC)

150 CONTINUE

160 CONTINUE

C

$\mathrm{C}$

C

CALL QUESZN(IQ)

IF (IQ- 1 ) $180,170,180$

170 WRITE (IDUM, 1120) TITL

1120 FORMAT (5A4,24H - WATER USER LOCATIONS\$)

CALL PLTUSR(ITITLE)

C

C READ, WRITE, AND CHECK WATER RIGHT FILE

C

180 READ $(51,1000)$ TITLER

READ $(51,1010)$ NRIGHT

WRITE $(52,1130)$ TITLER, NRIGHT

1130 FORMAT (19H1WATER RIGHT FILE: ,20A4,9X,11H, CONTAINS , I3, 7H RIGHTS

$1, / / /, 50 \mathrm{H}$ OWNER OWNER DATE AMOUNT ,/,

2 ID NAME )

DO 190 IUSER $=1$, NUSERS

DO 190 ISOURC $=1,15$

190 USED (IUSER, ISOURC) $=0$

$\mathrm{C}$

DO 250 IRIGHT $=1$, NRIGHT

READ (51, 1140) OWNER (IRIGHT), NAME, DATE , AMNT

1140 FORMAT (I5, A8, 2X, A8, F10.0)

C

DO 200 IUSER $=1$, NUSERS

IF (OWNER(IRIGHT) .EQ. USERID(IUSER)) GO TO 210

200 CONTINUE

WRITE $(52,1150)$ OWNER(IRIGHT) 
Attachment 8--FORTRAN Source Code of Subroutines for Water-Supply Operations Simulation--Continued

2706

2707

2708

2709

2710

2711

2712

2713

2714

2715

2716

2717

2718

2719

2720

2721

2722

2723

2724

2725

2726

2727

2728

2729

2730

2731

2732

2733

2734

2735

2736

2737

2738

2739

2740

2741

2742

2743

2744

2745

2746

2747

2748

1150 FORMAT (24H THERE IS NO LISTING OF, I5,23H IN THE WATER USER FILE) GO TO 250

210 ISOURC $=0$

220 ISOURC $=$ ISOURC +1

IF (ISOURC .GT. NSOURC(IUSER)) GO TO 240

IF (ICODE (IUSER, ISOURC) .EQ. 1) GO TO 230

IF (ICODE (IUSER, ISOURC) .EQ. 6) GO TO 230

IF (ICODE (IUSER, ISOURC) .NE. 5) GO TO 220

230 IF (USED (IUSER, ISOURC) .EQ. 1) GO TO 220

USED (IUSER, ISOURC) $=1$

GO TO 250

240 WRITE $(52,1160)$ OWNER(IRIGHT), IRIGHT

1160 FORMAT (6H USER , I5,34H DOES NOT HAVE A SOURCE FOR RIGHT ,I3)

250 WRITE $(52,1170)$ OWNER (IRIGHT), NAME, DATE , AMNT

1170 FORMAT ( $5 \mathrm{X}, \mathrm{I} 5,5 \mathrm{X}, \mathrm{A} 8,5 \mathrm{X}, \mathrm{A} 8,2 \mathrm{X}, \mathrm{F} 12.2$ )

$\mathrm{C}$

DO 270 IUSER $=1$, NUSERS

NSOUR = NSOURC (IUSER)

DO 270 ISOURC $=1$, NSOUR

IF (ICODE (IUSER, ISOURC) .EQ. 1) GO TO 260

IF (ICODE (IUSER, ISOURC) .EQ. 6) GO TO 260

IF (ICODE (IUSER, ISOURC) .NE. 5) GO TO 270

260 IF (USED (IUSER, ISOURC) .EQ. 1 .OR. QUANTY(IUSER, ISOURC) .EQ. 0.) 1 GO TO 270

WRITE $(52,1180)$ USERID(IUSER), ISOURC

1180 FORMAT (1OH FOR USER , I5, 13H, ITS SOURCE ,I3,

1 19H HAS NO WATER RIGHT )

270 CONTINUE

C

C ENTER FREQUENCY OF PLOTTING WATER USER SCHEMATIC

$\mathrm{C}$

READ (IN, 1080) ISKMU, UMAX

$\mathrm{C}$

$\mathrm{C}$

$\mathrm{C}$

ENTER WATER USERS FOR HYDROGRAPHS

READ (IN, 1190) IHYU

1190 FORMAT (16I5)

DO $310 \mathrm{I}=1,5$

IF (IHYU(I) .EQ. 99999) GO TO 290

DO $280 \mathrm{~J}=1$, NUSERS

IF (IHYU(I) .NE. USERID (J)) GO TO 280

$\operatorname{IHYU}(\mathrm{I})=\mathrm{J}$

GO TO 310

280 CONTINUE

GO TO 310

290 DO $300 \mathrm{~J}=1,600$

$\operatorname{UHYD}(J, I)=0$.

$\operatorname{UHYP}(J, I)=0$.

$300 \operatorname{UHYT}(\mathrm{J}, \mathrm{I})=0$.

310 CONTINUE

C

C ENTER WATER USERS FOR MONTHLY TABLES 
Attachment 8--FORTRAN Source Code of Subroutines for Water-Supply Operations Simulation--Continued

C

READ (IN , 1190) ITBLU

DO $350 I=1,5$

IF (ITBLU(I) .EQ. 99999) GO TO 330

DO $320 \mathrm{~J}=1$, NUSERS

IF (ITBLU(I) .NE. USERID(J)) GO TO 320

$\operatorname{ITBLU}(\mathrm{I})=\mathrm{J}$

GO TO 350

320 CONTINUE

GO TO 350

330 DO $340 \mathrm{~J}=1,600$

$\operatorname{IUTBLD}(\mathrm{I}, \mathrm{J})=0$

$\operatorname{IUTBLP}(I, J)=0$

$340 \operatorname{IUTBLT}(\mathrm{I}, \mathrm{J})=0$

350 CONTINUE

$\mathrm{C}$

$\mathrm{C}$

C

ENTER WATER USERS FOR STATISTICAL SUMMARIES

READ (IN, 1190) ISSU

DO $380 I=1,10$

IF (ISSU(I) .LE. 0) GO TO 380

DO $360 \mathrm{~J}=1,12$

$\operatorname{UMENND}(I, J)=0$.

$\operatorname{UMENDD}(I, J)=0$.

$\operatorname{UMENGW}(I, J)=0$.

$\operatorname{UMENRR}(I, J)=0$.

$\operatorname{UMENTA}(I, J)=0$.

$\operatorname{UMENP}(I, J)=0$.

$\operatorname{UMENWC}(I, J)=0$.

$\operatorname{UMENC}(I, J)=0$.

$\operatorname{UMENCL}(I, J)=0$.

$\operatorname{UMENTW}(I, J)=0$.

$360 \operatorname{UMENDP}(\mathrm{I}, \mathrm{J})=0$.

DO $370 \mathrm{~J}=1$, NUSERS

IF (ISSU(I) .NE. USERID(J)) GO TO 370

$\operatorname{ISSU}(\mathrm{I})=\mathrm{J}$

GO TO 380

370 CONTINUE

380 CONTINUE

C

C

C

READ (IN, 1190) IHYR

DO $400 \mathrm{I}=1,5$

DO $390 \mathrm{~J}=1$, NRES

IF (IHYR(I) .NE. RESID(J)) GO TO 390

$\operatorname{IHYR}(\mathrm{I})=\mathrm{J}$

GO TO 400

390 CONTINUE

400 CONTINUE

C

C 
Attachment 8--FORTRAN Source Code of Subroutines

for Water-Supply Operations Simulation--Continued

C

READ (IN, 1190) ITBLR

DO $420 \mathrm{I}=1,5$

DO $410 \mathrm{~J}=1$, NRES

IF (ITBLR(I) .NE. RESID(J)) GQ TO 410

$\operatorname{ITBLR}(\mathrm{I})=\mathrm{J}$

GO TO 420

410 CONT INUE

420 CONTINUE

C

C

C

ENTER RESERVOIRS FOR STATISTICAL SUMMARIES

READ (IN, 1190) ISSR

DO $450 \mathrm{I}=1,10$

IF (ISSR(I) .IE. 0) GO TO 450

D0 $430 \mathrm{~J}=1,12$

$\operatorname{RMEANS}(I, J)=0$.

$\operatorname{RMEANC}(I, J)=0$.

$\operatorname{RMEANR}(I, J)=0$.

$\operatorname{RMEAND}(\mathrm{I}, \mathrm{J})=0$.

$\operatorname{RMEANI}(I, J)=0$.

$430 \operatorname{RMEANE}(I, J)=0$.

DO $440 \mathrm{~J}=1$, NRES

IF (ISSR(I) .NE. RESID(J)) GO TO 440

$\operatorname{ISSR}(\mathrm{I})=\mathrm{J}$

GO TO 450

440 CONTINUE

450 CONTINUE

C

RETURN

END 
Attachment 8--FORTRAN Source Code of Subroutines

for Water-Supply Operations Simulation--Continued

\begin{tabular}{|c|c|c|}
\hline $\begin{array}{l}2832 \\
2833\end{array}$ & $\begin{array}{l}\text { CREADUI } \\
\mathrm{C}\end{array}$ & \\
\hline 2834 & $\mathrm{C}$ & SUBROUTINE READ WATER USER DATA INTERACTIVELY OF \\
\hline 2835 & C & RIVER BASIN SIMULATION MODEL \\
\hline 2836 & $\mathrm{C}$ & WRITTEN BY ALAN BURNS, U.S. GEOLOGICAL SURVEY \\
\hline 2837 & C & WATER RESOURCES DIVISION, LAKEWOOD, CO \\
\hline 2838 & $\mathrm{C}$ & LATEST UPDATE $2 / 7 / 87$ \\
\hline 2839 & $\mathrm{C}$ & \\
\hline $2840^{-}$ & & SUBROUTINE READUI \\
\hline 2841 & & COMMON NMONTH, NINDV, IN , IOUT, IPRINT, IGRAPH, IBATCH, IQW, IMONTH, \\
\hline 2842 & & JMONTH, IYEAR \\
\hline 2843 & & COMMON /DEMND/ NUSERS, USERID (100), XIOCU (100), YLOCU (100), \\
\hline 2844 & & DMDFAC $(100), \operatorname{USRTYP}(100), \operatorname{NUMUSR}(100), \operatorname{NSOURC}(100)$, \\
\hline 2845 & & RFTYPE (100), RFVAL (100), RFXIOC (100), RFYLOC $(100)$ \\
\hline 2846 & & NODERF $(100), \operatorname{RFDIST}(100), \operatorname{ICODE}(100,15), \operatorname{IPRECP}(100)$, \\
\hline 2847 & & $\operatorname{QUANTY}(100,15), \operatorname{SCXLOC}(100,15), \operatorname{SCYLOC}(100,15)$ \\
\hline 2848 & & NODESC $(100,15), \operatorname{WANT}(100), \operatorname{NEED}(100), \operatorname{USXPLT}(100)$, \\
\hline 2849 & & USYPLT $(100), \operatorname{ISIDRF}(100), \operatorname{IAKNT}(100), \operatorname{ITIMFC}(100,15)$, \\
\hline 2850 & & TIMFAC $(100,12)$ \\
\hline 2851 & & INTEGER USERID, USRTYP, RFTYPE \\
\hline 2852 & & REAL NUMUSR, NEED \\
\hline 2853 & & COMMON /DEMND1/ USRNAM(100) \\
\hline 2854 & & DOUBLE PRECISION USRNAM \\
\hline 2855 & & COMMON / GWUSE / MGW, GWUSER(100), GWSOUR (100), GWDIST (100), GWPUMP (100) \\
\hline 2856 & & $1 \quad, \operatorname{ISDPMP}(100)$ \\
\hline 2857 & & INTEGER GWUSER, GWSOUR \\
\hline 2858 & & COMMON /HYDGRG/ IHYGW (5), GWHYS $(600,5)$, GWHYC $(600,5)$, GWHYRF $(600,5)$ \\
\hline 2859 & & COMMON /HYDGRR/ IHYR (5), RHYS $(600,5), \operatorname{RHYC}(600,5)$ \\
\hline 2860 & & COMMON /HYDGRU/ IHYU (5), UHYD $(600,5), \operatorname{UHYP}(600,5), \operatorname{UHYT}(600,5)$ \\
\hline 2861 & & COMMON /NAMES/ TITL(5) \\
\hline 2862 & & COMMON /PHYSIO/ STCOEF, TRANS, PET(12),DRINK, SESNAL(12), RMILES (100), \\
\hline 2863 & & $1 \quad \operatorname{EVAP}(12), \operatorname{UNIT}(5,480)$, CNVLAT , CVLONG, SNLAT 1 , SNLAT2, \\
\hline 2864 & & SNUOS1, SNUOS2, SNUOS3, PREFAC, SEEPFC, PRSPFC, TAILFC, \\
\hline 2865 & & $\operatorname{AGDMND}(12), \operatorname{CANLEN}(100), \operatorname{RAIN}(50)$ \\
\hline 2866 & & COMMON /REACHS/ NNODE, NODEID (100), NXTNOD $(100), \operatorname{XLOC~}(100)$, YLOC $(100)$, \\
\hline 2867 & & $\operatorname{PLTX}(100), \operatorname{PLTY}(100), \operatorname{NTRCPT}(100,12), \operatorname{SLOPE}(100,12)$ \\
\hline 2868 & & $Q W A(100,12), Q W B(100,12), \operatorname{INDEPV}(100,12), Q(100)$ \\
\hline 2869 & & QW $(100), \operatorname{CONCFC}(100), \operatorname{NODEUS}(100), \mathrm{QSUBA}(100)$ \\
\hline 2870 & & QWSUBA(100),DSA(100),DSB(100) \\
\hline 2871 & & REAL NTRCPT \\
\hline 2872 & & COMMON /RESUSE/ MRES,RESUSR(100), RESSOR(100), NUMRES (100), \\
\hline 2873 & & $1 \quad \operatorname{TRANSL}(100), \operatorname{RESRL}(100), \operatorname{ISBACT}(100), \operatorname{RESQNT}(100)$ \\
\hline 2874 & & INTEGER RESSOR, RESUSR \\
\hline 2875 & & COMMON /RESVOR/ NRES, RESSTR (25), RELEAS (25), NODRES (25), RESID (25), \\
\hline 2876 & & $1 \quad$ CAPMAX (25), NACTIV (25), AREAMX (25), RSXLOC (25), \\
\hline 2877 & & RSYLOC (25), RSCONC (25), RESEVP (25), RSXPLT (25), \\
\hline 2878 & & $\operatorname{RSYPLT}(25), \operatorname{RESCUM}(25), \operatorname{SBSTOR}(25,5)$ \\
\hline 2879 & & INTEGER RESID \\
\hline 2880 & & REAL NACTIV \\
\hline 2881 & & COMMON /RES1/ RESNAM(25) \\
\hline 2882 & & DOUBLE PRECISION RESNAM \\
\hline 2883. & & COMMON /RIGHTS/ NRIGHT, OWNER(20 \\
\hline 2884 & & INTEGER OWNER \\
\hline
\end{tabular}


Attachment 8--FORTRAN Source Code of Subroutines for Water-Supply Operations Simulation--Continued

2885

2886

2887

2888

2889

2890

2891

2892

2893

2894

2895

2896

2897

2898

2899

2900

2901

2902

2903

2904

2905

2906

2907

2908

2909

2910

2911

2912

2913

2914

2915

2916

2917

2918

2919

2920

2921

2922

2923

2924

2925

2926

2927

2928

2929

2930

2931

2932

2933

2934

2935

2936

2937

COMMON /SKMTKU/ ISKMGW, ISKMU, UMAX, ADD (100), AGW(100), ARR (100),

$1 \quad \operatorname{AIM}(100), \operatorname{APR}(100), \operatorname{ACN}(100), \operatorname{ANEED}(100)$

COMMON /STATR/ ISSR (10), RMEANS $(10,12), \operatorname{RMEANC}(10,12), \operatorname{RMEANR}(10,12)$,

$1 \quad \operatorname{RMEAND}(10,12), \operatorname{RMEANI}(10,12), \operatorname{RMEANE}(10,12)$

COMMON /STATU/ ISSU(10), UMENND $(10,12), \operatorname{UMENDD}(10,12), \operatorname{UMENGW}(10,12)$,

$1 \quad \operatorname{UMENRR}(10,12), \operatorname{UMENTA}(10,12), \operatorname{UMENP}(10,12)$,

$2 \quad \operatorname{UMENWC}(10,12), \operatorname{UMENC}(10,12), \operatorname{UMENCL}(10,12)$,

$3 \quad \operatorname{UMENTW}(10,12), \operatorname{UMENDP}(10,12)$

COMMON /TABLEG/ ITBLG(5), IGTBLS $(5,600), \operatorname{IGTBLC}(5,600), \operatorname{IGTBLR}(5,600)$

COMMON /TABLER/ ITBLR(5), IRTBLS $(5,600), \operatorname{IRTBLC}(5,600)$

COMMON /TABLEU/ ITBLU(5), IUTBLD $(5,600), \operatorname{IUTBLP}(5,600), \operatorname{IUTBLT}(5,600)$

C

DIMENSION TITLEU(20), TITLER(20), ITITLE (20), AMNT (200)

INTEGER $* 2$ USED $(100,15)$

CHARACTER $* 80$ IDUM

C

DOUBLE PRECISION NAME (200), DATE (200), NAME 1

REAL ITITLE

EQUIVALENCE (IDUM, ITITLE)

$\mathrm{C}$

$\mathrm{C}$

$\mathrm{C}$

READ AND DISPLAY WATER USER DATA

READ $(50,2000)$ TITLEU

2000 FORMAT (20A4)

READ $(50,2010)$ NUSERS

2010 FORMAT (I5)

WRITE $(52,2020)$ TITLEU, NUSERS

2020 FORMAT (18H1WATER USER FILE: ,20A4,11X,11H, CONTAINS , I3,6H USERS,

$1 / / / /)$

$\mathrm{MGW}=0$

MRES $=0$

C

C

C

C

$\mathrm{C}$

C

C

$\mathrm{C}$

$\mathrm{C}$

THERE ARE 5 TYPES OF WATER USERS

$1=$ AGRICULTURAL, NUMUSR IS IN IRRIGATED ACRES

$2=$ MUNCIPAL, NUMUSR IS IN POPULATION

3 = INDUSTRIAL, NUMUSR IS IN UNITS PRODUCED

4 = IN STREAM, NUMUSR IS IN (FISH, SWIMMERS, KILOWATTS ?

5 = RESERVOIR OPERATORS

WRITE (IOUT, 2030)

2030 FORMAT (38H DO YOU WANT TO DISPLAY THE USER DATA? )

CALL QUESZN(IQ)

IF (IQ .EQ. 1) WRITE (IOUT, 2040)

2040 FORMAT (55H USER USER USER USER AMOUNT DE- $\quad X \quad$ Y RAIN

$1,16 \mathrm{X}, 11 \mathrm{HRETURN}$ FLOW ,28X,6HSOURCE ,/,

$255 \mathrm{H}$ NUM- ID NAME TYPE MAND COORD COORD GAGE , 1X,

$334\left(1 \mathrm{H}_{-}\right), 1 \mathrm{X}, 41\left(1 \mathrm{H}_{-}\right), /$,

$45 \mathrm{H}$ BER, 26X,4HFAC-,21X,34HTYPE FAC- $X \quad Y$ NODE DIST- , 1X,

5 41HTYPE LIMIT $X$ Y NODE RES DIST- ,/,

$632 \mathrm{X}, 3 \mathrm{HTOR}, 26 \mathrm{X}, 28 \mathrm{HTOR}$ COORD COORD ANCE , 16X,

7 26HCOORD COORD ID ANCE )

NTIMFC $=0$ 
Attachment 8--FORTRAN Source Code of Subroutines

for Water-Supply Operations Simulation--Continued

2938

2939

2940

2941

2942

2943

2944

2945

2946

2947

2948

2949

2950

2951

2952

2953

2954

2955

2956

2957

2958

2959

2960

2961

2962

2963

2964

2965

2966

2967

2968

2969

2970

2971

2972

2973

2974

2975

2976

2977

2978

2979

2980

2981

2982

2983

2984

2985

2986

2987

2988
C

DO 170 IUSER $=1$, NUSERS

READ (50,2050) USERID (IUSER), USRNAM (IUSER), USRTYP (IUSER),

1 NUMUSR (IUSER), DMDFAC (IUSER), XLOCU (IUSER), YLOCU (IUSER),

2 USXPLT (IUSER), USYPLT (IUSER), IPRECP (IUSER), IAKNT (IUSER),

3 NSOURC (IUSER), RFTYPE (IUSER), RFVAL (IUSER), RFXLOC (IUSER),

4 RFYLOC (IUSER), RFDST

2050 FORMAT (I5 , A8 , 2X, I5 , 4F 10.0 ,2F5.0, 2I5,/, 15X, 2I5 , 4F 10.0)

CALL DETNOD (RFXLOC (IUSER), RFYLOC (IUSER), INODE, ISIDE)

NODERF (IUSER) = INODE

$\operatorname{ISIDRF}($ IUSER) $=$ ISIDE

CALL CMPDST (INODE, RFXLOC (IUSER), RFYLOC (IUSER), RFDIST (IUSER))

IF (RFDST .NE. 0.) RFDIST (IUSER) $=$ RFDST

IF (USRTYP(IUSER) .EQ. 5) GO TO 10

IF (IQ .NE. 1) GO TO 50

WRITE (IOUT, 2060) IUSER, USERID (IUSER), USRNAM (IUSER), USRTYP (IUSER),

1 NUMUSR (IUSER), DMDFAC (IUSER), XLOCU (IUSER), YLOCU (IUSER),

2 IPRECP (IUSER), RFTYPE (IUSER), RFVAL (IUSER), RFXLOC (IUSER),

3 RFYLOC (IUSER), NODERF (IUSER), RFDIST (IUSER)

2060 FORMAT $(\mathrm{I} 4, \mathrm{I} 6, \mathrm{IX}, \mathrm{A} 8, \mathrm{I} 3, \mathrm{~F} 8.0, \mathrm{~F} 5.2, \mathrm{~F} 8.3, \mathrm{~F} 7.3,2 \mathrm{I} 4, \mathrm{~F} 6.2, \mathrm{~F} 8.3, \mathrm{~F} 7.3$, I3 ,

$1 \mathrm{~F} 8.0, \mathrm{I} 2)$

GO TO 50

C

10 DO 20 IRES $=1$, NRES

IF (RSXLOC (IRES) .EQ. XLOCU(IUSER) .AND. RSYLOC(IRES) .EQ.

1 YLOCU(IUSER)) GO TO 30

20 CONTINUE

WRITE (IOUT, 2070)

2070 FORMAT (76H COORDINATES OF A RESERVOIR OPERATOR DO NOT MATCH COORD 1INATES OF A RESERVOIR ) STOP

30 IRESID = RESID (IRES)

IF (IAKNT (IUSER) .EQ. 0 ) IAKNT (IUSER) $=1$

IF (IQ .NE. 1) GO TO 40

WRITE (IOUT , 2080) IUSER, USERID (IUSER), USRNAM(IUSER), USRTYP (IUSER),

1 NUMUSR (IUSER), DMDFAC (IUSER), XLOCU (IUSER), YLOCU (IUSER),

2 IAKNT (IUSER), RFTYPE (IUSER), RFVAL (IUSER), RFXLOC (IUSER),

3 RFYLOC (IUSER), NODERF (IUSER), RFDIST (IUSER), NSOURC (IUSER), IRES ID

2080 FORMAT (I4, I6, IX, A8, I3 , F8 .0, F5 .2, F8 .3, F7.3, $2 \mathrm{I} 4, \mathrm{~F} 6.2, \mathrm{~F} 8.3, \mathrm{~F} 7.3, \mathrm{I} 3$,

1 F8.0,I2, 29X, I4)

40 USRTYP (IUSER) = IRESID

50 NSORC $=$ NSOURC (IUSER)

C

C

C

C

C.

C

C

C

C
THERE ARE 6 TYPES OF WATER SOURCES

1 = DIRECT DIVERSIONS

2 = GROUND-WATER PUMPAGE

3 = RESERVOIR RELEASES

4 = DIRECT PIPELINE IMPORTATION

5 = STORAGE DIVERSION

6 = DIRECT DIVERSION CHANGED TO STORAGE 
Attachment 8--FORTRAN Source Code of Subroutines

for Water-Supply Operations Simulation--Continued

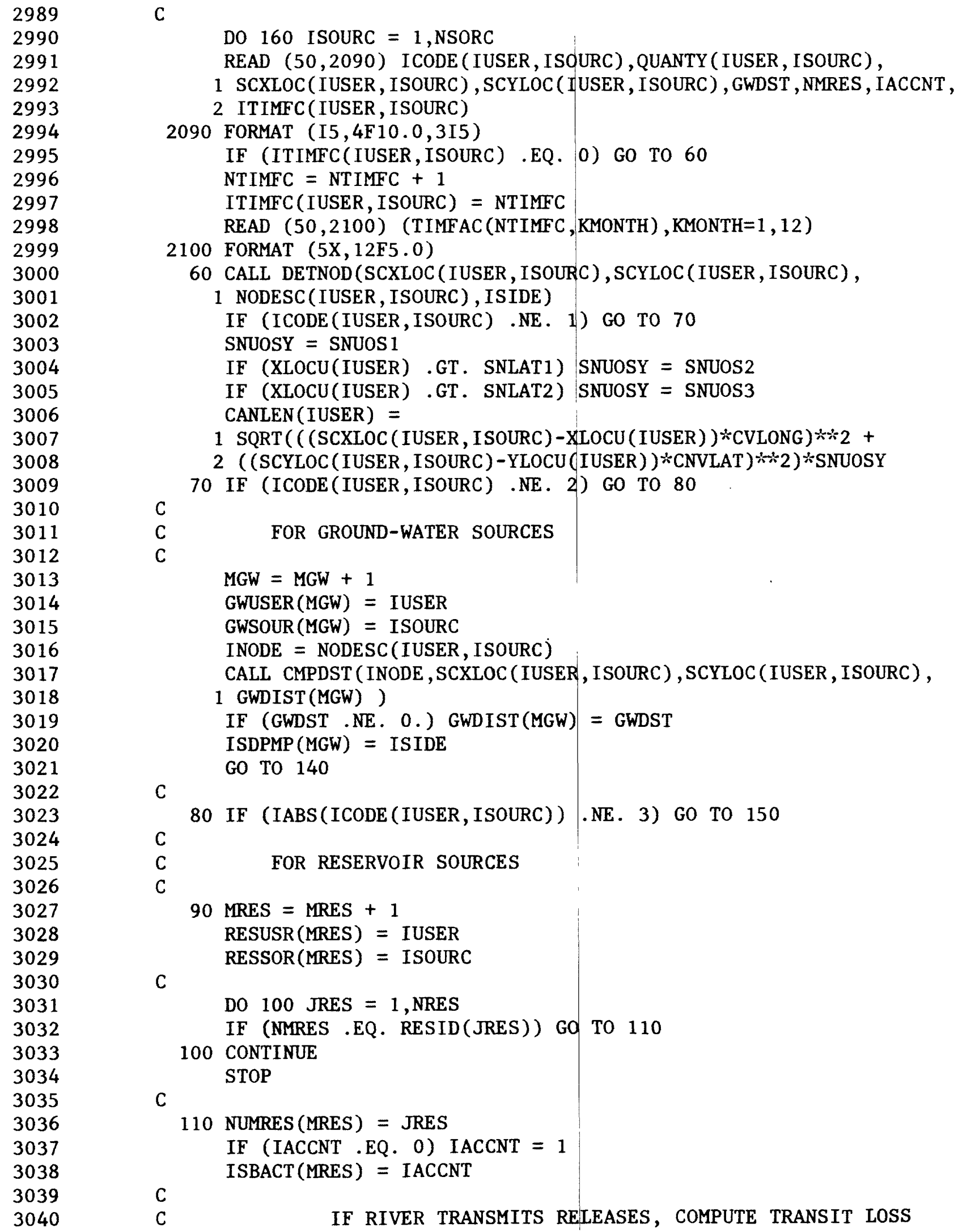

C

DO 160 ISOURC $=1$, NSORC

READ $(50,2090)$ ICODE (IUSER, ISOURC), QUANTY (IUSER, ISOURC),

1 SCXLOC (IUSER, ISOURC), SCYLOC (IUSER, ISOURC), GWDST , NMRES, IACCNT ,

2 ITIMFC (IUSER, ISOURC)

2090 FORMAT (I5, 4F $10.0,3$ I5)

IF (ITIMFC(IUSER, ISOURC) .EQ. 0) GO TO 60

NTIMFC $=$ NTIMFC +1

ITIMFC (IUSER, ISOURC) = NTIMFC

READ $(50,2100)$ (TIMFAC (NTIMFC, KMONTH), KMONTH $=1,12$ )

2100 FORMAT $(5 \mathrm{X}, 12 \mathrm{~F} 5.0)$

60 CALL DETNOD (SCXLOC (IUSER, ISOURC), SCYLOC (IUSER, ISOURC),

1 NODESC (IUSER, ISOURC), ISIDE)

IF (ICODE (IUSER, ISOURC) .NE. 1) GO TO 70

SNUOSY $=$ SNUOS 1

IF (XLOCU(IUSER) .GT. SNLAT1) SNUOSY = SNUOS2

IF (XIOCU(IUSER) .GT. SNLAT2) SNUOSY = SNUOS3

CANLEN (IUSER) $=$

1 SQRT (( (SCXIOC (IUSER, ISOURC) -XLOCU (IUSER)) *CVLONG) $* 2+$

2 ((SCYLOC (IUSER, ISOURC)-YLOCU (IUSER)) *CNVLAT) **2)*SNUOSY

C

C

C

70 IF (ICODE (IUSER, ISOURC) .NE. 2) GO TO 80

FOR GROUND-WATER SOURCES

$M G W=M G W+1$

GWUSER (MGW) = IUSER

GWSOUR (MGW) $=$ ISOURC

INODE = NODESC (IUSER, ISOURC)

CALL CMPDST (INODE, SCXLOC (IUSER, ISOURC), SCYLOC (IUSER, ISOURC), 1 GWDIST (MGW) )

IF (GWDST .NE. 0.) GWDIST (MGW) = GWDST

$\operatorname{ISDPMP}(M G W)=$ ISIDE

GO TO 140

C

80 IF (IABS (ICODE (IUSER, ISOURC)) .NE. 3) GO TO 150

$\mathrm{C}$

$\mathrm{C}$

C

90 MRES $=$ MRES +1

RESUSR (MRES) $=$ IUSER

RESSOR(MRES) $=$ ISOURC

C

DO 100 JRES $=1$, NRES

IF (NMRES .EQ. RESID(JRES)) GO TO 110

100 CONTINUE

STOP

C

110 NUMRES (MRES) = JRES

IF (IACCNT .EQ. 0) IACCNT $=1$

ISBACT (MRES) = IACCNT

C

IF RIVER TRANSMITS RELEASES, COMPUTE TRANSIT LOSS 
Attachment 8--FORTRAN Source Code of Subroutines

for Water-Supply Operations Simulation--Continued

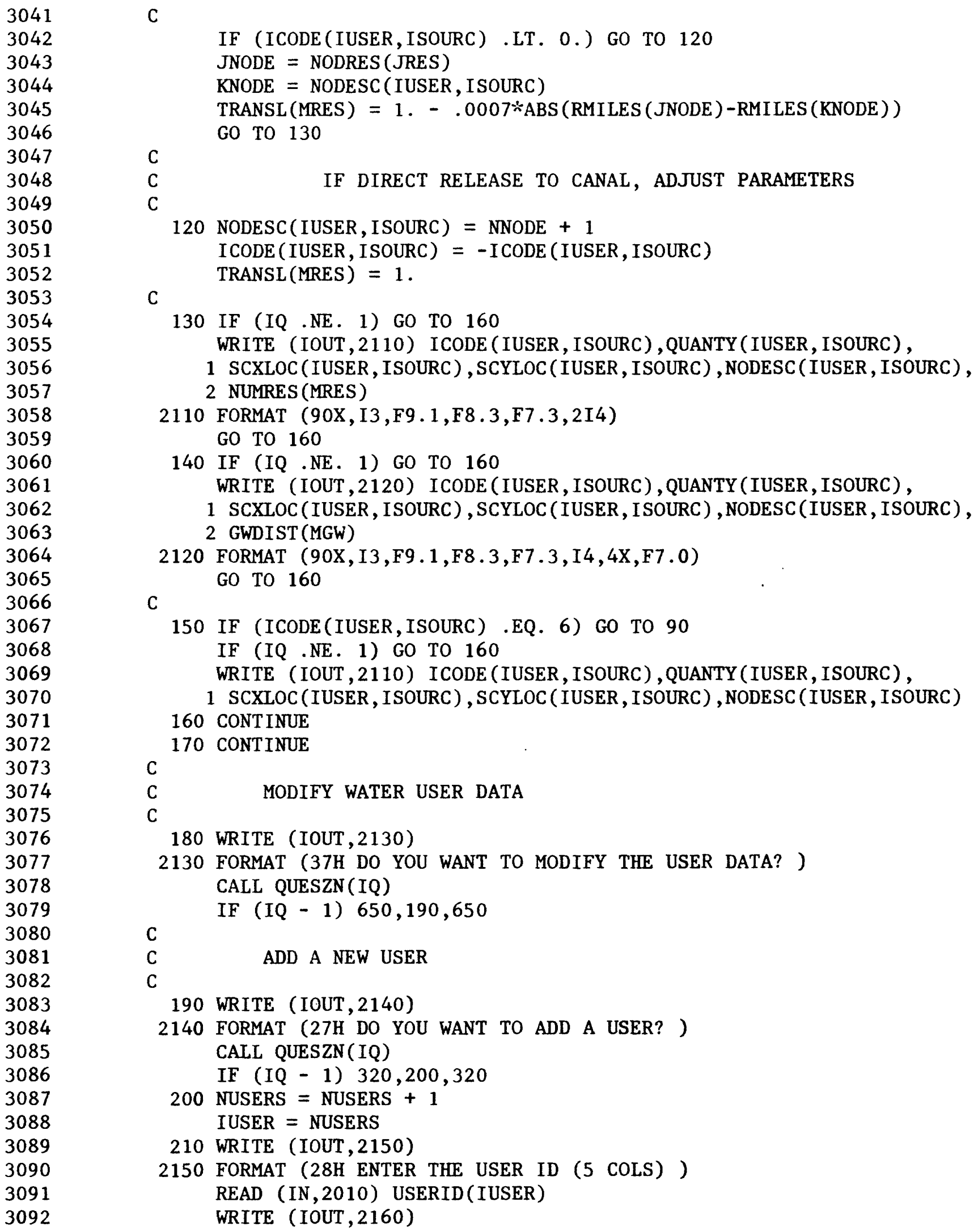

3041

3042

3043

3044

3045

3046

3047

3048

3049

3050

3051

3052

3053

3054

3055

3056

3057

3058

3059

3060

3061

3062

3063

3064

3065

3066

3067

3068

3069

3070

3071

3072

3073

3074

3075

3076

3077

3078

3079

3080

3081

3082

3083

3084

3085

3086

3087

3088

3089

3090

3091

3092

C

IF (ICODE (IUSER, ISOURC) .LT. 0.) GO TO 120

JNODE $=$ NODRES $(J R E S)$

KNODE = NODESC (IUSER, ISOURC)

$\operatorname{TRANSL}($ MRES $)=1 .-.0007 * \operatorname{ABS}(\operatorname{RMILES}($ JNODE) $)-\operatorname{RMILES}($ KNODE $))$

GO TO 130

C

$\mathrm{C}$

C

120 NODESC (IUSER, ISOURC) $=$ NNODE +1

ICODE (IUSER, ISOURC) $=-$ ICODE (IUSER, ISOURC)

TRANSL (MRES) $=1$.

C

130 IF (IQ .NE. 1) GO TO 160

WRITE (IOUT , 2110) ICODE (IUSER, ISOURC), QUANTY (IUSER, ISOURC),

1 SCXLOC (IUSER, ISOURC), SCYLOC (IUSER, ISOURC), NODESC (IUSER, ISOURC),

2 NUMRES (MRES)

2110 FORMAT ( $90 \mathrm{X}, \mathrm{I} 3, \mathrm{~F} 9.1, \mathrm{~F} 8.3, \mathrm{~F} 7.3,2 \mathrm{I} 4)$

GO TO 160

140 IF (IQ .NE. 1) GO TO 160

WRITE (IOUT , 2120) ICODE (IUSER, ISOURC), QUANTY (IUSER, ISOURC),

1 SCXIOC (IUSER, ISOURC), SCYLOC (IUSER, ISOURC), NODESC (IUSER, ISOURC), 2 GWDIST (MGW)

2120 FORMAT (90X, I3,F9.1,F8.3,F7.3, I4, 4X, F7.0)

GO TO 160

C

150 IF (ICODE (IUSER, ISOURC) .EQ. 6) GO TO 90

IF (IQ .NE. 1) GO TO 160

WRITE (IOUT , 2110) ICODE (IUSER, ISOURC), QUANTY (IUSER, ISOURC),

1 SCXLOC (IUSER, ISOURC), SCYLOC (IUSER, I SOURC), NODESC (IUSER, ISOURC)

160 CONTINUE

170 CONTINUE

C

C MODIFY WATER USER DATA

C

180 WRITE (IOUT, 2130)

2130 FORMAT (37H DO YOU WANT TO MODIFY THE USER DATA? )

CALL QUESZN(IQ)

IF (IQ - 1) $650,190,650$

$\mathrm{C}$

$\mathrm{C}$

$\mathrm{C}$

190 WRITE (IOUT, 2140)

2140 FORMAT (27H DO YOU WANT TO ADD A USER? )

CALL QUESZN (IQ)

IF (IQ - 1) $320,200,320$

200 NUSERS $=$ NUSERS +1

IUSER = NUSERS

210 WRITE (IOUT, 2150)

2150 FORMAT (28H ENTER THE USER ID ( 5 COLS) )

READ (IN, 2010) USERID (IUSER)

WRITE (IOUT, 2160) 
Attachment 8--FORTRAN Source Code of Subroutines

for Water-Supply Operations Simulation--Continued

3093

3094

3095

3096

3097

3098

3099

3100

3101

3102

3103

3104

3105

3106

3107

3108

3109

3110

3111

3112

3113

3114

3115

3116

3117

3118

3119

3120

3121

3122

3123

3124

3125

3126

3127

3128

3129

3130

3131

3132

3133

3134

3135

3136

3137

3138

3139

3140

3141

3142

3143

3144

3145

3146

3147

2160 FORMAT (30H ENTER THE USER NAME (8 COLS) )

READ (IN,2170) USRNAM (IUSER)

2170 FORMAT (A8)

WRITE (IOUT, 2180)

2180 FORMAT (3OH ENTER THE USER TYPE (5 COLS) )

READ (IN, 2010) USRTYP (IUSER)

WRITE (IOUT, 2190)

2190 FORMAT (28H ENTER THE AMOUNT (10 COLS) )

READ (IN, 2460) NUMUSR (IUSER)

WRITE (IOUT, 2200)

2200 FORMAT (35H ENTER THE DEMAND FACTOR ( 10 COLS) )

READ (IN, 2460) DMDFAC (IUSER)

WRITE (IOUT, 2210)

2210 FORMAT (34H ENTER THE X-COORDINATE (10 COLS) )

READ (IN, 2460) XLOCU (IUSER)

WRITE (IOUT, 2220)

2220 FORMAT (34H ENTER THE Y-COORDINATE (10 COLS))

READ (IN, 2460) YLOCU (IUSER)

WRITE (IOUT , 2230)

2230 FORMAT (39H ENTER THE X PLOTTING OFFSET (10 COLS) )

READ (IN, 2460) USXPLT (IUSER)

WRITE (IOUT, 2240)

2240 FORMAT (39H ENTER THE Y PLOTTING OFFSET (10 COLS) )

READ (IN, 2460) USYPLT (IUSER)

WRITE (IOUT, 2250)

2250 FORMAT (8OH ENTER THE NUMBER OF THE INDEPENDENT VARIABLE THAT HAS 1THE APPROPRIATE RAIN DATA )

READ (IN, 2010) IPRECP (IUSER)

WRITE (IOUT, 2260)

2260 FORMAT (38H ENTER THE NUMBER OF SOURCES (5 COLS) )

READ (IN, 2010) NSOURC (IUSER)

WRITE (IOUT, 2270)

2270 FORMAT (37H ENTER THE RETURN FLOW TYPE (5 COLS) )

READ (IN, 2010) RFTYPE (IUSER)

WRITE (IOUT, 2280)

2280 FORMAT (4OH ENTER THE RETURN FLOW FACTOR (10 COLS) )

READ (IN, 2460) RFVAL (IUSER)

WRITE (IOUT, 2290)

2290 FORMAT (46H ENTER THE RETURN FLOW X-COORDINATE (10 COLS) )

READ (IN, 2460) RFXLOC (IUSER)

WRITE (IOUT, 2300)

2300 FORMAT (46H ENTER THE RETURN FLOW Y-COORDINATE (10 COLS) )

READ (IN, 2460) RFYLOC (IUSER)

WRITE (IOUT, 2310)

2310 FORMAT (42H ENTER THE RETURN FLOW DISTANCE (10 COLS) )

READ (IN, 2460) RFDST

CALL CMPDST (INODE, RFXLOC (IUSER), RFYLOC (IUSER), RFDIST (IUSER))

IF (RFDST .NE. 0.) RFDIST (IUSER) = RFDST

CALL DETNOD (RFXLOC (IUSER), RFYLOC (IUSER), INODE, ISIDE)

NODERF (IUSER) = INODE

ISIDRF (IUSER) = ISIDE

IF (USRTYP (IUSER) .NE. 5) GO TO 240

DO 220 IRES $=1$, NRES

IF (RSXLOC (IRES) .EQ. XLOCU(IUSER) .AND. RSYLOC(IRES) .EQ.

1 YLOCU(IUSER)) GO TO 230 
Attachment 8--FORTRAN Source Code of Subroutines

for Water-Supply Operations Simulation--Continued

3148

3149

3150

3151

3152

3153

3154

3155

3156

3157

3158

3159

3160

3161

3162

3163

3164

3165

3166

3167

3168

3169

3170

3171

3172

3173

3174

3175

3176

3177

3178

3179

3180

3181

3182

3183

3184

3185

3186

3187

3188

3189

3190

3191

3192

3193

3194

3195

3196

3197

220 CONTINUE

WRITE (IOUT , 2070)

STOP

230 USRTYP (IUSER) $=$ RESID (IRES)

WRITE (IOUT, 2320)

2320 FORMAT (57H ENTER THE RESERVOIR SUBACCOUNT APPROPRIATE FOR THIS US 1ER )

READ (IN, 2010) IAKNT (IUSER)

240 NSORC $=$ NSOURC (IUSER)

DO 310 ISOURC $=1$, NSORC

WRITE (IOUT, 2330) ISOURC

2330 FORMAT ( $11 \mathrm{H}$ FOR SOURCE , I2,32H ENTER THE SOURCE TYPE (5 COLS) )

READ (IN, 2010) ICODE (IUSER, ISOURC)

WRITE (IOUT, 2340)

2340 FORMAT (27H ENTER THE LIMIT (10 COLS) )

READ (IN, 2460) QUANTY (IUSER, ISOURC)

WRITE (IOUT, 2350)

2350 FORMAT (41H ENTER THE SOURCE X-COORDINATE (10 COLS))

READ (IN, 2460) SCXLOC (IUSER, ISOURC)

WRITE (IOUT, 2360)

2360 FORMAT ( $41 \mathrm{H}$ ENTER THE SOURCE Y-COORDINATE (10 COLS) )

READ (IN, 2460) SCYLOC (IUSER, ISOURC)

CALL DETNOD (SCXLOC (IUSER, ISOURC), SCYLOC (IUSER, ISOURC),

1 NODESC (IUSER, ISOURC), INODE)

IF (ICODE (IUSER, ISOURC) .NE. 2) GO TO 250

C

$\mathrm{C}$

C

WRITE (IOUT, 2370)

2370 FORMAT (42H ENTER THE GROUND WATER DISTANCE (10 COLS) )

READ (IN, 2460) GWDST

$\mathrm{MGW}=\mathrm{MGW}+1$

GWUSER (MGW) = IUSER

GWSOUR (MGW) = ISOURC

INODE = NODESC (IUSER, ISOURC)

CALL CMPDST (INODE, SCXLOC (IUSER, ISOURC), SCYLOC (IUSER, ISOURC),

1 GWDIST (MGW) )

IF (GWDST .NE. 0) GWDIST (MGW) = GWDST

$\operatorname{ISDPMP}(M G W)=$ ISIDE

$\mathrm{C}$

250 IF (IABS (ICODE (IUSER, ISOURC)) .NE. 3) GO TO 300

C

C FOR RESERVOIR SOURCES

$\mathrm{C}$

WRITE (IOUT, 2380)

2380 FORMAT (33H ENTER THE RESERVOIR ID (5 COLS) )

READ (IN, 2010) NMRES

260 MRES $=$ MRES +1

RESUSR (MRES) = IUSER

RESSOR (MRES) $=$ ISOURC 
Attachment 8--FORTRAN Source Code of Subroutines

for Water-Supply Operations Simulation--Continued

3198

3199

3200

3201

3202

3203

3204

3205

3206

3207

3208

3209

3210

3211

3212

3213

3214

3215

3216

3217

3218

3219

3220

3221

$-3222$

3223

3224

3225

3226

3227

3228

3229

3230

3231

3232

3233

3234

3235

3236

3237

3238

3239

3240

3241

3242

3243

3244

3245

3246
C

DO 270 JRES $=1$, NRES

IF (NMRES .EQ. RESID(JRES)) G

270 CONTINUE

STOP

C

280 NUMRES (MRES) $=$ JRES

WRITE (IOUT, 2390)

2390 FORMAT (41H ENTER THE RESERVOIR SUB-ACCOUNT (5 COLS) )

READ (IN, 2010) IACCNT

IF (IACCNT .EQ. 0) IACCNT $=1$

ISBACT (MRES) = IACCNT

C

C

C

C

$\mathrm{C}$

C

290 NODESC (IUSER, ISOURC) $=$ NNODE +1

ICODE (IUSER, ISOURC) $=-$ ICODE (IUSER, ISOURC)

TRANSL (MRES) $=1$.

C

GO TO 310

C

300 IF (ICODE (ISIDE, IUSER) .EQ. 6) GO TO 260

310 CONTINUE

GO TO 180

C

C CHANGE WATER USER DATA

C

320 WRITE (IOUT, 2400)

2400 FORMAT (47H DO YOU WANT TO CHANGE ALL THE DATA FOR A USER? ) CALL QUESZN (IQ)

IF (IQ - 1) $530,330,530$

330 WRITE (IOUT, 2410)

2410 FORMAT (18H ENTER THE USER ID )

READ (IN, 2010) IUSID

DO 340 IUSER $=1$, NUSERS

IF (IUSID .EQ. USERID(IUSER)) GO TO 360

340 CONTINUE

350 WRITE (IOUT, 2420) IUSID

2420 FORMAT (6H USER , I5, 14H WAS NOT FOUND )

GO TO 320

360 WRITE (IOUT, 2430) USRNAM (IUSER) 
Attachment 8--FORTRAN Source Code of Subroutines

for Water-Supply Operations Simulation--Continued

3247

3248

3249

3250

3251

3252

3253

3254

3255

3256

3257

3258

3259

3260

3261

3262

3263

3264

3265

3266

3267

3268

3269

3270

3271

3272

3273

3274

3275

3276

3277

3278

3279

3280

3281

3282

3283

3284

3285

3286

3287

3288

3289

3290

3291

3292

3293

3294

3295

3296

3297

3298

3299

2430 FORMAT (74H IF THE VALUE DISPLAYED AFTER THE REQUEST IS ACCEPTABLE

1, MERELY TYPE <CR> ,//, 30H ENTER THE USER NAME ( 8 COLS) ,/, A8)

READ (IN, 2170) NAME 1

IF (NAME 1 .NE. '') USRNAM(IUSER) = NAME 1

WRITE (IOUT, 2180)

WRITE (IOUT, 2010) USRTYP(IUSER)

READ (IN, 2010) ITEMP

IF (ITEMP .NE. 0) USRTYP(IUSER) = ITEMP

WRITE (IOUT, 2190)

WRITE (IOUT, 2460) NUMUSR (IUSER)

READ (IN, 2460) TEMP

IF (TEMP .NE. 0.) NUMUSR (IUSER) = TEMP

WRITE (IOUT, 2200)

WRITE (IOUT, 2460) DMDFAC (IUSER)

READ (IN, 2460) TEMP

IF (TEMP .NE. 0.) DMDFAC (IUSER) = TEMP

WRITE (IOUT, 2210)

WRITE (IOUT, 2460) XLOCU (IUSER)

READ (IN, 2460) TEMP

IF (TEMP .NE. 0.) XLOCU(IUSER) = TEMP

WRITE (IOUT, 2220)

WRITE (IOUT, 2460) YLOCU(IUSER)

READ (IN, 2460) TEMP

IF (TEMP .NE. 0.) YLOCU (IUSER) = TEMP

WRITE (IOUT, 2230)

WRITE (IOUT, 2460) USXPLT (IUSER)

READ (IN, 2460) TEMP

IF (TEMP .NE. 0.) USXPLT (IUSER) = TEMP

WRITE (IOUT, 2240)

WRITE (IOUT, 2460) USYPLT (IUSER)

READ (IN, 2460) TEMP

IF (TEMP .NE. 0.) USYPLT (IUSER) = TEMP

WRITE (IOUT , 2250)

WRITE (IOUT, 2010) IPRECP (IUSER)

READ (IN , 2010) ITEMP

IF (ITEMP .NE. 0) IPRECP (IUSER) = ITEMP

WRITE (IOUT, 2260)

WRITE (IOUT, 2010) NSOURC (IUSER)

READ (IN, 2010) ITEMP

IF (ITEMP . NE. 0) NSOURC (IUSER) = ITEMP

WRITE (IOUT, 2270)

WRITE (IOUT, 2010) RFTYPE (IUSER)

READ (IN, 2010) ITEMP

IF (ITEMP .NE. 0) RFTYPE (IUSER) = ITEMP

WRITE (IOUT, 2280)

WRITE (IOUT, 2460) RFVAL (IUSER)

READ (IN, 2460) TEMP

IF (TEMP .NE. 0.) RFVAL (IUSER) = TEMP

WRITE (IOUT, 2290)

WRITE (IOUT, 2460) RFXLOC (IUSER)

READ (IN, 2460) TEMP

IF (TEMP .NE. 0.) RFXLOC (IUSER) = TEMP

WRITE (IOUT, 2300) 
Attachment 8--FORTRAN Source Code of Subroutines

for Water-Supply Operations Simulation--Continued

3300

3301

3302

3303

3304

3305

3306

3307

3308

3309

3310

3311

3312

3313

3314

3315

3316

3317

3318

3319

3320

3321

3322

3323

3324

3325

3326

3327

3328

3329

3330

3331

3332

3333

3334

3335

3336

3337

3338

3339

3340

3341

3342

3343

3344

3345

3346

3347

3348

3349

WRITE (IOUT, 2460) RFYLOC (IUSER)

READ (IN, 2460) TEMP

IF (TEMP .NE. 0.) RFYLOC (IUSER) = TEMP

WRITE (IOUT, 2310)

WRITE (IOUT, 2460) RFDIST (IUSER)

READ (IN, 2460) TEMP

IF (TEMP .NE. O.) RFDIST (IUSER) = TEMP

CALL DETNOD (RFXLOC (IUSER), RFYLOC (IUSER), INODE, ISIDE)

NODERF (IUSER) = INODE

ISIDRF (IUSER) = ISIDE

IF (USRTYP(IUSER) .NE. 5) GO TO 390

DO 370 IRES $=1$, NRES

IF (RSXLOC (IRES) .EQ. XLOCU (IUSER) .AND. RSYLOC (IRES) .EQ.

1 YLOCU(IUSER)) GO TO 380

370 CONTINUE

WRITE (IOUT, 2070)

STOP

380 USRTYP (IUSER) = RESID (IRES)

WRITE (IOUT , 2320)

WRITE (IOUT, 2010) IAKNT (IUSER)

READ (IN, 2010) ITEMP

IF (ITEMP .NE. O) IAKNT (IUSER) = ITEMP

390 NSORC $=$ NSOURC (IUSER)

DO 520 ISOURC $=1$, NSORC

WRITE (IOUT, 2330) ISOURC

WRITE (IOUT, 2010) ICODE (IUSER, ISOURC)

READ (IN, 2010) ITEMP

IF (ITEMP .NE . 0) ICODE (IUSER, ISOURC) = ITEMP

WRITE (IOUT , 2340)

WRITE (IOUT, 2460) QUANTY (IUSER, ISOURC)

READ (IN, 2460) TEMP

IF (TEMP .NE . 0.) QUANTY (IUSER, ISOURC) = TEMP

WRITE (IOUT, 2350)

WRITE (IOUT, 2460) SCXLOC (IUSER, ISOURC)

READ (IN, 2460) TEMP

IF (TEMP .NE. 0.) SCXLOC (IUSER, ISOURC) = TEMP

WRITE (IOUT, 2360)

WRITE (IOUT, 2460) SCYLOC (IUSER, ISOURC)

READ (IN, 2460) TEMP

IF (TEMP .NE . 0.) SCYLOC (IUSER, ISOURC) = TEMP

CALL DETNOD (SCXLOC (IUSER, ISOURC), SCYLOC (IUSER, ISOURC),

1 NODESC (IUSER, ISOURC), INODE)

IF (ICODE (IUSER, ISOURC) .NE. 2) GO TO 420

$\mathrm{C}$

$\mathrm{C}$

$\mathrm{C}$

FOR GROUND-WATER SOURCES

DO $400 \mathrm{JGW}=1, \mathrm{MGW}$

IF (GWUSER(JGW) .EQ. IUSER .AND. GWSOUR(JGW) .EQ. ISOURC)

1 GO TO 410

400 CONTINUE 
Attachment 8--FORTRAN Source Code of Subroutines

for Water-Supply Operations Simulation--Continued

410 WRITE (IOUT, 2370)

WRITE (IOUT, 2010) GWDIST (JGW)

READ (IN, 2460) TEMP

IF (TEMP .NE. 0) GWDST = TEMP

CALL CMPDST (INODE, SCXLOC (IUSER, ISOURC), SCYLOC (IUSER, ISOURC),

1 GWDIST(JGW) )

IF (GWDST .NE. 0) GWDIST (JGW) = GWDST

420 IF (IABS (ICODE (IUSER, ISOURC)) .NE. 3) GO TO 510

C

C FOR RESERVOIR SOURCES

C

430 DO 440 JRES $=1$, MRES

IF (RESUSR (JRES) .EQ. IUSER .AND. RESSOR(JRES) .EQ. ISOURC)

1 GO TO 450

440 CONT INUE

450 WRITE (IOUT, 2380)

WRITE (IOUT, 2010) NUMRES (JRES)

READ (IN , 2010) ITEMP

IF (ITEMP .EQ. 0) GO TO 480

NMRES = ITEMP

DO 460 IRES $=1$, NRES

IF (NMRES .EQ. RESID(IRES)) GO TO 470

460 CONTINUE

STOP

C

470 NUMRES (JRES) = IRES

480 WRITE (IOUT, 2390)

WRITE (IOUT, 2010) ISBACT (JRES)

READ (IN, 2010) ITEMP

IF (ITEMP .EQ. 0) GO TO 490

ISBACT $(J R E S)=$ ITEMP

$\mathrm{C}$

C

C

490 IF (ICODE (IUSER, ISOURC) .LT. 0) GO TO 500

JNODE $=$ NODRES (IRES)

KNODE $=$ NODESC (IUSER, ISOURC)

$\operatorname{TRANSL}($ JRES $)=1 .-.0007 *$ ABS $(\operatorname{RMILES}($ JNODE) $-\operatorname{RMILES}($ KNODE $))$ GO TO 520

C

$\mathrm{C}$

C

500 NODESC (IUSER, ISOURC) $=$ NNODE +1

ICODE (IUSER, ISOURC) $=$-ICODE (IUSER, ISOURC)

TRANSL (JRES) $=1$.

C

GO TO 520

510 IF (ICODE (IUSER, ISOURC) .EQ. 6) GO TO 430

520 CONTINUE

GO TO 180

C

C CHANGE A DEMAND FACTOR 
Attachment 8--FORTRAN Source Code of Subroutines

for Water-Supply Operations Simulation--Continued

3402

3403

3404

3405

3406

3407

3408

3409

3410

3411

3412

3413

3414

3415

3416

3417

3418

3419

3420

3421

3422

3423

3424

3425

3426

3427

3428

3429

3430

3431

3432

3433

3434

3435

3436

3437

3438

3439

3440

3441

3442

3443

3444

3445

3446

3447

3448

3449

3450

3451
C

530 WRITE (IOUT, 2440)

2440 FORMAT (51H DO YOU WANT TO CHANGE THE DEMAND FACTOR OF A USER?) CALL QUESZN(IQ) IF (IQ - 1) $570,540,570$

540 WRITE (IOUT, 2410)

READ (IN, 2010) IUSID

DO 550 IUSER $=1$, NUSERS

IF (IUSID .EQ. USERID(IUSER)) GO TO 560

550 CONTINUE

WRITE (IOUT, 2420) IUSID

GO TO 540

560 WRITE (IOUT, 2450)

2450 FORMAT (42H ENTER THE NEW DEMAND FACTOR (10 COLUMNS) )

READ (IN , 2460) DMDFAC (IUSER)

2460 FORMAT (F15.3)

GO TO 180

570 WRITE (IOUT, 2470)

2470 FORMAT (56H DO YOU WANT TO CHANGE THE RETURN FLOW FACTOR OF A USER $1 ?$ )

CALI QUESZN(IQ)

IF (IQ - 1) $610,580,610$

580 WRITE (IOUT, 2410)

READ (IN, 2010) IUSID

DO 590 IUSER = 1 , NUSERS

IF (IUSID .EQ. USERID(IUSER)) GO TO 600

590 CONTINUE

WRITE (IOUT, 2420) IUSID

GO TO 580

600 WRITE (IOUT, 2480)

2480 FORMAT (47H ENTER THE NEW RETURN FLOW FACTOR (10 COLUMNS) )

READ (IN, 2460) RFVAL (IUSER)

GO TO 180

610 WRITE (IOUT, 2490)

2490 FORMAT (45H DO YOU WANT TO CHANGE THE SOURCES OF A USER? )

CALI QUESZN(IQ)

IF (IQ - 1) $650,620,650$

620 WRITE (IOUT, 2500)

2500 FORMAT (63H ENTER THE USER ID (5 COLUMNS) AND HOW MANY SOURCES (5 1COLUMNS) )

READ (IN , 2510) IUSID, NSORC

2510 FORMAT (16I5)

DO 630 IUSER $=1$, NUSERS

IF (IUSID .EQ. USERID(IUSER)) GO TO 640

630 CONTINUE

GO TO 350

640 NSOURC (IUSER) $=$ NSORC

GO TO 390

$\mathrm{C}$

C WRITE WATER USER DATA 
Attachment 8--FORTRAN Source Code of Subroutines for Water-Supply Operations Simulation--Continued

3452

3453

3454

3455

3456

3457

3458

3459

3460

3461

3462

3463

3464

3465

3466

3467

3468

3469

3470

3471

3472

3473

3474

3475

3476

3477

3478

3479

3480

3481

3482

3483

3484

3485

3486

3487

3488

3489

3490

3491

3492

3493

3494

3495

3496

3497

3498

3499

3500

3501

3502

3503

3504

3505

3506
C

650 WRITE $(52,2040)$

DO 740 IUSER $=1$, NUSERS

IF (USRTYP(IUSER) .LE. 5) GO TO 660

ITEMP $=5$

WRITE $(52,2080)$ IUSER, USERID (IUSER), USRNAM(IUSER), ITEMP,

1 NUMUSR (IUSER), DMDFAC (IUSER), XLOCU (IUSER), YLOCU (IUSER),

2 IAKNT (IUSER), RFTYPE (IUSER), RFVAL (IUSER), RFXLOC (IUSER),

3 RFYLOC (IUSER), NODERF (IUSER), RFDIST (IUSER), NSOURC (IUSER),

4 USRTYP (IUSER)

GO TO 670

660 WRITE $(52,2060)$ IUSER, USERID(IUSER), USRNAM(IUSER), USRTYP(IUSER),

1 NUMUSR (IUSER), DMDFAC (IUSER), XLOCU (IUSER), YLOCU (IUSER),

2 IPRECP (IUSER), RFTYPE (IUSER), RFVAL (IUSER), RFXLOC (IUSER),

3 RFYLOC (IUSER), NODERF (IUSER), RFDIST (IUSER), NSOURC (IUSER)

670 NSORC $=$ NSOURC $($ IUSER $)$

DO 740 ISOURC $=1$, NSORC

IF (IABS (ICODE (IUSER, ISOURC)) .EQ. 3) GO TO 710

IF (ICODE (IUSER, ISOURC) .EQ. 6) GO TO 710

IF (ICODE (IUSER, ISOURC) .EQ. 2) GO TO 680

WRITE $(52,2110)$ ICODE (IUSER, ISOURC), QUANTY (IUSER, ISOURC),

1 SCXLOC (IUSER, ISOURC), SCYLOC (IUSER, ISOURC), NODESC (IUSER, ISOURC)

GO TO 740

680 DO $690 \mathrm{JGW}=1, \mathrm{MGW}$

IF (GWUSER(JGW) .EQ. IUSER .AND. GWSOUR(JGW) .EQ. ISOURC)

1 GO TO 700

690 CONTINUE

700 WRITE $(52,2120)$ ICODE (IUSER, ISOURC), QUANTY (IUSER, ISOURC),

1 SCXLOC (IUSER, ISOURC), SCYLOC (IUSER, ISOURC), NODESC (IUSER, ISOURC),

2 GWDIST(JGW)

GO TO 740

710 DO 720 JRES $=1$, MRES

IF (RESUSR(JRES) .EQ. IUSER .AND. RESSOR(JRES) .EQ. ISOURC)

1 GO TO 730

720 CONTINUE

730 WRITE $(52,2110)$ ICODE (IUSER, ISOURC), QUANTY (IUSER, ISOURC),

1 SCXLOC (IUSER, ISOURC), SCYLOC (IUSER, ISOURC), NODESC (IUSER, ISOURC),

2 NUMRES (JRES)

740 CONTINUE

$\mathrm{C}$

C PLOT WATER USER LOCATIONS

$\mathrm{C}$

WRITE (IOUT, 2520)

2520 FORMAT(55H DO YOU WANT TO PLOT THE WATER USER LOCATION SCHEMATIC?) CALL QUESZN(IQ)

IF (IQ-1) $760,750,760$

750 WRITE (IDUM, 2530) TITL

2530 FORMAT (5A4,24H - WATER USER LOCATIONS\$)

CALL PLTUSR(ITITLE)

$\mathrm{C}$

C

C

760 READ $(51,2000)$ TITLER

READ (51,2010) NRIGHT

WRITE $(52,2540)$ TITLER, NRIGHT 
Attachment 8--FORTRAN Source Code of Subroutines

for Water-Supply Operations Simulation--Continued

3507

3508

3509

3510

3511

3512

3513

3514

3515

3516

3517

3518

3519

3520

3521

3522

3523

3524

3525

3526

3527

3528

3529

3530

3531

3532

3533

3534

3535

3536

3537

3538

3539

3540

3541

3542

3543

3544

3545

3546

3547

3548

3549

3550

3551

3552

3553

3554

3555

3556

3557

3558

3559

3560

3561

3562

2540 FORMAT (19H1WATER RIGHT FILE: ,20A4,9X,11H, CONTAINS , I3,7H RIGHTS $1, / / /, 50 \mathrm{H}$ OWNER OWNER AMTE 2 ID NAME )

DO 770 IUSER $=1$, NUSERS

DO 770 ISOURC $=1,15$

770 USED (IUSER, ISOURC) $=0$

WRITE (IOUT, 2550)

2550 FORMAT (41H DO YOU WANT TO SEE THE WATER RIGHT FILE? ) CALL QUESZN(IQ)

IF (IQ .EQ. 1) WRITE (IOUT, 2560)

2560 FORMAT $(/ / /, 31 \mathrm{H}$ RIGHT OWNER OWNER AMOUNT , /, 9X, 1 12HID NAME )

DO 780 IRIGHT $=1$, NRIGHT

READ (51,2570) OWNER (IRIGHT), NAME (IRIGHT), DATE (IRIGHT) , AMNT (IRIGHT)

2570 FORMAT (I5, A8, 2X, A8,F10.0)

IF (IQ .EQ. 0) GO TO 780

WRITE (IOUT , 2580) IRIGHT, OWNER (IRIGHT), NAME (IRIGHT), AMNT (IRIGHT)

2580 FORMAT $(\mathrm{I} 5, \mathrm{I} 8,2 \mathrm{X}, \mathrm{A} 8, \mathrm{~F} 10.1)$

780 CONTINUE

$\mathrm{C}$

DO 850 IRIGHT $=1$, NRIGHT

790 DO 800 IUSER $=1$, NUSERS

IF (OWNER (IRIGHT) .EQ. USERID (IUSER)) GO TO 810

800 CONTINUE

WRITE (IOUT, 2590) IRIGHT, OWNER (IRIGHT)

2590 FORMAT (5X,11H FOR RIGHT ,I3,11H, OWNER ID ,A8,

$127 \mathrm{H}$ DOES NOT MATCH ANY USER ID ,/, 76H DO YOU WANT TO CHANGE IT?

2 (HAVING AN EXTRA RIGHT HAS NO DELETERIOUS EFFECT) )

CALL QUESZN(IQ)

IF (IQ .EQ. 0) GO TO 850

WRITE (IOUT, 2600)

2600 FORMAT (32H ENTER NEW OWNER ID, (5 COLUMNS) )

READ (IN, 2010) OWNER (IRIGHT)

GO TO 790

810 ISOURC $=0$

820 ISOURC $=$ ISOURC +1

IF (ISOURC .LE. NSOURC(IUSER)) GO TO 830

WRITE (IOUT, 2610) USERID(IUSER), IRIGHT

2610 FORMAT (6H USER , I5,34H DOES NOT HAVE A SOURCE FOR RIGHT ,I3 ,/,

$179 \mathrm{H}$ DO YOU WANT TO ADD A SOURCE? (HAVING AN EXTRA RIGHT HAS NO DE

2LETERIOUS EFFECT) )

CALL QUESZN(IQ)

IF (IQ .EQ. 0) GO TO 850

NSOURC (IUSER) = ISOURC

WRITE (IOUT, 2330) ISOURC

READ (IN, 2010) ICODE (IUSER, ISOURC)

WRITE (IOUT, 2340)

READ (IN, 2460) QUANTY (IUSER, ISOURC)

WRITE (IOUT, 2350)

READ (IN, 2460) SCXLOC (IUSER, I SOURC)

WRITE (IOUT, 2360)

READ (IN, 2460) SCYLOC (IUSER, ISOURC)

CALL DETNOD (SCXIOC (IUSER, ISOURC), SCYLOC (IUSER, ISOURC),

1 NODESC (IUSER, ISOURC), ISIDE)

USED (IUSER, ISOURC) $=1$

GO TO 850 
Attachment 8--FORTRAN Source Code of Subroutines

for Water-Supply Operations Simulation--Continued

3563

3564

3565

3566

3567

3568

3569

3570

3571

3572

3573

3574

3575

3576

3577

3578

3579

3580

3581

3582

3583

3584

3585

3586

3587

3588

3589

3590

3591

3592

3593

3594

3595

3596

3597

3598

3599

3600

3601

3602

3603

3604

3605

3606

3607

3608

3609

3610

3611

3612

3613

3614

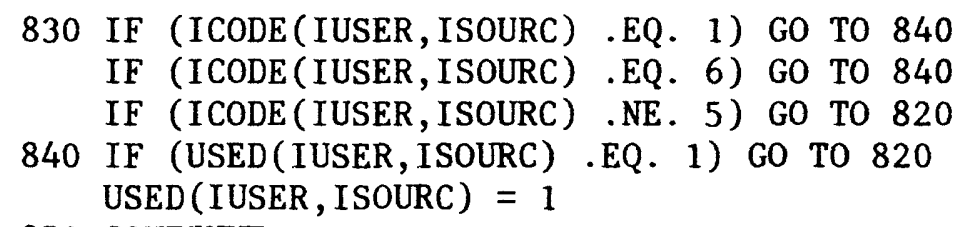

830 IF (ICODE (IUSER, ISOURC) .EQ. 1) GO TO 840

IF (ICODE (IUSER, ISOURC) .EQ. 6) GO TO 840

IF (ICODE (IUSER, ISOURC) .NE. 5) GO TO 820

840 IF (USED (IUSER, ISOURC) .EQ. 1) GO TO 820

USED (IUSER, ISOURC) $=1$

850 CONTINUE

C

DO 880 IUSER $=1$, NUSERS

NSOUR $=$ NSOURC (IUSER)

DO 880 ISOURC $=1$, NSOUR

IF (ICODE (IUSER, ISOURC) .EQ. 1) GO TO 860

IF (ICODE (IUSER, ISOURC) .EQ. 6) GO TO 860

IF (ICODE (IUSER, ISOURC) .NE. 5) GO TO 880

860 IF (USED (IUSER, ISOURC) .EQ. 1 .OR. QUANTY(IUSER, ISOURC) .EQ. 0.)

1 GO TO 880

WRITE (IOUT , 2620) IUSER, USERID (IUSER), ISOURC, QUANTY (IUSER, ISOURC)

2620 FORMAT (6H USER , I3,7H (ID $=, \mathrm{I} 5,10 \mathrm{H})$, SOURCE , I3,

1 19H, (WITH A LIMIT OF ,F10.1,13H HAS NO RIGHT ,/,

2 79H DO YOU WANT TO ADD A RIGHT? (A SOURCE WITH NO RIGHT HAS NO DE

3LETERIOUS EFFECT) )

CALL QUESZN(IQ)

IF (IQ .EQ. 0) GO TO 880

WRITE (IOUT, 2630)

2630 FORMAT (44H ENTER THE NUMERICAL NUMBER OF THE NEW RIGHT )

READ (IN, 2010) IRIGHT

MRIGHT = NRIGHT - IRIGHT - 1

DO $870 \mathrm{KRIGHT}=1$, MRIGHT

JRIGHT = NRIGHT - KRIGHT +1

870 OWNER (JRIGHT +1 ) = OWNER (JRIGHT)

OWNER (IRIGHT) = USERID (IUSER)

NRIGHT $=$ NRIGHT +1

880 CONTINUE

$\mathrm{C}$

C WRITE WATER RIGHTS

$\mathrm{C}$

DO 890 IRIGHT $=1$, NRIGHT

890 WRITE $(52,2640)$ OWNER (IRIGHT), NAME (IRIGHT), DATE (IRIGHT),

1 AMNT (IRIGHT)

2640 FORMAT ( $5 \mathrm{X}, \mathrm{I} 5,5 \mathrm{X}, \mathrm{A} 8,5 \mathrm{X}, \mathrm{A} 8,2 \mathrm{X}, \mathrm{F} 12.2)$

C

C ENTER FREQUENCY OF PLOTTING WATER USER SCHEMATIC

$\mathrm{C}$

WRITE (IOUT , 2650)

2650 FORMAT (65H ENTER THE FREQUENCY OF PLOTTING THE WATER USE SCHEMATI 1C (5 COLS) ,/,7X, 18HO FOR NO SCHEMATIC,/,7X,15H12 FOR ANNUALLY,/, 2 7X,34H99999 FOR PERIOD-OF-RECORD AVERAGE ,/, 45H AND ENTER THE MA 3XIMUM ANNUAL USAGE (10 COLS) )

C

READ (IN, 2090) ISKMU, UMAX

C ENTER WATER USERS FOR HYDROGRAPHS

WRITE（IOUT , 2660) 
Attachment 8--FORTRAN Source Code of Subroutines

for Water-Supply Operations Simulation--Continued

3615

3616

3617

3618

3619

3620

3621

3622

3623

3624

3625

3626

3627

3628

3629

3630

3631

3632

3633

3634

3635

3636

3637

3638

3639

3640

3641

3642

3643

3644

3645

3646

3647

3648

3649

3650

3651

3652

3653

3654

3655

3656
2660 FORMAT (71H ENTER THE WATER USER IDS FOR THOSE USERS AT WHICH YOU 1WANT HYDROGRAPHS $, /, 10 \mathrm{X}, 44 \mathrm{H} \cdots$ NOTE* 99999 WILL PROVIDE BASINWIDE T 2OTAL ,/,7X,32H(5 COLS EACH - LIMIT OF 5 NODES) )

READ (IN , 2510) IHYU

DO $930 I=1,5$

IF (IHYU(I) .EQ. 99999) GO TO 910

DO $900 \mathrm{~J}=1$, NUSERS

IF (IHYU(I) .NE. USERID(J)) GO TO 900

$\operatorname{IHYU}(\mathrm{I})=\mathrm{J}$

GO TO 930

900 CONTINUE

GO TO 930

910 DO $920 \mathrm{~J}=1,600$

$\operatorname{UHYD}(\mathrm{J}, \mathrm{I})=0$.

$\operatorname{UHYP}(\mathrm{J}, \mathrm{I})=0$.

$920 \operatorname{UHYT}(J, I)=0$.

930 CONTINUE

$\mathrm{C}$

C ENTER DESIRED WATER USERS FOR MONTHLY TABLES

C

WRITE (IOUT, 2670)

2670 FORMAT (73H ENTER THE WATER USER IDS FOR THOSE USERS AT WHICH YOU 1WANT MONTHLY USAGE, /, 10X,44H*NOTE*\% 99999 WILL PROVIDE BASINWIDE

2 TOTAL , /,7X,32H( 5 COLS EACH - LIMIT OF 5 NODES) )

READ (IN, 2510) ITBLU

DO $970 I=1,5$

IF (ITBLU(I) .EQ. 99999) GO TO 950

DO $940 \mathrm{~J}=1$, NUSERS

IF (ITBLU(I) .NE. USERID(J)) GO TO 940

$\operatorname{ITBLU}(\mathrm{I})=\mathrm{J}$

GO TO 970

940 CONTINUE

GO TO 970

950 DO $960 \mathrm{~J}=1,600$

$\operatorname{IUTBLD}(I, J)=0$

$\operatorname{IUTBLP}(I, J)=0$

$960 \operatorname{IUTBLT}(\mathrm{I}, \mathrm{J})=0$

970 CONTINUE

$\mathrm{C}$

C

C

ENTER WATER USERS FOR STATISTICAL SUMMARIES

WRITE (IOUT, 2680) 
Attachment 8--FORTRAN Source Code of Subroutines

\section{for Water-Supply Operations Simulation--Continued}

3657

3658

3659

3660

3661

3662

3663

3664

3665

3666

3667

3668

3669

3670

3671

3672

3673

3674

3675

3676

3677

3678

3679

3680

3681

3682

3683

3684

3685

3686

3687

3688

3689

3690

3691

3692

3693

3694

3695

3696

3697

3698

3699

3700

3701

3702

3703

3704

3705

3706

3707

2680 FORMAT (70H ENTER THE WATER USER IDS FOR THOSE USERS AT WHICH YOU

IWANT STATISTICS $, /, 10 \mathrm{X}, 44 \mathrm{H} * *$ NOTE** 99999 WILL PROVIDE BASINWIDE TO 2TAL , /,7X,33H(5 COLS EACH - LIMIT OF 10 NODES) )

READ (IN, 2510) ISSU

DO $1000 \mathrm{I}=1,10$

IF (ISSU(I) .LE. 0) GO TO 1000

DO $980 \mathrm{~J}=1,12$

$\operatorname{UMENND}(I, J)=0$.

$\operatorname{UMENDD}(\mathrm{I}, \mathrm{J})=0$.

$\operatorname{UMENGW}(I, J)=0$.

$\operatorname{UMENRR}(I, J)=0$.

$\operatorname{UMENTA}(I, J)=0$.

$\operatorname{UMENP}(I, J)=0$.

$\operatorname{UMENWC}(I, J)=0$.

$\operatorname{UMENC}(\mathrm{I}, \mathrm{J})=0$.

$\operatorname{UMENCL}(I, J)=0$.

$\operatorname{UMENTW}(I, J)=0$.

$980 \operatorname{UMENDP}(\mathrm{I}, \mathrm{J})=0$.

DO $990 \mathrm{~J}=1$, NUSERS

IF (ISSU(I) .NE. USERID(J)) GO TO 990

$\operatorname{ISSU}(\mathrm{I})=\mathrm{J}$

GO TO 1000

990 CONTINUE

1000 CONTINUE

C

C ENTER RESERVOIRS FOR HYDROGRAPHS

C

WRITE (IOUT , 2690)

2690 FORMAT (75H ENTER THE RESERVOIR IDS FOR THOSE RESERVOIRS AT WHICH IYOU WANT HYDROGRAPHS , /,7X,32H(5 COLS EACH - LIMIT OF 5 NODES) )

READ (IN, 2510) IHYR

DO $1020 \mathrm{I}=1,5$

DO $1010 \mathrm{~J}=1$, NRES

IF (IHYR(I) .NE. RESID(J)) GO TO 1010

$\operatorname{IHYR}(\mathrm{I})=\mathrm{J}$

GO TO 1020

1010 CONTINUE

1020 CONTINUE

C

C

$\mathrm{C}$

\section{ENTER RESERVOIRS FOR MONTHLY TABLES}

WRITE (IOUT, 2700)

2700 FORMAT (8OH ENTER THE RESERVOIR IDS FOR THOSE RESERVOIRS AT WHICH IYOU WANT MONTHLY CONTENTS, $/, 7 \mathrm{X}, 32 \mathrm{H}$ ( 5 COLS EACH - LIMIT OF 5 NODES)

2 )

READ (IN, 2510) ITBLR

DO $1040 \mathrm{I}=1,5$

DO $1030 \mathrm{~J}=1$, NRES

IF (ITBLR(I) .NE. RESID(J)) GO TO 1030

$\operatorname{ITBLR}(\mathrm{I})=\mathrm{J}$

GO TO 1040 
Attachment 8--FORTRAN Source Code of Subroutines

for Water-Supply Operations Simulation--Continued

1030 CONTINUE

C

C ENTER RESERVOIRS FOR STATISTICAL SUMMARIES

C WRITE (IOUT, 2710)

2710 FORMAT (74H ENTER THE RESERVOIR IDS FOR THOSE RESERVOIRS AT WHICH IYOU WANT STATISTICS, /,7X,33H( 5 COLS EACH - LIMIT OF 10 NODES) ) READ (IN, 2510) ISSR

DO $1070 I=1,10$

IF (ISSR(I) .LE. 0) GO TO 1070

DO $1050 \mathrm{~J}=1,12$

$\operatorname{RMEANS}(I, J)=0$.

$\operatorname{RMEANC}(I, J)=0$.

$\operatorname{RMEANR}(I, J)=0$.

$\operatorname{RMEAND}(I, J)=0$.

$\operatorname{RMEANI}(I, J)=0$.

$1050 \operatorname{RMEANE}(I, J)=0$.

DO $1060 \mathrm{~J}=1$, NRES

IF (ISSR(I) .NE. RESID(J)) GO TO 1060 $\operatorname{ISSR}(\mathrm{I})=\mathrm{J}$ GO TO 1070

1060 CONTINUE

1070 CONTINUE

C

RETURN

END 
Attachment 8--FORTRAN Source Code of Subroutines

for Water-Supply Operations Simulation--Continued

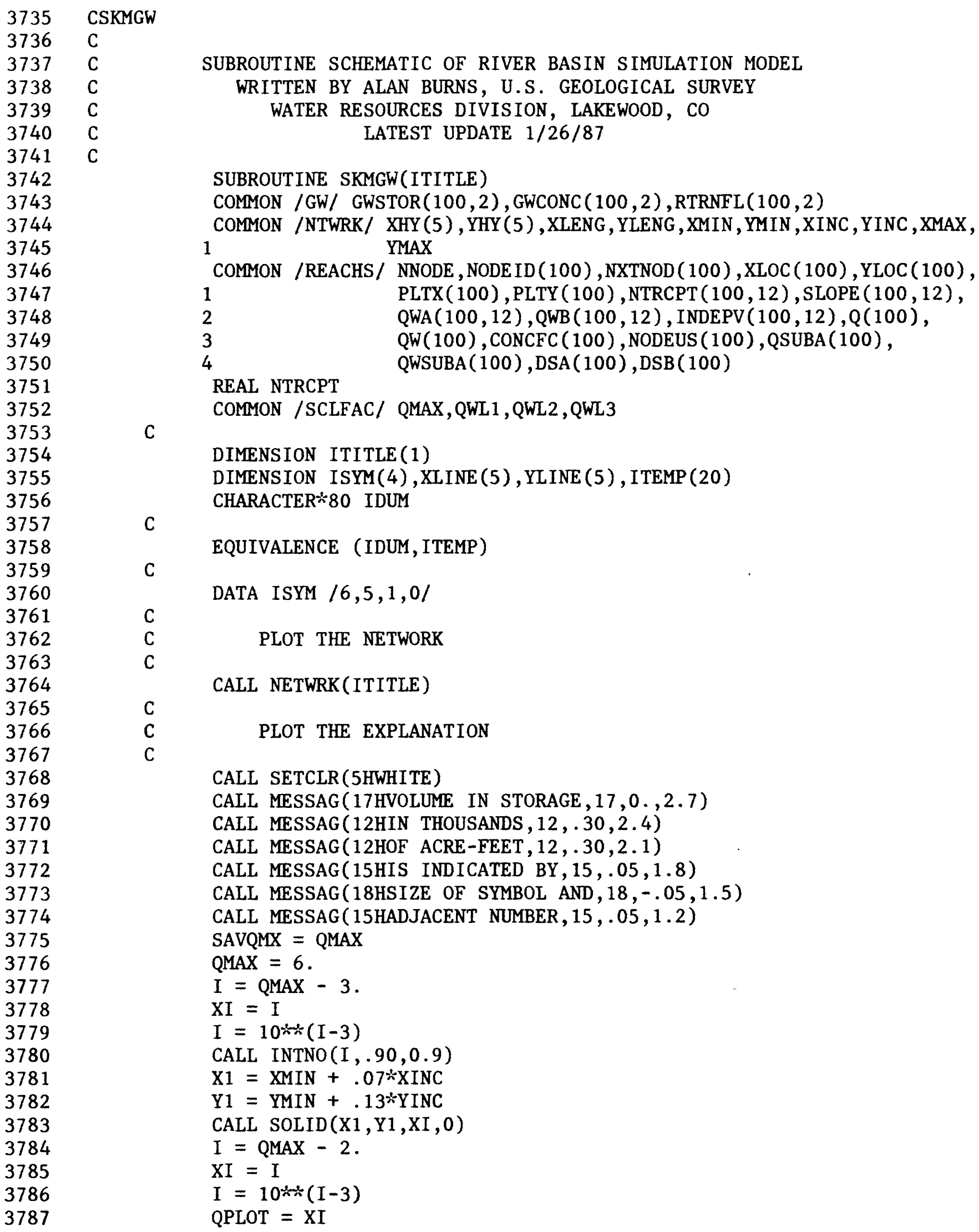


Attachment 8--FORTRAN Source Code of Subroutines

for Water-Supply Operations Simulation--Continued

3788

3789

3790

3791

3792

3793

3794

3795

3796

3797

3798

3799

3800

3801

3802

3803

3804

3805

3806

3807

3808

3809

3810

3811

3812

3813

3814

3815

3816

3817

3818

3819

3820

3821

3822

3823

3824

3825

3826

3827

3828

3829

3830

3831

3832

3833

3834

3835

CALL INTNO $(I, .93,0.55)$

Y1 $=$ YMIN +.07*YINC

CALL SOLID $(\mathrm{X} 1, \mathrm{Y} 1, \mathrm{XI}, 0)$

$\mathrm{I}=\mathrm{QMAX}-1$.

$\mathrm{XI}=\mathrm{I}$

$I=10 \div \div(I-3)$

CALL INTNO(I, $.95,0.10)$

$\mathrm{Y} 1=\mathrm{YMIN}$

CALL SOLID $(X 1, Y 1, X I, 0)$

C

CALI MESSAG(23HQUALITY OF GROUND WATER, 23, 7.45, 1.8)

CALL MESSAG(23HIN MILLIGRAMS PER LITER, 23,7.45, 1.5)

CALL MESSAG(21HIS INDICATED BY SHAPE, 21,7.65, 1.2)

CALL MESSAG(19HAND COLOR OF SYMBOL , 19,7.8,0.9)

CALL SETCLR (4HCYAN)

$\mathrm{X} 1=\mathrm{XMAX}-.17 * \mathrm{XINC}$

$\mathrm{Y} 1=\mathrm{YMIN}+.10 * \mathrm{YINC}$

CALL SOLID (X1,Y1,QPLOT, ISYM(1))

WRITE (IDUM, 1000) QWL1

1000 FORMAT $(1 \mathrm{H}<, \mathrm{F} 5.0)$

CALL MESSAG (ITEMP , 6, 8.5, 0.6)

CALL SETCLR (7HMAGENTA)

$\mathrm{Y} 1=\mathrm{YMIN}+.055 * \mathrm{YINC}$

CALL SOLID (X1, Y1, QPLOT, ISYM(2))

WRITE (IDUM, 1010) QWL1, QWL2

1010 FORMAT (F5.0,3H - ,F5.0)

CALL MESSAG(ITEMP , 13,8.5,0.3)

CALL SETCLR(6HYELLOW)

$\mathrm{Y} 1=\mathrm{YMIN}+.01 * \mathrm{YINC}$

CALL SOLID (X1, Y1, QPLOT, ISYM(3))

WRITE (IDUM, 1010) QWL2, QWL3

CALL MESSAG(ITEMP , 13,8.5,0.0)

CALL SETCLR (3HRED)

$\mathrm{Y} 1=$ YMIN $-.035 *$ YINC

CALL SOLID (X1, Y1, QPLOT, ISYM(4))

WRITE (IDUM, 1020) QWL3

1020 FORMAT $(1 \mathrm{H}>, \mathrm{F} 5.0)$

CALL MESSAG(ITEMP , 6,8.5, - .3)

$\mathrm{C}$

C

$\mathrm{C}$

\section{PLOT THE DATA}

DO 60 INODE $=1$, NNODE

IUP = NODEUS (INODE)

IF (IUP .EQ. 0) GO TO 50

$\mathrm{XS} 1=(\mathrm{XLOC}(\mathrm{INODE})+\mathrm{XLOC}($ IUP $)) / 2 .-(\mathrm{YLOC}($ INODE $)-\mathrm{YLOC}($ IUP $)) / 3$.

YS1 $=($ YLOC $($ INODE $)+$ YLOC (IUP $)) / 2+(X L O C($ INODE $)-X L O C(I U P)) / 3$.

$\mathrm{XS} 2=(\mathrm{XLOC}(\mathrm{INODE})+\mathrm{XLOC}(\mathrm{IUP})) / 2+(\mathrm{YLOC}(\mathrm{INODE})-\mathrm{YLOC}(\mathrm{IUP})) / 3$.

YS2 $=($ YLOC $(I N O D E)+Y L O C(I U P)) / 2 .-(X L O C($ INODE $)-X L O C(I U P)) / 3$. 
Attachment 8--FORTRAN Source Code of Subroutines for Water-Supply Operations Simulation--Continued

3836

3837

3838

3839

3840

3841

3842

3843

3844

3845

3846

3847

3848

3849

3850

3851

3852

3853

3854

3855

3856

3857

3858

3859

3860

3861

3862

3863

3864

3865

3866

3867

3868

3869

3870

3871

3872

3873

3874

3875

3876

3877

3878

3879

3880

3881

3882

3883

3884

3885

3886

3887

3888

3889

3890

3891

C

10 IF (GWSTOR(INODE, 1) .LE. 1000.) GO TO 30

$\mathrm{X} 1=\mathrm{XPOSN}(\mathrm{XS} 1, \mathrm{YS} 1)-.35$

$\mathrm{Y} 1=\mathrm{YPOSN}(\mathrm{XS} 1, \mathrm{YS} 1)-.3$

$\mathrm{JQ}=$ GWSTOR $($ INODE, 1$) / 1000$.

CALL SETCLR(5HWHITE)

CALL INTNO(JQ, X1, Y1)

IPEN $=1$

CALL SETCLR(4HCYAN)

IF (GWCONC(INODE, 1) .IT. QWL1) GO TO 20

IPEN $=2$

CALL SETCLR (7HMAGENTA)

IF (GWCONC (INODE, 1) . LT. QWL2) GO TO 20

IPEN $=3$

CALL SETCLR (6HYELLOW)

IF (GWCONC(INODE, 1) . IT. QWL3) GO TO 20

IPEN $=4$

CALL SETCLR (3HRED)

20 CONTINUE

FAC $=$ ALOG 10 (GWSTOR $($ INODE, 1$))$

C

CALI SOLID (XS1, YS1, FAC, ISYM(IPEN))

30 IF (GWSTOR(INODE, 2) .LE. 500.) GO TO 60

$\mathrm{X} 1=\mathrm{XPOSN}(\mathrm{XS} 2, \mathrm{YS} 2)+.2$

$\mathrm{Y} 1=\mathrm{YPOSN}(\mathrm{XS} 2, \mathrm{YS} 2)+.15$

$\mathrm{JQ}=$ GWSTOR $($ INODE, 2$) / 1000$.

CALL SETCLR (5HWHITE)

CALL INTNO(JQ, X1, Y1)

IPEN $=1$

CALL SETCLR(4HCYAN)

IF (GWCONC (INODE, 2) .LT. QWL1) GO TO 40

IPEN $=2$

CALL SETCLR (7HMAGENTA)

IF (GWCONC (INODE, 2) .LT. QWL2) GO TO 40

IPEN $=3$

CALI SETCLR (6HYELLOW)

IF (GWCONC(INODE, 2) .LT. QWL3) GO TO 40

IPEN $=4$

CALL SETCLR(3HRED)

40 CONTINUE

FAC $=$ ALOG 10 (GWSTOR (INODE , 2) $)$

CALL SOLID (XS2, YS2, FAC, ISYM(IPEN))

GO TO 60

50 IDOWN $=$ NXTNOD (INODE)

IF (IDOWN .LE. 0) GO TO 60

$\mathrm{XS} 1=(2 . * X L O C($ INODE $)-\mathrm{XLOC}($ IDOWN $)) / 3$.

YS1 $=(2 . *$ YLOC $($ INODE $)-$ YLOC $($ IDOWN $)) / 3$.

$\mathrm{XS} 2=(\mathrm{XLOC}($ INODE $)-2 . * \mathrm{XLOC}($ IDOWN $)) / 3$.

YS2 $=($ YLOC $($ INODE $)-2 . * Y L O C($ IDOWN $)) / 3$.

GO TO 10

60 CONTINUE

CALL ENDPL(0)

QMAX = SAVQMX

C

RETURN

END 
Attachment 8--FORTRAN Source Code of Subroutines

for Water-Supply Operations Simulation--Continued

\begin{tabular}{|c|c|c|}
\hline $\begin{array}{l}3892 \\
3893\end{array}$ & $\begin{array}{l}\text { CSKMU } \\
\mathrm{C}\end{array}$ & \\
\hline 3894 & $\mathrm{C}$ & SUBROUTINE SCHEMATIC OF RIVER BASIN SIMULATION MODEL \\
\hline 3895 & C & WRITTEN BY ALAN BURNS, U.S. GEOLOGICAL SURVEY \\
\hline 3896 & C & WATER RESOURCES DIVISION, LAKEWOOD, CO \\
\hline 3897 & $\mathrm{C}$ & LATEST UPDATE $1 / 26 / 87$ \\
\hline 3898 & C & \\
\hline 3899 & & SUBROUTINE SKMU(ITITLE) \\
\hline 3900 & & COMMON /DEMND/ NUSERS, USERID $(100), \operatorname{XLOCU}(100), \operatorname{YLOCU}(100)$, \\
\hline 3901 & & $1 \quad \operatorname{DMDFAC}(100), \operatorname{USRTYP}(100), \operatorname{NUMUSR}(100), \operatorname{NSOURC}(100)$ \\
\hline 3902 & & RFTYPE (100), RFYAL (100), RFXLOC (100), RFYLOC (100), \\
\hline 3903 & & NODERF $(100), \operatorname{RFDIST}(100), \operatorname{ICODE}(100,15), \operatorname{IPRECP}(100)$ \\
\hline 3904 & & $\operatorname{QUANTY}(100,15), \operatorname{SCXLOC}(100,15), \operatorname{SCYLOC}(100,15)$, \\
\hline 3905 & & $\operatorname{NODESC}(100,15), \operatorname{WANT}(100), \operatorname{NEED}(100), \operatorname{USXPLT}(100)$ \\
\hline 3906 & & USYPLT $(100), \operatorname{ISIDRF}(100), \operatorname{IAKNT}(100), \operatorname{ITIMFC}(100,15)$, \\
\hline 3907 & & TIMFAC $(100,12)$ \\
\hline 3908 & & INTEGER USERID,USRTYP, RFTYPE \\
\hline 3909 & & REAL NUMUSR, NEED \\
\hline 3910 & & COMMON /NTWRK/ XHY (5), YHY (5), XILNG, YLENG , XMIN, YMIN , XINC, YINC, XMAX, \\
\hline 3911 & & YMAX \\
\hline 3912 & & COMMON /SCLFAC/ QMAX,QWL1, QWL2,QWL3 \\
\hline 3913 & & COMMON /SKMTKU/ ISKMGW, ISKMU, UMAX, ADD (100), AGW(100), ARR(100), \\
\hline 3914 & & $1 \quad \operatorname{AIM}(100), \operatorname{APR}(100), \operatorname{ACN}(100), \operatorname{ANEED}(100)$ \\
\hline 3915 & & COMMON /USTATS/ DELIVR (100), RESREL (100), IMPORT (100), PUMPAG (100), \\
\hline 3916 & & $1 \quad \operatorname{APCONC}(100), \operatorname{DIVRZN}(100), \operatorname{PUMPG}(100,2), \operatorname{CANLEK}(100)$, \\
\hline 3917 & & DPPERC (100), TAILWR (100), PRECIP (100) \\
\hline 3918 & & REAL IMPORT \\
\hline 3919 & $\mathrm{C}$ & 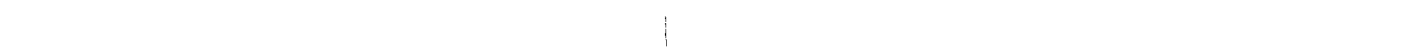 \\
\hline 3920 & & DIMENSION ITITLE (1) \\
\hline 3921 & & DIMENSION ISYM(5), XLINE (5), YLINE (5), ITEMP (20) \\
\hline 3922 & & DIMENSION USEDAT (5) \\
\hline 3923 & & CHARACTER $\div 80$ IDUM \\
\hline 3924 & C & \\
\hline 3925 & & EQUIVALENCE (IDUM, ITEMP) \\
\hline 3926 & C & \\
\hline 3927 & & DATA ISYM $/ 16,1,3,5,17 /$ \\
\hline 3928 & C & \\
\hline 3929 & $\mathrm{C}$ & PLOT THE NETWORK \\
\hline 3930 & C & \\
\hline 3931 & & CALL NETWRK(ITITLE) \\
\hline 3932 & $\mathrm{C}$ & \\
\hline 3933 & C & PLOT THE EXPLANATION \\
\hline 3934 & $\mathrm{C}$ & \\
\hline 3935 & & CALL SETCLR(5HWHITE) \\
\hline 3936 & & CALL MESSAG(17HAPPLIED WATER, IN $, 17,0 ., 2.3$ ) \\
\hline 3937 & & CALL MESSAG( 18 HACRE-FEET PER YEAR , 18,-.05,2.05) \\
\hline 3938 & & CALL MESSAG(15HIS INDICATED BY, $15, .05,1.80)$ \\
\hline 3939 & & CALI MESSAG(23HRELATIVE SIZE OF SYMMOL, $23,-.35,1.55$ ) \\
\hline 3940 & & CALL MESSAG( $17 \mathrm{HDIRECT}$ DIVERSIONS $, 17,1.35, .75)$ \\
\hline 3941 & & CALL MESSAG(20HGROUND-WATER PUMPAGE, $20,-.65,1.00)$ \\
\hline 3942 & & CALL MESSAG(9HRESERVOIR, $9,-.40,0.70$ ) \\
\hline 3943 & & CALL MESSAG( 8 HRELEASES $, 8,-.40, .50)$ \\
\hline 3944 & & CALL MESSAG ( 7 HIMPORTS $, 7, .10, .20)$ \\
\hline
\end{tabular}


Attachment 8--FORTRAN Source Code of Subroutines for Water-Supply Operations Simulation--Continued

3945

3946

3947

3948

3949

3950

3951

3952

3953

3954

3955

3956

3957

3958

3959

3960

3961

3962

3963

3964

3965

3966

3967

3968

3969

3970

3971

3972

3973

3974

3975

3976

3977

3978

3979

3980

3981

3982

3983

3984

3985

3986

3987

3988

3989

3990

3991

3992

3993

3994
CALL MESSAG(13HPRECIPITATION , 13, 1.35, .40)

CALL MESSAG(13HTHE INNER DOT, $13,-.70,-.20$ )

CALL MESSAG(12HIS WATER USE, 12,-.70,-.40)

CALL MESSAG (14HAN OPEN CIRCLE, $14,1.60,-.20$ )

CALL MESSAG(13HIS WATER NEED, $13,1.60,-.40$ )

CALL ENDGR(0)

C

$\mathrm{RAD} 1=.5$

$X=.75+1.0 * X I N C /(X M A X-X M I N)$

$\mathrm{Y}=.5+.7 * \mathrm{YINC} /(\mathrm{YMAX}-\mathrm{YMIN})$

ISAV = ISKMU

ISKMU $=$ NUSERS +1

$\operatorname{USEDAT}(1)=98$.

$\operatorname{ADD}($ ISKMU) $=98$.

$\operatorname{USEDAT}(2)=99$.

$A G W($ ISKMU $)=99$.

$\operatorname{USEDAT}(3)=100$.

$A R R$ (ISKMU) $=100$.

$\operatorname{USEDAT}(4)=101$.

$\operatorname{AIM}($ ISKMU) $=101$.

$\operatorname{USEDAT}(5)=102$.

$A P R(I S K M U)=102$.

C

CALL PHYSOR(X-RAD1/2.,Y-RAD1/2.)

CALL AREA2D (RAD1,RAD1)

CALL HWSHD

CALL PIEPAT (ISYM, 5)

CALL PIEDTA ('NONE')

CALL PIEMOD

CALL SHDPIE $(0,0$, USEDAT, 5$)$

CALL ENDGR(0)

C

$\mathrm{RAD} 1=.2$

CALL PHYSOR(X-RAD1/2., Y-RAD1/2.)

CALL AREA2D (RAD1, RAD1)

CALL RESET ('PIEMOD')

CALL SETCLR (5HWHITE)

CALL PIEPAT $(16,1)$

CALL PIEDTA ('NONE')

CALL SHDPIE $(0,0$, USEDAT, 1$)$

CALL ENDGR (0)

C

$\mathrm{RAD1}=.6$

CALL PHYSOR(X-RAD1/2.,Y-RAD1/2.)

CALL AREA2D (RAD1, RAD1)

CALL RESET ('HWSHD')

CALL PIEPAT $(0,1)$

CALL PIEDTA ('NONE')

CALL $\operatorname{SHDPIE}(0,0$, USEDAT, 1$)$

CALL ENDGR(0) 
3995

3996

3997

3998

3999

4000

4001

4002

4003

4004

4005

4006

4007

4008

4009

4010

4011

4012

4013

4014

4015

4016

4017

4018

4019

4020

4021

4022

4023

4024

4025

4026

4027

4028

4029

4030

4031

4032

4033

4034

4035

4036

4037

4038

4039

4040

4041
C

$\mathrm{RAD} 1=.4$

CALL PHYSOR (X-RAD1/2.,Y-RAD $1 / 2$.

CALL AREA2D (RAD1, RAD1)

CALL SETCLR (5HBLACK)

CALL PIEPAT $(0,1)$

CALL PIEDTA ('NONE')

CALL SHDPIE $(0,0$, USEDAT, 1$)$

CALL ENDGR(0)

C

$\mathrm{C}$

$\mathrm{C}$

ISAV $=$ ISKMU
DO 60 IUSER $=1$, NUSERS

ISKMU = IUSER

TOTAL $=$ ADD $($ IUSER $)+A G W($ IUSER $)+A R R($ IUSER $)+A I M(I U S E R)+$

1 APR (IUSER)

IF (TOTAL .LE. 0.) GO TO 60

IUSE $=1$

IF (ADD (IUSER)/TOTAL .IT. .02) GO TO 10

USEDAT (IUSE) $=$ ADD (IUSER)

IUSE $=$ IUSE +1

10 IF (AGW(IUSER)/TOTAL .LT. .02) GO TO 20

USEDAT (IUSE) $=$ AGW (IUSER)

IUSE $=$ IUSE +1

20 IF (ARR (IUSER)/TOTAL .LT. .02) GO TO 30

USEDAT (IUSE) $=$ ARR (IUSER)

IUSE $=$ IUSE +1

30 IF (AIM(IUSER)/TOTAL .LT. .02) GO TO 40

USEDAT (IUSE) $=$ AIM (IUSER)

IUSE $=$ IUSE +1

40 IF (APR(IUSER)/TOTAL .LT. .02) GO TO 50

USEDAT (IUSE) $=$ APR (IUSER)

IUSE $=$ IUSE +1

50 IUSE $=$ IUSE -1

$X=10 . *(X L O C U($ IUSER $)-X M I N) /($ XMAX-XMIN $)+.75$

$\mathrm{Y}=6.5 *($ YLOCU (IUSER) - YMIN $) /($ YMAX-YMIN $)+.5$

RADI $=1 . /$ ALOG10 (UMAX/TOTAL)

IF (RADI . LT. . 1) GO TO 60

CALL PHYSOR(X-RADI/2.,Y-RADI/2.)

CALL AREA2D (RADI, RADI)

CALL HWSHD

CALL PIEPAT (ISYM, IUSE)

CALL PIEDTA ('NONE')

CALL PIEMOD

CALL SHDPIE $(0,0$, USEDAT , IUSE $)$

CALL ENDGR $(0)$ 
Attachment 8--FORTRAN Source Code of Subroutines for Water-Supply Operations Simulation--Continued

4042

4043

4044

4045

4046

4047

4048

4049

4050

4051

4052

4053

4054

4055

4056

4057

4058

4059

4060

4061

4062

4063

4064

4065

4066

4067

4068

4069

4070

4071

4072
C

RAD1 = RADI*ACN (IUSER)/TOTAL

IF (ACN (IUSER) .LE. 0.) GO TO 60

CALL PHYSOR(X-RAD1/2.,Y-RAD1/2.)

CALL AREA2D (RAD1, RAD1)

CALL RESET ('PIEMOD')

CALL SETCLR (5HWHITE)

CALL PIEPAT $(16,1)$

CALL PIEDTA ('NONE')

$\operatorname{USEDAT}(1)=\operatorname{ACN}($ IUSER)

CALL $\operatorname{SHDPIE}(0,0$, USEDAT, 1$)$

CALL ENDGR (0)

C

RAD1 = RADI*ANEED (IUSER) $/$ TOTAL

IF (ANEED (IUSER) .LE. 0.) GO TO 60

CALL PHYSOR(X-RAD1/2.,Y-RAD1/2.)

CALL AREA2D (RAD1, RAD1)

IF (ANEED(IUSER) . LT. TOTAL ) CALL SETCLR(5HBLACK)

CALL RESET ('HWSHD')

CALL PIEPAT $(0,1)$

CALL PIEDTA ('NONE')

$\operatorname{USEDAT}(1)=\operatorname{ANEED}($ IUSER)

CALL SHDPIE $(0,0$, USEDAT, 1$)$

CALL ENDGR (0)

C

60 CONTINUE

CALL ENDPL (0)

C

ISKMU $=$ ISAV

RETURN

END 
Attachment 8--FORTRAN Source Code of Subroutines

for Water-Supply Operations Simulation--Continued

4073

4074

SUBROUT INE MYPIE (ISEGMT, VALUE, PERCENT , RADIUS, OFFSET , ANGLE, IDRAW,

4075

1 IANNOT)

4076

4077

4078

4079

4080

4081

4082

4083

4084

4085

4086

4087

4088

4089

4090

4091

4092

4093

4094

4095

4096

C

COMMON /SKMTKU/ ISKMGW, ISKMU, UMAX, ADD (100), AGW(100), ARR(100), 1

$\operatorname{AIM}(100), \operatorname{APR}(100), \operatorname{ACN}(100), \operatorname{ANEED}(100)$

C

IF (VALUE .EQ. ADD(ISKMU)) GO TO 10

IF (VALUE .EQ. AGW(ISKMU)) GO TO 20

IF (VALUE .EQ. ARR (ISKMU)) GO TO 30

IF (VALUE .EQ. AIM(ISKMU)) GO TO 40

IF (VALUE .EQ. APR (ISKMU)) GO TO 50

CALL SETCLR (5HWHITE)

RETURN

10 CALL SETCLR(4HCYAN)

RETURN

20 CALL SETCLR(3HRED)

RETURN

30 CALL SETCLR(5HGREEN)

RETURN

40 CALL SETCLR (7HMAGENTA)

RETURN

50 CALL SETCLR (4HBLUE)

RETURN

END 
Attachment 8--FORTRAN Source Code of Subroutines

for Water-Supply Operations Simulation--Continued

\begin{tabular}{|c|c|c|}
\hline $\begin{array}{l}4097 \\
4098\end{array}$ & $\begin{array}{l}\text { CSMRYFU } \\
\mathrm{C}\end{array}$ & \\
\hline 4099 & $\mathrm{C}$ & SUBROUTINE FINAL SUMMARY OF RIVER BASIN SIMULATION MODEL（非） \\
\hline 4100 & $\mathrm{C}$ & WRITTEN BY ALAN BURNS, U.S. GEOLOGICAL SURVEY \\
\hline 4101 & C & WATER RESOURCES DIVISION, LAKEWOOD, CO \\
\hline 4102 & C & LATEST UPDATE $1 / 26 / 87$ \\
\hline 4103 & C & \\
\hline 4104 & & SUBROUTINE SMRYFU \\
\hline 4105 & & COMMON NMONTH, NINDV , IN, IOUT, IPRINT, IGRAPH, IBATCH, IQW, IMONTH, \\
\hline 4106 & & JMONTH, IYEAR \\
\hline 4107 & & COMMON /DEMND/ NUSERS,USERID (100), XLOCU (100), YLOCU $(100)$, \\
\hline 4108 & & DMDFAC $(100), \operatorname{USRTYP}(100), \operatorname{NUMUSR}(100), \operatorname{NSOURC}(100)$ \\
\hline 4109 & & $\operatorname{RFTYPE}(100), \operatorname{RFVAL}(100), \operatorname{RFXLOC}(100), \operatorname{RFYLOC}(100)$, \\
\hline 4110 & & NODERF $(100), \operatorname{RFDIST}(100), \operatorname{ICODE}(100,15), \operatorname{IPRECP}(100)$, \\
\hline 4111 & & QUANTY $(100,15), \operatorname{SCXIOC}(100,15), \operatorname{SCYLOC}(100,15)$, \\
\hline 4112 & & NODESC $(100,15), \operatorname{WANT}(100), \operatorname{NEED}(100), \operatorname{USXPLT}(100)$, \\
\hline 4113 & & USYPLT $(100), \operatorname{ISIDRF}(100), \operatorname{IAKNT}(100), \operatorname{ITIMFC}(100,15)$, \\
\hline 4114 & & $\operatorname{TIMFAC}(100,12)$ \\
\hline 4115 & & INTEGER USERID, USRTYP, RFTYPE \\
\hline 4116 & & REAL NUMUSR, NEED \\
\hline 4117 & & COMMON /DEMND1/ USRNAM (100) \\
\hline 4118 & & DOUBLE PRECISION USRNAM \\
\hline 4119 & & COMMON /HYDGRG/ IHYGW(5), GWHYS $(600,5), \operatorname{GWHYC}(600,5), \operatorname{GWHYRF}(600,5)$ \\
\hline 4120 & & COMMON /HYDGRR/ IHYR(5), RHYS $(600,5)$, RHYC $(600,5)$ \\
\hline 4121 & & COMMON /HYDGRU/ IHYU (5), UHYD $(600,5), \operatorname{UHYP}(600,5), \operatorname{UHYT}(600,5)$ \\
\hline 4122 & & COMMON /NAMES/ TITL (5) \\
\hline 4123 & & COMMON /REACHS/ NNODE, NODEID (100), NXTNOD $(100), \operatorname{XLOC}(100), \operatorname{YLOC}(100)$, \\
\hline 4124 & & $1 \quad \operatorname{PLTX}(100), \operatorname{PLTY}(100), \operatorname{NTRCPT}(100,12), \operatorname{SLOPE}(100,12)$, \\
\hline 4125 & & QWA $(100,12), Q W B(100,12), \operatorname{INDEPV}(100,12), Q(100)$ \\
\hline 4126 & & QW $(100), \operatorname{CONCFC}(100), \operatorname{NODEUS}(100), \mathrm{QSUBA}(100)$ \\
\hline 4127 & & QWSUBA $(100)$, DSA $(100)$, DSB $(100)$ \\
\hline 4128 & & REAL NTRCPT \\
\hline 4129 & & COMMON /REACH1/ NODNAM(100) \\
\hline 4130 & & DOUBLE PRECISION NODNAM \\
\hline 4131 & & COMMON /RESVOR/ NRES, RESSTR(25), RELEAS (25), NODRES (25), RESID (25), \\
\hline 4132 & & CAPMAX (25), NACTIV (25), AREAMX (25), RSXIOC (25), \\
\hline 4133 & & $\operatorname{RSYLOC}(25), \operatorname{RSCONC}(25), \operatorname{RESEVP}(25), \operatorname{RSXPLT}(25)$ \\
\hline 4134 & & $\operatorname{RSYPLT}(25), \operatorname{RESCUM}(25), \operatorname{SBSTOR}(25,5)$ \\
\hline 4135 & & INTEGER RESID \\
\hline 4136 & & REAL NACTIV \\
\hline 4137 & & COMMON /RES1/ RESNAM(25) \\
\hline 4138 & & DOUBLE PRECISION RESNAM \\
\hline 4139 & & COMMON /SKMTKU/ ISKMGW,ISKMU,UMAX,ADD (100), AGW(100), ARR (100), \\
\hline 4140 & & $\operatorname{AIM}(100), \operatorname{APR}(100), \operatorname{ACN}(100), \operatorname{ANEED}(100)$ \\
\hline 4141 & & COMMON /STATR/ ISSR $(10), \operatorname{RMEANS}(10,12), \operatorname{RMEANC}(10,12), \operatorname{RMEANR}(10,12)$, \\
\hline 4142 & & $1 \quad \operatorname{RMEAND}(10,12), \operatorname{RMEANI}(10,12), \operatorname{RMEANE}(10,12)$ \\
\hline 4143 & & COMMON /STATU/ ISSU (10), UMENND $(10,12)$, UMENDD $(10,12), \operatorname{UMENGW}(10,12)$, \\
\hline 4144 & & $1 \quad \operatorname{UMENRR}(10,12), \operatorname{UMENTA}(10,12), \operatorname{UMENP}(10,12)$, \\
\hline 4145 & & UMENWC $(10,12)$, UMENC $(10,12)$, UMENCL $(10,12)$, \\
\hline 4146 & & $\operatorname{UMENTW}(10,12), \operatorname{UMENDP}(10,12)$ \\
\hline 4147 & & COMMON /TABLEG/ ITBLG $(5), \operatorname{IgTBLS}(5,600), \operatorname{IGTBLC}(5,600), \operatorname{IGTBLR}(5,600)$ \\
\hline 4148 & & COMMON /TABLER/ ITBLR (5), IRTBLS $(5,600), \operatorname{IRTBLC}(5,600)$ \\
\hline 4149 & & COMMON /TABLEU/ ITBLU (5), IUTBLD $(5,600), \operatorname{IUTBLP}(5,600), \operatorname{IUTBLT}(5,600)$ \\
\hline
\end{tabular}


Attachment 8--FORTRAN Source Code of Subroutines

for Water-Supply Operations Simulation--Continued

4150

4151

4152

4153

4154

4155

4156

4157

4158

4159

4160

4161

4162

4163

4164

4165

4166

4167

4168

4169

4170

4171

4172

4173

4174

4175

4176

4177

4178

4179

4180

4181

4182

4183

4184

4185

4186

4187

4188

4189

4190

4191

4192

4193

4194

4195

4196

4197

4198

4199

4200
C

REAL ITITLE

EQUIVALENCE (IDUM, ITITLE)

C

$\mathrm{XN}=\mathrm{NMONTH}$

NYEAR $=$ NMONTH $/ 12$

XNYEAR = NYEAR

$\mathrm{C}$

$\mathrm{C}$

$\mathrm{C}$

IF ISKMGW $=99999$, PLOT FINAL GROUND-WATER STORAGE SCHEMATIC

IF (ISKMGW - 99999) 30,10,30

10 IF (IBATCH .EQ. 0) GO TO 20

WRITE (IOUT, 1000)

1000 FORMAT (46H ARE YOU READY FOR THE GROUND-WATER SCHEMATIC?)

CALL QUESZN(IQ)

IF (IQ- 1$) 30,20,30$

20 WRITE (IDUM, 1010) TITL

1010 FORMAT $(5 A 4,17 \mathrm{H}$ - FINAL STORAGE\$ )

CALL SKMGW(ITITLE)

C

C PLOT GROUND-WATER HYDROGRAPHS

C

30 IF (IBATCH .EQ. 0) GO TO 60

DO $50 \mathrm{I}=1,5$

IF (IHYGW(I)) $40,50,40$

40 WRITE (IOUT, 1020)

1020 FORMAT (48H ARE YOU READY FOR THE GROUND-WATER HYDROGRAPHS?)

CALL QUESZN(IQ)

IF (IQ-1) $110,60,110$

50 CONTINUE

GO TO 110

60 DO $100 \mathrm{I}=1,5$

INODE = IHYGW(I)

IF (INODE) $70,100,70$

70 IF (INODE .EQ. 99999) GO TO 80

ISIDE $=$ INODE $/ 1000$

INODE = INODE - ISIDE $\div 1000$

WRITE (IDUM, 1030) TITL, NODEID(INODE), NODNAM(INODE), ISIDE

1030 FORMAT $(5 \mathrm{~A} 4, \mathrm{I} 4,1 \mathrm{H}(, \mathrm{A} 8,7 \mathrm{H}), \mathrm{SIDE}, \mathrm{I} 2,1 \mathrm{HS})$

GO TO 90

80 WRITE (IDUM, 1040) TITL

1040 FORMAT (5A4,32H - BASINWIDE (UNITS ARE 1000'S)\$)

90 CALL HYDRGR(GWHYS $(1, \mathrm{I})$, ITITLE, 7$)$

IF (INODE .EQ. 99999) WRITE (IDUM, 1050) TITL

1050 FORMAT (5A4,13H - BASINWIDE\$)

CALL HYDRGR (GWHYC $(1, I)$, ITITLE, 2)

CALL HYDRGR(GWHYRF $(1, I)$, ITITLE, 8 )

100 CONTINUE 
Attachment 8--FORTRAN Source Code of Subroutines

for Water-Supply Operations Simulation--Continued

4201

4202

4203

4204

4205

4206

4207

4208

4209

4210

4211

4212

4213

4214

4215

4216

4217

4218

4219

4220

4221

4222

4223

4224

4225

4226

4227

4228

4229

4230

4231

4232

4233

4234

4235

4236

4237

4238

4239

4240

4241

4242

4243

4244

4245

4246

4247

4248

4249

4250

4251

4252

C

C LIST GROUND-WATER TABLES

C

110 DO $220 \mathrm{I}=1,5$

INODE $=$ ITBLG(I)

IF (INODE) $120,220,120$

$\mathrm{C}$

120 IF (INODE .EQ. 99999) GO TO 130

ISIDE $=$ INODE $/ 1000$

INODE = INODE - ISIDE $* 1000$

130 DO 210 ITB $=1,3$

IF (IBATCH .EQ. 0) GO TO 140

WRITE (IOUT, 1060)

1060 FORMAT $(/ / / /, 37 \mathrm{H}$ IS YOUR TERMINAL READY FOR THE TABLE)

CALL QUESZN(IQ)

IF (IQ - 1) $210,140,210$

C

140 IF (ITB - 2) $150,170,190$

150 IF (INODE .NE. 99999) WRITE (IOUT, 1070) NODEID(INODE), 1 NODNAM (INODE) , ISIDE , $(\mathrm{J}, \mathrm{J}=1,12)$

1070 FORMAT $(/ / / /, 45 \mathrm{H}$ GROUND-WATER STORAGE, IN ACRE-FEET, FOR NODE ,I5, $12 \mathrm{H}(, \mathrm{A} 8,7 \mathrm{H}), \mathrm{SIDE}, \mathrm{I} 2, / /, 6 \mathrm{H} \mathrm{MONTH}, \mathrm{I} 3,11 \mathrm{I} 6, /, 5 \mathrm{H}$ YEAR $)$

IF (INODE .EQ. 99999) WRITE (IOUT, 1080) (J,J=1,12)

1080 FORMAT (////,56H BASINWIDE GROUND-WATER STORAGE, IN 1000'S OF ACRE 1-FEET ,//, 6H MONTH, I3,1116,/,5H YEAR)

DO 160 IYEAR $=1$, NYEAR

JMONTH $=($ IYEAR -1$) * 12+1$

$\mathrm{KMONTH}=\mathrm{IYEAR} \div 12$

160 WRITE (IOUT, 1090) IYEAR, (IGTBLS (I , IMONTH), IMONTH=JMONTH, KMONTH)

1090 FORMAT $(14, \mathrm{I} 7,1116)$

GO TO 210

C

170 IF (INODE .NE. 99999) WRITE (IOUT, 1100) NODEID(INODE),

1 NODNAM (INODE), ISIDE, ( $\mathrm{J}, \mathrm{J}=1,12)$

1100 FORMAT (////,52H GROUND-WATER CONCENTRATION, IN MILLIGRAMS PER LIT 1ER ,/,5X, 8HFOR NODE, I5, 2H (,A8,7H), SIDE, I2 ,//,6H MONTH, I3, 1116,/, 2 5H YEAR )

IF (INODE .EQ. 99999) WRITE (IOUT, 1110) ( $, \mathrm{J}=1,12)$

1110 FORMAT (////,62H BASINWIDE GROUND-WATER CONCENTRATION, IN MILLIGRA

IMS PER LITER ,//, 6H MONTH, I3,1116,/,5H YEAR)

DO 180 IYEAR $=1$, NYEAR

JMONTH $=($ IYEAR -1$) * 12+1$

$\mathrm{KMONTH}=\operatorname{IYEAR} * 12$

180 WRITE (IOUT, 1090) IYEAR, (IGTBLC ( I , IMONTH) , IMONTH=JMONTH, KMONTH)

GO TO 210

C

190 IF (INODE .NE. 99999) WRITE (IOUT, 1120) NODEID(INODE),

1 NODNAM (INODE), ISIDE , $(J, J=1,12)$

1120 FORMAT ( $/ / / /, 52 \mathrm{H}$ GROUND-WATER RETURN FLOWS, IN CUBIC FEET PER SECO 1ND ,/,5X,8HFOR NODE, I5,2H (,A8,7H), SIDE, I2,//,6H MONTH, I3, $11 \mathrm{I} 6, /$, $25 \mathrm{H}$ YEAR )

IF (INODE .EQ. 99999) WRITE (IOUT, 1130) ( $\mathrm{J}, \mathrm{J}=1,12)$ 
4253

4254

4255

4256

4257

4258

4259

4260

4261

4262

4263

4264

4265

4266

4267

4268

4269

4270

4271

4272

4273

4274

4275

4276

4277

4278

4279

4280

4281

4282

4283

4284

4285

4286

4287

4288

4289

4290

4291

4292

4293

4294

4295

4296

4297

4298

4299

4300

4301

4302

4303

4304

1130 FORMAT (////,62H BASINWIDE GROUND-WATER RETURN FLOWS, IN CUBIC FEE 1T PER SECOND ,//, 6H MONTH, I3,11I6,/,5H YEAR)

DO 200 IYEAR $=1$, NYEAR

JMONTH $=($ IYEAR -1$) * 12+1$

KMONTH $=$ IYEAR $* 12$

200 WRITE (IOUT, 1090) IYEAR, (IGTBLR (I, IMONTH), IMONTH=JMONTH, KMONTH)

210 CONTINUE

220 CONTINUE

$\mathrm{C}$

C IF ISKMU $=99999$, PLOT PERIOD OF RECORD WATER USE SCHEMATIC

$\mathrm{C}$

IF (ISKMU - 99999) $260,230,260$

230 IF (IBATCH .EQ. 0) GO TO 240

WRITE (IOUT, 1140)

1140 FORMAT (43H ARE YOU READY FOR THE WATER USE SCHEMATIC?)

CALL QUESZN(IQ)

IF (IQ-1) $260,240,260$

240 WRITE (IDUM, 1150) TITL

1150 FORMAT $(5 A 4,21 \mathrm{H}$ - AVERAGE WATER USE\$)

DO 250 IUSER $=1$, NUSERS

$\operatorname{ADD}($ IUSER $)=\operatorname{ADD}($ IUSER $) / X N Y E A R$

$\operatorname{AGW}($ IUSER $)=A G W($ IUSER $) / X N Y E A R$

$\operatorname{ARR}($ IUSER $)=A R R($ IUSER) $/$ XNYEAR

$\operatorname{AIM}($ IUSER $)=\operatorname{AIM}($ IUSER $) /$ XNYEAR

$\operatorname{APR}($ IUSER $)=\operatorname{APR}($ IUSER $) /$ XNYEAR

ANEED (IUSER) $=$ ANEED (IUSER) $/$ XNYEAR

C

$250 \mathrm{ACN}($ IUSER $)=A C N($ IUSER $) / X N Y E A R$

CALL SKMU(ITITLE)

C

C PLOT WATER USE HYDROGRAPHS

C

260 IF (IBATCH .EQ. 0) GO TO 290

DO 280 I $=1,5$

IF (IHYU(I)) 270,280,270

270 WRITE (IOUT, 1160)

1160 FORMAT (45H ARE YOU READY FOR THE WATER USE HYDROGRAPHS?)

CALL QUESZN(IQ)

IF (IQ-1) $320,290,320$

280 CONTINUE

GO TO 320

290 DO $310 \mathrm{I}=1,5$

IUSER = IHYU(I)

IF (IUSER) $300,310,300$

300 IF (IUSER . NE. 99999) WRITE (IDUM, 1170) TITL, USERID(IUSER), 1 USRNAM(IUSER)

1170 FORMAT $(5 \mathrm{~A} 4,3 \mathrm{H}-, \mathrm{I} 5,2 \mathrm{H}(, \mathrm{A} 8,2 \mathrm{H}) \$)$

IF (IUSER .EQ. 99999) WRITE (IDUM, 1040) TITL

CALL HYDRGR (UHYD (1, I), ITITLE, 4)

CALL HYDRGR(UHYP $(1, \mathrm{I})$, ITITLE, 5$)$

CALL HYDRGR (UHYT $(1$, I), ITITLE, 6$)$

310 CONTINUE 
Attachment 8--FORTRAN Source Code of Subroutines

for Water-Supply Operations Simulation--Continued

4305

4306

4307

4308

4309

4310

4311

4312

4313

4314

4315

4316

4317

4318

4319

4320

4321

4322

4323

4324

4325

4326

4327

4328

4329

4330

4331

4332

4333

4334

4335

4336

4337

4338

4339

4340

4341

4342

4343

4344

4345

4346

4347

4348

4349

4350

4351

4352

4353

4354

4355

4356
C

C LIST WATER USE TABLES

$\mathrm{C}$

320 DO $420 I=1,5$

IUSER $=$ ITBLU $(\mathrm{I})$

IF (IUSER) $330,420,330$

C

330 DO 410 ITB $=1,3$

IF (IBATCH .EQ. 0) GO TO 340

WRITE (IOUT, 1060)

CALL QUESZN(IQ)

IF (IQ - 1) $410,340,410$

340 IF (ITB - 2) $350,370,390$

350 IF (IUSER . NE. 99999) WRITE (IOUT, 1180) USERID(IUSER),

1 USRNAM ( IUSER) , $(\mathrm{J}, \mathrm{J}=1,12)$

1180 FORMAT (////,42H DIRECT DIVERSIONS, IN ACRE-FEET, FOR USER , I5,

$12 \mathrm{H}(, \mathrm{A} 8,1 \mathrm{H}), / /, 6 \mathrm{H}$ MONTH, $\mathrm{I} 3,11 \mathrm{I} 6, /, 5 \mathrm{H}$ YEAR $)$

IF (IUSER .EQ. 99999) WRITE (IOUT, 1190) ( $J, J=1,12)$

1190 FORMAT (////,52H BASINWIDE DIRECT DIVERSIONS, IN 1000'S OF ACRE-FE

1ET,$/ /, 6 \mathrm{H}$ MONTH, I3, $1116, /, 5 \mathrm{H}$ YEAR)

DO 360 IYEAR $=1$, NYEAR

JMONTH $=($ IYEAR -1$) * 12+1$

KMONTH $=$ IYEAR $* 12$

360 WRITE (IOUT, 1090) IYEAR, (IUTBLD ( I , IMONTH), IMONTH=JMONTH, KMONTH) GO TO 410

C

370 IF (IUSER . NE. 99999) WRITE (IOUT, 1200) USERID(IUSER), 1 USRNAM (IUSER) , $(\mathrm{J}, \mathrm{J}=1,12$ )

1200 FORMAT $(/ / / /, 46 \mathrm{H}$ GROUND-WATER PUMPAGE, IN ACRE-FEET, FOR USER , I5, $12 \mathrm{H}(, \mathrm{A} 8,1 \mathrm{H}), / /, 6 \mathrm{H}$ MONTH, I3,11I6,/,5H YEAR)

IF (IUSER .EQ. 99999) WRITE (IOUT, 1210) ( $, \mathrm{J}=1,12)$

1210 FORMAT (////,55H BASINWIDE GROUND-WATER PUMPAGE, IN 1000'S OF ACRE 1-FEET , //, 6H MONTH, I3,11I6,/,5H YEAR)

DO 380 IYEAR $=1$, NYEAR

JMONTH $=($ IYEAR -1$) \div 12+1$

KMONTH $=$ IYEAR $* 12$

380 WRITE (IOUT, 1090) IYEAR, (IUTBLP ( I , IMONTH), IMONTH=JMONTH, KMONTH)

GO TO 410

C

390 IF (IUSER .NE. 99999) WRITE (IOUT, 1220) USERID(IUSER),

1 USRNAM (IUSER), $(\mathrm{J}, \mathrm{J}=1,12$ )

1220 FORMAT $(/ / / /, 43 \mathrm{H}$ TOTAL APPLICATIONS, IN ACRE-FEET, FOR USER, I5,

$12 \mathrm{H}(\mathrm{A} 8,1 \mathrm{H}), / /, 6 \mathrm{H}$ MONTH, I $3,11 \mathrm{I} 6, /, 5 \mathrm{H}$ YEAR)

IF (IUSER . EQ. 99999) WRITE (IOUT, 1230) (J, J=1, 12)

1230 FORMAT $\left(/ / / /, 52 \mathrm{H}\right.$ BASINWIDE TOTAL APPLICATIONS, IN $1000^{\prime} \mathrm{S}$ OF ACRE-FE

1ET , //,6H MONTH, I3, 11I6,/,5H YEAR)

DO 400 IYEAR $=1$, NYEAR

JMONTH $=($ IYEAR -1$) * 12+1$

KMONTH $=$ IYEAR $* 12$

400 WRITE (IOUT, 1090) IYEAR, (IUTBLT (I , IMONTH), IMONTH=JMONTH, KMONTH)

410 CONT INUE

420 CONT INUE 
Attachment 8--FORTRAN Source Code of Subroutines

for Water-Supply Operations Simulation--Continued

\begin{tabular}{|c|c|c|c|}
\hline 4357 & \multirow{2}{*}{\multicolumn{3}{|c|}{ LIST WATER USE STATISTICS }} \\
\hline 4358 & & & \\
\hline 4359 & C & & \\
\hline 4360 & & $1004801=1,10$ & \\
\hline 4361 & & IUSER $=$ ISSU $(\mathrm{I})$ & \\
\hline 4362 & & IF (IUSER) $430,480,430$ & \\
\hline 4363 & 430 & IF (IBATCH .EQ. 0) GO TO 440 & \\
\hline 4364 & & WRITE (IOUT, 1240) & \\
\hline 4365 & 1240 & FORMAT $(/ / / /, 42 \mathrm{H}$ IS YOUR TERMINAL & READY FOR THE STATISTICS ) \\
\hline 4366 & & CALL QUESZN(IQ) & \\
\hline 4367 & & IF $(I Q-1) 480,440,480$ & \\
\hline 4368 & 440 & IF (IUSER .NE. 99999) GO TO 450 & \\
\hline 4369 & & WRITE (IOUT, 1250) & \\
\hline 4370 & 1250 & FORMAT $(/ / / /, 53 \mathrm{H}$ THE MONTHLY STAT] & ISTICS FOR TOTAL BASINWIDE WATER \\
\hline 4371 & & 1USE,$/, 80 \mathrm{H}$ [VALUES ARE $1000 \mathrm{ACRE}$ & E-FEET EXCEPT CONCENTRATION WHICH \\
\hline 4372 & & 2 IS MILLIGRAMS PER LITER],$/ /, 781$ & SH MONTH WATER DIRECT GROUND RESER \\
\hline 4373 & & 3- TOTAL PRE- WATER CON- CANAL & TAIL DEEP ,/, 78H \\
\hline 4374 & & 4 DIVER- WATER VOIR WATER CIP- & - CON- CEN- LEAKAGE WATER PER- \\
\hline 4375 & & $5, /, 14 X, 44 \mathrm{HSIONS}$ PUMP- RE- & AP- ITA- SUMED TRA- , 16X, \\
\hline 4376 & & 6 5HCOLA- ,/, 21X,37HAGE LEASES & PLIED TION TION, $16 \mathrm{X}$, \\
\hline 4377 & & 7 4HTION ) & \\
\hline 4378 & & GO TO 460 & \\
\hline 4379 & 450 & WRITE (IOUT, 1260) USERID(IUSER), US & ISRNAM (IUSER) \\
\hline 4380 & 1260 & FORMAT $(/ / / /, 33 \mathrm{H}$ THE MONTHLY STAT & ISTICS FOR USER , I5,2H (,A8,1H), \\
\hline 4381 & & $/, 75 \mathrm{H}$ [VALUES ARE ACRE-FEE] & T EXCEPT CONCENTRATION WHICH IS M \\
\hline 4382 & & 2ILLIGRAMS PER LITER] ,//, & BH MONTH WATER DIRECT GROUND RESER \\
\hline 4383 & & 3- TOTAL PRE- WATER CON- CANAL & TAIL DEEP,$/, 78 \mathrm{H}$ \\
\hline 4384 & & 4 DIVER- WATER VOIR WATER CIP- & CON- CEN- LEAKAGE WATER PER- \\
\hline 4385 & & $5, /, 14 \mathrm{X}, 44 \mathrm{HSIONS}$ PUMP- RE- & AP- $\quad$ ITA- SUMED TRA- , 16X, \\
\hline 4386 & & 6 5HCOLA- $, /, 21 \mathrm{X}, 37 \mathrm{HAGE}$ LEASES & PLIED TION TION , 16X, \\
\hline 4387 & & 7 4HTION ) & \\
\hline 4388 & 460 & TUMND $=0$ & \\
\hline 4389 & & TUMDD $=0$ & \\
\hline 4390 & & TUMGW $=0$. & \\
\hline 4391 & & TUMRR $=0$ & \\
\hline 4392 & & TUMTA $=0$ & \\
\hline 4393 & & TUMP $=0$ & \\
\hline 4394 & & TUMWC $=0$. & \\
\hline 4395 & & TUMC $=0$ & \\
\hline 4396 & & TUMCL $=0$. & \\
\hline 4397 & & TUMTW $=0$. & \\
\hline 4398 & & TUMDP $=0$. & \\
\hline 4399 & & DO 470 JMONTH $=1,12$ & \\
\hline 4400 & & TUMND $=$ TUMND + UMENND $(I$, JMONTH $)$ & \\
\hline 4401 & & TUMDD $=$ TUMDD + UMENDD (I, JMONTH $)$ & \\
\hline 4402 & & TUMGW $=$ TUMGW + UMENGW (I, JMONTH $)$ & \\
\hline 4403 & & TUMRR $=$ TUMRR + UMENRR (I, JMONTH $)$ & \\
\hline 4404 & & TUMTA $=$ TUMTA + UMENTA $(I, J M O N T H)$ & \\
\hline 4405 & & TUMP $=$ TUMP + UMENP $(I$, JMONTH $)$ & \\
\hline 4406 & & TUMWC $=$ TUMWC + UMENWC $(I, J M O N T H)$ & \\
\hline 4407 & & TUMC $=$ TUMC $+\operatorname{UMENC}(I, J M O N T H)$ & \\
\hline
\end{tabular}


Attachment 8--FORTRAN Source Code of Subroutines

for Water-Supply Operations Simulation--Continued

4408

4409

4410

4411

4412

4413

4414

4415

4416

4417

4418

4419

4420

4421

4422

4423

4424

4425

4426

4427

4428

4429

4430

4431

4432

4433

4434

4435

4436

4437

4438

4439

4440

4441

4442

4443

4444

4445

4446

4447

4448

4449

4450

4451

4452

4453

4454

4455

4456

4457

4458

4459

4460

4461

4462

TUMCL $=$ TUMCL + UMENCL $(I$, JMONTH $)$

TUMTW $=$ TUMTW $+\operatorname{UMENTW~}(I$, JMONTH $)$

TUMDP $=$ TUMDP + UMENDP $(I$, JMONTH $)$

$\mathrm{XM1}=\operatorname{UMENND}(\mathrm{I}, \mathrm{JMONTH}) / \mathrm{XNYEAR}$

$\mathrm{XM} 2=\operatorname{UMENDD}(\mathrm{I}, \mathrm{JMONTH}) / \mathrm{XNYEAR}$

XM3 = UMENGW(I, JMONTH) $/$ XNYEAR

XM4 = UMENRR ( I , JMONTH) $/$ XNYEAR

XM5 = UMENTA (I, JMONTH) $/$ XNYEAR

XM6 $=\operatorname{UMENP}(I$, JMONTH $) / X N Y E A R$

$\mathrm{XM7}=\operatorname{UMENWC}(\mathrm{I}, \mathrm{JMONTH}) / \mathrm{XNYEAR}$

XM8 $=\operatorname{UMENC}(\mathrm{I}, \mathrm{JMONTH}) / \mathrm{XNYEAR}$

IF (IQW .GT . 0 .AND. (UMENTA ( I , JMONTH) +UMENP ( I , JMONTH)) .NE . 0. )

1 XM8 $=\operatorname{UMENC}(\mathrm{I}, \mathrm{JMONTH}) /($ UMENTA $(\mathrm{I}, \mathrm{JMONTH})+\operatorname{UMENP}(\mathrm{I}, \mathrm{JMONTH}))$

XM9 $=$ UMENCL $(I$, JMONTH) $/$ XNYEAR

XM10 $=\operatorname{UMENTW}(I$, JMONTH $) /$ XNYEAR

XM1 1 = UMENDP $(I$, JMONTH $) / X N Y E A R$

470 WRITE (IOUT, 1270) JMONTH, XM1 ,XM2, XM3 ,XM4 ,XM5 ,XM6 ,XM7 , XM8 , XM9 , XM10,

1 XM11

1270 FORMAT (I $4,1 \mathrm{X}, 5 \mathrm{~F} 7.0, \mathrm{~F} 6.0, \mathrm{~F} 7.0, \mathrm{~F} 6.0,3 \mathrm{~F} 7.0)$

TUMND $=$ TUMND $/$ XNYEAR

TUMDD $=$ TUMDD $/$ XNYEAR

TUMGW $=$ TUMGW $/$ XNYEAR

TUMRR $=$ TUMRR $/$ XNYEAR

TUMTA $=$ TUMTA $/$ XNYEAR

TUMP $=$ TUMP $/$ XNYEAR

TUMWC $=$ TUMWC $/$ XNYEAR

TUMC $=\mathrm{TUMC} / \mathrm{XN}$

IF (IQW .GT. 0 .AND. (TUMTA+TUMP) .NE. 0.)

1 TUMC $=$ TUMC $* X N /(($ TUMTA+TUMP $) * X N Y E A R)$

TUMCL $=$ TUMCL $/$ XNYEAR

TUMTW $=$ TUMTW $/$ XNYEAR

TUMDP $=$ TUMDP $/$ XNYEAR

WRITE (IOUT , 1280) TUMND, TUMDD, TUMGW, TUMRR, TUMTA, TUMP, TUMWC, TUMC,

1 TUMCL, TUMTW, TUMDP

1280 FORMAT $(/$, 5HTOTAL, 5F7.0,F6.0,F7.0,F6.0, 3F7.0)

C

480 CONT INUE

$\mathrm{C}$

C

\section{PLOT RESERVOIR HYDROGRAPHS}

IF (IBATCH .EQ. 0) GO TO 510

DO $500 \mathrm{I}=1,5$

IF (IHYR(I)) $490,500,490$

490 WRITE (IOUT, 1290)

1290 FORMAT (45H ARE YOU READY FOR THE RESERVOIR HYDROGRAPHS?)

CALL QUESZN(IQ)

IF (IQ- 1$) \quad 540,510,540$

500 CONTINUE

GO TO 540

510 DO $530 \mathrm{I}=1,5$

$\operatorname{IRES}=\operatorname{IHYR}(\mathrm{I})$

IF (IRES) $520,530,520$

520 WRITE (IDUM, 1170) TITL, RESID (IRES), RESNAM (IRES)

CALL HYDRGR(RHYS $(1, \mathrm{I}), \operatorname{ITITLE}, 7)$

CALL HYDRGR(RHYC $(1, \mathrm{I})$, ITITLE, 2$)$

530 CONTINUE 
Attachment 8--FORTRAN Source Code of Subroutines

for Water-Supply Operations Simulation--Continued

4463

4464

C

4465

4466

4467

4468

4469

4470

4471

4472

4473

4474

4475

4476

4477

4478

4479

4480

4481

4482

4483

4484

4485

4486

4487

4488

4489

4490

4491

4492

4493

4494

4495

C

C

540 D0 $620 \mathrm{I}=1,5$

IRES $=\operatorname{ITBLR}(\mathrm{I})$

C

IF (IRES) $550,620,550$

550 DO 610 ITB $=1,2$

IF (IBATCH .EQ. 0) GO TO 560

WRITE (IOUT, 1060)

CALL QUESZN(IQ)

IF (IQ - 1) $610,560,610$

560 IF (ITB - 2) $570,590,590$

570 WRITE (IOUT, 1300) RESID (IRES), RESNAM (IRES), ( $\mathrm{J}, \mathrm{J}=1,12$ )

1300 FORMAT $(/ / / /, 38 \mathrm{H}$ CONTENTS, IN ACRE-FEET, FOR RESERVIOR , I5, 2H (,A8 $1,1 \mathrm{H}), / /, 6 \mathrm{H}$ MONTH, I3,1116,/,5H YEAR)

$\mathrm{C}$

DO 580 IYEAR $=1$, NYEAR

JMONTH $=($ IYEAR -1$) * 12+1$

KMONTH $=$ IYEAR $* 12$

580 WRITE (IOUT, 1090) IYEAR, (IRTBLS (I , IMONTH), IMONTH=JMONTH, KMONTH) GO TO 610

590 WRITE (IOUT, 1310) RESID(IRES), RESNAM(IRES), ( $, \mathrm{J}=1,12$ )

1310 FORMAT (////,54H CONCENTRATION, IN MILLIGRAMS PER LITER, FOR RESER 1VOIR , I5, $2 \mathrm{H}(, \mathrm{A} 8,1 \mathrm{H}), / /, 6 \mathrm{H}$ MONTH, I3,11I6,/,5H YEAR)

$\mathrm{C}$

DO 600 IYEAR $=1$, NYEAR

JMONTH $=($ IYEAR -1$) * 12+1$

$\mathrm{KMONTH}=$ IYEAR*12

600 WRITE (IOUT , 1090) IYEAR, (IRTBLC (I , IMONTH), IMONTH=JMONTH, KMONTH)

GO TO 610

610 CONTINUE

620 CONTINUE 
Attachment 8--FORTRAN Source Code of Subroutines

for Water-Supply Operations Simulation--Continued

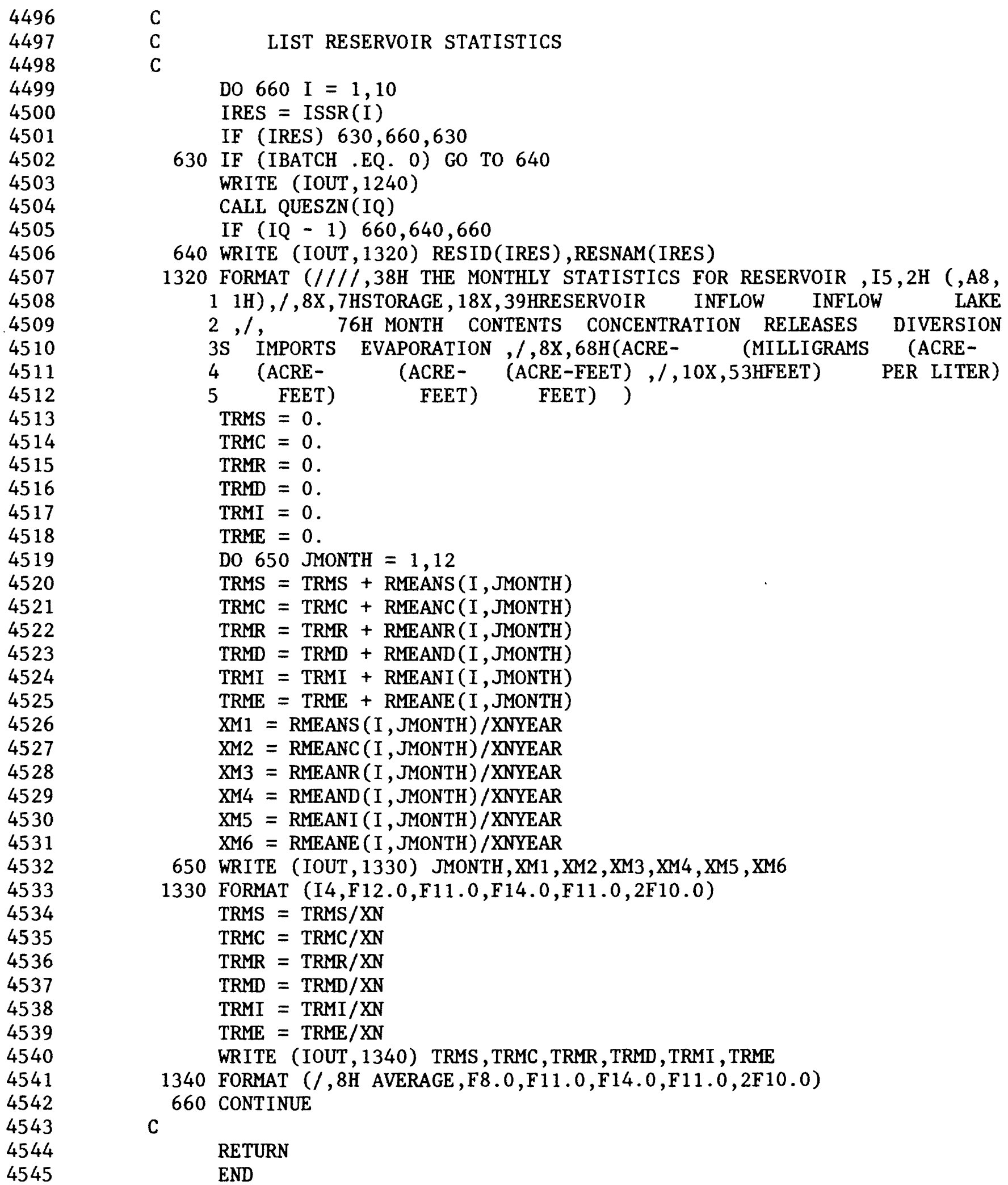


Attachment 8--FORTRAN Source Code of Subroutines

for Water-Supply Operations Simulation--Continued

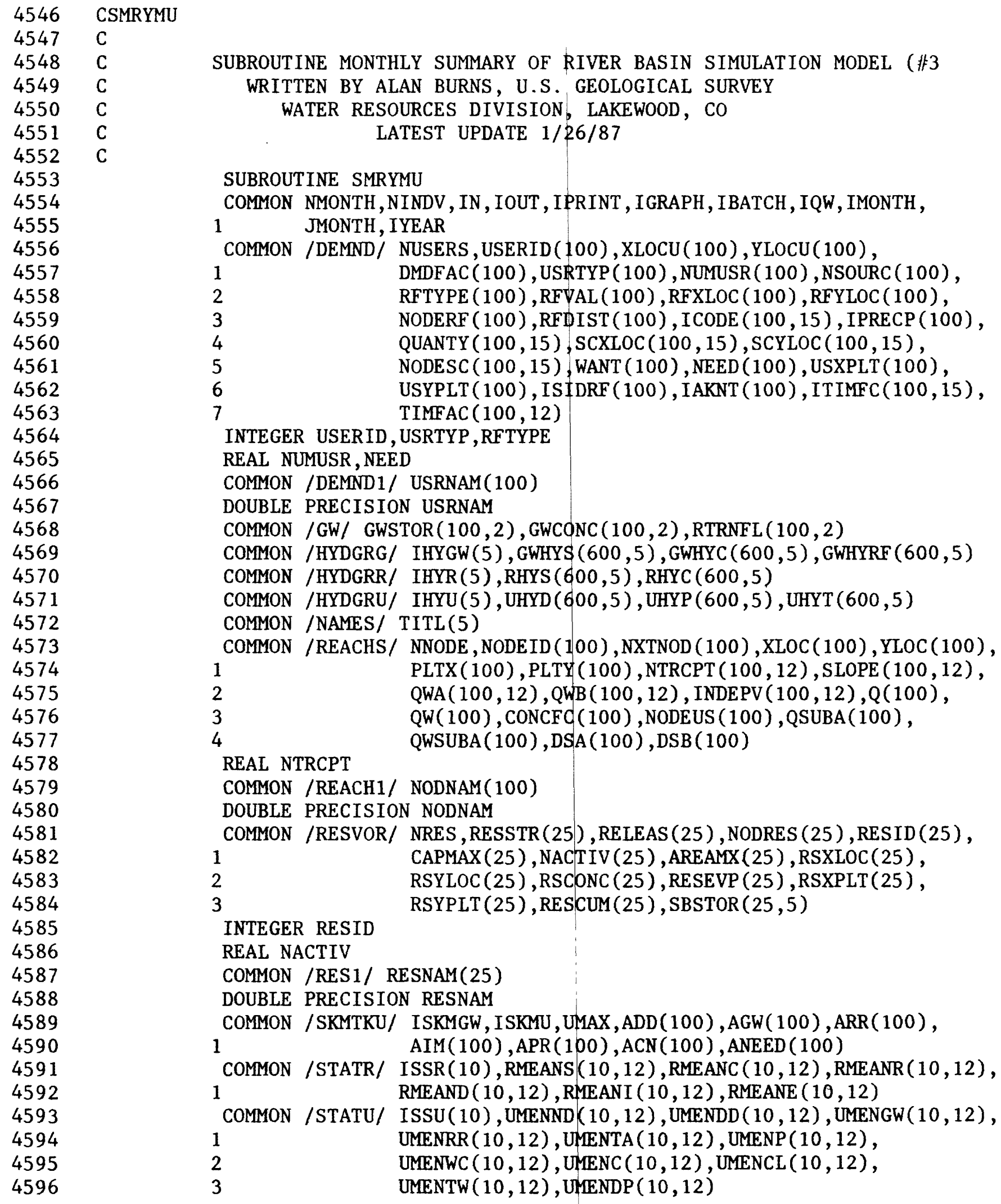


Attachment 8--FORTRAN Source Code of Subroutines

for Water-Supply Operations Simulation--Continued

4597

4598

4599

4600

4601

4602

4603

4604

4605

4606

4607

4608

4609

4610

4611

4612

4613

4614

4615

4616

4617

4618

4619

4620

4621

4622

4623

4624

4625

4626

4627

4628

4629

4630

4631

4632

4633

4634

4635

4636

4637

4638

4639

4640

4641

4642

4643

4644

4645

4646

4647

4648

4649

4650

4651

COMMON /TABLEG/ ITBLG(5), $\operatorname{IGTBLS}(5,600), \operatorname{IGTBLC}(5,600), \operatorname{IGTBLR}(5,600)$

COMMON /TABLER/ ITBLR (5), IRTBLS $(5,600), \operatorname{IRTBLC}(5,600)$

COMMON /TABLEU/ ITBLU(5), IUTBLD $(5,600), \operatorname{IUTBLP}(5,600), \operatorname{IUTBLT}(5,600)$

COMMON /USTATS/ DELIVR(100), RESREL (100), IMPORT (100), PUMPAG (100),

1

2

REAL IMPORT

C

$\mathrm{C}$

$\mathrm{C}$

$\mathrm{C}$

$\mathrm{C}$

WRITE $(54,1000)$ IMONTH

1000 FORMAT (12H1 FOR MONTH , I5, //,21H NODE NODE NODE , 13X, 1 6HSIDE $1,22 \mathrm{X}, 6 \mathrm{HSIDE} 2, /, 76 \mathrm{H}$ NUMBER ID NAME STORAGE CON 2CEN- RETURN STORAGE CONCEN- RETURN ,/,34X,14HTRATION FLOW, 3 13X, 14HTRATION FLOW , /, 25X,51H(ACRE- (MILLI- (CUBIC (ACRE 4- (MILLI- (CUBIC , /, 27X,48HFEET) GRAMS FEET FEET) 5GRAMS FEET ,/, 36X, 12HPER PER , 15X,12HPER PER ,/, 6 35X, 15HLITER) SECOND) ,12X,15HLITER) SECOND) )

DO 10 ISIDE $=1,2$

$\operatorname{TOTST}($ ISIDE) $=0$.

$\operatorname{AVGCN}(I S I D E)=0$.

10 TOTRF (ISIDE) $=0$.

DO 80 INODE $=1$, NNODE

WRITE $(54,1010)$ INODE, NODEID (INODE), NODNAM (INODE),

1 (GWSTOR (INODE, J) , GWCONC (INODE , J), RTRNFL (INODE , J) , J=1, 2)

1010 FORMAT (I6, I7, 2X, A8, 2F9.0,F8.0, 1X, 2F9.0, F8.0)

DO 80 ISIDE $=1,2$

TOTST (ISIDE) $=$ TOTST (ISIDE) + GWSTOR(INODE, ISIDE)

$\operatorname{AVGCN}($ ISIDE $)=$ AVGCN (ISIDE) + GWCONC (INODE, ISIDE)

IF (IQW .GT. 0) AVGCN(ISIDE) = AVGCN(ISIDE)-GWCONC (INODE, ISIDE)+

1 GWCONC (INODE, ISIDE) *GWSTOR (INODE, ISIDE)

TOTRF (ISIDE) $=$ TOTRF (ISIDE) + RTRNFL (INODE, ISIDE)

INOD $=$ INODE + $1000 *$ ISIDE

$\mathrm{C}$

C

STORE HYDROGRAPH DATA

DO $40 \quad I=1,5$

IF (IHYGW(I) .EQ. 99999) GO TO 30

IF (INOD - IHYGW(I) ) $40,20,40$

20 GWHYS (IMONTH, I) = GWSTOR (INODE, ISIDE)

GWHYC (IMONTH, I) = GWCONC (INODE, ISIDE)

GWHYRF (IMONTH, I) $=$ RTRNFL (INODE, ISIDE)

GO TO 40

30 GWHYS (IMONTH, I) = GWHYS (IMONTH, I ) + GWSTOR (INODE, ISIDE) $/ 1000$.

GWHYC (IMONTH, I) = GWHYC (IMONTH, I) + GWCONC (INODE, ISIDE)

IF (IQW . GT. 0) GWHYC (IMONTH, I) $=$ GWHYC (IMONTH, I) -

1 GWCONC (INODE, ISIDE)+GWCONC (INODE, ISIDE) *GWSTOR (INODE, ISIDE) $/ 1000$.

GWHYRF (IMONTH, I) = GWHYRF (IMONTH, I ) + RTRNFL (INODE, ISIDE)

40 CONTINUE 
Attachment 8--FORTRAN Source Code of Subroutines

for Water-Supply Operations Simulation--Continued

4652

4653

4654

4655

4656

4657

4658

4659

4660

4661

4662

4663

4664

4665

4666

4667

4668

4669

4670

4671

4672

4673

4674

4675

4676

4677

4678

4679

4680

4681

4682

4683

4684

4685

4686

4687

4688

4689

4690

4691

4692

4693

4694
STORE TABLE DATA

C

C

DO $70 \quad I=1,5$

IF (ITBLG(I) .EQ. 99999) GO TO 60

IF (INOD - ITBLG(I)) $70,50,70$

50 IGTBLS ( I, IMONTH) $=$ GWSTOR (INODE, ISIDE) +.5

IGTBLC (I, IMONTH) $=$ GWCONC (INODE, ISIDE) +.5

$\operatorname{IGTBLR}(I$, IMONTH $)=$ RTRNFL (INODE, ISIDE) +.5

GO TO 70

60 IGTBLS (I, IMONTH) $=$ IGTBLS (I, IMONTH) + GWSTOR (INODE, ISIDE) $/ 1000$.

$\operatorname{IGTBLC}(I, I M O N T H)=\operatorname{IGTBLC}(I$, IMONTH $)+$ GWCONC (INODE, ISIDE)

IF (IQW .GT. 0) IGTBLC (I, IMONTH) $=$ IGTBLC (I, IMONTH) -

1 GWCONC (INODE, ISIDE) +GWCONC (INODE, IS IDE) *GWSTOR (INODE, IS IDE) $/ 1000$.

IGTBLR (I, IMONTH) = IGTBLR (I, IMONTH) + RTRNFL (INODE, ISIDE)

70 CONTINUE

80 CONTINUE

C

IF (IQW .EQ. 0) GO TO 90

IF (TOTST (1) .NE. 0.) $\operatorname{AVGCN}(1)=\operatorname{AVGCN}(1) / \operatorname{TOTST}(1)$

IF (TOTST (2) .NE. 0.) $\operatorname{AVGCN}(2)=\operatorname{AVGCN}(2) / T O T S T(2)$

GO TO 100

$90 \operatorname{AVGCN}(1)=\operatorname{AVGCN}(1) / \mathrm{NNODE}$

$\operatorname{AVGCN}(2)=\operatorname{AVGCN}(2) / \mathrm{NNODE}$

100 DO $120 \mathrm{I}=1,5$

IF (IHYGW(I) .NE. 99999) GO TO 110

IF (IQW .NE . 0) GWHYC (IMONTH, I) $=$ GWHYC (IMONTH, I) $/$ GWHYS (IMONTH, I)

IF (IQW .EQ. 0) GWHYC (IMONTH,I) $=$ GWHYC (IMONTH, I $) /($ NNODE $* 2$.

110 IF (ITBLG(I) .NE. 99999) GO TO 120

IF (IQW .NE. 0) IGTBLC ( I, IMONTH) $=$ IGTBLC ( I , IMONTH)/IGTBLS ( I , IMONTH)

IF (IQW .EQ. 0) IGTBLC (I, IMONTH) $=\operatorname{IGTBLC}(I, I M O N T H) /(N N O D E * 2)$

120 CONTINUE

WRITE $(54,1020) \operatorname{TOTST}(1), \operatorname{AVGCN}(1), \operatorname{TOTRF}(1), \operatorname{TOTST}(2), \operatorname{AVGCN}(2)$,

1 TOTRF (2)

1020 FORMAT (/,6X,5HTOTAL, 12X, 2F9.0, F8.0, 1X, 2F9.0,F8.0)

C

C

C

IF ISKMGW $=12$, PLOT END OF YEAR GROUND-WATER STORAGE SCHEMATIC

IF (ISKMGW - 12) $150,130,150$

130 IF (JMONTH - 12) $150,140,150$

140 WRITE (IDUM, 1030) TITL, IYEAR

1030 FORMAT $(5 A 4,8 \mathrm{H}$ - YEAR , I3,1H\$)

CALL SKMGW(ITITLE) 
Attachment 8--FORTRAN Source Code of Subroutines

for Water-Supply Operations Simulation--Continued

4695

4696

4697

4698

4699

4700

4701

4702

4703

4704

4705

4706

4707

4708

4709

4710

4711

4712

4713

4714

4715

4716

4717

4718

4719

4720

4721

4722

4723

4724

4725

4726

4727

4728

4729

4730

4731

4732

4733

4734

4735

4736

4737

4738

4739

4740

4741

4742

4743

4744

C

C SUMMARIZE WATER USER DATA

C

150 WRITE $(52,1040)$ IMONTH

1040 FORMAT (12H1 FOR MONTH , I5, 15X,84H[ALL VALUES ARE IN ACRE-FEET EXC 1EPT CONCENTRATION WHICH IS IN MILLIGRAMS PER LITER] ,//,

$2127 \mathrm{H}$ USER USER USER WATER DIRECT GROUND RESER- I 3MPORTS TOTAL PRECIP- WATER CONCEN- CANAL TAIL DEEP $4, /, 56 \mathrm{H}$ NUMBER ID NAME NEED DIVER- WATER VOIR , 514X,59HWATER ITATION CONSUMED TRATION LEAKAGE WATER PERCOL$6, /, 34 \mathrm{X}, 24 \mathrm{HSIONS}$ PUMPAGE RELEASES, 11X,7HAPPLIED , 47X,5HATION )

TNEED $=0$.

TDLVR $=0$.

TPUMP $=0$.

TRES $=0$.

TIMPRT $=0$.

TTTL $=0$.

TPRCP $=0$.

TCONSM $=0$.

AVGCON $=0$.

TLEAK $=0$.

TTAIL $=0$.

$\mathrm{TDP}=0$.

DO 260 IUSER $=1$, NUSERS

TOTAL $=0$.

CONSUM $=0$.

TOTAL = DELIVR (IUSER $)+$ PUMPAG (IUSER)+RESREL( IUSER)+IMPORT (IUSER) -

1 CANLEK (IUSER)

IF (USRTYP(IUSER) .GT. 5) GO TO 160

CONSUM = AMIN1 $($ TOTAL+PRECIP (IUSER), NEED (IUSER)

TNEED $=$ TNEED + NEED (IUSER)

TDLVR $=$ TDLVR + DELIVR (IUSER)

TPUMP = TPUMP + PUMPAG (IUSER)

TRES = TRES + RESREL (IUSER)

TIMPRT = TIMPRT + IMPORT (IUSER)

TTTL $=$ TTTL + TOTAL

TPRCP = TPRCP + PRECIP (IUSER)

TCONSM $=$ TCONSM + CONSUM

AVGCON $=$ AVGCON + (TOTAL+PRECIP(IUSER $) *$ APCONC (IUSER)

TLEAK = TLEAK + CANLEK (IUSER)

TTAIL = TTAIL + TAILWR (IUSER)

C

$\mathrm{TDP}=\mathrm{TDP}+\mathrm{DPPERC}($ IUSER$)$

$A D D($ IUSER $)=A D D($ IUSER) + DELIVR (IUSER)

AGW (IUSER) $=$ AGW (IUSER) + PUMPAG (IUSER)

ARR (IUSER) $=$ ARR (IUSER) + RESREL (IUSER)

AIM (IUSER) $=$ AIM (IUSER) + IMPORT (IUSER)

APR (IUSER) $=$ APR (IUSER) + PRECIP (IUSER)

ANEED (IUSER) $=$ ANEED (IUSER) + NEED (IUSER)

$A C N($ IUSER $)=A C N($ IUSER $)+$ CONSUM 
Attachment 8--FORTRAN Source Code of Subroutines

for Water-Supply Operations Simulation--Cont inued

\begin{tabular}{|c|c|c|}
\hline 4745 & $\mathrm{C}$ & \\
\hline 4746 & C & STORE HYDROGRAPH DATA \\
\hline 4747 & $\mathrm{C}$ & \\
\hline 4748 & 160 & DO $190 I=1,5$ \\
\hline 4749 & & IF (IHYU(I) .EQ. 99999) GO TO 180 \\
\hline 4750 & & IF (IUSER - IHYU(I)) $190,170,190$ \\
\hline 4751 & 170 & UHYD $($ IMONTH, I $)=$ DELIVR (IUSER $)$ \\
\hline 4752 & & UHYP (IMONTH, I $)=$ PUMPAG (IUSER) \\
\hline 4753 & & UHYT (IMONTH, I ) = TOTAL \\
\hline 4754 & & GO TO 190 \\
\hline 4755 & 180 & IF (USRTYP(IUSER) .GT. 5) GO TO 190 \\
\hline 4756 & & UHYD $($ IMONTH,$I)=$ UHYD $($ IMONTH,$I)+$ DELIVR $($ IUSER $) / 1000$. \\
\hline 4757 & & UHYP $($ IMONTH, I $)=$ UHYP (IMONTH,,$f)+\operatorname{PUMPAG}($ IUSER $) / 1000$. \\
\hline 4758 & & UHYT (IMONTH, I $)=$ UHYT (IMONTH, I $)+$ TOTAL $/ 1000$ \\
\hline 4759 & 190 & CONTINUE \\
\hline 4760 & $\mathrm{C}$ & \\
\hline 4761 & $\mathrm{C}$ & STORE TABLE DATA \\
\hline 4762 & $\mathrm{C}$ & \\
\hline 4763 & & DO $220 \quad I=1,5$ \\
\hline 4764 & & IF (ITBLU(I) .EQ. 99999) GO TO 210 \\
\hline 4765 & & IF (IUSER - ITBLU(I)) $220,200,220$ \\
\hline 4766 & 200 & $\operatorname{IUTBLD}(I, I M O N T H)=$ DELIVR $($ IUSER $)+.5$ \\
\hline 4767 & & $\operatorname{IUTBLP}(I$, IMONTH $)=$ PUMPAG (IUSER $)+.5$ \\
\hline 4768 & & $\operatorname{IUTBLT}(\mathrm{I}, \mathrm{IMONTH})=$ TOTAL +.5 \\
\hline 4769 & & GO TO 220 \\
\hline 4770 & 210 & IF (USRTYP(IUSER) .GT. 5) GO TO 220 \\
\hline 4771 & & $\operatorname{IUTBLD}(\mathrm{I}, \operatorname{IMONTH})=\operatorname{IUTBLD}(\mathrm{I}, \mathrm{IMONTH})+\mathrm{DELIVR}($ IUSER $) / 1000$. \\
\hline 4772 & & $\operatorname{IUTBLP}(I$, IMONTH $)=\operatorname{IUTBLP}(I$, IMONTH $)+\operatorname{PUMPAG}($ IUSER $) / 1000$. \\
\hline 4773 & & $\operatorname{IUTBLT}(\mathrm{I}, \mathrm{IMONTH})=\operatorname{IUTBLT}(\mathrm{I}, \mathrm{IMONTH})+\mathrm{TOTAL} / 1000$. \\
\hline 4774 & 220 & CONTINUE \\
\hline 4775 & $\mathrm{C}$ & \\
\hline 4776 & $\mathrm{C}$ & STORE STATISTICS DATA \\
\hline 4777 & $\mathrm{C}$ & \\
\hline 4778 & & DO $250 \mathrm{I}=1,10$ \\
\hline 4779 & & IF (ISSU(I) .EQ. 99999) G0 TO 240 \\
\hline 4780 & & IF (IUSER - ISSU(I)) $250,230,250$ \\
\hline 4781 & 230 & $\operatorname{UMENND}(I$, JMONTH$)=\operatorname{UMENND}(I$, JMONTH $)+\operatorname{NEED}($ IUSER $)$ \\
\hline 4782 & & $\operatorname{UMENDD}(\mathrm{I}, \mathrm{JMONTH})=\operatorname{UMENDD}(\mathrm{I}, \mathrm{JMONTH})+$ DELIVR (IUSER) \\
\hline 4783 & & UMENGW(I, JMONTH) $=$ UMENGW $(I$, JMONTH $)+$ PUMPAG(IUSER) \\
\hline 4784 & & $\operatorname{UMENRR}(I, J M O N T H)=\operatorname{UMENRR}(I, J M O N T H)+\operatorname{RESREL}($ IUSER) \\
\hline 4785 & & UMENTA $(I$, MMONTH $)=$ UMENTA $(I, J M O N T H)+$ TOTAL \\
\hline 4786 & & $\operatorname{UMENP}(I, J M O N T H)=\operatorname{UMENP}(I$, MMONTH$)+\operatorname{PRECIP}($ IUSER $)$ \\
\hline 4787 & & UMENWC $(I$, JMONTH $)=\operatorname{UMENWC~}(I$, JMONTH $)+$ CONSUM \\
\hline 4788 & & $\operatorname{UMENC}(\mathrm{I}, \mathrm{JMONTH})=\operatorname{UMENC}(\mathrm{I}, \mathrm{JMONTH})+\operatorname{APCONC}($ IUSER $)$ \\
\hline 4789 & & IF (IQW .GT. 0) UMENC (I, JMONTH) $=\operatorname{UMENC}(I$, JMONTH $)-A P C O N C$ (IUSER $)+$ \\
\hline 4790 & & 1 APCONC (IUSER)*(TOTAL+PRECIP(IUSER)) \\
\hline 4791 & & UMENCL $(\mathrm{I}, \mathrm{JMONTH})=\operatorname{UMENCL}(\mathrm{I}, \mathrm{JMONTH})+$ CANLEK (IUSER) \\
\hline 4792 & & $\operatorname{UMENTW}(I, J M O N T H)=\operatorname{UMENTW}(I, J M O N T H)+$ TAILWR (IUSER) \\
\hline 4793 & & UMENDP $(I$, MMNTH $)=$ UMENDP $(I, J M O N T H)+$ DPPERC (IUSER) \\
\hline 4794 & & GO TO 250 \\
\hline
\end{tabular}


Attachment 8--FORTRAN Source Code of Subroutines for Water-Supply Operations Simulation--Continued

4800

4801

4802

4803

4804

4805

4806

4807

4808

4809

4810

4811

4812

4813

4814

4815

4816

4817

4818

4819

4820

4821

4822

4823

4824

4825

4826

4827

4828

4829

4830

4831

4832

4833

4834

4835

4836

4837

4838

4839

4840

4841

4842

4843

4844

4845

4846

4847

240 IF (USRTYP(IUSER) .GT. 5) GO TO 250

$\operatorname{UMENND}(I, J M O N T H)=\operatorname{UMENND}(I, J M O N T H)+\operatorname{NEED}($ IUSER $) / 1000$.

$\operatorname{UMENDD}(\mathrm{I}, \mathrm{JMONTH})=\operatorname{UMENDD}(\mathrm{I}, \mathrm{JMONTH})+\mathrm{DELIVR}$ (IUSER) $/ 1000$.

$\operatorname{UMENGW}(\mathrm{I}, \mathrm{JMONTH})=\operatorname{UMENGW}(\mathrm{I}, \mathrm{JMONTH})+\operatorname{PUMPAG}($ IUSER $) / 1000$.

$\operatorname{UMENRR}(I, J M O N T H)=\operatorname{UMENRR}(I, J M O N T H)+\operatorname{RESREL}($ IUSER) $/ 1000$.

$\operatorname{UMENTA}(I, J M O N T H)=\operatorname{UMENTA}(I, J M O N T H)+$ TOTAL $/ 1000$.

$\operatorname{UMENP}(I, J M O N T H)=\operatorname{UMENP}(I, J M O N T H)+\operatorname{PRECIP}($ IUSER $) / 1000$.

$\operatorname{UMENWC}(I, J M O N T H)=\operatorname{UMENWC}(I, J M O N T H)+$ CONSUM $/ 1000$.

$\operatorname{UMENC}(I, J M O N T H)=\operatorname{UMENC}(I, J M O N T H)+\operatorname{APCONC}$ (IUSER)

IF (IQW .GT . 0) UMENC (I, JMONTH) $=\operatorname{UMENC~(I,~JMONTH)-APCONC~(IUSER)~+~}$

1 APCONC (IUSER)*(TOTAL+PRECIP(IUSER)) $/ 1000$.

UMENCL $(I$, JMONTH $)=\operatorname{UMENCL}(I$, JMONTH $)+$ CANLEK (IUSER) $/ 1000$.

$\operatorname{UMENTW}(\mathrm{I}, \mathrm{JMONTH})=\operatorname{UMENTW}(\mathrm{I}, \mathrm{JMONTH})+\mathrm{TAILWR}($ IUSER $) / 1000$.

$\operatorname{UMENDP}(I, J M O N T H)=\operatorname{UMENDP}(I, J M O N T H)+\operatorname{DPPERC}($ IUSER $) / 1000$.

250 CONTINUE

C

WRITE $(52,1050)$ IUSER, USERID(IUSER), USRNAM(IUSER), NEED (IUSER),

1 DELIVR (IUSER), PUMPAG (IUSER), RESREL (IUSER), IMPORT (IUSER), TOTAL,

2 PRECIP (IUSER), CONSUM, APCONC (IUSER), CANLEK (IUSER), TAILWR (IUSER),

3 DPPERC (IUSER)

1050 FORMAT (I5, I8, 2X,A8, 2F8.0,2F9.0,F10.0,F9.0, F8.0,F10.0,2F9.0, 2F8.0)

260 CONTINUE

C

IF ( (TTTL + TPRCP) .NE. 0.) AVGCON = AVGCON $/($ TTTL + TPRCP $)$

WRITE $(52,1060)$ TNEED, TDLVR, TPUMP, TRES, TIMPRT , TTTL, TPRCP, TCONSM, 1 AVGCON, TLEAK, TTAIL, TDP

1060 FORMAT $(/, 15 \mathrm{X}, 6$ HTOTALS ,F10.0,F8.0,2F9.0,F10.0,F9.0,F8.0,F10.0, $12 \mathrm{~F} 9.0,2 \mathrm{~F} 8.0$ )

C

C

C

IF ISKMU $=12$, PLOT ANNUAL WATER USE SCHEMATIC

IF (ISKMU - 12) $300,270,300$

270 IF (JMONTH - 12) 300,280,300

280 WRITE (IDUM, 1070) TITL, IYEAR

1070 FORMAT $(5 A 4,22 \mathrm{H}$ - WATER USE FOR YEAR , I3, 1H\$)

CALL SKMU(ITITLE)

C

DO 290 IUSER $=1$, NUSERS

$\mathrm{ADD}$ (IUSER) $=0$.

$A G W($ IUSER $)=0$.

$A R R($ IUSER) $=0$.

$A I M($ IUSER) $=0$.

$A P R($ IUSER $)=0$.

ANEED (IUSER) $=0$.

$290 \mathrm{ACN}$ (IUSER) $=0$.

C

C

C

SUMMARIZE RESERVOIR DATA

300 WRITE $(52,1080)$

1080 FORMAT $(/ / /, 33 \mathrm{H}$ RESERVOIR RESERVOIR RESERVOIR ,/,

$199 \mathrm{H}$ NUMBER ID NAME CONTENTS CONCENTRATION RE

2LEASES INFLOWS IMPORTS EVAPORATION )

DO 410 IRES $=1$, NRES 
Attachment 8--FORTRAN Source Code of Subroutines

for Water-Supply Operations Simulation--Continued

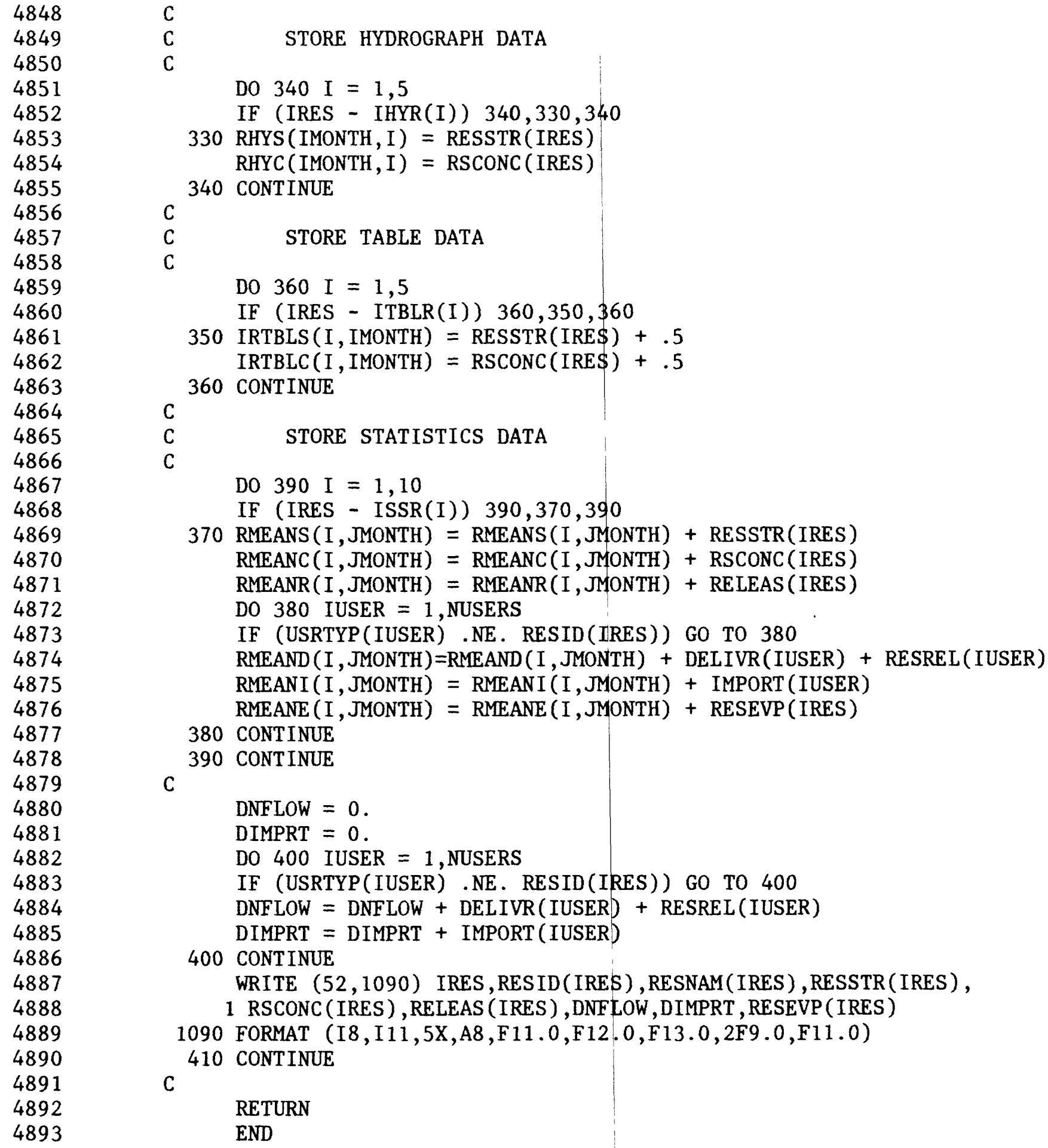


Attachment 8--FORTRAN Source Code of Subroutines

for Water-Supply Operations Simulation--Continued

\begin{tabular}{|c|c|c|}
\hline $\begin{array}{l}4894 \\
4895\end{array}$ & $\begin{array}{l}\text { CSUPPLY } \\
\text { C }\end{array}$ & \\
\hline 4896 & $\mathrm{C}$ & SUBROUTINE SUPPLY OF RIVER BASIN SIMULATION MODEL \\
\hline 4897 & $\mathrm{C}$ & WRITTEN BY ALAN BURNS, U.S. GEOLOGICAL SURVEY \\
\hline 4898 & $\mathrm{C}$ & WATER RESOURCES DIVISION, LAKEWOOD, CO \\
\hline 4899 & C & LATEST UPDATE $5 / 21 / 87$ \\
\hline 4900 & $\mathrm{C}$ & \\
\hline 4901 & & SUBROUTINE SUPPLY \\
\hline 4902 & & COMMON NMONTH, NINDV, IN, IOUT ; IPR INT, I GRAPH, IBATCH, IQW, IMONTH, \\
\hline 4903 & & $1 \quad$ JMONTH, IYEAR \\
\hline 4904 & & COMMON /DEMND/ NUSERS,USERID (100), XLOCU (100), YLOCU (100), \\
\hline 4905 & & DMDFAC $(100), \operatorname{USRTYP}(100)$, NUMUSR (100), NSOURC $(100)$ \\
\hline 4906 & & $\operatorname{RFTYPE}(100), \operatorname{RFVAL}(100), \operatorname{RFXLOC}(100), \operatorname{RFYLOC}(100)$ \\
\hline 4907 & & NODERF $(100), \operatorname{RFDIST}(100), \operatorname{ICODE}(100,15), \operatorname{IPRECP}(100)$, \\
\hline 4908 & & QUANTY $(100,15), \operatorname{SCXLOC}(100,15), \operatorname{SCYLOC}(100,15)$ \\
\hline 4909 & & NODESC $(100,15), \operatorname{WANT}(100), \operatorname{NEED}(100), \operatorname{USXPLT}(100)$, \\
\hline 4910 & & USYPLT $(100), \operatorname{ISIDRF}(100), \operatorname{IAKNT}(100), \operatorname{ITIMFC}(100,15)$, \\
\hline 4911 & & $\operatorname{TIMFAC}(100,12)$ \\
\hline 4912 & & INTEGER USERID,USRTYP,RFTYPE \\
\hline 4913 & & REAL NUMUSR, NEED \\
\hline 4914 & & COMMON /DEMND1/ USRNAM(100) \\
\hline 4915 & & DOUBLE PRECISION USRNAM \\
\hline 4916 & & COMMON /GW/ GWSTOR $(100,2), \operatorname{GWCONC}(100,2), \operatorname{RTRNFL}(100,2)$ \\
\hline 4917 & & COMMON /GWUSE/ MGW, GWUSER( 100$)$, GWSOUR( 100$), \operatorname{GWDIST}(100)$, GWPUMP (100) \\
\hline 4918 & & $1 \quad, \operatorname{ISDPMP}(100)$ \\
\hline 4919 & & INTEGER GWUSER, GWSOUR \\
\hline 4920 & & COMMON /HYDGRG/ IHYGW(5), GWHYS $(600,5), \operatorname{GWHYC}(600,5), \operatorname{GWHYRF}(600,5)$ \\
\hline 4921 & & COMMON /HYDGRR/ IHYR (5), RHYS $(600,5), \operatorname{RHYC}(600,5)$ \\
\hline 4922 & & COMMON /HYDGRU/ IHYU (5), UHYD $(600,5), \operatorname{UHYP}(600,5), \operatorname{UHYT}(600,5)$ \\
\hline 4923 & & COMMON /PHYSIO/ STCOEF, TRANS, PET (12), DRINK, SESNAL (12), RMILES (100), \\
\hline 4924 & & $1 \quad \operatorname{EVAP}(12), \operatorname{UNIT}(5,480)$, CNVLAT , CVLONG , SNLAT 1, SNLAT2, \\
\hline 4925 & & SNUOS 1 , SNUOS 2 , SNUOS 3 , PREFAC, SEEPFC, PRSPFC, TAILFC, \\
\hline 4926 & & $\operatorname{AGDMND}(12)$, CANLEN $(100)$, RAIN (50) \\
\hline 4927 & & COMMON /REACHS/ NNODE, NODE ID (100), NXTNOD $(100), \operatorname{XLOC}(100), \mathrm{YLOC}(100)$, \\
\hline 4928 & & PLTX $(100), \operatorname{PLTY}(100), \operatorname{NTRCPT}(100,12), \operatorname{SLOPE}(100,12)$, \\
\hline 4929 & & QWA $(100,12), Q W B(100,12), \operatorname{INDEPV}(100,12), Q(100)$ \\
\hline 4930 & & QW(100), CONCFC (100), NODEUS (100), QSUBA (100), \\
\hline 4931 & & QWSUBA $(100), \mathrm{DSA}(100), \mathrm{DSB}(100)$ \\
\hline 4932 & & REAL NTRCPT \\
\hline 4933 & & COMMON /RESUSE/ MRES, RESUSR(100), RESSOR (100), NUMRES (100), \\
\hline 4934 & & $1 \quad \operatorname{TRANSL}(100), \operatorname{RESRL}(100), \operatorname{ISBACT}(100), \operatorname{RESQNT}(100)$ \\
\hline 4935 & & INTEGER RESUSR, RESSOR \\
\hline 4936 & & COMMON /RESVOR/ NRES, RESSTR (25), RELEAS (25), NODRES (25), RESID (25), \\
\hline 4937 & & $1 \quad \operatorname{CAPMAX}(25), \operatorname{NACTIV}(25), \operatorname{AREAMX}(25), \operatorname{RSXLOC}(25)$ \\
\hline 4938 & & RSYLOC (25), RSCONC (25), RESEVP (25), RSXPLT (25), \\
\hline 4939 & & RSYPLT $(25), \operatorname{RESCUM}(25), \operatorname{SBSTOR}(25,5)$ \\
\hline 4940 & & INTEGER RESID \\
\hline 4941 & & REAL NACTIV \\
\hline 4942 & & COMMON /RES1/ RESNAM(25) \\
\hline 4943 & & DOUBLE PRECISION RESNAM \\
\hline 4944 & & COMMON /RIGHTS/ NRIGHT, OWNER (200) \\
\hline 4945 & & INTEGER OWNER \\
\hline 4946 & & COMMON /STATR/ ISSR (10), RMEANS $(10,12)$ \\
\hline
\end{tabular}


4947

4948

4949

4950

4951

4952

4953

4954

4955

4956

4957

4958

4959

4960

4961

4962

4963

4964

4965

4966

4967

4968

4969

4970

4971

4972

4973

4974

4975

4976

4977

4978

4979

4980

4981

4982

4983

4984

4985

4986

4987

4988

4989

4990

4991

4992

4993

4994

4995

4996

4997

4998

4999

5000

5001
$1 \quad \operatorname{RMEAND}(10,12), \operatorname{RMEANI}(10,12), \operatorname{RMEANE}(10,12)$

COMMON /TABLEG/ ITBLG(5), IGTBLS $(5,600), \operatorname{IGTBLC}(5,600), \operatorname{IGTBLR}(5,600)$

COMMON /TABLER/ ITBLR(5), IRTBLS $(5,600), \operatorname{IRTBLC}(5,600)$

COMMON /TABLEU/ ITBLU(5), IUTBLD $(5,600), \operatorname{IUTBLP}(5,600), \operatorname{IUTBLT}(5,600)$

COMMON /USTATS/ DELIVR(100), RESREL (100), IMPORT (100), PUMPAG (100),

1

2

REAL IMPORT $\operatorname{APCONC}(100), \operatorname{DIVRZN}(100), \operatorname{PUMPG}(100,2), \operatorname{CANLEK}(100)$, DPPERC (100), TAILWR (100), PRECIP (100)

$\mathrm{C}$

$\mathrm{C}$

DIMENSION QQ(100), QAVAIL (100), PICKUP(100), RESCON (25), WANNT (100)

INTEGER USRID

$\operatorname{INTEGER} \approx 2 \operatorname{USED}(100,15)$

C

C

INITIALIZE ACCOUNTING

C

DO 10 INODE $=1$, NNODE

PICKUP (INODE) $=Q($ INODE $)$

QAVAIL (INODE) $=$ Q(INODE)

$\mathrm{QQ}($ INODE $)=\mathrm{Q}($ INODE $)$

$\operatorname{DIVRZN~(INODE)~}=0$.

PUMPG(INODE, 1$)=0$.

10 PUMPG(INODE, 2) $=0$.

C

NNOD1 $=$ NNODE -1

DO 20 INODE $=1$, NNOD 1

IDOWN $=$ NXTNOD (INODE)

IF (IDOWN .LT. 0) GO TO 20

PICKUP (IDOWN) = PICKUP (IDOWN $)-Q($ INODE $)$

IF (PICKUP (IDOWN) .LT. 0.) PICKUP (IDOWN) $=0$.

C

20

CONTINUE

DO 40 IRES $=1$, NRES

RESCON (IRES) $=$ RESSTR (IRES) - RESEVP (IRES)

RELEAS (IRES) $=0$.

IF (IMONTH .EQ. 1) RESCUM(IRES) $=0$.

IF (JMONTH .EQ. 10) RESCUM(IRES) $=0$.

IF (RESSTR(IRES) .EQ. O.) GO TO 40

DO 30 IACCNT $=1,5$

30 SBSTOR (IRES, IACCNT) $=$ SBSTOR (IRES, IACCNT) *RESCON (IRES) $/$ RESSTR (IRES)

c

40 CONTINUE

DO 80 IUSER $=1$, NUSERS

DELIVR (IUSER) $=0$.

PUMPAG (IUSER) $=0$.

RESREL(IUSER) $=0$.

IMPORT (IUSER) $=0$.

WANNT (IUSER) = WANT (IUSER)

IF (USRTYP (IUSER) .EQ. 1 .AND. DMDFAC (IUSER) .IT . 0.)

1 WANNT (IUSER) $=\operatorname{PET}($ JMONTH $) *$ NUMUSR $($ IUSER $) *(-$ DMDFAC $($ IUSER $))$

DO 70 ISOURC $=1,15$

USED (IUSER, ISOURC) $=0$

IF (ICODE (IUSER, ISOURC) .NE. 3) GO TO 70

DO 60 JRES $=1$, MRES

IF (RESUSR(JRES) .NE. IUSER .OR. RESSOR(JRES) .NE. ISOURC) 
Attachment 8--FORTRAN Source Code of Subroutines

for Water-Supply Operations Simulation--Continued

5002

5003

5004

5005

5006

5007

5008

5009

5010

5011

5012

5013

5014

5015

5016

5017

5018

5019

5020

5021

5022

5023

5024

5025

5026

5027

5028

5029

5030

5031

5032

5033

5034

5035

5036

5037

5038

5039

5040

5041

5042

5043

5044

5045

5046

5047

5048

5049

5050

5051

5052

5053

1 GO TO 60

IF (QUANTY(IUSER, ISOURC) .GE. 0.) GO TO 50

IF (IMONTH .NE. 1 .AND. JMONTH .NE. 4) GO TO 60

IRES = NUMRES (JRES)

IACCNT = ISBACT (JRES)

RESQNT (JRES) = -QUANTY (IUSER, ISOURC) *SBSTOR (IRES, IACCNT)

GO TO 60

50 RESQNT (JRES) = QUANTY (IUSER, ISOURC)

60 CONTINUE

70 CONTINUE

80 CONTINUE

C

C

DO $90 \mathrm{JGW}=1, \mathrm{MGW}$

$\operatorname{GWPUMP}(J G W)=0$.

90 CONTINUE

C

100

DO 100 JRES $=1$, MRES

$\operatorname{RESRL}(J R E S)=0$.

C

$\mathrm{C}$

GO THROUGH THE DIRECT DIVERSION PRIORITY SYSTEM

DO 440 IRIGHT $=1$, NRIGHT

USRID = OWNER (IRIGHT)

DO 110 IUSER $=1$, NUSERS

IF (USRID .EQ. USERID(IUSER)) GO TO 120

110 CONTINUE

GO TO 440

120 IF (WANT(IUSER) .LE. 0.) GO TO 440

C

C

C

C

GO THROUGH THE LIST OF SOURCES FOR THIS USER UNTIL THE DIRECT DIVERSION IS FOUND

ISOURC $=0$

130 ISOURC $=$ ISOURC +1

IF (SOURC .GT. NSOURC(IUSER)) GO TO 440

IF (USED(IUSER, ISOURC) .NE. 0) GO TO 130

C

TYMFAC $=1$.

IF (ITIMFC (IUSER, ISOURC) .EQ. 0) GO TO 140

NTIMFC = ITIMFC (IUSER, ISOURC)

TYMFAC $=$ TIMFAC (NTIMFC, JMONTH $)$

C

140 CONTINUE

GO TO $(150,320,350,400,240,270)$, ICODE (IUSER, ISOURC)

C

C

C

DIRECT DIVERSION IN PRIORITY

150 INODE $=$ NODESC (IUSER, ISOURC)

INODUP $=$ NODEUS (INODE $)$

DIVERT = AMIN1 (WANT (IUSER), QUANTY (IUSER, ISOURC)*TYMFAC*60.3,

1 QAVAIL (INODUP) $\div 60.3$ ) 
Attachment 8--FORTRAN Source Code of Subroutines

for Water-Supply Operations Simulation--Continued

5054

5055

5056

5057

5058

5059

5060

5061

5062

5063

5064

5065

5066

5067

5068

5069

5070

5071

5072

5073

5074

5075

5076

5077

5078

5079

5080

5081

5082

5083

5084

5085

5086

5087

5088

5089

5090

5091

5092

5093

5094

5095

5096

5097

5098

5099

5100

5101

5102

5103

5104

5105

5106

5107
160 IF (DIVERT .LT. 0.05) DIVERT $=0$.

IF (DIVERT .EQ. 0.) GO TO 440

DELIVR (IUSER) = DELIVR (IUSER) + DIVERT

WANT (IUSER) = WANT (IUSER) - DIVERT

WANNT (IUSER) $=$ WANNT (IUSER) - DIVERT

USED (IUSER, ISOURC) $=1$

DIVERT $=$ DIVERT $/ 60.3$

DIVRZN (INODE) $=$ DIVRZN (INODE) + DIVERT

ADJUST $=0$.

JNODE = INODUP

170 IF (QQ(JNODE) .LE. 0.) GO TO 190

QAVAIL (JNODE) = QAVAIL (JNODE) - DIVERT - ADJUST

$Q Q(J N O D E)=Q Q(J N O D E)-D I V E R T$ - ADJUST

IF (QQ (JNODE) .GE. 0.) GO TO 180

ADJUST $=Q Q(J N O D E)+$ ADJUST

180 JNODE $=$ NXTNOD $($ JNODE $)$

IF (JNODE .GT. O) GO TO 170

NNODE $=$ NNODE +1

$190 \mathrm{QQ}($ JNODE $)=\mathrm{QQ}(\mathrm{JNODE})-\mathrm{DIVERT}$ - ADJUST

INODE = INODUP

200 ADJUST $=$ AMIN 1 (PICKUP $($ INODE $)$, DIVERT)

PICKUP (INODE) = PICKUP (INODE) - ADJUST

DIVERT = DIVERT - ADJUST

IF (DIVERT .LE. O.) GO TO 440

$210 \mathrm{JNODE}=0$

DO 220 JNODE $1=1$, NNOD 1

INODE $1=$ NXTNOD ( JNODE 1 )

IF (INODE 1 .NE. INODE) GO TO 220

IF (QAVAIL(JNODE 1) .LE. 0.) GQ TO 220

JNODE $=$ JNODE 1

220 CONTINUE

IF (JNODE .NE. 0) GO TO 230

INODE = NXTNOD (INODE)

GO TO 210

230 QAVAIL (JNODE) = QAVAIL (JNODE) - DIVERT

INODE $=$ JNODE

GO TO 200

$\mathrm{C}$

$\mathrm{C}$

C

240 INODE $=$ NODESC $($ IUSER, ISOURC $)$

INODUP $=$ NODEUS (INODE)

DO 250 IRES $=1$, NRES

IF (RESID(IRES) .EQ. USRTYP(IUSER)) GO TO 260

250 CONTINUE

260 IACCNT $=$ IAKNT $($ IUSER $)$

DIVERT = AMIN 1 ( CAPMAX (IRES) -RESCON (IRES) $) *$ TYMFAC,

1 QAVAIL (INODUP) *60.3, (NUMUSR (IUSER) -SBSTOR (IRES, IACCNT)) *TYMFAC,

2 QUANTY(IUSER, ISOURC)-RESCUM(IRES) )

IF (DIVERT .LT. 0.05) DIVERT $=0$.

RESCON (IRES) $=$ RESCON (IRES) + DIVERT

SBSTOR (IRES, IACCNT) = SBSTOR (IRES, IACCNT) + DIVERT

RESCUM (IRES) $=$ RESCUM (IRES) + DIVERT

GO TO 160 
Attachment 8--FORTRAN Source Code of Subroutines

for Water-Supply Operations Simulation--Continued

5108

5109

5110

5111

5112

5113

5114

5115

5116

5117

5118

5119

5120

5121

5122

5123

5124

5125

5126

5127

5128

5129

5130

5131

5132

5133

5134

5135

5136

5137

5138

5139

5140

5141

5142

5143

5144

5145

5146

5147

5148

5149

5150

5151
C

C DIRECT DIVERSION EXCHANGED TO STORAGE RIGHT

C

270 IF (TYMFAC .NE. 0.) GO TO 150

INODE = NODESC (IUSER, ISOURC)

INODUP $=$ NODEUS (INODE)

DO 280 JRES $=1$, NRES

IF (RESUSR(JRES).EQ. IUSER .AND. RESSOR(JRES).EQ. ISOURC) GO TO 290 280 CONTINUE

290 IRES $=$ NUMRES (JRES)

IACCNT = ISBACT (JRES)

INODRS $=$ NODRES (IRES)

INDRSU $=$ NODEUS $($ INODRS $)$

DIVERT = AMIN1 (WANT (IUSER), QUANTY (IUSER, ISOURC) $* 60.3$,

1 QAVAIL (INODUP) $* 60.3$, QAVAIL (INDRSU) $* 60.3$ )

DO 300 JUSER $=1$, NUSERS

IF (RESID (IRES) .EQ. USRTYP(JUSER) .AND. NSOURC(JUSER) .EQ. 0)

1 GO TO 310

300 CONTINUE

310 DIVERT = AMIN1 (DIVERT, CAPMAX (IRES)-RESCON (IRES),

1 NUMUSR (JUSER) -SBSTOR (IRES, IACCNT) )

IF (DIVERT .LT. 0.05) DIVERT $=0$.

IF (DIVERT .EQ. 0.) GO TO 440

RESCON(IRES) = RESCON(IRES) + DIVERT

SBSTOR (IRES, IACCNT) = SBSTOR (IRES , IACCNT) + DIVERT

WANT (IUSER) = WANT (IUSER) - DIVERT

WANNT (IUSER) = WANNT (IUSER) - DIVERT

USED (IUSER, ISOURC) $=1$

$\operatorname{DELIVR}($ JUSER $)=\operatorname{DELIVR}($ JUSER $)+$ DIVERT

DIVERT = DIVERT $/ 60.3$

DIVRZN (INODRS) $=$ DIVRZN (INODRS $)+$ DIVERT

ADJUST $=0$.

INODUP $=$ INDRSU

JNODE = INODUP

GO TO 170

C

C

C

$\mathrm{C}$

320 INODE $=$ NODESC (IUSER, ISOURC)

DO $330 \mathrm{JGW}=1, \mathrm{MGW}$

IF (GWUSER(JGW) .EQ. IUSER .AND. GWSOUR(JGW) .EQ. ISOURC)

1 GO TO 340

330 CONTINUE 
Attachment 8--FORTRAN Source Code of Subroutines

for Water-Supply Operations Simulation--Continued

5152

5153

5154

5155

5156

5157

5158

5159

5160

5161

5162

5163

5164

5165

5166

5167

5168

5169

5170

5171

5172

5173

5174

5175

5176

5177

5178

5179

5180

5181

5182

5183

5184

5185

5186

5187

5188

5189

5190

5191

5192

5193

5194

5195

5196

5197

5198

5199

5200

5201

5202

5203
C

340 ISIDE $=$ ISDPMP $(\mathrm{JGW})$

DIVERT = AMIN1 (WANNT $($ IUSER $)$, QUANTY $($ IUSER, ISOURC $) \div$ TYMFAC *60.3,

1 GWSTOR(INODE, ISIDE) )

IF (DIVERT .LT . 0.05) DIVERT $=0$.

IF (DIVERT .EQ. 0.) GO TO 130

WANT (IUSER) $=$ WANT (IUSER) - DIVERT

WANNT (IUSER) $=$ WANNT (IUSER) - DIVERT

CALL CMPUTR (-DIVERT, INODE, ISIDE, GWDIST (JGW))

PUMPAG (IUSER) = PUMPAG (IUSER) + DIVERT

PUMPG (INODE, ISIDE) = PUMPG (INODE, ISIDE) + DIVERT

GWPUMP $(J G W)=$ DIVERT

USED (IUSER, ISOURC) $=1$

GO TO 130

C

C

C

350 DO 390 JRES $=1$, MRES

IF (RESUSR(JRES) .NE. IUSER .OR. RESSOR (JRES) .NE. ISOURC)

1 GO TO 390

INODE $=$ NODESC (IUSER, ISOURC)

IRES = NUMRES (JRES)

IACCNT $=$ ISBACT $($ JRES $)$

RESWNT = WANNT (IUSER) $/$ TRANSL (JRES)

RELES = AMIN1 (RESWNT, RESQNT (JRES) *TYMFAC, SBSTOR (IRES , IACCNT) )

C

IF (USRTYP(IUSER) .LT. 5) GO TO 380

DO 360 KRES $=1$, NRES

IF (RESID(KRES) .EQ. USRTYP(IUSER)) GO TO 370

360 CONTINUE

370 KACCNT $=$ IAKNT $($ IUSER $)$

RELES = AMIN1 (RELES, (CAPMAX (KRES) -RESCON (KRES))*TYMFAC,

1 (NUMUSR (IUSER) -SBSTOR (KRES, KACCNT)) *TYMFAC)

RESCON(KRES) = RESCON(KRES) + RELES *TRANSL(JRES)

C

SBSTOR $($ KRES, KACCNT) $=$ SBSTOR (KRES, KACCNT) + RELES $* T R A N S L(J R E S)$

380 IF (RELES .LT. 0.05) RELES $=0$.

RELEAS (IRES) = RELEAS (IRES) + RELES

RESCON (IRES) $=$ RESCON(IRES) - RELES

SBSTOR (IRES, IACCNT) = SBSTOR (IRES, IACCNT) - RELES

$\operatorname{RESQNT}(J R E S)=\operatorname{RESQNT}(\mathrm{JRES})-$ RELES

DIVERT = RELES $*$ TRANSL $(J R E S)$

RESREL (IUSER) = RESREL (IUSER) + DIVERT

$\operatorname{RESRL}(J R E S)=$ DIVERT

WANT (IUSER) $=$ WANT (IUSER) - DIVERT

WANNT (IUSER $)=$ WANNT $($ IUSER $)-$ DIVERT

USED (IUSER, ISOURC) $=1$

DIVERT $=$ DIVERT $/ 60.3$

IF (INODE . LE. NNODE) DIVRZN (INODE) $=$ DIVRZN (INODE) + DIVERT

GO TO 130

390 CONTINUE

GO TO 130 
Attachment 8--FORTRAN Source Code of Subroutines

for Water-Supply Operations Simulation--Continued

5204

5205

5206

5207

5208

5209

5210

5211

5212

5213

5214

5215

5216

5217

5218

5219

5220

5221

5222

5223

5224

5225

5226

5227

5228

5229

5230

5231

5232

5233

5234

5235

5236

5237

5238

5239

5240

5241

5242

5243

5244

5245

5246
$\mathrm{C}$

$\mathrm{C}$

C

400 INODE $=$ NODESC $($ IUSER, ISOURC $)$

DIVERT $=$ AMIN 1 (QUANTY (IUSER, ISOURC) *TYMFAC , WANNT (IUSER),

1 QAVAIL (INODE) $\div 60.3$ )

IF (DIVERT .LT . 0.05) DIVERT $=0$.

IF (DIVERT .EQ. O.) GO TO 130

IF (USRTYP(IUSER) .LT. 5) GO TO 430

DO 410 IRES = 1 , NRES

IF (RESID(IRES) .EQ. USRTYP(IUSER)) GO TO 420

410 CONTINUE

420 IACCNT $=$ IAKNT (IUSER)

DIVERT = AMIN1 (DIVERT, $($ CAPMAX $($ IRES $)-$ RESCON $($ IRES $)) *$ TYMFAC,

1 (NUMUSR (IUSER) -SBSTOR (IRES, IACCNT)) $\div$ TYMFAC)

RESCON (IRES) = RESCON (IRES) + DIVERT

SBSTOR (IRES, IACCNT) = SBSTOR (IRES, IACCNT) + DIVERT

430 IF (DIVERT .LT . 0.05) DIVERT $=0$.

WANT (IUSER) $=$ WANT (IUSER) - DIVERT

WANNT (IUSER) = WANNT (IUSER) - DIVERT

$\operatorname{IMPORT}($ IUSER $)=$ IMPORT (IUSER) + DIVERT

USED (IUSER, ISOURC) $=1$

GO TO 130

C

C

440 CONTINUE

C

$\mathrm{C}$

DO 590 IUSER $=1$, NUSERS

IF (WANT(IUSER) .LE. O.) GO TO 590

ISOURC $=0$

450 ISOURC $=$ ISOURC +1

IF (ISOURC . GT. NSOURC (IUSER)) GO TO 590

IF (USED(IUSER, ISOURC) .NE. 0) GO TO 450

C

IF (WANT (IUSER) .LE. O.) GO TO 590

TYMFAC $=1$.

IF (ITIMFC (IUSER, ISOURC) .EQ. 0) GO TO 460

NTIMFC = ITIMFC (IUSER, ISOURC)

TYMFAC $=$ TIMFAC $($ NTIMFC, JMONTH $)$

460 CONTINUE

C

GO TO $(450,470,500,550,450,450)$, ICODE (IUSER, ISOURC) 
Attachment 8--FORTRAN Source Code of Subroutines for Water-Supply Operations Simulation--Continued

$\mathrm{C}$

C

C

C

470 INODE $=$ NODESC (IUSER, ISOURC)

DO $480 \mathrm{JGW}=1, \mathrm{MGW}$

IF (GWUSER(JGW) .EQ. IUSER .AND. GWSOUR(JGW) .EQ. ISOURC) 1 GO TO 490

480 CONTINUE

C

490 ISIDE $=\operatorname{ISDPMP}(\mathrm{JGW})$

DIVERT = AMIN1 (WANNT (IUSER), QUANTY (IUSER, ISOURC) $*$ TYMFAC $* 60.3$,

1 GWSTOR(INODE, ISIDE) )

IF (DIVERT . LT . 0.05) DIVERT $=0$.

IF (DIVERT .EQ. 0.) GO TO 450

WANT (IUSER) $=$ WANT (IUSER) - DIVERT

WANNT (IUSER) $=$ WANNT (IUSER) - DIVERT

CALL CMPUTR (-DIVERT, INODE, ISIDE, GWDIST (JGW))

PUMPG (INODE, ISIDE) = PUMPG (INODE, ISIDE) + DIVERT

PUMPAG (IUSER) = PUMPAG(IUSER) + DIVERT

GWPUMP $(J G W)=$ DIVERT

USED (IUSER, ISOURC) $=1$

GO TO 450 
Attachment 8--FORTRAN Source Code of Subroutines

for Water-Supply Operations Simulation--Continued

5270

5271

5272

5273

5274

5275

5276

5277

5278

5279

5280

5281

5282

5283

5284

5285

5286

5287

5288

5289

5290

5291

5292

5293

5294

5295

5296

5297

5298

5299

5300

5301

5302

5303

5304

5305

5306

5307
C

C

C

500 DO 540 JRES $=1$, MRES

IF (RESUSR(JRES) .NE. IUSER .OR. RESSOR(JRES) .NE. ISOURC)

1 GO TO 540

INODE $=$ NODESC (IUSER, ISOURC)

IRES = NUMRES (JRES)

IACCNT = ISBACT $(J R E S)$

RESWNT = WANNT (IUSER) $/$ TRANSL (JRES)

RELES = AMIN1 (RESWNT, RESQNT (JRES)*TYMFAC, SBSTOR (IRES , IACCNT) )

C

IF (USRTYP(IUSER) .LT. 5) GO TO 530

DO 510 KRES $=1$, NRES

IF (RESID(KRES) .EQ. USRTYP(IUSER)) GO TO 520

510 CONTINUE

520 KACCNT $=$ IAKNT (IUSER)

RELES = AMIN1 (RELES,$($ CAPMAX (KRES) -RESCON (KRES) $) \div$ TYMFAC,

1 (NUMUSR (IUSER) -SBSTOR (KRES, KACCNT)) *TYMFAC)

RESCON(KRES) = RESCON(KRES) + RELES*TRANSL (JRES)

SBSTOR (KRES , KACCNT) $=$ SBSTOR(KRES, KACCNT) + RELES $*$ TRANSL (JRES)

C

530 IF (RELES .LT. 0.05) RELES $=0$.

RELEAS (IRES) $=$ RELEAS (IRES) + RELES

RESCON(IRES) = RESCON(IRES) - RELES

SBSTOR (IRES, IACCNT) = SBSTOR (IRES, IACCNT) - RELES

RESQNT (JRES) = RESQNT (JRES) - RELES

DIVERT = RELES*TRANSL (JRES)

RESREL (IUSER) = RESREL (IUSER) + DIVERT

RESRL (JRES) = DIVERT

WANT (IUSER) $=$ WANT (IUSER) - DIVERT

WANNT (IUSER) $=$ WANNT (IUSER) - DIVERT

USED (IUSER, ISOURC) $=1$

DIVERT $=$ DIVERT $/ 60.3$

IF (INODE .LE. NNODE) DIVRZN(INODE) = DIVRZN (INODE) + DIVERT

GO TO 450

540 CONTINUE

GO TO 450 
Attachment 8--FORTRAN Source Code of Subroutines

for Water-Supply Operations Simulation--Continued

5308

5309

5310

5311

5312

5313

5314

5315

5316

5317

5318

5319

5320

5321

5322

5323

5324

5325

5326

5327

5328

5329

5330

5331

5332

5333

5334
C

C IMPORTED WATER

C

550 INODE $=$ NODESC $($ IUSER, ISOURC $)$

DIVERT=AMIN 1 (QUANTY (IUSER, ISOURC) *TYMFAC , WANNT (IUSER),

1 QAVAIL (INODE) *60.3)

IF (DIVERT .LT . 0.05) DIVERT $=0$.

IF (DIVERT .EQ. O.) GO TO 450

IF (USRTYP(IUSER) .LT. 5) GO TO 580

DO 560 IRES $=1$, NRES

IF (RESID(IRES) .EQ. USRTYP(IUSER)) GO TO 570

560 CONTINUE

570 IACCNT $=$ IAKNT (IUSER)

DIVERT = AMIN1 $($ DIVERT,$($ CAPMAX (IRES $)-$ RESCON $($ IRES $)) *$ TYMFAC,

1 (NUMUSR (IUSER) -SBSTOR (IRES, IACCNT))*TYMFAC)

RESCON (IRES) = RESCON (IRES) + DIVERT

SBSTOR (IRES, IACCNT) = SBSTOR (IRES, IACCNT) + DIVERT

580 WANT $($ IUSER $)=$ WANT (IUSER $)$ - DIVERT

WANNT (IUSER) $=$ WANNT (IUSER) - DIVERT

IMPORT (IUSER) = IMPORT (IUSER) + DIVERT

USED (IUSER, ISOURC) $=1$

GO TO 450

C

590 CONTINUE

C

RETURN

END 
Attachment 8--FORTRAN Source Code of Subroutines for Water-Supply Operations Simulation--Continued

5335 CUNTRSP

5336 C

5337 C

$5338 \quad \mathrm{C}$

5339 C

$5340 \quad \mathrm{C}$

$5341 \quad \mathrm{C}$

SUBROUTINE UNIT RESPONSE OF RIVER BASIN SIMULATION MODEL

WRITTEN BY ALAN BURNS, U.S. GEOLOGICAL SURVEY

WATER RESOURCES DIVISION, LAKEWOOD, CO

LATEST UPDATE $9 / 10 / 86$

5342

5343

5344

5345

5346

5347

5348

5349

5350

5351

5352

5353

5354

5355

5356

5357

5358

5359

5360

5361

5362

5363

5364

5365

5366

5367

5368

5369

5370

5371

5372

5373

5374

5375

5376

5377

5378

5379

5380

5381

5382

5383

5384

C

C

C

SUBROUTINE UNTRSP

COMMON NMONTH, NINDV, IN , IOUT, IPRINT, I GRAPH, IBATCH, IQW, IMONTH,

1 JMONTH, IYEAR

COMMON /PHYSIO/ STCOEF, TRANS, PET (12),DRINK, SESNAL (12), RMILES (100),

1

2

3

COMMON /REACHS/

1

2

3

4

REAL NTRCPT EVAP (12), UNIT $(5,480)$, CNVLAT , CVLONG , SNLAT 1, SNLAT2, SNUOS 1 , SNUOS 2 , SNUOS 3 , PREFAC, SEEPFC, PRSPFC, TAILFC, AGDMND (12), CANLEN ( 100), RAIN (50)

COMMON /RESPON/ FRSPNZ $(100,480,2), \operatorname{TLWATR}(100,2), \operatorname{TWCONC}(100,2)$

DIMENSION DIST (5)

DOUBLE PRECISION ZUNIT, A, A2 , B , B2 , C , C2, ZI , TIME, AERFC , SDF

DATA DIST /1000.,2000.,5000.,10000.,20000./

DO $30 \mathrm{~J}=1,5$

$\mathrm{SDF}=\operatorname{DIST}(\mathrm{J}) \div \operatorname{DIST}(\mathrm{J}) \div \mathrm{STCOEF} / \mathrm{TRANS}$

$\mathrm{A}=0.5 \mathrm{DO} * \mathrm{DSQRT}(\mathrm{SDF} / 30.4 \mathrm{DO})$

$\mathrm{A} 2=\mathrm{A} * \mathrm{~A}$

ZUNIT $=(2.0 \mathrm{D} 0 * \mathrm{~A} 2+1.0 \mathrm{DO}) * \mathrm{AERFC}(\mathrm{A})-1.12838 \mathrm{D} 0 * \mathrm{~A} * \mathrm{DEXP}(-\mathrm{A} 2)$

$\operatorname{UNIT}(J, 1)=$ ZUNIT

$\operatorname{IF}(\operatorname{UNIT}(\mathrm{J}, 1)$.LT. 0.) $\operatorname{UNIT}(\mathrm{J}, 1)=0$.

DO $10 \mathrm{I}=2,480$

$\mathrm{ZI}=\mathrm{I}$

TIME $=30.4 \div \mathrm{ZI}$

$\mathrm{A}=0.5 \mathrm{D} 0 * \mathrm{DSQRT}(\mathrm{SDF} / \mathrm{TIME})$

$A 2=A * A$

$\mathrm{B}=0.5 \mathrm{D} 0 * \mathrm{DSQRT}(\mathrm{SDF} /(\mathrm{TIME}-30.4 \mathrm{DO}))$

$\mathrm{B} 2=\mathrm{B} * \mathrm{~B}$

$C=0.0 \mathrm{DO}$

IF (I .NE. 2) $\mathrm{C}=0.5 \mathrm{D} 0 * \mathrm{DSQRT}(\mathrm{SDF} /(\mathrm{TIME}-60.8 \mathrm{D0}))$

$\mathrm{C} 2=\mathrm{C} * \mathrm{C}$

ZUNIT $=((2.0 \mathrm{D} 0 * \mathrm{~A} 2+1.0 \mathrm{D} 0) * \mathrm{AERFC}(\mathrm{A})-1.12838 \mathrm{D} 0 * \mathrm{~A} * \mathrm{DEXP}(-\mathrm{A} 2)) * \mathrm{ZI}-$

$1((2.0 \mathrm{DO} * \mathrm{~B} 2+1.0 \mathrm{DO}) * \mathrm{AERFC}(\mathrm{B})-1.12838 \mathrm{D} 0 * \mathrm{~B} * \mathrm{DEXP}(-\mathrm{B} 2)) *(\mathrm{ZI}-1.0 \mathrm{DO}) * 2.0 \mathrm{DD} 0$

$2+((2.0 \mathrm{DO} * \mathrm{C} 2+1.0 \mathrm{DO}) * \mathrm{AERFC}(\mathrm{C})-1.12838 \mathrm{D} 0 * \mathrm{C} * \mathrm{DEXP}(-\mathrm{C} 2)) *(\mathrm{ZI}-2.0 \mathrm{DO})$

$\operatorname{UNIT}(\mathrm{J}, \mathrm{I})=$ ZUNIT

10 CONTINUE 
Attachment 8--FORTRAN Source Code of Subroutines for Water-Supply Operations Simulation--Continued

5385

5386

5387

5388

5389

5390

5391

5392

5393
30 CONTINUE

$\mathrm{C}$

DO 40 INODE $=1$, NNODE

DO 40 ITIME $=1,480$

DO 40 ISIDE $=1,2$

40 FRSPNZ (INODE, ITIME, ISIDE) $=0$.

RETURN

END 
Attachment 8--FORTRAN Source Code of Subroutines

for Water-Supply Operations Simulation--Continued

5394

5395

5396

5397

5398

5399

5400

5401

5402

5403

5404

5405

5406

5407

5408

5409

5410

5411

5412

5413

5414
C

C

C

DOUBLE PRECISION A1, A2, A3, A4, A5, A6, Z

IF (Z.LT.0.) WRITE $(1,1000)$

IF (Z.LT.O.) STOP

AERFC $=0.0 \mathrm{DO}$

IF (X.GE.4.) GO TO 10

$\mathrm{Al}=0.0705230784 \mathrm{DO}$

$\mathrm{A} 2=0.0422820123 \mathrm{D} 0$

$A 3=0.0092705272 \mathrm{DO}$

$\mathrm{A} 4=0.0001520143 \mathrm{D} 0$

$\mathrm{A} 5=0.0002765672 \mathrm{DO}$

$\mathrm{A} 6=0.0000430638 \mathrm{D} 0$

AERFC $=1.0 \mathrm{DO} /(1.0 \mathrm{D} 0+Z *(\mathrm{~A} 1+Z *(A 2+Z *(A 3+Z *(A 4+Z *(A 5+Z * A 6)))))) \div 16$

10 CONTINUE

RETURN

C

1000 FORMAT (53H NEGATIVE ARGUEMENT FOR COMPLEMENTERY ERROR FUNCTION ) 